

Surrogate Outcome Markers and Cerebral Haemodynamics in Carotid Interventions

Marjolijn L. Rots 


\section{Surrogate Outcome Markers and Cerebral Haemodynamics in Carotid Interventions}

PhD thesis, Utrecht University, the Netherlands

Financial support by the Dutch Heart Foundation for the publication of this thesis is gratefully acknowledged.

Publication of this thesis was additionally supported by Chirurgisch Fonds UMC Utrecht, stichting TWIN, Angiocare, Terumo Aortic Nederland and Chipsoft.

ISBN 978-94-6375-721-8

Cover Manon de Beer

Lay-out Thomas van der Vlis, persoonlijkproefschrift.nl

Printing Ridderprint BV I www.ridderprint.nl

(C) Marjolijn Lette Rots, Utrecht 2019

All rights reserved. No part of this thesis may be reproduced or transmitted in any form or by any means without prior written permission form the author. The copyright of the papers that have been published or have been accepted for publication has been transferred to the respective journals. 


\title{
Surrogate Outcome Markers and Cerebral Haemodynamics in Carotid Interventions
}

\author{
Surrogaat uitkomstmaten en cerebrale hemodynamica \\ bij behandeling van de halsslagader \\ (met een samenvatting in het Nederlands)
}

\section{Proefschrift}

ter verkrijging van de graad van doctor aan de Universiteit Utrecht op gezag van de rector magnificus, prof. dr. H.R.B.M. Kummeling, ingevolge het besluit van het college voor promoties in het openbaar te verdedigen op vrijdag 9 oktober 2020 des middags te 12.45 uur

door

\section{Marjolijn Lette Rots}

geboren op 17 september 1988

te Naarden 
Promotoren: Prof. dr. G.J. de Borst

Prof. dr. J. Hendrikse

Copromotor: Dr. J.J.M. Zwanenburg 


\section{Table of Contents}

Chapter 1 General introduction and thesis outline

\section{Part I: Surrogate markers for outcome in patients with carotid artery disease}

Chapter 2 Surrogate markers and reporting standards for outcome after carotid intervention

Eur J Vasc Endovasc Surg 2019

Commentary on "Overview of Primary and Secondary Analyses From 20 Randomised Controlled Trials Comparing Carotid Artery Stenting with Carotid Endarterectomy"

Eur J Vasc Endovasc Surg 2019

Chapter 3 Predictors of new ischaemic brain lesions on diffusion weighted imaging after carotid stenting and endarterectomy: a systematic review Eur J Vasc Endovasc Surg 2019

Chapter 4 Magnetic resonance imaging identified brain ischaemia in symptomatic patients undergoing carotid endarterectomy is related to histologically apparent intraplaque haemorrhage Eur J Vasc Endovasc Surg 2019

Chapter 5 Cerebral small vessel disease in standard preoperative imaging reports is independently associated with increased risk of cardiovascular death following carotid endarterectomy Eur J Vasc Endovasc Surg 2020

Chapter 6 Gadolinium-enhancement of the aneurysm wall in extracranial carotid artery aneurysms Am J Neuroradiol 2020

Part II: Changes in cerebral haemodynamics in patients undergoing carotid intervention

Chapter 7 Intraoperative hypotension is a risk factor for postoperative silent brain ischaemia in patients with preoperative hypertension undergoing carotid endarterectomy

Eur J Vasc Endovasc Surg 2020

Chapter 8 Effect of bilateral carotid occlusion on cerebral haemodynamics and perivascular innervation: An experimental rat model J Comp Neurol 2019

Chapter 9 The effect of carotid revascularization on cerebral arterial function as measured by 7 -Tesla MRI

In preparation

\section{Part III: Discussion and summary}

Chapter 10 Summarizing discussion, future perspectives and conclusion 155

$\begin{array}{lll}\text { Chapter } 11 \text { Summary in Dutch I Nederlandse samenvatting } 167 & 16\end{array}$

$\begin{array}{ll}\text { Appendices Review committee } & 174\end{array}$

Authors and affiliations $\quad 176$

List of publications $\quad 178$

Acknowledgements I Dankwoord 180

About the author 182 


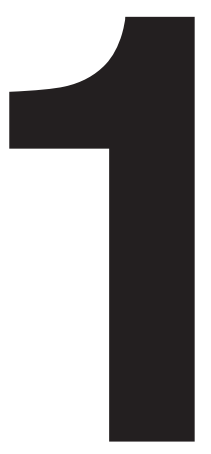

General introduction and thesis outline 


\section{General Introduction}

This thesis describes various aspects to improve care for patients with carotid artery disease. It mainly focusses on how to select the right treatment regime for each patient with the lowest chance of periprocedural complications and simultaneously preserving a high chance of treatment benefit. In the following introduction, background information pertinent to this topic will be discussed and a brief outline of the thesis will be provided.

\section{Stroke}

The brain is one of the best perfused organs of our body, as it is constantly involved in intricate tasks and bodily functions. Complex mechanisms interact to maintain a continuous blood supply under varying (internal and external) conditions and as such maintain a constant delivery of oxygen and nutrients to the vulnerable brain tissue. When blood supply to a region of the brain is interrupted, the brain is deprived from these essentials, followed by damaged brain tissue, eventually causing a loss of neurological function or even death. When loss of neurological function lasts more than 24 hours this is defined as' "Stroke", while a transient ischaemic attack (TIA) by definition lasts less than 24 hours. Stroke is one of the leading causes of disability worldwide, with large proportions of patients remaining dependent on some form of care for daily activities ${ }^{2}$. Stroke can be divided into two main aetiological categories. Ischaemic stroke is defined as a hampering of arterial blood supply due to thrombosis, embolism or hypoperfusion and is responsible for approximately $80 \%$ of all strokes ${ }^{3}$. Haemorrhagic stroke causes damage to the brain by direct compression from blood to surrounding brain tissue. The latter is outside the scope of this thesis and hereinafter mentioning of 'stroke' will refer to ischaemic stroke.

\section{Carotid artery disease}

The brain is supplied of oxygen-rich blood by four major arteries: two internal carotid arteries and two vertebral arteries. Ten to fifteen percent of all ischaemic strokes are caused by carotid artery disease ${ }^{4}$. Generally, carotid artery disease is of atherosclerotic origin in which an atherosclerotic plaque narrows the lumen of the carotid artery (stenosis). Carotid artery disease may result in ischaemic stroke by two types of mechanisms. In the majority of cases, an instable 'plaque' in the vessel wall ruptures, resulting in emboli to the brain that cause occlusion of more distally located intracranial vessels ${ }^{5}$. Notably, although much less common, such emboli may also occur in case of a widening of the arterial wall, known as an extracranial carotid aneurysm (ECAA). Another type of stroke can occur as a result of hampered passage of blood to the brain due to carotid stenosis or occlusion (complete blockage of the carotid artery). Under normal conditions, collateral circulation should be able to compensate for such a drop in unilateral blood supply. Nonetheless, carotid artery disease patients are often exposed to multilevel arterial disease and/or have a non-competent cerebral circulation, which may jointly lead to cerebral hypoperfusion. This hypoperfusion may result in cerebral ischaemia in the so-called watershed areas of the brain, which are borderzone-areas of the major cerebral arteries, susceptible to (temporary) changes in perfusion pressure ${ }^{5}$. Coexistence of 
hypoperfusion and arterial embolism in patients with carotid artery disease may also play a role in the aetiology of stroke ${ }^{6}$.

\section{Carotid intervention}

Carotid interventions are indicated in previously symptomatic patients and are known as tertiary prevention strategies. Carotid Endarterectomy (CEA) and Carotid Artery Stenting (CAS) have both been proven to reduce the long-term risk of stroke in patients with symptomatic carotid artery stenosis ${ }^{7}$. Both procedures aim to eliminate the atherosclerotic plaque of the affected carotid artery in order to reduce risk of future stroke. Asymptomatic patients (no TIA/stroke in the last 6 months) with carotid artery disease tend to benefit less from such revascularisation procedures and are generally treated with medical therapy only (secondary prevention) $)^{8}$. In symptomatic patients, CEA is the first choice of treatment with lower risk of perioperative cerebrovascular events compared to stenting procedures ${ }^{9}$.

Optimal treatment strategy, either carotid intervention or best medical treatment (BMT), in asymptomatic or low-risk symptomatic patients is still under debate ${ }^{8,10}$. Revascularisation procedures may not only reduce risk of thrombo-embolic events but, especially in case of multi-vessel disease, may also effectuate a change in cerebral haemodynamics by improving blood flow through the ipsilateral carotid artery. Yet, not much is known about how changes in cerebral perfusion influence blood flow in deep 'perforating' cerebral arteries. Chronic hypoperfusion can lead to cognitive decline and are associated with increased risk of stroke ${ }^{11,12}$. Therefore, knowledge on possible improvements of perforating artery functionality as a result of a revascularisation procedure may contribute to the process of decision making on optimal treatment strategy in asymptomatic carotid disease patients. Improvements in selection of certain patient subgroups and advances in diagnostic and therapeutic techniques may ultimately cause a shift in choice of treatment. Yet, with periprocedural stroke-rate gradually decreasing over the last decades, huge amounts of patients are required to demonstrate a significant outcome effect of new treatment algorithms. As such, there is a strong need for a redefinition of study endpoints and a warrant for surrogate markers for ischaemic stroke. Diffusion weighted imaging (DWI) lesions have emerged as a promising surrogate endpoint of periprocedural stroke. These DWI-lesions can be identified within minutes of a hypoxic episode and will be visible for several days. Most DWI-lesions do not cause clinical neurological symptoms and do not result in permanent ischaemic changes on follow-up imaging ${ }^{13}$. DWIlesions qualify as a strong surrogate measure, as they meet three conditions. First they have a biological plausible relation with stroke ${ }^{14}$. Second, they are associated with increased risk of future postprocedural stroke or $\mathrm{TI}^{15}$ and finally treatment effects on DWI-lesions correlates to treatment effects on stroke ${ }^{16}$. For example, it was demonstrated that new postprocedural ischaemic brain lesions on magnetic resonance diffusion-weighted imaging (MR-DWI) are much more common after CAS (50\%) compared to CEA (18\%) ${ }^{17}$. Accordingly, assessing presence of new postoperative DWI-lesions offers an excellent surrogate endpoint for studies evaluating treatment modifications.

Since the appearance of hyperintensities on MR-DWI is time-limited, this modality is not suitable to investigate brain damage related to the natural course of carotid artery disease or 
long-term outcome of carotid interventions. Instead, silent brain infarcts (SBIs) or white matter lesions (WMLs) could be used for this purpose. SBIs, and especially cortical infarcts are caused by emboli from proximal sources like the heart and for example carotid arteries. New silent and clinical brain infarcts in the ipsilateral hemisphere during follow-up could be used as a surrogate endpoint to evaluate the beneficial effects of carotid intervention compared to BMT in newly defined high risk-subgroups based on atherosclerotic plaque imaging. WMLs have been used widely as a marker for small vessel-disease and are closely associated with aging and risk factors for atherosclerosis such as hypertension ${ }^{18}$. WMLs may result from embolic events from unstable carotid plaques but may as well be an expression of generalized atherosclerotic disease ${ }^{19}$. All three surrogate markers have been associated with increased risk of future cerebrovascular events in patients with carotid artery disease ${ }^{15,20,21}$. Both SBIs and WMLs have been associated with increased risk of cognitive decline ${ }^{22,23}$.

\section{Thesis Outline}

In this thesis we investigate surrogate markers for outcome after carotid interventions and how such a procedure interacts with cerebral haemodynamics.

Part I focusses on surrogate markers for outcome after carotid interventions. In chapter 2 we provide an overview and comparison of available imaging surrogate outcome markers for carotid interventions and we discuss the need for clarity on reporting standards of these markers in scientific papers. In chapter $\mathbf{3}$ we describe a systematic review on patient characteristics that are predictive for development of silent ischaemic lesions on MR-DWI after CEA or CAS. Although CEA has proven to be more beneficial in terms of periprocedural outcome compared to CAS in the majority of patients, this difference between the two types of procedures is less evident for specific patient subgroups such as those of $<70$ years of age ${ }^{19}$. Identification of certain patient characteristics that contribute to development of ischaemic lesions can help in selecting an optimal individualized treatment strategy. In chapter 4 we investigate one of these patient-related features, carotid intraplaque haemorrhage (IPH), and its relation to presence of preoperative silent ischaemic lesions on MR-DWI. We demonstrate that presence of IPH increases the chance of such lesions on preoperative MRI and therefore advocate that screening for IPH at hospital presentation can help in prioritizing patients that may benefit more from early revascularisation. In chapter 5 we discuss the presence of preoperative WMLs as a potential predictor for perioperative and long-term outcome after CEA. We found that WMLs, as mentioned in standard clinical imaging reports, does not predict short- or long-term stroke risk, but is associated with long-term cardiovascular death. These findings can be helpful as part of a more complex clinical decision-making algorithm for selection of the most optimal treatment strategy based on several imaging characteristics. In chapter 6 we focus on extracranial carotid artery aneurysms (ECAAs) and methods to detect aneurysm instability and its relation to cerebral complications such as SBIs and WMLs. We conducted an explorative study investigating the feasibility of ECAA wall contrast enhancement on MRI and its relation to aneurysm growth and cerebral complications. 
Part II focusses on cerebral haemodynamics and its relation to carotid artery disease and the effect of treatment by revascularisation procedures. In the first chapter of this part (chapter 7) we investigate the relation between intraoperative blood pressure and development of new perioperative DWI lesions. We demonstrate that more knowledge on optimal pre- intra- and postoperative blood pressure management is warranted to be able to optimally protect patients undergoing a revascularisation procedure from cerebral hypoperfusion. Chapter 8 describes an experimental animal study in which we simulated the effect of chronic hypoperfusion on cerebral haemodynamics and perivascular nerve density. In doing so we try to seek a better understanding of the cerebral autoregulation and how this is affected by carotid artery disease. In chapter 9 we show how innovative imaging techniques can contribute to knowledge on the effect of carotid intervention on the arterial function of the brain. These novel techniques may eventually contribute to future decision making for choice of treatment in patients with carotid artery disease. 


\section{References}

1. Truelsen T, Begg S, Mathers C. The global burden of cerebrovascular disease. WHO.

2. Truelsen T, Piechowski-Jóźwiak B, Bonita R, Mathers C, Bogousslavsky J, Boysen G. Stroke incidence and prevalence in Europe: A review of available data. Eur J Neurol. 2006;13(6):581-98.

3. Grysiewicz RA, Thomas K, Pandey DK. Epidemiology of Ischemic and Hemorrhagic Stroke: Incidence, Prevalence, Mortality, and Risk Factors. Neurol Clin. 2008;26(4):871-95.

4. Inzitari D, Eliasziw M, Gates P, Sharpe BL, Chan RKT, Meldrum HE, et al. The Causes and Risk of Stroke in Patients with Asymptomatic Internal-Carotid-Artery Stenosis. N Engl J Med. 2002;342(23):1693-701.

5. Bonati LH, Brown MM. Carotid artery disease. In: Grotta J, Broderick J, Lo E, Sacco R, Albers G, Kasner S, et al., editors. Stroke. sixth. Elsevier; 2016.

6. Caplan LR, Hennerici M. Impaired clearance of emboli (washout) is an important link between hypoperfusion, embolism, and ischemic stroke. Arch Neurol. 1998;55(11):1475-82.

7. Group ECSTC. Randomised trial of endarterectomy for recently symptomatic carotid stenosis: final results of the MRC European Carotid Surgery Trial (ECST). 1998;351(9113):1379-87.

8. Gaba K, Ringleb PA, Halliday A. Asymptomatic Carotid Stenosis: Intervention or Best Medical Therapy? Curr Neurol Neurosci Rep. 2018;18(11):1-9.

9. Ederle J, Davagnanam I, van der Worp HB, Venables GS, Lyrer PA, Featherstone RL, et al. Effect of whitematter lesions on the risk of periprocedural stroke after carotid artery stenting versus endarterectomy in the International Carotid Stenting Study (ICSS): A prespecified analysis of data from a randomised trial. Lancet Neurol. 2013;12(9):866-72.

10. Mortimer R, Nachiappan S, Howlett DC. The role of imaging in screening special feaTure: Where are we now? Br J Radiol. 2018;91(1090).

11. Duncombe J, Kitamura A, Hase Y, Ihara M, Kalaria R, Horsburgh K. Chronic cerebral hypoperfusion: a key mechanism leading to vascular cognitive impairment and dementia (VCID). Closing the translational gap between rodent models and human VCID. Clin Sci. 2017;131(19):2451-68.

12. Klijn CJM, Kappelle LJ, Tulleken CAF, van Gijn JM. Symptomatic Carotid Artery Occlusion: A Reappraisal of Hemodynamic Factors. Stroke. 1997;28(10):2084-2093.

13. Hauth EAM, Jansen C, Drescher R, Schwartz M, Forsting M, Jaeger HJ, et al. MR and clinical follow-up of diffusion-weighted cerebral lesions after carotid artery stenting. Am J Neuroradiol. 2005 Oct;26(9):233641.

14. Lansberg MG, Norbash AM, Marks MP, Tong DC, Moseley ME, Albers GW. Advantages of adding diffusionweighted magnetic resonance imaging to conventional magnetic resonance imaging for evaluating acute stroke. Arch Neurol. 2000;57(9):1311-6.

15. Gensicke H, Van Der Worp HB, Nederkoorn PJ, Macdonald S, Gaines PA, Van Der Lugt A, et al. Ischemic brain lesions after carotid artery stenting increase future cerebrovascular risk. J Am Coll Cardiol. 2015;65(6):521-9.

16. Traenka C, Engelter ST, Brown MM, Dobson J, Frost C, Bonati LH. Silent brain infarcts on diffusionweighted imaging after carotid revascularisation: A surrogate outcome measure for procedural stroke? A systematic review and meta-analysis. Eur Stroke J. 2019;4(2):127-43.

17. Bonati LH, Jongen LM, Haller S, Flach HZ, Dobson J, Nederkoorn PJ, et al. New ischaemic brain lesions on MRI after stenting or endarterectomy for symptomatic carotid stenosis: a substudy of the ICSS. Lancet Neurol. 2010;9(4):353-62.

18. Inzitari D. Leukoaraiosis an independent risk factor for stroke? Stroke. 2003;34(8):2067-71.

19. Naylor AR, Ricco JB, de Borst GJ, Debus S, de Haro J, Halliday A, et al. Management of Atherosclerotic Carotid and Vertebral Artery Disease: 2017 Clinical Practice Guidelines of the ESVS. Eur J Vasc Endovasc Surg. 2018;55(1):3-81.

20. Streifler JY, den Hartog AG, Pan S, Pan H, Bulbulia R, Thomas DJ, et al. Ten-year risk of stroke in patients with previous cerebral infarction and the impact of carotid surgery in the Asymptomatic Carotid Surgery Trial. Int J Stroke. 2016;11(9):1020-7.

21. Streifler JY, Eliasziw M, Benavente OR, Alamowitch S, Fox AJ, Hachinski VC, et al. Prognostic importance of leukoaraiosis in patients with symptomatic internal carotid artery stenosis. Stroke. 2002;33(6):1651-5.

22. Vermeer SE, Longstreth WT, Koudstaal PJ. Silent brain infarcts: a systematic review. Lancet Neurol. 2007;6(7):611-9.

23. Van Dijk EJ, Prins ND, Vrooman HA, Hofman A, Koudstaal PJ, Breteler MMB. Progression of cerebral small vessel disease in relation to risk factors and cognitive consequences: Rotterdam scan study. Stroke. 2008;39(10):2712-9. 
General introduction and thesis outline 


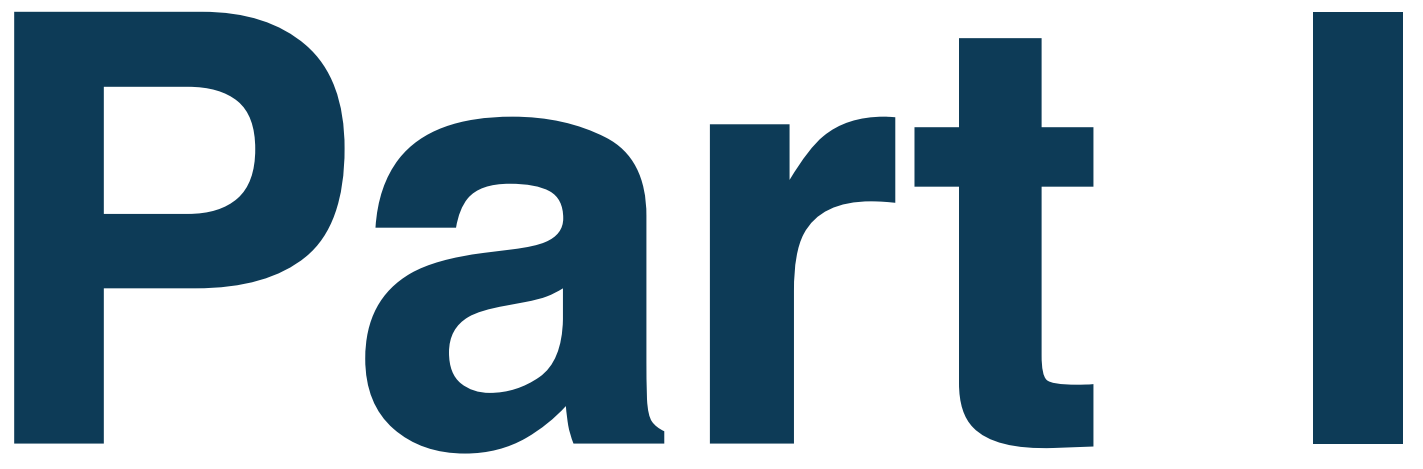

Surrogate markers for outcome in patients with carotid artery disease 


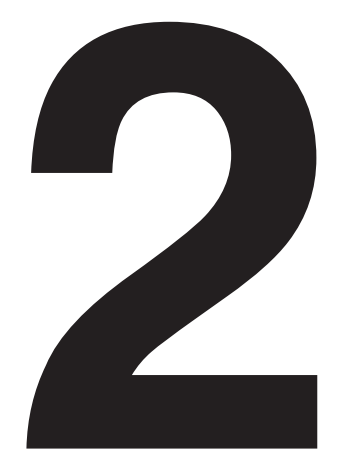

\section{Surrogate Markers and Reporting Standards for Outcome after Carotid Intervention}

\section{Commentary on "Overview of Primary and Secondary Analyses From 20 Randomised Controlled Trials} Comparing Carotid Artery Stenting with Carotid Endarterectomy" 


\section{Surrogate Markers and Reporting Standards for Outcome after Carotid Intervention}

Recently, focus on timing of intervention as well as improvements in medical therapy have changed the clinical treatment algorithm of carotid revascularization. The debate is still alive when it comes to the optimal treatment strategy including early $(<14 d)$ versus expedited $(<48 \mathrm{~h})$ revascularization, the role of plaque vulnerability in decision-making, and alternative (endovascular) revascularization approaches. In weighing the relative contribution and relevance of these strategies on outcome it is of utmost importance to apply universally accepted and clear endpoints. Since the classic clinical endpoint "procedural stroke and death" and "recurrent stroke" now occurs at low rates it would require huge amounts of patients to demonstrate a significant outcome effect of new diagnostic and treatment algorithms. As such there is a strong need for a redefinition of study endpoints and a warrant for surrogate markers for ischaemic stroke, both in the semi-acute and late phase.

It is important to stipulate that a distinction should be made between surrogate endpoints for periprocedural stroke versus surrogate markers for long-term outcome after revascularization or best medical treatment (BMT). Diffusion weighted imaging (DWI) lesions have emerged as a promising surrogate endpoint of periprocedural cerebral ischaemia. The main advantage of DWI is that ischaemic areas can be identified within minutes of a hypoxic episode and will be visible for several days. Most DWI-lesions are small, do not cause clinical neurological symptoms and do not result in permanent ischaemic changes on follow-up imaging 1 . DWIlesions are related to the procedure and far more prevalent than postprocedural strokes ${ }^{2}$. The MRI-substudy of the International Carotid Stenting Study demonstrated that presence of any new (ipsi- or contralateral) postprocedural DWI-lesion was considerably higher after carotid stenting $(50 \%)$ compared to endarterectomy $(17 \%)^{2}$. DWI-lesions qualify as a strong surrogate measure as they meet three conditions: 1) a biological plausible relationship with stroke $^{3}$ 2) an association with increased risk of postprocedural stroke/TIA ${ }^{4}$ and 3 ) the treatment effect on DWI-lesions correlates to that on stroke ${ }^{5}$. According to these three qualifications, DWI-lesions quantified as number and/or volume are an excellent surrogate endpoint for studies evaluating treatment modifications. A 2019 meta-analysis established that in case of an underlying $3 \%$ absolute difference in procedural stroke risk among revascularization techniques, a 90\%-sample size reduction could be achieved when using DWI-lesions instead of classic endpoints ${ }^{5}$. Of notice, little is known about the optimal timing of the postprocedural scan as both ongoing haemodynamic disturbance and thrombo-embolic events days after the procedure are common. Also, MR-field strength may influence the number of DWI-lesions ${ }^{2}$, and thus timing and image acquisition protocols should be harmonized in future research. Complete report of DWI data in randomized control trials of carotid interventions is required to use DWI as valid surrogate endpoint.

Since the appearance of hyperintensities on MR-DWI is time-limited, this modality is not suitable to investigate brain damage related to the natural course of atherosclerotic carotid artery disease. Instead, silent brain infarcts (SBIs) defined as cerebral infarcts on MRI (T2 or FLAIR) or CT without corresponding clinical symptoms could be used for this purpose. 
Sensitivity for detection of silent infarcts is higher for MRI compared to $\mathrm{CT}^{6}$ and reported prevalence of ipsilateral lesions is as high as $38 \%$ in patients with asymptomatic carotid stenosis $>50 \%$. Silent brain infarct, and especially cortical (micro-)infarcts are caused by emboli from proximal sources like the heart and atherosclerotic lesions in the aortic-arch, origin of supra-aortic arteries, carotid bifurcation and intracranial arteries. New silent and clinical brain infarcts in the ipsilateral hemisphere during follow-up could be used as a surrogate endpoint to evaluate the beneficial effects of carotid intervention compared to BMT in newly defined high risk-subgroups based on atherosclerotic plaque imaging.

A third possibility for determining long term outcome in carotid interventions is assessment of white matter-lesions (WMLs). WMLs have been used widely as a marker for small vesseldisease and are closely associated with aging and risk factors for atherosclerosis ${ }^{8}$, with hypertension being an important independent predictor for WML ${ }^{9}$. WMLs may result from embolic events from unstable carotid plaques but may as well be an expression of generalized atherosclerotic disease and until now evidence for causality between plaque instability and WML is fragile ${ }^{10}$. All three surrogate markers have been associated with increased risk of future cerebrovascular events in carotid artery disease patients specifically $y^{4,7,11}$. Both SBIs and WMLs have been associated with increased risk of cognitive decline ${ }^{6,12}$.

Concluding, there is a need for establishment of well-defined surrogate endpoints for stroke in patients with carotid artery disease. Diffusion weighted imaging lesions, silent brain infarcts and white matter lesions are promising and complementary surrogate markers. Standardization of imaging protocols and reporting of outcome is required. We recommend to differentiate and report both "any" versus "ipsilateral" new lesion. DWIlesions are most suitable for assessment of periprocedural outcome whereas both silent brain infarcts and white matter lesions can be used for long-term outcome. In our view, pre- and postprocedural MR brain has to be implemented in standard care to encourage and facilitate employment of these surrogate markers in research and clinical practice when treating patients with carotid artery disease. 


\section{References}

1. Hauth EAM, Jansen C, Drescher R, Schwartz M, Forsting M, Jaeger HJ, et al. MR and clinical follow-up of diffusion-weighted cerebral lesions after carotid artery stenting. Am J Neuroradiol. 2005 Oct;26(9):233641.

2. Bonati LH, Jongen LM, Haller S, Flach HZ, Dobson J, Nederkoorn PJ, et al. New ischaemic brain lesions on MRI after stenting or endarterectomy for symptomatic carotid stenosis: a substudy of the ICSS. Lancet Neurol. 2010;9(4):353-62.

3. Lansberg MG, Norbash AM, Marks MP, Tong DC, Moseley ME, Albers GW. Advantages of adding diffusionweighted magnetic resonance imaging to conventional magnetic resonance imaging for evaluating acute stroke. Arch Neurol. 2000;57(9):1311-6.

4. Gensicke H, Van Der Worp HB, Nederkoorn PJ, Macdonald S, Gaines PA, Van Der Lugt A, et al. Ischemic brain lesions after carotid artery stenting increase future cerebrovascular risk. J Am Coll Cardiol. 2015;65(6):521-9.

5. Traenka C, Engelter ST, Brown MM, Dobson J, Frost C, Bonati LH. Silent brain infarcts on diffusionweighted imaging after carotid revascularisation: A surrogate outcome measure for procedural stroke? A systematic review and meta-analysis. Eur Stroke J. 2019;

6. Vermeer SE, Longstreth WT, Koudstaal PJ. Silent brain infarcts: a systematic review. Lancet Neurol. 2007;6(7):611-9.

7. Streifler JY, den Hartog AG, Pan S, Pan H, Bulbulia R, Thomas DJ, et al. Ten-year risk of stroke in patients with previous cerebral infarction and the impact of carotid surgery in the Asymptomatic Carotid Surgery Trial. Int J Stroke. 2016;11(9):1020-7.

8. Inzitari D. Leukoaraiosis an independent risk factor for stroke? Stroke. 2003;34(8):2067-71.

9. Muscari A, Faccioli L, Ghinelli M, Napoli C, Pirazzoli E, Puddu GM, et al. Hypertension and Other Determinants of White Matter Lesions in Stroke Patients. J Clin Hypertens. 2016;18(9):907-12.

10. Naylor AR, Ricco JB, de Borst GJ, Debus S, de Haro J, Halliday A, et al. Management of Atherosclerotic Carotid and Vertebral Artery Disease: 2017 Clinical Practice Guidelines of the ESVS. Eur J Vasc Endovasc Surg. 2018;55(1):3-81.

11. Streifler JY, Eliasziw M, Benavente OR, Alamowitch S, Fox AJ, Hachinski VC, et al. Prognostic importance of leukoaraiosis in patients with symptomatic internal carotid artery stenosis. Stroke. 2002;33(6):1651-5.

12. Van Dijk EJ, Prins ND, Vrooman HA, Hofman A, Koudstaal PJ, Breteler MMB. Progression of cerebral small vessel disease in relation to risk factors and cognitive consequences: Rotterdam scan study. Stroke. 2008;39(10):2712-9. 
Surrogate outcome markers and reporting standards 


\section{Commentary on "Overview of Primary and Secondary Analyses From 20 Randomised Controlled Trials Comparing Carotid Artery Stenting with Carotid Endarterectomy"}

Since classical endpoints such as periprocedural stroke and death during carotid interventions now occur at low rates, secondary outcome measures are being used progressively. Batchelder et al. performed a recent analysis of 20 randomized controlled trials investigating carotid endarterectomy (CEA) versus stenting (CAS) ${ }^{1}$. As a secondary outcome measure, they report more new white matter lesions (WMLs) after CAS compared to CEA. However, the term WML is generally reserved for lesions of the periventricular areas or deep white matter, which develop over time as a result of cerebral small vessel disease. New ischaemic lesions that appear on post-procedural imaging and result directly from thrombo-embolisms or haemodynamic events are generally not restricted to the white matter and will mostly occur in the cortical or subcortical areas².

Usage of the term WML in the periprocedural setting can be confusing and should be avoided. Occurrence of periprocedural lesions marked by a focal hyperintense area on diffusion weighted imaging (DWI) should be referred to as DWI-lesions. Irreversible ischaemic lesions that were not accompanied by any symptoms are better known as silent brain infarcts (SBIs). The term WML should be reserved for lesions actually restricted to the white matter which can be used for baseline imaging or as a surrogate marker for long-term outcome, although evidence of a direct causality between carotid artery disease and WMLs is fragile. Consistent reporting and terminology of these surrogate endpoints is critical, especially since a shift towards using these imaging markers as a primary outcome is imminent ${ }^{3}$. 


\section{References}

1. Batchelder AJ, Saratzis A, Ross Naylor A. Editor's Choice - Overview of Primary and Secondary Analyses From 20 Randomised Controlled Trials Comparing Carotid Artery Stenting With Carotid Endarterectomy. Eur J Vasc Endovasc Surg. 2019;58(4):479-93.

2. Gunduz Y, Akdemir R, Ayhan LT, Keser N. Can Doppler flow parameters of carotid stenosis predict the occurrence of new ischemic brain lesions detected by diffusion-weighted MR imaging after filter-protected internal carotid artery stenting? AJNR Am J Neuroradiol. 2014 Apr;35(4):760-5.

3. Rots ML, van der Lugt A, de Borst GJ. Surrogate Markers and Reporting Standards for Outcome After Carotid Intervention. Eur J Vasc Endovasc Surg. 2019;58(6):794-5. 


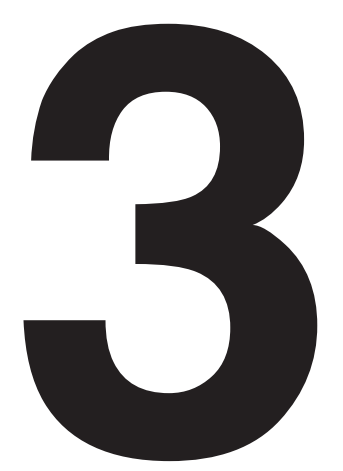

\section{Predictors of new ischaemic brain lesions on diffusion weighted imaging after carotid stenting and endarterectomy: a systematic review}

ML Rots, AJA Meershoek, LH Bonati, HM den Ruijter, GJ de Borst Eur J Vasc Endovasc Surg 2019;58(2):163-164 


\section{Abstract}

OBJECTIVES Periprocedural ischaemic brain lesions on diffusion weighted imaging (DWI) after carotid endarterectomy (CEA) and carotid artery stenting (CAS) have been related to a higher chance of recurrent cerebrovascular events. This systematic review provides an overview of patient characteristics associated with increased risk of new DWI lesions.

METHODS MEDLINE, EMBASE and Cochrane library databases were systematically searched (update November 2018) for studies reporting on post-procedural DWI lesions after CEA or CAS. Data derived from both procedures were analyzed separately. Studies reporting on predictive features that were present prior to intervention were assigned to ten categories: age, gender, cardiovascular risk factors, symptomatology, plaque vulnerability, atherosclerotic burden, cerebrovascular haemodynamics, carotid/arch-anatomy, inflammatory markers and markers of coagulation. A semi-quantitative analysis was performed by plotting studies finding an association between the investigated features and DWI lesions against those not finding an association.

RESULTS 46 studies (5018 patients) were included; 10 reported only on CEA, 33 on CAS and 3 on both interventions. $68 \%$ of 1873 CEA-patients and $55.9 \%$ of 3145 CAS-patients were symptomatic. Weighted prevalence of DWI lesions was $18.1 \%(95 \% \mathrm{Cl}: 14.0 \%-22.7 \%)$ in CEA-patients compared to $40.5 \%$ (95\% Cl: $35.4-45.7 \%)$ in CAS-patients. Studies reporting on CEA-patients predominantly found an increased risk in symptomatic patients (2/7 studies, including 848/1661 patients), those with impaired haemodynamics (5/5 studies), and increased inflammatory markers (2/3 studies). Studies reporting on CAS-patients often found a positive association with age (10/26 studies), high plaque vulnerability (25/34 studies) or complex carotid/arch anatomy (3/5 studies).

CONCLUSIONS For patients undergoing CEA, symptomatic status, impeded cerebral haemodynamics and increased inflammatory markers are associated with increased susceptibility for perioperative DWI lesions. In CAS-patients, higher age, plaque vulnerability and complex carotid/aortic arch-anatomy were identified as risk factors. These clinical predictors may assist with decision making on patient selection for medical treatment, CEA or CAS. 


\section{Introduction}

The occurrence of periprocedural ischaemic brain lesions on magnetic resonance imaging (MRI) after treatment of the carotid artery has been an increasing topic of interest ${ }^{1-3}$. A considerably high incidence of these so called diffusion weighted imaging (DWI) lesions are reported after both carotid artery stenting (CAS; 37-50\%) and carotid endarterectomy (CEA; $10-17 \%)^{1-3}$. DWI-lesions are associated with a higher risk of recurrent cerebrovascular events $^{4,5}$. In the MRI substudy of the ICSS (international Carotid Stenting Study) it was demonstrated that recurrent stroke or transient ischaemic attack (TIA) was more likely to occur in DWI-positive patients compared to DWI-negative patients (hazard ratio 2.85) ${ }^{4}$. DWI-positive patients are also more likely to develop definite brain infarction on follow-up MRI compared to DWI-negative patients ${ }^{5}$. Furthermore, presence of silent ischaemic lesions may lead to early onset cognitive decline and dementia ${ }^{6}$. These results suggest that periprocedural DWI lesions may be used as a surrogate and clinically relevant marker for cerebral ischaemia. DWI lesions on MRI reflect intracellular movement of water resulting in cytotoxic oedema, which are considered reminiscent of early pathological changes as a result of acute ischaemia ${ }^{7}$. Different theories regarding the aetiology of these lesions exist. During and after carotid artery stenting or endarterectomy, dislodgement of thrombotic material or atherosclerotic debris of vulnerable plaques can lead to cerebral embolization ${ }^{3}$. A second hypothesis is that haemodynamic and embolic mechanisms interact in development of new (silent) ischaemic lesions as a result of "impaired clearance of emboli".

Over the last few years several studies have been performed investigating occurrence of new DWI lesions in relation to procedural features, as well as patient characteristics. Besides optimizing interventional techniques and improving periprocedural antiplatelet therapies, identification of patients at risk for periprocedural DWI lesions can be helpful in determining the most optimal treatment strategy, ranging from CEA and CAS to best medical treatment (BMT). Novel insights supporting clinicians in decision making based on individual patient characteristics are needed. The aim of this study was to provide an overview of predictive features, based on patient characteristics, for the development of new DWI lesions due to treatment of the carotid artery. 


\section{Materials and Methods}

This systematic review was performed according to the Preferred Reporting Items for Systematic Reviews and Meta-Analyses (PRISMA) guidelines ${ }^{9}$.

\section{Search strategy}

A systematic search was performed in MEDLINE, EMBASE and Cochrane databases in February 2018, updated in November 2018. The Medical Subject Headings (MeSH) terms "diffusion magnetic resonance imaging", "endarterectomy" and "stents" were combined using various synonyms for DWI lesions and carotid artery stenting and endarterectomy. The full search strategy can be found in data supplement (I).

Original studies that reported on patients undergoing CEA or CAS and performed both a preand post-procedural MR-DWI scan were included. Exclusion criteria were case reports, case series reporting on $<10$ patients, animal studies and reviews. Investigated parameters had to be based on preoperative data, studies that only involved intra- or postoperative measurements were excluded. Articles in English, Dutch, German and French were considered.

Duplicates were removed and studies published in languages other than abovementioned were excluded. Two authors (MLR, AJAM) independently assessed full text eligibility based on title and abstract screening. Discordant judgments were addressed by consulting a third author (GJB). The reference list of all included papers was searched manually to identify missed but potentially relevant studies.

\section{Data processing \& assessment of methodological quality}

Data derived from CEA and CAS were analysed separately to account for inherit differences in type of patients selected for each procedure as well as the procedure itself. If quantitative or separated CEA/CAS data were not provided, corresponding authors were contacted. In case of no response the article was excluded. Newcastle-Ottawa Scale (NOS) was used to assess quality of cohort studies based on selection and comparability of study groups and ascertainment of the outcome of interest (average quality was defined as 6-7 points out of 9, good quality was defined as 8-9 point out of nine). In case of RCT's, Cochrane Collaboration's risk of bias tool was used for assessment of random sequence generation, allocation concealment, selective reporting, blinding and missing data ${ }^{10,11}$. Two authors (MLR, AJAM) independently assessed methodological quality and carried out data extraction from the individual papers.

\section{Statistical analysis}

All reported predictive parameters were collected and subsequently assigned to different categories (see table 1). Categories were as follows: Age, gender, cardiovascular risk factors, symptomatology, (ipsilateral) plaque burden or vulnerability, atherosclerotic burden, impaired cerebrovascular haemodynamics, difficult arch/carotid-anatomy, increased inflammatory markers and increased markers of coagulation. When a category was reported on in $<3$ articles, this category was excluded from further analysis. A semi-quantitative analysis was 
performed for each of the abovementioned categories, comparing features significantly associated with an increased risk of post-procedural DWI lesions to those for which no significant association was found. The results with total number of investigated patients were depicted in radar graphs.

Prevalence of DWI was estimated using a random effects model with double arcsine transformation ${ }^{12}$. Weighted means with $95 \%$ confidence interval $(\mathrm{Cl})$ of the proportion of patients with new post-procedural DWI lesions were calculated for CEA and CAS patients, subgroup analyses were performed for different MR-field strengths. Symptomatic and asymptomatic patients were analyzed together. Funnel plots were used to assess publication bias. MetaXL 5.3 (Epigear International Pty Lts) was used for statistical analyses.

Table 1 List of the predictive categories with the assigned parameters as reported by the included studies

\begin{tabular}{|c|c|}
\hline Category & Reported preprocedural predictive parameters \\
\hline Age & Age \\
\hline Gender & Gender \\
\hline $\begin{array}{l}\text { Cardiovascular } \\
\text { risk factors }\end{array}$ & $\begin{array}{l}\text { Hypertension; DM; hypercholesterolemia; HDL cholesterol, LDL cholesterol; currently } \\
\text { smoking; history of smoking; high BMI }\end{array}$ \\
\hline Symptomatology & $\begin{array}{l}\text { Symptomatic status (ischemic stroke; TIA or monocular vision loss in ipsilateral } \\
\text { carotid territory }<6 \text { months); preoperative stroke }<14 \text { days }\end{array}$ \\
\hline $\begin{array}{l}\text { Plaque burden/ } \\
\text { vulnerability }\end{array}$ & $\begin{array}{l}\text { High degree of stenosis; longer lesion; large plaque volume; jellyfish sign; plaque } \\
\text { ulceration; plaque calcification (proximal/distal); intraplaque haemorrhage; atheromic } \\
\text { plaque; fibrolipid plaque, large necrotic core; high intima media thickness; high- } \\
\text { intensity signal plaque; high signal intensity (ratio); high plaque muscle intensity ratio; } \\
\text { plaque echolucency; high extensive remodeling ratio; enhancement vaso vasorum; } \\
\text { eccentric lesion; plaque surface irregularity; floating plaque }\end{array}$ \\
\hline $\begin{array}{l}\text { Atherosclerotic } \\
\text { burden }\end{array}$ & Contralateral carotid lesion; CAD; PAOD; aortic plaques \\
\hline $\begin{array}{l}\text { Cerebrovascular } \\
\text { haemodynamics }\end{array}$ & $\begin{array}{l}\text { Impeded cerebrovascular reactivity to acetazolamide; high CBRBP/CBF asymmetry; } \\
\text { haemodynamic tandem intracranial lesion, reduced MCA signal intensity; high CBL / } \\
\text { MCA asymmetry; collateral flow; increased ICA PSV; increased ICA EDV }\end{array}$ \\
\hline $\begin{array}{l}\text { Arch/carotid } \\
\text { anatomy }\end{array}$ & $\begin{array}{l}\text { Aortic arch type II /III; CCA or ICA tortuosity (index); arch elongation; large ICA angle; } \\
\text { high CCA/ICA angle ratio; high expert score for anatomic suitability }\end{array}$ \\
\hline $\begin{array}{l}\text { Inflammatory } \\
\text { markers }\end{array}$ & Increased CRP; increased hsCRP; increased TNF-a \\
\hline $\begin{array}{l}\text { Markers of } \\
\text { coagulation }\end{array}$ & High fibrinogen; high platelet count \\
\hline
\end{tabular}

$\mathrm{DM}$ = diabetes mellitus; $\mathrm{BMI}=$ body mass index; $\mathrm{CAD}=$ coronary artery disease; $\mathrm{PAOD}=$ peripheral arterial occlusive disease;

$\mathrm{MCA}=$ middle cerebral artery $; \mathrm{CBL}=$ cerebellar hemispheric $; \mathrm{CBRBP}=$ central benzodiazepine receptor binding potential;

$\mathrm{CBF}=$ cerebral blood flow; ICA = internal carotid artery; PSV = peak systolic velocity; EDV = end diastolic velocity; $\mathrm{CRP}=\mathrm{C}$-reactive protein; hsCRP $=$ high-sensitive $=\mathrm{C}$-reactive protein. Plaque burden/vulnerability refers to the ipsilateral carotid artery. 


\section{Results}

\section{Study selection}

The search yielded 850 articles. After removal of duplicates, 695 articles were left of which 46 studies reporting on predictive features for development of DWI met the inclusion criteria (figure 1). An overview of the included studies is provided in table 2, full listing of studies including references can be found in the data supplement (II). Two substudies of randomized trials were included, other studies were prospective (29) and retrospective (15) cohort studies. The methodological quality of the included studies was high since all but one cohort study reached a NOS score of 7 or higher out of 9 and risk of bias was low for the two RCT substudies (table 2).

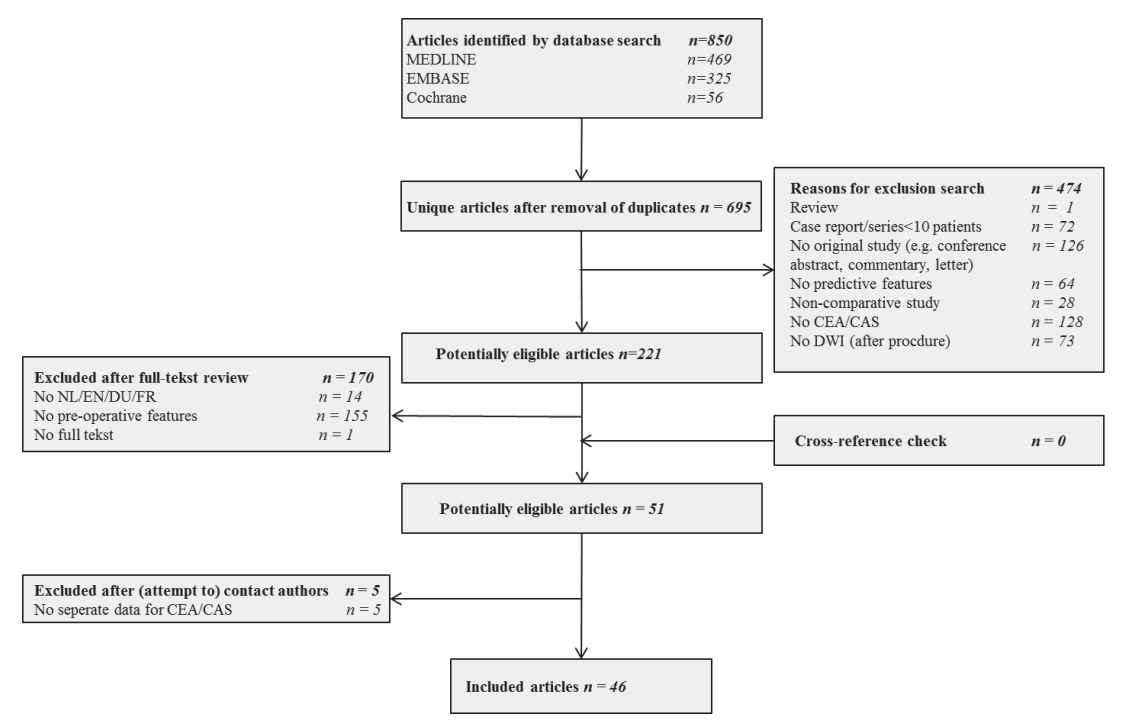

Figure 1 Flowchart showing selection of articles for review

\section{Study sample}

We included a total of 5018 unique patients from 46 studies of which 10 studies reported only on CEA-patients, 33 studies only on patient that had CAS and 3 studies reporting on both patient categories. For both CEA and CAS patients the same ten investigated features were described. A total of 1873 CEA-patients from 13 studies (68\% symptomatic) and 3145 CAS-patients from 36 studies (55.9\% symptomatic) were included. A weighted mean of $18.1 \%$ (95\% Cl: $14.0 \%-22.7 \%)$ new post-procedural DWI lesions in CEA-patients was significantly lower compared to $40.5 \%$ (95\% Cl: $35.4-45.7 \%$ in CAS-patients. There was no evidence of publication bias based on funnel plots.

Most studies ( $n=28$ ) used solely 1.5T MRI, some used 3T or a combination of $1.5 \mathrm{~T}$ and 3T ( $n=10$ and $n=2$ respectively), while six studies did not report the used field strength. Pooled prevalence of new DWI lesions after CEA in studies using 1.5T was 15.3\% (95\% Cl: $10.2-$ $21.2 \%$ ) compared to $14.1 \%$ (95\% Cl: $9.09-20.0 \%$ ) with $3 \mathrm{~T} \mathrm{MRI}$ and $30.3 \%$ (95\% Cl: 21.8 - 39.5\%) in combined studies. Reported pooled prevalence of new DWI lesions after CAS was $38.0 \%$ (95\% Cl: $32.4-43.7 \%)$ in studies using $1.5 \mathrm{~T} \mathrm{MRI}$, compared to $38.7 \%(95 \% \mathrm{Cl}$ : $27.8-50.3 \%)$ with 3 T and $48.1 \%(95 \% \mathrm{Cl}: 27.0-69.5 \%)$ in combined studies. 
Predictors of ischaemic brain lesions after carotid revascularization

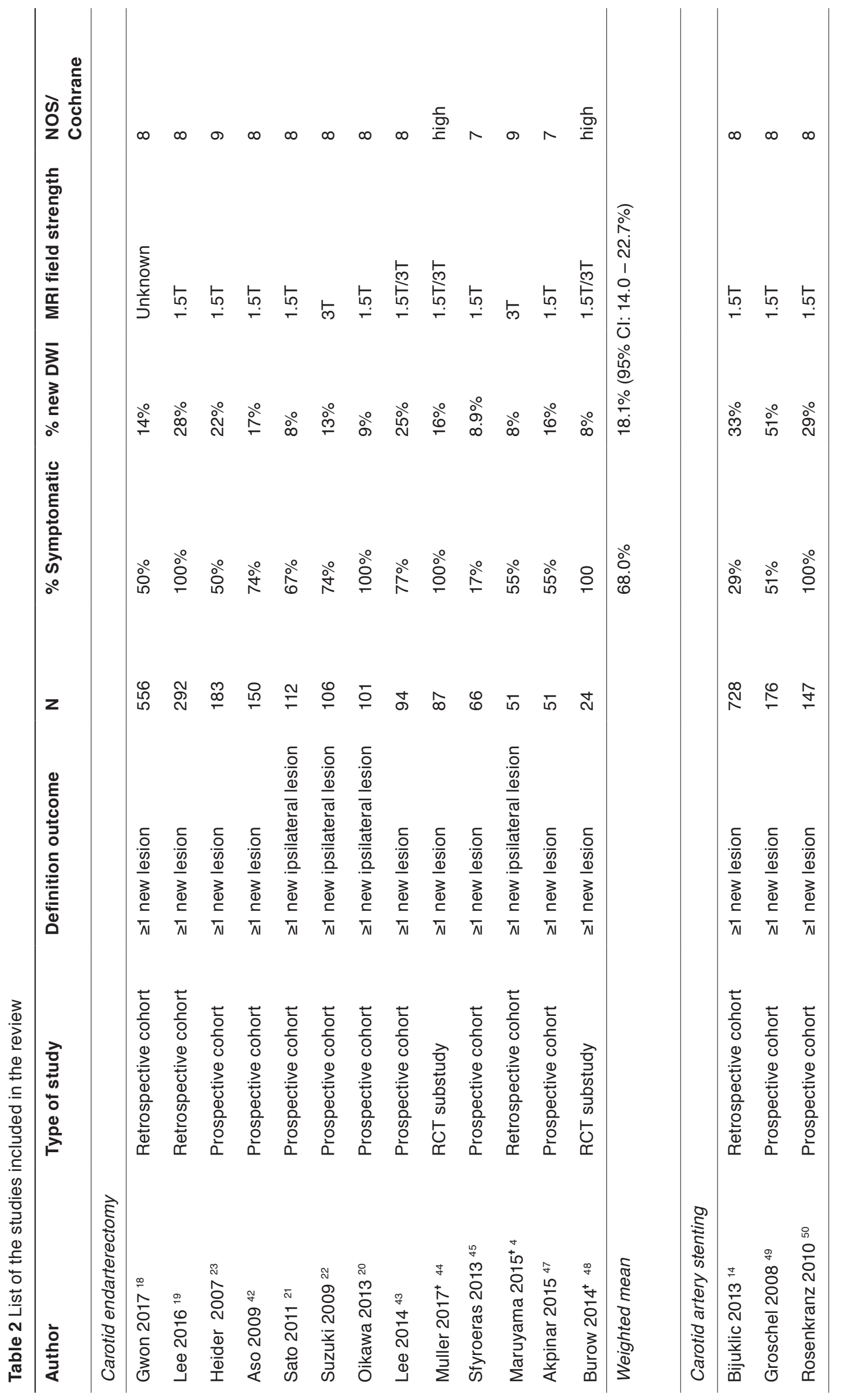


Part I Chapter 3

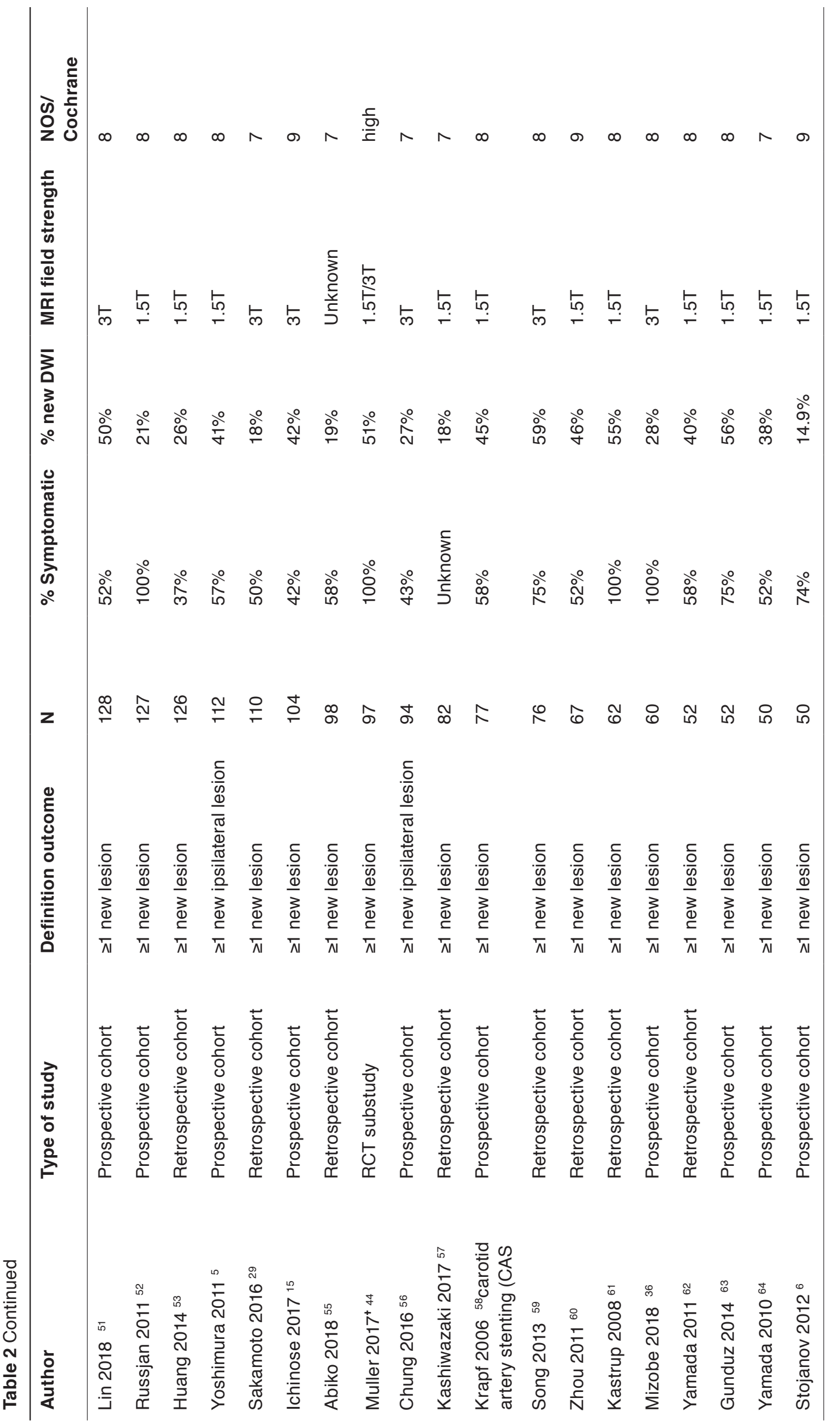




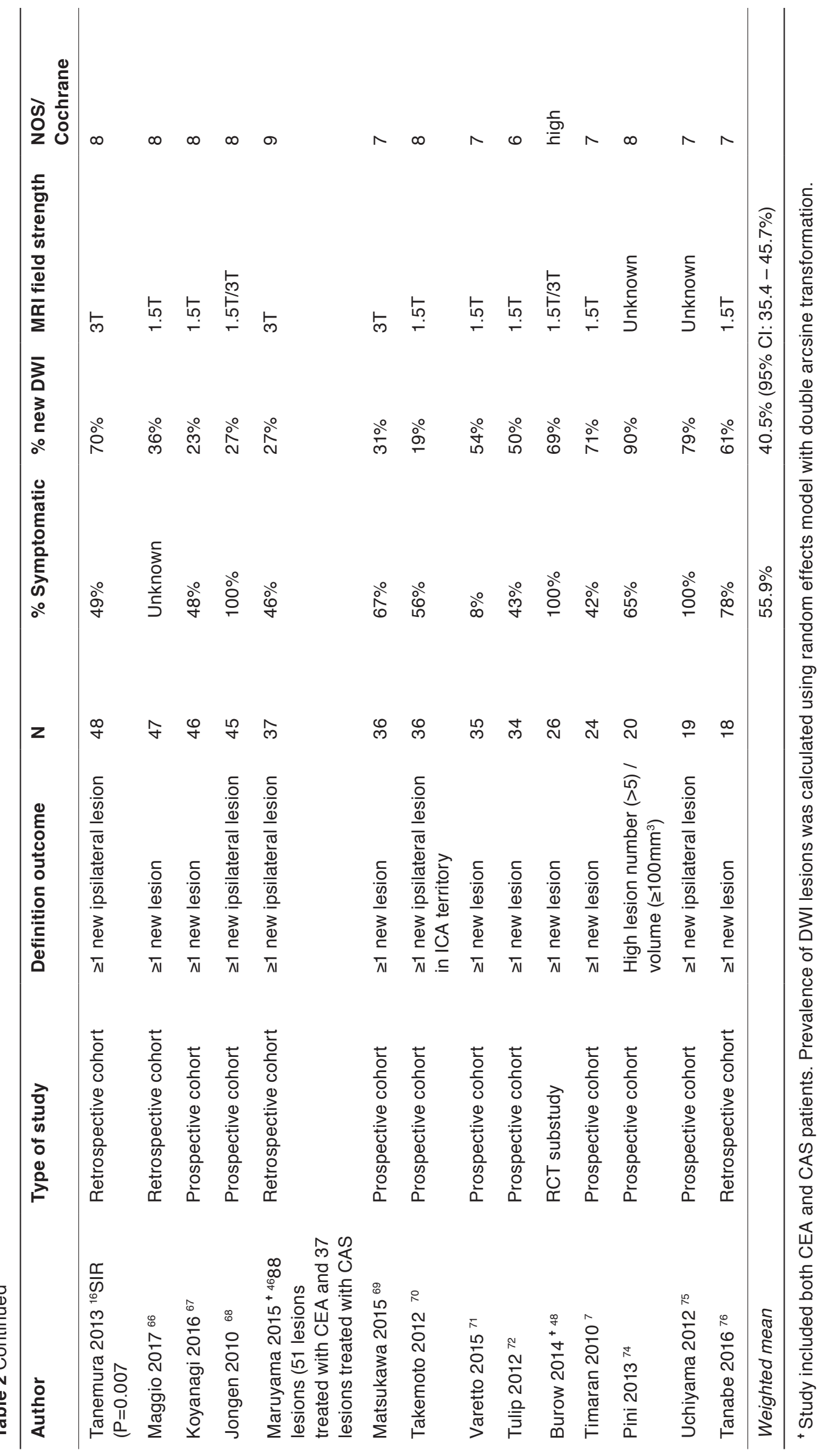




\section{CEA}

An overview of the reported predictive features is provided in table 3 , more detailed information on the individual study results can be found in the data supplement (III). None of the investigated features were negatively associated with new DWI lesions. All studies described either a positive association or found no association. To illustrate the relation between studies finding a positive association between the investigated parameter and presence of new DWI lesions and studies not finding a significant association, the results are plotted in figure 2a. Nine out of thirteen included articles reporting on CEA-patients investigated age, of which none reported a significantly higher chance of new DWI lesions with increasing age. None of the ten studies reporting on gender found a significant association (1696 patients) ${ }^{13}$. Symptomatic status (e.g. symptomatic vs asymptomatic) or recent stroke ( $<14$ days of intervention) was associated with an increased risk of new DWI lesions in only $2 / 9$ studies. However, these two studies represented a relatively large number of patients (848 out of 1661); see figure 2A. Cardiovascular risk factors and atherosclerotic burden (excluding ipsilateral carotid atherosclerosis) were never found to be significant predictors (respectively nine and six reporting articles). Eleven studies reported on various measures of plaque characteristics, two of them found a significant increased risk for development of new DWI lesions in case of high plaque vulnerability/high plaque burden (386 out of 1672 patients). All five articles investigating measures for cerebral haemodynamics, found an increased risk of new DWI lesions in case of preoperatively impaired cerebral haemodynamics (761 patients). Aortic arch and carotid anatomy were investigated in one study and was not associated with increased predisposition for new DWI lesions in CEA-patients. Three studies reported on markers of inflammation (hsCRP or CRP), of which 2 found to be independently associated with new DWI lesions (475 out of 526 patients). A marker of coagulation was once reported on (fibrinogen) and was found to be associated to an increased chance of new DWI lesions (183 patients). 


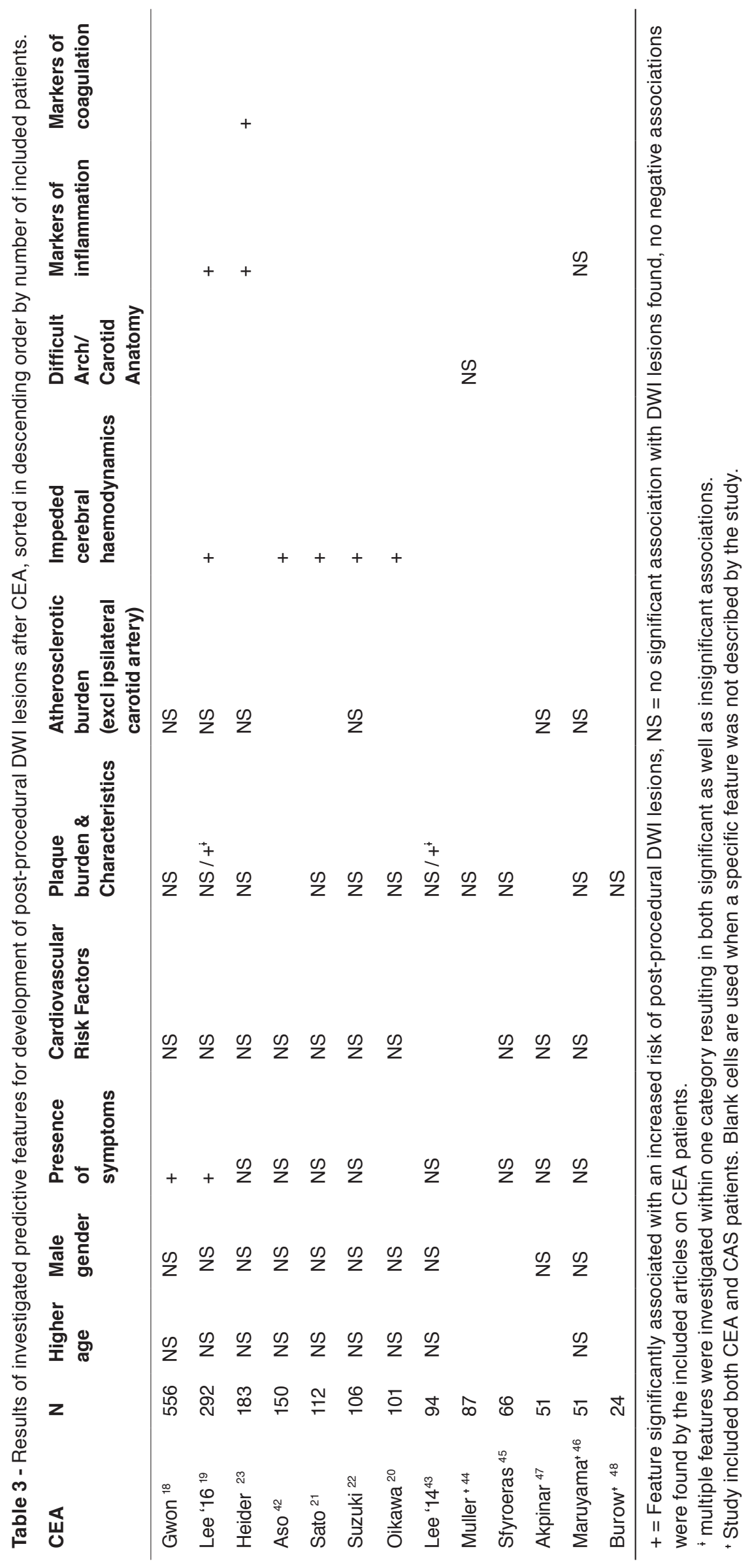




\section{CAS}

An overview of the reported predictive features and 36 articles reporting on CAS is given in table 4 and graphically represented in figure $2 \mathrm{~b}$. More detailed information can be found in the data supplement (III). Twenty-six studies (on 2849 patients) reported on age as a predictive feature and ten (1725 patients) described a significantly increased chance of new post-procedural DWI lesion in older patients. Of the twenty-two studies reporting on gender, only one study $(n=20)$ described a negative association between male gender and incidence of new lesions, others did not show a significant association. Symptomatic status was associated with an increased risk in four $(n=409)$ out of twenty-two studies investigating reporting on this topic $(n=2298)$.

Cardiovascular risk factors were reported on in twenty-four articles and was only associated with new DWI lesions in three studies (significant association with hypertension in one study, $n=728^{14}$ and significant association with LDL-cholesterol in two studies, $n=152^{15,16}$ ). Increased ipsilateral plaque burden or plaque vulnerability was often investigated $(n=3114)$ and frequently showed an increased chance of new DWI lesions $(n=2130)$. Within this category, signal intensity (ratio) and plaque volume/area was most often found to be predictive for new

DWI lesions. Features representative of atherosclerotic burden (other than ipsilateral carotid atherosclerosis) were investigated in twenty studies $(n=1467)$ and were found to be predictive for development of new lesion in three of them $(n=349)$. Impaired cerebral haemodynamics was found to be a risk factor for periprocedural DWI lesions in three $(n=143)$ out of five $(n=307)$ studies. Difficult carotid (large internal carotid artery angle) or arch-anatomy (aortic arch configuration type II or III) was associated with new lesions in $3(n=887)$ out of a total of five studies ( $n=957)$. Markers of inflammation and markers of coagulation were investigated less frequently and were found to have a significant association with presence of new DWI lesions in respectively $3 / 8$ and $1 / 2$ studies. 
Predictors of ischaemic brain lesions after carotid revascularization

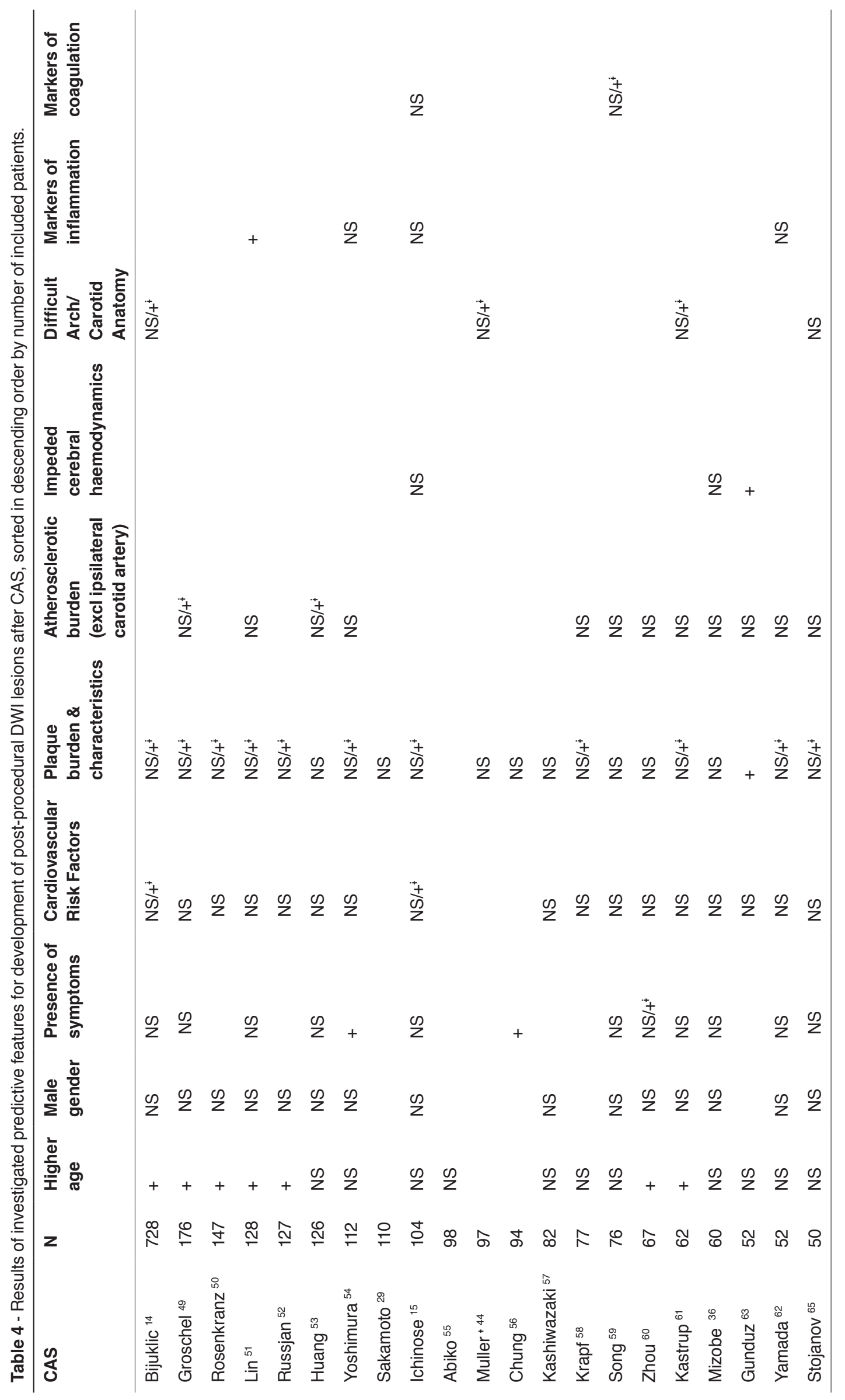


Part I Chapter 3

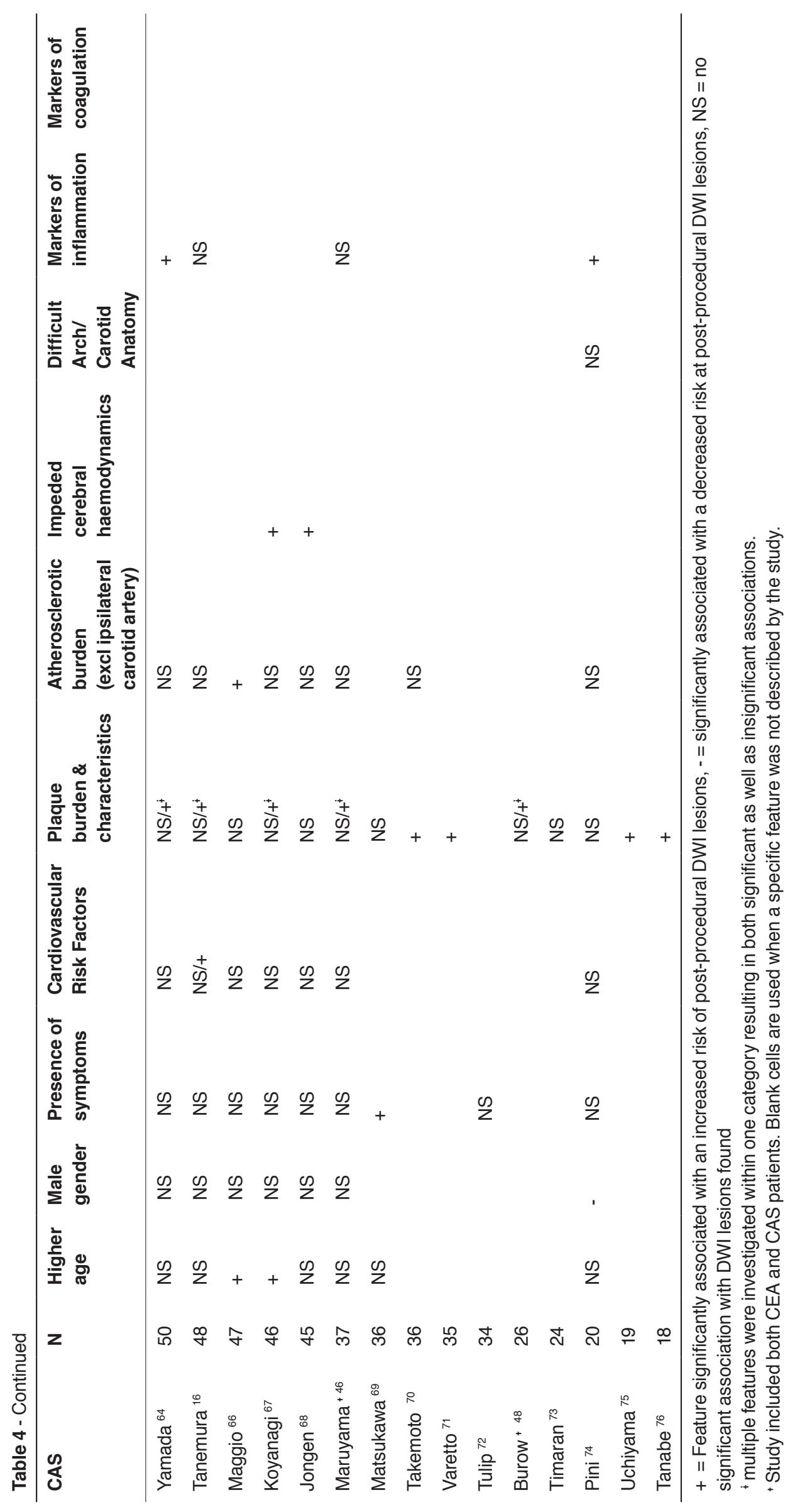




\section{Discussion}

In this review a systematic overview of the available evidence on predictive features for development of post-procedural DWI lesions after CEA (13 studies, 1873 patients) and CAS (36 studies, 3145 patients) was provided. For both CEA and CAS age, gender, cardiovascular risk factors, symptomatology, plaque burden/vulnerability, atherosclerotic burden, cerebrovascular haemodynamics, arch/carotid anatomy, inflammatory markers and markers of coagulation were investigated as potential predictive features. Factors associated with development of new DWI lesions were preprocedural symptoms, impeded cerebral haemodynamics and increased inflammatory markers in CEA-patients and age, plaque vulnerability and difficult vascular anatomy in CAS-patients.

Symptomatic patients are known to have a higher risk at post-procedural events compared to asymptomatic patients ${ }^{17}$. It is therefore not unexpected that a risk benefit for asymptomatic patients applies for development of DWI lesions as well ${ }^{18,19}$. CEA-patients with impeded preprocedural haemodynamics may be at risk during clamping of the carotid artery, since it may cause a critical reduction in cerebral perfusion. Only one of the included studies reporting on cerebral haemodynamics used routine intraluminal shunting ${ }^{19}$, others stated that no shunt was used ${ }^{20-22}$. It is unclear whether these studies used electrophysiological neuromonitoring to determine maintenance of sufficient cerebral perfusion after crossclamping of the carotid artery. Patients with an inadequate collateral blood-flow may be more at risk for ischaemic complications due to the impaired clearance of emboli theory. According to this concept, haemodynamic impairment may facilitate onset of ischaemia due to emboli generated from a proximal lesion ${ }^{8}$. Studies finding a positive association between increased inflammatory markers and new lesions used C-reactive protein (CRP) or highsensitive CRP as an inflammatory marker ${ }^{19,23}$. These studies advocate that inflammation may be related to presence of macrophages and T-lymphocytes in the plaque which is associated to plaque instability. This hypothesis is strengthened by studies finding differences in levels of inflammatory markers between symptomatic and asymptomatic patients ${ }^{24}$. Nevertheless, according to our results, investigated features of plaque instability in CEA-patients were often found not to be associated with new DWI lesions.

Higher age as a periprocedural risk factor in CAS patients corresponds to literature describing increased risk for stroke after CAS with age acting as an effect modifier for outcome ${ }^{25,26}$. Furthermore, biobank studies have revealed that increased age is related to increased plaque vulnerability ${ }^{27}$. This review showed that high plaque vulnerability was associated with new DWI lesions in CAS-patients. Vulnerable plaques, identified by presence of intraplaque haemorrhage, lipid-rich necrotic core and rupture of the fibrous cap are known to cause more ischaemic strokes or TIA in carotid stenosis patients ${ }^{28}$. They are also thought to be risk factors for embolic complications after CAS, by distal embolism into intracranial arteries as a result of debris migration during the procedure ${ }^{29}$. The same mechanism may apply for development of DWI lesions in CAS-patients. Increased tortuosity of the supra-aortic arteries has been associated with a higher risk of stroke/death within 30 days of $\mathrm{CAS}^{30}$, however randomized trial evidence comparing CEA and CAS in terms of clinical outcome is lacking. Complex 
anatomy of the supra-aortic arteries increases technical challenges during stenting procedure and repeated attempts to advance the catheter and guidewire may cause dislodgement of thrombotic material. An ICSS-substudy also included in this review demonstrated that patients with a complex configuration of the aortic arch and ICA tortuosity increase the risk of DWI lesions in CAS, but not CEA ${ }^{31}$.

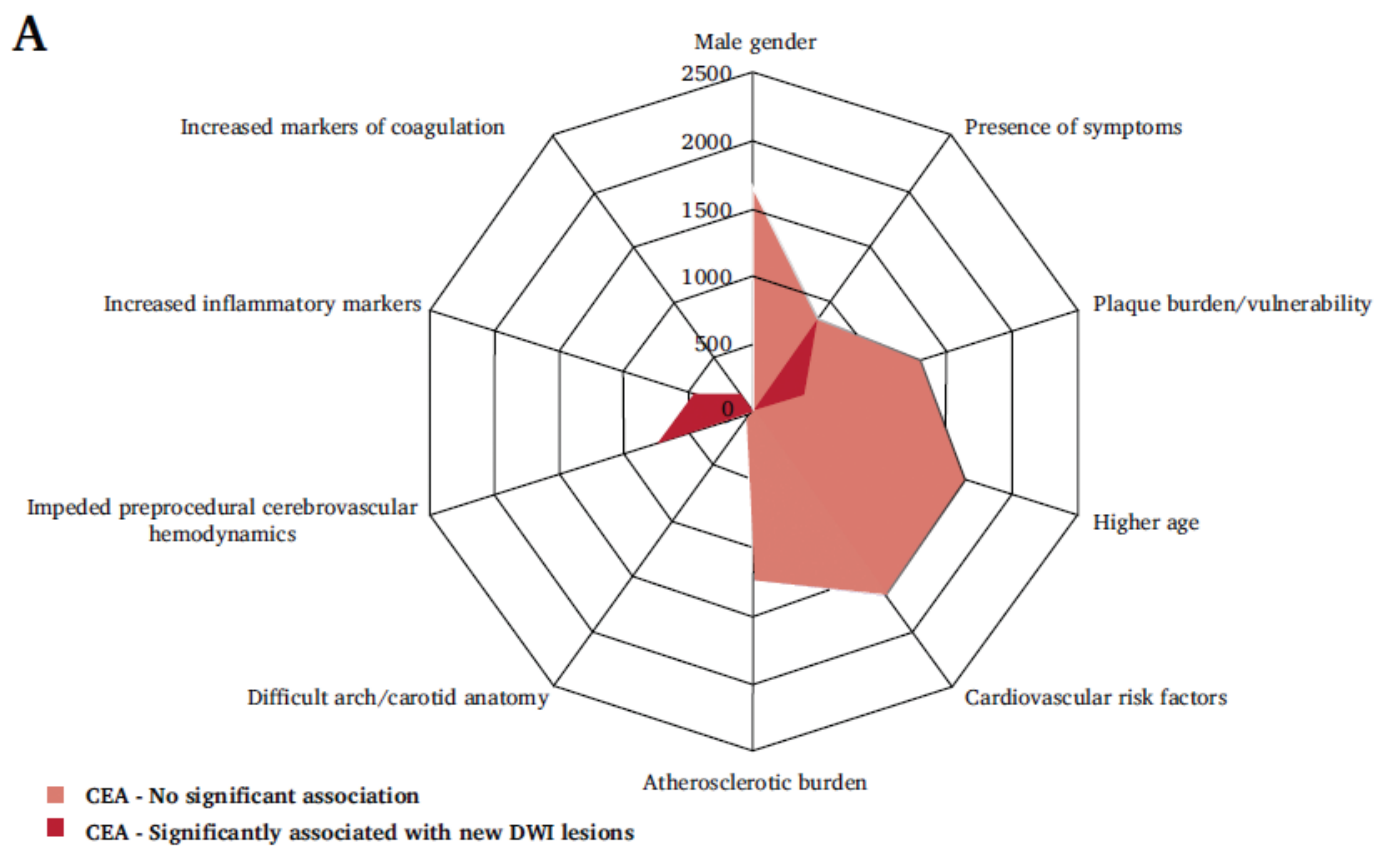

B

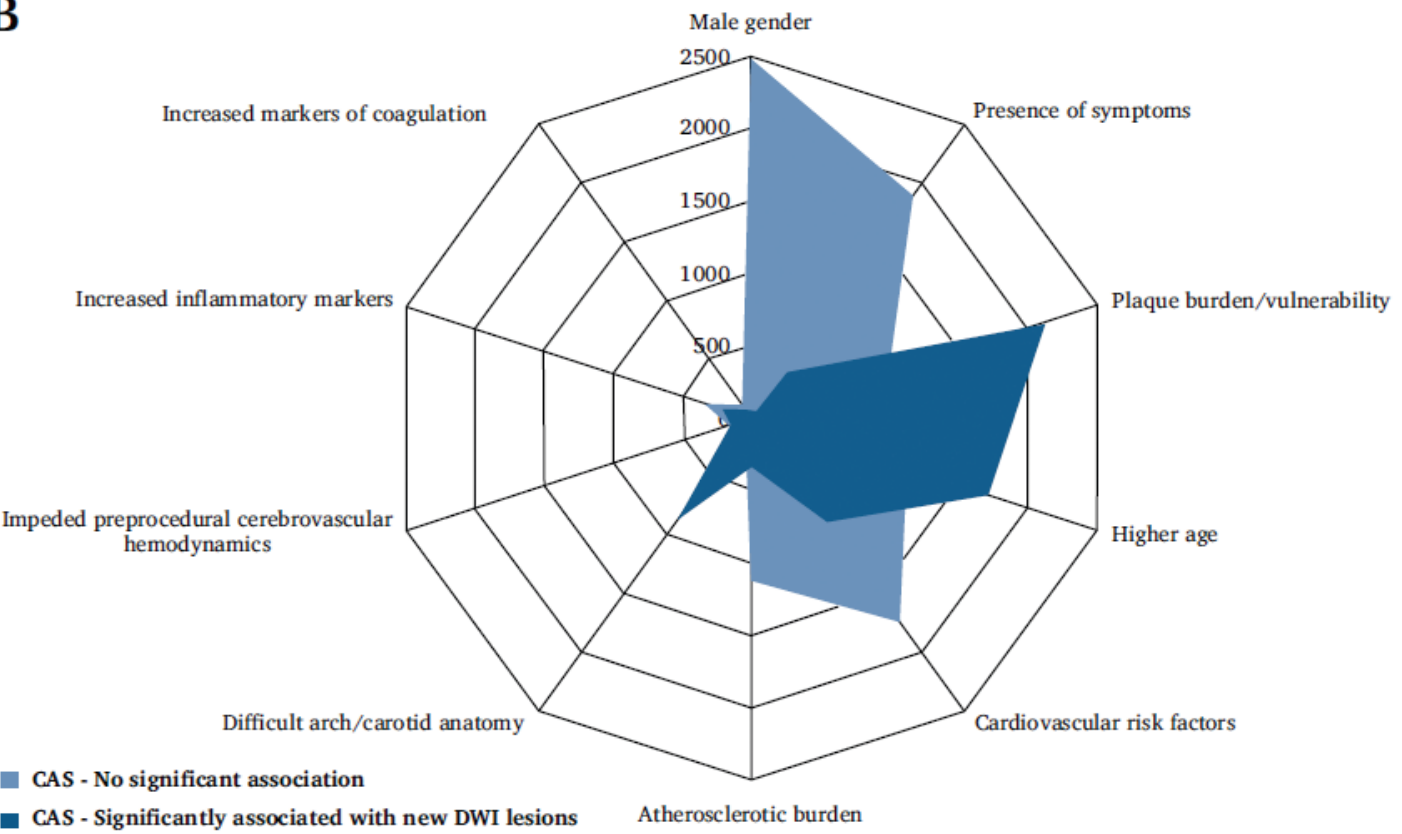

Figure 2 Radar graphs of the included prognostic features for CEA (figure A) and CAS (figure B). Studies that found a significant association (dark colored) were plotted against studies not finding a significant association (light colored). The y-axis represents the cumulative number of patients from the studies; each axis of the circular radar graph represents one prognostic category. 
The pooled prevalence of $18.1 \%$ new DWI lesions in CEA-patients as described in table 2 is equivalent to that described in two RCT's comparing CEA and CAS for prevalence of new DWI lesions (18\%-25\%) $)^{3,32}$. The pooled prevalence of $40.5 \%$ in CAS patients however, seems to be lower than reported in both trials (50 and $49 \%$ ). This may be partly explained by the fact that the study sample in one of these trials involved only symptomatic patients ${ }^{3}$ whereas we included studies on both symptomatic and asymptomatic patients. Another possible explanation for the differences in prevalence is the change over time in stent design and type of cerebral protection device as well as changes in antithrombotic and lipid-lowering therapy ${ }^{33-36}$. Moreover, patients with a deemed high-risk anatomy were more likely to be treated conservatively over the more recent years, resulting in a shift of patient selection. At last, in light of evidence suggesting that very urgent intervention may increase procedural risk due to presumed plaque vulnerability, procedural timing has been planned more carefully ${ }^{37,38}$. In previous studies the advantage of CEA over CAS with respect to prevention of new DWI lesions has already been demonstrated ${ }^{2,3}$. Although a meta-analysis of European RCT data showed that CAS was associated with significantly higher risk of procedural death/stroke risk after 30 days compared to CEA, follow-up results of these RCT's show that CAS appears to be as durable as CEA following the first 30 days ${ }^{39}$. Ongoing advances in stenting technology and safety may lead to a point where CAS is an equivalent treatment option compared to CEA in terms of major adverse events. The risk of perioperative DWI lesions has become increasingly important since these lesions are associated with a higher risk for recurrent cerebrovascular events ${ }^{4,5}$. As perioperative stroke risk has gradually decreased over the years, the necessity for a surrogate marker to assess possible effects of changes in treatment is growing. Subtle perioperative events such as micro-embolisms or minor decrease in cerebral perfusion may not be enough to cause stroke but can lead to silent ischaemic lesions. Decision making on type of intervention may be supported by patient profiling based on the investigated features. Secondly, as secondary stroke prevention has changed considerably (widespread use of statins and lower blood pressure targets), the discussion on invasive treatment versus BMT has re-emerged, especially in patients with a low risk of future cerebrovascular events. In light of this debate, the risk of development of periprocedural DWI lesions may contribute to an individualized risk-benefit assessment.

This review focused on pre-procedural risk assessment for development of DWI lesions. Naturally, treatment type and procedural conditions influence the likelihood of new DWI lesions greatly and we want to emphasize on the possible effect of choice of interventional techniques and medication on the risk of new DWI lesions. A recent review on cerebral protection, described several studies reporting a higher prevalence of DWI lesions in patients treated with cerebral protection compared to those treated without. Extensive comparison of type of protection strategy (proximal occlusion, distal occlusion and filter type) did not lead to any evincive evidence of a superiority of one of these protection devices in terms of DWI lesions ${ }^{34}$. A meta-analysis investigating stent-design found no differences in terms of major adverse events but found an increased risk for development of periprocedural DWI lesions in patients treated with open cell stenting ${ }^{40}$. Administration of additional preprocedural anticoagulants and additional lipid lowering therapy may be protective for development of new DWI lesions ${ }^{35,36,41}$. 
Another topic of interest is procedural timing in symptomatic patients ${ }^{37}$. As cerebrovascular events are more likely to occur in patients with instable plaque, one could rationalize that timing of the procedure in symptomatic patients may influence the chance of dislodgement of plaque debris and therefore new DWI lesions, in line with research suggesting increased stroke risk after very urgent $C E A^{38}$. Future research on timing of intervention should incorporate presence of DWI lesions as one of their secondary outcome measures.

\section{Limitations}

Most of the included studies were retrospective or prospective cohort studies in which the reason for selection for either one of the interventions (CEA or CAS) varied and may have been based on patient characteristics such as age and comorbidities or the physician's preference, introducing selection bias. To account for inherit differences we deliberately chose not to make a direct comparison between the groups. Future research should focus on identifying risk factors for DWI lesions after both CEA and CAS in patients with comparable characteristics. There's a wide heterogeneity in the investigated parameters, even within one type of predictive category. Few studies investigated the same parameter using consistent imaging modalities when evaluating for example plaque instability or cerebral haemodynamics. Although both of these investigated categories appear promising tools for identification of patients at risk for development of new DWI lesions, we were not able to select one specific instrument for risk assessment for this heterogeneous data collection and quantify the risk. Moreover, we were not able to analyse symptomatic and asymptomatic patients separately as these data were generally not provided by the included studies. As optimal treatment strategy for asymptomatic patients has yet to be determined, assessment of predictive parameters for DWI lesions in this specific category could be especially helpful. Future research should focus on development of a risk prediction-model to assist in decision making on type of revascularization.

Included studies used 1.5T, 3T or a mix of both MR field strengths. Previous studies have shown differences in detection rate of DWI lesions after CEA/CAS of the carotid artery between both field strengths ${ }^{3}$. As most studies used only one type of field strength, this is not likely to have caused a bias in the results of the individual studies. Reasonably, studies using 1.5T may have underreported silent ischaemic lesions, although pooling of studies based on MRI field strength did not result in a lower prevalence of DWI lesions in these studies compared to studies using $3 \mathrm{~T}$ MRI.

The majority of studies used presence of new DWI lesions as their primary endpoint, however some mentioned DWI only as a secondary outcome measure. These studies were likely to be underpowered to detect a statistically significant difference in DWI lesions for the investigated feature. Especially CEA-studies, in which the prevalence of DWI is lower may have suffered from this statistical problem. Pooling of data by performing an individual patient data metaanalysis may offer a solution to this problem, unfortunately this was not feasible due to the large heterogeneity of the investigated predictive features. 


\section{Conclusion}

Risk factors associated with the development of new DWI lesions after carotid stenting or endarterectomy are heterogeneous. For CEA-patients, assessment of symptom status, preprocedural cerebral haemodynamics and inflammatory parameters may help to identify those at risk for new DWI lesions. In CAS-patients, age and imaging of plaque characteristics and anatomy of supra-aortic arteries provides information on the susceptibility to new DWI lesions. These predictive features may assist in risk assessment to determine the indication for and optimal type of treatment being either medical intervention, carotid artery stenting or endarterectomy as revascularization strategy. 


\section{References}

1. Schnaudigel S, Gröschel K, Pilgram SM, Kastrup A. New brain lesions after carotid stenting versus carotid endarterectomy: a systematic review of the literature. Stroke. 2008;39(6):1911-9.

2. Gensicke H, Zumbrunn T, Jongen LM, Nederkoorn PJ, MacDonald S, Gaines PA, et al. Characteristics of ischemic brain lesions after stenting or endarterectomy for symptomatic carotid artery stenosis: Results from the international carotid stenting study-magnetic resonance imaging substudy. Stroke. 2013;44(1):80-6.

3. Bonati LH, Jongen LM, Haller S, Flach HZ, Dobson J, Nederkoorn PJ, et al. New ischaemic brain lesions on MRI after stenting or endarterectomy for symptomatic carotid stenosis: a substudy of the International Carotid Stenting Study (ICSS). Lancet Neurol. 2010;9(4):353-62.

4. Gensicke H, van der Worp HB, Nederkoorn PJ, Macdonald S, Gaines PA, Van Der Lugt A, et al. Ischemic brain lesions after carotid artery stenting increase future cerebrovascular risk. J Am Coll Cardiol. 2015 Feb;65(6):521-9.

5. Wolf O, Heider P, Heinz M, Poppert H, Schmidt-Thieme T, Sander D, et al. Frequency, clinical significance and course of cerebral ischemic events after carotid endarterectomy evaluated by serial diffusion weighted imaging. Eur J Vasc Endovasc Surg. 2004 Feb;27(2):167-71.

6. Pendlebury ST, Rothwell PM. Prevalence, incidence, and factors associated with pre-stroke and poststroke dementia: a systematic review and meta-analysis. Lancet Neurol. 2009;8(11):1006-18.

7. Gray WA. Flights from wonder: The search for meaning in diffusion-weighted brain lesions. J Am Coll Cardiol. 2015;65(6):530-2.

8. Caplan LR, Hennerici M. Impaired clearance of emboli (washout) is an important link between hypoperfusion, embolism, and ischemic stroke. Arch Neurol. 1998;55(11):1475-82.

9. Moher D, Liberati A, Tetzlaff J, Altman DG, Group TP. Preferred Reporting Items for Systematic Reviews and Meta-Analyses: The PRISMA Statement. PLoS Med. 2009;6(7):1-6.

10. Ottawa Hospital Research. The Newcastle-Ottawa Scale (NOS) for assessing the quality of nonrandomised studies in meta-analyses.

11. Higgins J, Altman D, Gotzsche P, Juni P, Moher D, Oxman a D. The Cochrane Collaboration's tool for assessing risk of bias in randomised trials. BMJ. 2011;343:5928-5928.

12. Barendregt JJ, Doi SA, Lee YY, Norman RE, Vos T. Meta-analysis of prevalence. J Epidemiol Community Health. 2013;67(11):974-8.

13. Lee C-Y, Kim H-W, Kim HR, Kim J-H, Seo J-H. Isolated central retinal artery occlusion (CRAO) without other thromboembolic event after carotid artery stenting (CAS). Interv Neuroradiol. 2016;22:24.

14. Bijuklic K, Wandler A, Varnakov Y, Tuebler T, Schofer J. Risk factors for cerebral embolization after carotid artery stenting with embolic protection: a diffusion-weighted magnetic resonance imaging study in 837 consecutive patients. Circ Cardiovasc Interv. 2013 Jun;6(3):311-6.

15. Ichinose N, Hama S, Tsuji T, Soh Z, Hayashi H, Kiura Y, et al. Predicting ischemic stroke after carotid artery stenting based on proximal calcification and the jellyfish sign. J Neurosurg. $2017 \mathrm{Jul} ; 1-9$.

16. Tanemura H, Maeda M, Ichikawa N, Miura Y, Umeda Y, Hatazaki S, et al. High-risk plaque for carotid artery stenting evaluated with 3-dimensional T1-weighted gradient echo sequence. Stroke. 2013 Jan;44(1):105-10.

17. Lokuge K, de Waard DD, Halliday A, Gray A, Bulbulia R, Mihaylova B. Meta-analysis of the procedural risks of carotid endarterectomy and carotid artery stenting over time. Br J Surg. 2018;105(1):26-36.

18. Gwon JG, Kwon TW, Cho YP, Kang DW, Han Y, Noh M. Analysis of Risk Factors for Cerebral Microinfarcts after Carotid Endarterectomy and the Relevance of Delayed Cerebral Infarction. J Clin Neurol. 2017 Jan;13(1):32-7.

19. Lee E-J, Cho Y-P, Lee S-H, Lee JS, Nam HJ, Kim BJ, et al. Hemodynamic tandem intracranial lesions on magnetic resonance angiography in patients undergoing carotid endarterectomy. J Am Heart Assoc. 2016 Oct;5(10).

20. Oikawa K, Ogasawara K, Saito H, Yoshida K, Saura H, Sato Y, et al. Combined measurement of cerebral and cerebellar blood flow on preoperative brain perfusion SPECT imaging predicts development of new cerebral ischemic events after endarterectomy for symptomatic unilateral cervical carotid stenosis. Clin Nucl Med. 2013 Dec;38(12):957-61.

21. Sato Y, Ogasawara K, Kuroda H, Suzuki T, Chida K, Fujiwara S, et al. Preoperative central benzodiazepine receptor binding potential and cerebral blood flow images on SPECT predict development of new cerebral ischemic events and cerebral hyperperfusion after carotid endarterectomy. J Nucl Med. 2011 Sep;52(9):1400-7.

22. Suzuki T, Ogasawara K, Hirooka R, Sasaki M, Kobayashi M, Ishigaki D, et al. Preoperative single-slab 3D time-of-flight magnetic resonance angiography predicts development of new cerebral ischemic events after carotid endarterectomy. Clinical article. J Neurosurg. 2009 Jul;111(1):141-6. 
23. Heider P, Poppert H, Wolf O, Liebig T, Pelisek J, Schuster T, et al. Fibrinogen and high-sensitive C-reactive protein as serologic predictors for perioperative cerebral microembolic lesions after carotid endarterectomy. J Vasc Surg. 2007 Sep;46(3):449-54.

24. Rerkasem K, Shearman CP, Williams JA, Morris GE, Phillips MJ, Calder PC, et al. C-reactive protein is elevated in symptomatic compared with asymptomatic patients with carotid artery disease. Eur $\mathrm{J}$ Vasc Endovasc Surg. 2002;23(6):505-9.

25. Voeks JH, Howard G, Roubin GS, Malas MB, Cohen DJ, Sternbergh WC, et al. Age and outcomes after carotid stenting and endarterectomy: The Carotid Revascularization Endarterectomy Versus Stenting Trial. Stroke. 2011;42(12):3484-90.

26. Howard G, Roubin GS, Jansen O, Hendrikse J, Halliday A, Fraedrich G, et al. Association between age and risk of stroke or death from carotid endarterectomy and carotid stenting: A meta-analysis of pooled patient data from four randomised trials. Lancet. 2016;387(10025):1305-11.

27. Van Lammeren GW, Reichmann BL, Moll FL, Bots ML, De Kleijn DPV, De Vries JPPM, et al. Atherosclerotic plaque vulnerability as an explanation for the increased risk of stroke in elderly undergoing carotid artery stenting. Stroke. 2011;42(9):2550-5.

28. Gupta A, Baradaran H, Schweitzer AD, Kamel H, Pandya A, Delgado D, et al. Carotid plaque MRI and stroke risk: A systematic review and meta-analysis. Stroke. 2013;44(11):3071-7.

29. Sakamoto S, Kiura Y, Okazaki T, Shinagawa K, Ishii D, Ichinose N, et al. Carotid artery stenting for vulnerable plaques on MR angiography and ultrasonography: utility of dual protection and blood aspiration method. J Neurointerv Surg. 2016 Oct;8(10):1011-5.

30. Naggara O, Touzé E, Beyssen B, Trinquart L, Chatellier G, Meder JF, et al. Anatomical and technical factors associated with stroke or death during carotid angioplasty and stenting: Results from the endarterectomy versus angioplasty in patients with symptomatic severe carotid stenosis (EVA-3S) trial and systematic review. Stroke. 2011;42(2):380-8.

31. Müller M, Von Hessling Van Der Lugt A, Doig D, Nederkoorn PJ, Macdonald S, Lyrer PA, et al. The risk of cerebral ischaemia in carotid artery stenting is associated with vascular anatomy - Results from the ICSS-MRI substudy. Cerebrovasc Dis. 2014;37:349.

32. Kuliha M, Roubec M, Procházka V, Jonszta T, Hrbáč T, Havelka J, et al. Randomized clinical trial comparing neurological outcomes after carotid endarterectomy or stenting. Br J Surg. 2015;102(3):194-201.

33. Yamada R, Guimaraes M, Adams J, Schonholz C. New technologies for CAS that might overcome the burden of microembolization. J Cardiovasc Surg (Torino). 2015 Dec;56(6):859-65.

34. Vos JA. Evidence overview: benefit of cerebral protection devices during carotid artery stenting. $J$ Cardiovasc Surg (Torino). 2017 Apr;58(2):170-7.

35. Patti G, Tomai F, Melfi R, Ricottini E, Macri M, Sedati P, et al. Strategies of clopidogrel load and atorvastatin reload to prevent ischemic cerebral events in patients undergoing protected carotid stenting. Results of the randomized ARMYDA-9 CAROTID (Clopidogrel and Atorvastatin Treatment During Carotid Artery Stenting) study. J Am Coll Cardiol. 2013 Apr;61(13):1379-87.

36. Mizobe T, Nakamura M, Motooka Y, Ashida N, Sugihara M. Impact of Additional Lipid-Lowering Therapy on New Ischemic Lesions of Diffusion-Weighted Imaging in Carotid Artery Stenting. J Stroke Cerebrovasc Dis. 2018;27(3):764-70.

37. Meershoek AJA, de Borst GJ. Timing of carotid intervention. Br J Surg. 2018;105(10):1231-3.

38. Strömberg S, Gelin J, Österberg T, Bergström GML, Karlström L, Österberg K. Very urgent carotid endarterectomy confers increased procedural risk. Stroke. 2012;43(5):1331-5.

39. De Borst GJ, Naylor AR. In the end, it all comes down to the beginning! Eur J Vasc Endovasc Surg. 2015;50(3):271-2.

40. de Vries EE, Meershoek AJA, Vonken EJ, Ruijter HM den, Berg JC van den, Borst GJ de, et al. Effect of stent design on clinical and radiological outcomes of carotid artery stenting: a meta-analysis. In-press. J Vasc Surg.

41. Nakagawa I, Park HS, Wada T, Yokoyama S, Yamada S, Motoyama Y, et al. Efficacy of cilostazol-based dual antiplatelet treatment in patients undergoing carotid artery stenting. Neurol Res. 2017 Aug;39(8):695701.

42. Aso K, Ogasawara K, Sasaki M, Kobayashi M, Suga Y, Chida K, et al. Preoperative cerebrovascular reactivity to acetazolamide measured by brain perfusion SPECT predicts development of cerebral ischemic lesions caused by microemboli during carotid endarterectomy. Eur J Nucl Med Mol Imaging. 2009 Feb;36(2):294-301.

43. Lee JH, Suh BY. Risk factor analysis of new brain lesions associated with carotid endarterectmy. Ann Surg Treat Res. 2014 Jan;86(1):39-44.

44. Muller MD, Ahlhelm FJ, von Hessling A, Doig D, Nederkoorn PJ, Macdonald S, et al. Vascular Anatomy Predicts the Risk of Cerebral Ischemia in Patients Randomized to Carotid Stenting Versus Endarterectomy. Stroke. 2017 May;48(5):1285-92. 
45. Sfyroeras GS, Bessias N, Moulakakis KG, Lyra S, Kotsikoris I, Andrikopoulos V, et al. New cerebral ischemic lesions after carotid endarterectomy. Ann Vasc Surg. 2013 Oct;27(7):883-7.

46. Maruyama D, Fukuda K, Kataoka H, Morita Y, Nishimura K, Kawamura Y, et al. Evaluation of carotid artery outward remodeling by $\mathrm{T} 1$-weighted magnetic resonance imaging in carotid endarterectomy and stenting. J Vasc Surg. 2015 Jun;61(6):1464-71.e1.

47. Akpinar MB, Sahin V, Sahin N, Abacilar AF, Kiris I, Uyar IS, et al. Previous chronic cerebral infarction is predictive for new cerebral ischemia after carotid endarterectomy. J Cardiothorac Surg. 2015 Nov;10:141.

48. Burow A, Lyrer PA, Nederkoorn PJ, Brown MM, Sztajzel R, Engelter ST, et al. Echographic risk index and cerebral ischemic brain lesions in patients randomized to stenting versus endarterectomy for symptomatic carotid artery stenosis. Ultraschall Med. 2014 Jun;35(3):267-72.

49. Groschel K, Ernemann U, Schnaudigel S, Wasser K, Nagele T, Kastrup A. A risk score to predict ischemic lesions after protected carotid artery stenting. J Neurol Sci. 2008 Oct;273(1-2):112-5.

50. Rosenkranz M, Thomalla G, Havemeister S, Wittkugel O, Cheng B, Krutzelmann A, et al. Older age and greater carotid intima-media thickness predict ischemic events associated with carotid-artery stenting. Cerebrovasc Dis. 2010;30(6):567-72.

51. Lin C, Tang X, Shi Z, Zhang L, Yan D, Niu C, et al. Serum tumor necrosis factor a levels are associated with new ischemic brain lesions after carotid artery stenting. J Vasc Surg. 2018;68(3):771-8.

52. Russjan A, Goebell E, Havemeister S, Thomalla G, Cheng B, Beck C, et al. Length of stenosis predicts treatment related ischemic lesions after carotid artery stenting. Cerebrovasc Dis. 2011;31:14.

53. Huang K-L, Chang Y-J, Chang C-H, Chang T-Y, Liu C-H, Hsieh I-C, et al. Impact of coexisting coronary artery disease on the occurrence of cerebral ischemic lesions after carotid stenting. PLoS One. 2014;9(4):e94280.

54. Yoshimura S, Yamada K, Kawasaki M, Asano T, Kanematsu M, Takamatsu M, et al. High-intensity signal on time-of-flight magnetic resonance angiography indicates carotid plaques at high risk for cerebral embolism during stenting. Stroke. 2011 Nov;42(11):3132-7.

55. Abiko M, Sakamoto S, Ochiai J, Yamada N, Kuroki K, Kurisu K. Applicability of Carotid Artery Stenting for Patients 80 Years or Older: A Single-Center Experience. World Neurosurg. 2018 Nov;119:e323-7.

56. Chung GH, Jeong JY, Kwak HS, Hwang SB. Associations between Cerebral Embolism and Carotid Intraplaque Hemorrhage during Protected Carotid Artery Stenting. AJNR Am J Neuroradiol. 2016 Apr;37(4):686-91.

57. Kashiwazaki D, Kuwayama N, Akioka N, Noguchi K, Kuroda S, D. K, et al. Carotid plaque with expansive arterial remodeling is a risk factor for ischemic complication following carotid artery stenting. Acta Neurochir (Wien). 2017 Jul;159(7):1299-304.

58. Krapf H, Nagele T, Kastrup A, Buhring U, Gronewaller E, Skalej M, et al. Risk factors for periprocedural complications in carotid artery stenting without filter protection: A serial diffusion-weighted MRI study. J Neurol. 2006 Mar;253(3):364-71.

59. Song TJ, Suh SH, Min PK, Kim DJ, Kim BM, Heo JH, et al. The influence of anti-platelet resistance on the development of cerebral ischemic lesion after carotid artery stenting. Yonsei Med J. 2013;54(2):288-94.

60. Zhou W, Zareie R, Tedesco M, Gholibeikian S, Lane B, Hernandez-Boussard T, et al. Risk factors predictive of carotid artery stenting associated subclinical microemboli. Int J Angiol. 2011;20(1):25-31.

61. Kastrup A, Groschel K, Schnaudigel S, Nagele T, Schmidt F, Ernemann U. Target lesion ulceration and arch calcification are associated with increased incidence of carotid stenting-associated ischemic lesions in octogenarians. J Vasc Surg. 2008 Jan;47(1):88-95.

62. Yamada K, Yoshimura S, Kawasaki M, Enomoto Y, Takano K, Asano T, et al. Prediction of silent ischemic lesions after carotid artery stenting using virtual histology intravascular ultrasound. Cerebrovasc Dis. 2011;32(2):106-13.

63. Gunduz Y, Akdemir R, Ayhan LT, Keser N. Can Doppler flow parameters of carotid stenosis predict the occurrence of new ischemic brain lesions detected by diffusion-weighted MR imaging after filter-protected internal carotid artery stenting? AJNR Am J Neuroradiol. 2014 Apr;35(4):760-5.

64. Yamada K, Kawasaki M, Yoshimura S, Enomoto Y, Asano T, Minatoguchi S, et al. Prediction of silent ischemic lesions after carotid artery stenting using integrated backscatter ultrasound and magnetic resonance imaging. Atherosclerosis. 2010 Jan;208(1):161-6.

65. Stojanov D, llic M, Bosnjakovic P, Zivkovic M, Jolic S, Vukasinovic N, et al. New ischemic brain lesions on diffusion-weighted MRI after carotid artery stenting with filter protection: frequency and relationship with plaque morphology. AJNR Am J Neuroradiol. 2012 Apr;33(4):708-14.

66. Maggio P, Altamura C, Lupoi D, Paolucci M, Altavilla R, Tibuzzi F, et al. The Role of White Matter Damage in the Risk of Periprocedural Diffusion-Weighted Lesions after Carotid Artery Stenting. Cerebrovasc Dis Extra. 2017 Jan;7(1):1-8.

67. Koyanagi M, Yoshida K, Kurosaki Y, Sadamasa N, Narumi O, Sato T, et al. Reduced cerebrovascular reserve is associated with an increased risk of postoperative ischemic lesions during carotid artery stenting. J Neurointerv Surg. 2016 Jun;8(6):576-80. 
68. Jongen LM, Hendrikse J, Moll FL, Mali WPTM, van der Worp HB. Cerebral perfusion affects the risk of ischemia during carotid artery stenting. Cerebrovasc Dis. 2010;29(6):538-45.

69. Matsukawa H, Fujii M, Uemura A, Suzuki K, Yamamoto D, Takahashi O, et al. Pathology of embolic debris in carotid artery stenting. Acta Neurol Scand. 2015 Apr;131(4):197-202.

70. Takemoto K, Ueba T, Takano K, Abe H, Hirata Y, Higashi T, et al. Quantitative evaluation using the plaque/ muscle ratio index panels predicts plaque type and risk of embolism in patients undergoing carotid artery stenting. Clin Neurol Neurosurg. 2013 Aug;115(8):1298-303.

71. Varetto G, Gibello L, Faletti R, Gattuso A, Garneri P, Castagno C, et al. Contrast-enhanced ultrasound to predict the risk of microembolization during carotid artery stenting. Radiol Med. 2015 Nov;120(11):1050-5.

72. Tulip HH, Rosero EB, Higuera AJ, Ilarraza A, Valentine RJ, Timaran CH. Cerebral embolization in asymptomatic versus symptomatic patients after carotid stenting. J Vasc Surg. 2012 Dec;56(6):1579-84; discussion 1584

73. Timaran $\mathrm{CH}$, Rosero EB, Martinez AE, Ilarraza A, Modrall JG, Clagett GP. Atherosclerotic plaque composition assessed by virtual histology intravascular ultrasound and cerebral embolization after carotid stenting. J Vasc Surg. 2010 Nov;52(5):1188-94.

74. Pini R, Faggioli G, Fittipaldi S, Pasquinelli G, Tonon C, Beltrandi E, et al. Inflammatory mediators and cerebral embolism in carotid stenting: new markers of risk. J Endovasc Ther. 2013 Oct;20(5):684-94.

75. Uchiyama N, Misaki K, Mohri M, Watanabe T, Hirota Y, Nakada M, et al. Association between carotid plaque composition assessed by multidetector computed tomography and cerebral embolism after carotid stenting. Neuroradiology. 2012 May;54(5):487-93.

76. Tanabe J, Tanaka M, Kadooka K, Hadeishi H. Efficacy of high-resolution cone-beam CT in the evaluation of carotid atheromatous plaque. J Neurointerv Surg. 2016 Mar;8(3):305-8. 


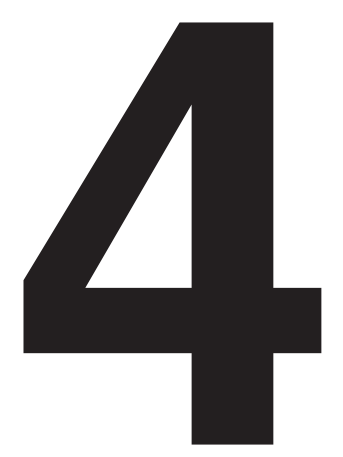

\section{Magnetic resonance imaging identified brain ischaemia in symptomatic patients undergoing carotid endarterectomy is related to histologically apparent intraplaque haemorrhage}

ML Rots, N Timmerman, DPV de Kleijn, G Pasterkamp, MM Brown, LH Bonati, GJ de Borst Eur J Vasc Endovasc Surg 2020;58(6):796-804 


\section{Abstract}

OBJECTIVES Intraplaque haemorrhage (IPH) has been independently associated with a higher risk of future ipsilateral stroke in patients with carotid artery stenosis. Evaluation of plaque characteristics may contribute to risk-assessment of recurrent (silent) cerebrovascular events in order to prioritize patients for timing of treatment. It is unknown if patients showing histologically apparent IPH also have increased risk of silent ischaemic brain lesions in the waiting period between index event and revascularization.

METHODS A retrospective analysis was performed based on prospectively collected data of patients included simultaneously in the MRI-substudy of the International Carotid Stenting Study and Athero-Express biobank. Patients randomized for carotid endarterectomy (CEA) were operated between 2003-2008. Brain MRI was performed 1-7 days prior to CEA. Plaques were histologically examined for presence of IPH. Primary outcome parameter was presence of silent ipsilateral brain-ischaemia on MR-DWI appearing hypo-/isointense on apparent diffusion coefficient.

RESULTS 53 patients with symptomatic carotid stenosis meeting study criteria were identified of which 13 showed $\geq 1$ recent ipsilateral DWI lesion on pre-operative scan. Time between latest ipsilateral neurological event and revascularization was 45 days (range 6-200) in DWInegative patients versus 34 days (range 6-74, $p=16$ ) in DWI-positive patients. IPH was present in 24/40(60.0\%) DWI-negative patients versus 12/13(92.3\%) DWI-positive patients (OR: 8.00; $95 \% \mathrm{Cl}: 0.95-67.7, p=0.056)$. Multivariate logistic regression analysis correcting for age and type of index-event revealed that IPH was independently associated with DWI lesions in the waiting period till surgery (OR:10.8; 95\% Cl:1.17-99.9, $p=.036$ ).

CONCLUSION Symptomatic patients with ipsilateral carotid stenosis and silent brain ischaemia on preoperative MR-DWI, more often showed pathological evidence of IPH compared to those without ischaemic lesions. This identifies carotid IPH as a marker for patients at risk for silent brain ischaemia and possibly for future stroke and other arterial disease complications. Such patients may be more likely to benefit from CEA than those without evidence of ipsilateral carotid IPH. 


\section{Introduction}

The presence of carotid artery intraplaque haemorrhage (IPH) is considered an important maker of plaque instability and associated with a high risk for clinically relevant events such as transient ischaemic attack (TIA) or stroke due to the tendency to rupture'. Signs of presence of carotid artery IPH on magnetic resonance imaging (MRI) or duplex have been associated with an increased risk of future cerebrovascular events and IPH is more common in symptomatic patients compared to asymptomatic patients ${ }^{2,3}$. Moreover, IPH is associated with increased risk of any type of secondary cardiovascular event in male patients such as (fatal) myocardial infarction (MI), (fatal) stroke, coronary and peripheral interventions, and cardiovascular death4 Risk assessment of recurrent cerebrovascular events based on plaque characteristics may be helpful for prioritizing patients for the timing of carotid revascularization. Current guidelines recommend that symptomatic patients with carotid stenosis should be considered (in case of $50-69 \%$ stenosis) or recommended (in case of $70-99 \%$ stenosis) for treatment within 14 days of the index event ${ }^{5}$. Nonetheless, these recommendations have been based on post hoc analyses of outdated RCT's and additionally may not always be feasible due to pre-hospital or in-hospital delay ${ }^{6}$. Identification of IPH may help to select patients that are most at risk for recurrent events and may help to select those in which urgent revascularization may be appropriate.

Ischaemic brain lesions on magnetic resonance diffusion weighted imaging (MR-DWI) are increasingly being used as a surrogate marker of ischaemic events for postoperative outcome after revascularization as they are associated with an increased risk of future cerebrovascular events ${ }^{7}$. To date, no studies have been performed that investigated the role of DWI lesions in the preoperative period specifically. One of the major advantages of assessing presence of new DWI lesions is that they may appear within few hours after a thrombo-embolic event and $\mathrm{DWI}$ is therefore sensitive to recent changes. By assessing diffusion restricted brain areas with MR-DWI in combination with apparent diffusion coefficient (ADC), recent infarction can be identified. ADC-maps may depict darkening within minutes of stroke onset and distinguishes stroke from "T2 shine through", which can be seen later after infarction and appears bright on DWI. Low signal intensity on ADC persists for about 7-10 days ${ }^{8}$. Until now, it is unclear if carotid plaque characteristics are associated with these silent brain lesions, identifying them as their potential source. For this, we investigated whether patients with characteristics of plaque instability have an increased risk of silent preprocedural ischaemic brain lesions during their waiting period for carotid intervention.

Since thrombo-embolic events are the most common underlying cause of ischaemic brain lesions in carotid patients, we hypothesize that patients showing histologically apparent signs of IPH in the atherosclerotic plaque (excised during carotid endarterectomy) are more likely to show (recurrent) silent ischaemic brain lesions in the waiting period between index event and revascularization. 


\section{Methods}

A retrospective analysis was performed based on prospectively collected data of patients included simultaneously in the MRI-substudy of the International carotid stenting study (ICSS) ${ }^{9}$ and the Athero-Express (AE) biobank study of the University Medical Center (UMC) Utrecht ${ }^{10}$. Patients that underwent carotid endarterectomy (CEA) between October 2003 and October 2008 were included with a median waiting time (defined as the number of days between the most recent event and surgery) of 44 days. Patients included in the ICSS were symptomatic (symptoms attributable to the randomized artery within twelve months before randomization) carotid stenosis patients with stenosis $>50 \%$ deemed to require treatment. Symptomatic patients included those with previous stroke (acute disturbance of focal neurological function attributed to vascular disease and of ischaemic origin, lasting more than 24h), TIA (acute disturbance of focal neurological function attributed to vascular disease with recovery within 24h) or amaurosis fugax (transient monocular blindness attributed to vascular disease with recovery within 24h). the following exclusion criteria were asserted: previous revascularization in the randomized artery, contraindications for either treatment and planned major surgery ${ }^{11}$. In course of the study protocol of the ICSS MRI-substudy, all patients underwent an additional MRI-scan 1-7 days before surgery for assessment of silent brain ischaemia. Carotid plaques were collected within the AE-biobank. No additional exclusion criteria were asserted for the AE-biobank study other than that the patient had to be randomized for CEA.

\section{MRI}

Primary outcome parameters include ipsilateral ischaemic lesions on MR-DWI appearing hypo-/isointense on apparent diffusion coefficient (ADC), and its correlation to intraplaque haemorrhage (see figure 1 for an illustrative timeline). Secondary outcome parameters included presence of white matter lesions (WML) semi-quantitatively assessed on FLAIR sequences by use of the age-related-white matter changes (ARWMC) score. White matter lesions and basal ganglia lesions were both assessed on a 0-3 point scale for the ipsilateral hemisphere and a sum-score was used for further data analyses(see supplemental table SI for exact definitions) ${ }^{12}$.

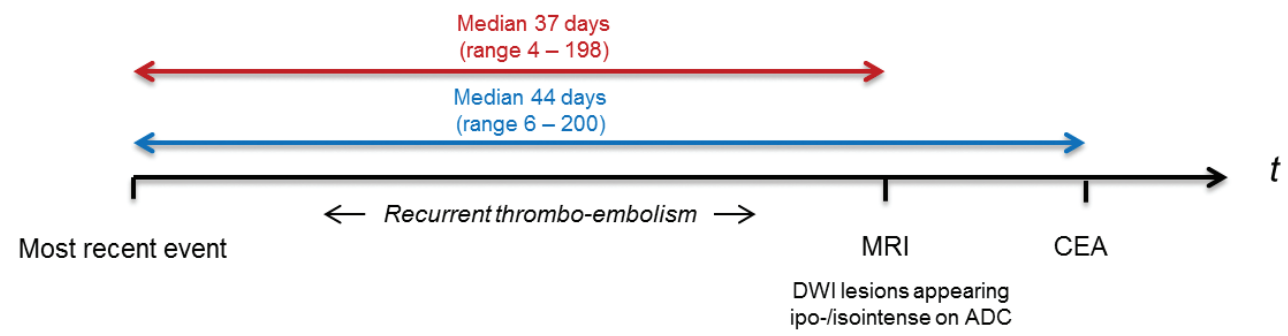

Figure 1 Timeline illustrating waiting time until revascularization. DWI, diffusion weighted imaging; ADC, apparent diffusion coefficient. Waiting time (indicated by blue arrow) was defined as the number of days between the most recent event (hemispheric/retinal stroke, TIA or amaurosis fugax) and CEA. The red arrow represents the time between latest symptom and preoperative MRI. 


\section{Atherosclerotic plaque assessment}

After CEA, all atherosclerotic plaques were immediately processed. The carotid plaque was cut into segments of 5-mm thickness along the longitudinal axis. The segment with the largest plaque burden was chosen as a culprit lesion and subjected to histological examination. $\mathrm{A}$ more detailed description can be found in the supplemental methods. The investigated plaque characteristics resulting from immunochemical staining were: presence of $\mathrm{PH}$, presence of lipid core $(\geq 40 \%)$, moderate/heavy calcifications, moderate/heavy collagen, mean number of microvessels per hotspot, percentage of positive macrophage staining per plaque and percentage of positive smooth muscle cell $(S M C)$ staining per plaque ${ }^{13}$. IPH was defined as the composite of plaque bleeding at the luminal side of the plaque as a result of plaque disruption and haemorrhage within the tissue of the plaque ${ }^{14}$.

\section{Clinical outcome}

Major cardiovascular events during 3-years of follow-up were reported, consisting of nonfatal MI, nonfatal stroke and cardiovascular death. MI was reported when at least two of the following criteria were present: (1)Chest pain for $\geq 20$ minutes, not disappearing after administration of nitrates; (2)ST-elevation $>1 \mathrm{~mm}$ in two following leads or a left bindle-branchblock on the electrocardiogram; (3)CK elevation of at least two times the normal value of CK and an $\mathrm{MB}$ fraction $>5 \%$ of the total $\mathrm{CK}$.

\section{Statistics}

A sample size of at least of 48 patients was required on the basis of detecting a difference in proportion of patients with IPH in the DWI-positive group of two times compared to the DWInegative group at a significance level of $.05,80 \%$ power, assuming $25 \%$ of patients having preoperative DWI lesions and $80 \%$ of DWI-positive patients having IPH. Data were inspected for patterns of missing values. The proportion of randomly missing values for baseline characteristics did not exceed $2 \%$. Differences in binary characteristics were analysed with Pearson's Chi square. Differences in continuous parameters were calculated with a student's $\mathrm{t}$-test when data were normally distributed and otherwise using a Mann-Whitney $U$ test. For the ARWMC score a binary outcome parameter was used based on median ipsilateral ARWMC score (sum score $\leq 2$ compared to sum score $>2$ ). To investigate independent associations between histological plaque characteristics and presence of fresh DWI lesions as well as ARWMC score, we conducted a multivariable logistics regression analysis correcting for any baseline characteristics with $p<0.1$ in univariate analysis. Age and type of qualifying event were also added to the multivariable models since these are considered as potential confounders based on earlier described associations with IPH ${ }^{3,15}$. In case of ARWMC score, estimated-packyears was identified as an additional potential confounder from univariate analysis and therefore included in multivariate analysis. Non-normally distributed quantitative histological parameters including number of microvessels and percentages of macrophage and SMC staining required logarithmic transformation before entering into regression models. SPSS 25.0 (SPSS Inc, Chicago, Illinois, USA) was used for all statistical analysis. 


\section{Results}

\section{Patient characteristics}

53 patients met the inclusion criteria of the ICSS-MRI substudy and were simultaneously included in the AE biobank study. Qualifying events were major stroke $(n=7)$, minor stroke $(n=11)$, cerebral TIA $(n=28)$ and amaurosis fugax $(n=7)$. Mean waiting time between the latest symptom (stroke/TIA) and CEA was 51 days (median 44, range 6-200 days)

\section{DWI/ADC lesions}

Thirteen (25\%) of 53 included patients had preoperative ipsilateral DWI lesions that appeared hypo- or isointense on ADC. Baseline characteristics of patients with and without recent DWI lesions are presented in table 1. No statistical differences in baseline features were found between the two groups. In patients with preoperative ipsilateral DWI lesions, mean number of DWI lesions was 2 (median 1, range 1-6). The mean time between the latest symptom and revascularization was 56 days (median 45, range 6-200 days) in the DWI-negative group and 36 days (median 34 , range 6-74 days, $p=0.16$ ) in the DWI-positive group. Mean time between latest symptom and MRI was 52 days (median 40, range 5-198) in DWI-negative patients versus 34 (median 33 , range $4-73, p=0.218$ ) in DWI-negative patients.

The investigated histological plaque characteristics are shown in table 2 for DWI-negative and DWI-positive patients. 24 out of $40(60.0 \%)$ DWI-negative patients showed IPH on histological assessment, versus 12 out of 13 (92.3\%) in DWI-positive patients (see example in figure 2). A lipid-core of $\geq 40 \%$ of the total plaque area was present more often in the DWI-negative group (23/40; $57.7 \%)$ compared to the DWI-positive group (3/13; 23.1\%). For other plaque characteristics results were similar between DWI-positive and DWI-negative patients. Of the total of 36 patients with IPH $12(33.3 \%)$ had preoperative DWI lesions. Of 17 patients without IPH $1(6 \%)$ showed DWI lesions. Univariate logistic regression showed an odds ratio (OR) of 8.0 (95\% Cl: $0.95-67.7, p=.056)$ for presence of DWI lesions in IPH-positive patients compared to IPH-negative patients. Multivariate logistic regression analysis with correction for age and type of index event revealed that IPH was independently associated with presence of DWI lesions in the waiting period till surgery (OR: 10.8; 95\% Cl:1.17-99.9, $p=.036$ ). Univariate analysis showed decreased odds for presence of DWI lesions in patients with a large (>40\%) lipid core (OR: $0.22 ; 95 \% \mathrm{Cl}: 0.05-0.93, p=.040$ ) which remained significant after correction for age and type of index event (OR:0.184; 95\%-Cl: 0.041-0.836, $p=.028)$. Other plaque characteristics did not show any significant association with development of DWI lesions in uni- or multivariate analysis.

In 53 patients a total of $28 \mathrm{DWI}$ lesions were detected of which 26 were ipsilateral and 2 were contralateral. Of the total cohort the mean number of DWI lesions was 0.5 (median 0 , range 0-6 lesions). Mean number of ipsilateral DWI lesions in IPH positive patients was 0.7 (median 0 , range $0-6$ ) versus 0.1 (median 0 , range $0-1)$ DWI lesions in IPH negative patients $(p=0.028$ ). Of the two patients that had a (single) contralateral DWI lesion, one was IPH positive and one was IPH negative. 
Table 1 Baseline characteristics of ipsilateral DWI-negative versus DWI-positive patients

\begin{tabular}{|c|c|c|c|}
\hline Patient characteristic & $\begin{array}{l}\text { DWI negative } \\
N=40\end{array}$ & $\begin{array}{l}\text { DWI positive } \\
N=13\end{array}$ & $p$ value \\
\hline Age, mean (std) & $68.2(8.6)$ & $69.3(8.4)$ & .673 \\
\hline Male gender, n (\%) & $28(70.0)$ & $11(84.6)$ & .299 \\
\hline Hypertension, n (\%) & $26(65.0)$ & $11(84.6)$ & .181 \\
\hline Systolic blood pressure, mean (std) & $163(29)$ & $158(21)$ & .716 \\
\hline Diastolic blood pressure, mean (std) & $85(15)$ & $80(13)$ & .260 \\
\hline Blood pressure medication use, $\mathrm{n}(\%)$ & $29(72.5)$ & $12(92.3)$ & .138 \\
\hline Diabetes Mellitus, n (\%) & $9(22.5)$ & $3(23.1)$ & .966 \\
\hline Hypercholesterolemia, n (\%) & $26(65.0)$ & $8(61.5)$ & .821 \\
\hline LDL, median (range) & $\begin{array}{l}2.50(0.91- \\
5.80)\end{array}$ & $\begin{array}{l}1.84(0.57- \\
4.50)\end{array}$ & .188 \\
\hline Statin use, n (\%) & $35(87.5)$ & $13(100)$ & .180 \\
\hline Antiplatelet use, n (\%) & $36(90)$ & $13(100)$ & .236 \\
\hline Oral anticoagulants, n (\%) & $6(15)$ & $1(7.7)$ & .499 \\
\hline Currently smoking, n (\%) & $15(37.5)$ & $3(23.1)$ & .340 \\
\hline Estimated pack-years, median (range) & $15.0(0-86)$ & $12.5(0-65)$ & .461 \\
\hline BMI, mean (std) & $25.8(3.7)$ & $25.0(2.9)$ & .520 \\
\hline History of PAOD, n (\%) & $8(20.0)$ & $2(15.4)$ & .712 \\
\hline History of CAD, n (\%) & $12(30.0)$ & $5(38.5)$ & .570 \\
\hline $\begin{array}{l}\text { Qualifying symptom = hemispheric stroke, } \mathrm{n}(\%) \\
\text { Major stroke, } \mathrm{n}(\%) \\
\text { Minor stroke, } \mathrm{n}(\%) \\
\text { Cerebral TIA, } \mathrm{n}(\%) \\
\text { Amaurosis fugax, } \mathrm{n}(\%)\end{array}$ & $\begin{array}{l}13(32.5) \\
3(7.5) \\
10(25.0) \\
21(52.5) \\
6(15.0)\end{array}$ & $\begin{array}{l}5(38.5) \\
4(30.8) \\
1(7.7) \\
7(53.8) \\
1(7.7)\end{array}$ & .693 \\
\hline Stenosis grade $\geq 70 \%, \mathrm{n}(\%)$ & $38(95.0)$ & $11(84.6)$ & .218 \\
\hline Waiting time (days), median (range) & $45(6-200)$ & $34(6-74)$ & .160 \\
\hline Time between event and MRI, median (range) & $40(5-198)$ & $33(4-73)$ & .218 \\
\hline
\end{tabular}

Data are given as proportion of the group (\%), as mean with standard deviation in case of normally distributed data, or as median with range in case of not normally distributed data. Hypertension; previously diagnosed by an MD or use of antihypertensive drugs. Systolic and diastolic blood pressure were measured at intake/ hospital admission. Blood pressure medication use; use of any or more antihypertensive drugs. Diabetes mellitus; previously diagnosed by an MD or use of antidiabetic medication. LDL; mmol/L measured within 1 month prior to surgery. Antiplatelet use; dipyridamole, acetylsalicylic, carbasalate calcium or clopidogrel, anticoagulation; coumarone or direct acting oral anticoagulant, PAOD; peripheral artery disease was defined as a history of peripheral interventions or intermittent claudication or ankle-brachial index<0.7. History of CAD; carotid artery disease was defined as a composite of angina pectoris, myocardial infarction, percutaneous coronary interventions or coronary bypass surgery. Stenosis grade $\geq 70 \%$; ipsilateral stenosis grade $\geq 70 \%$ as measured by NASCET criteria. Waiting time; number of days between the most recent event and CEA. Time between most recent event and MRI; number of days between the most recent preoperative cerebrovascular event and preoperative MRI. 
Part I Chapter 4

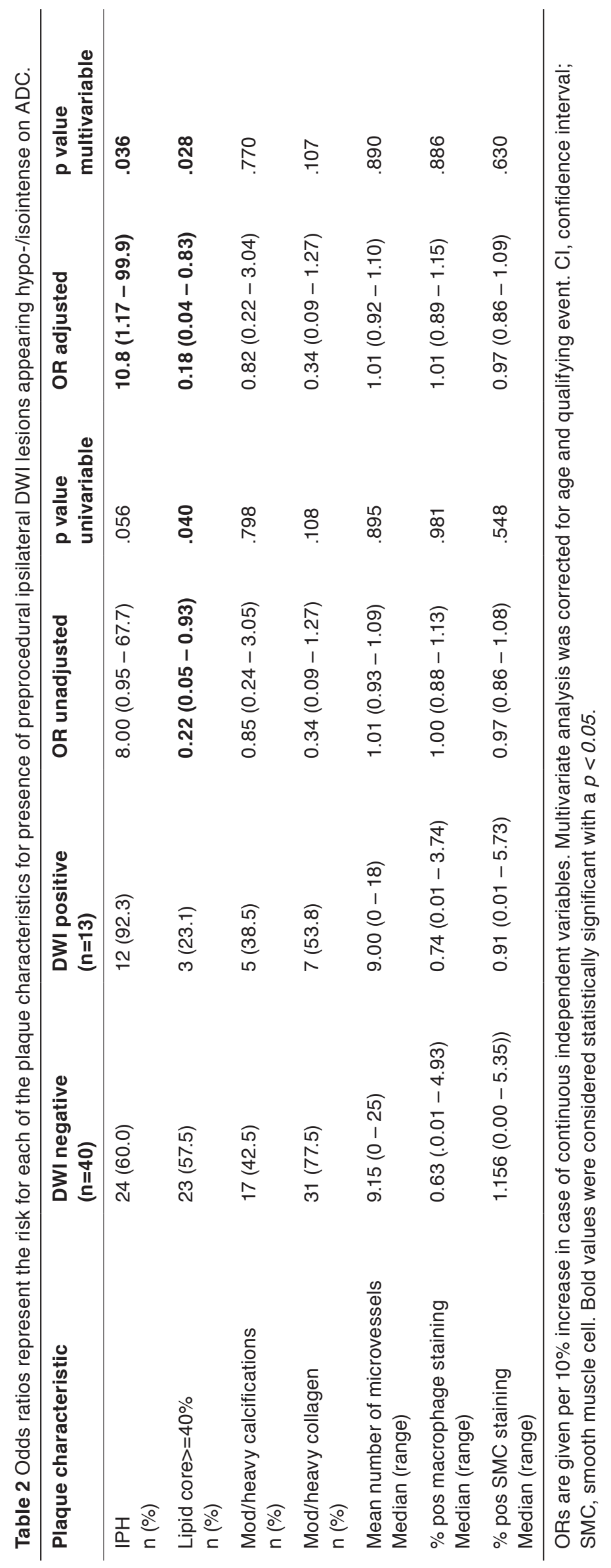



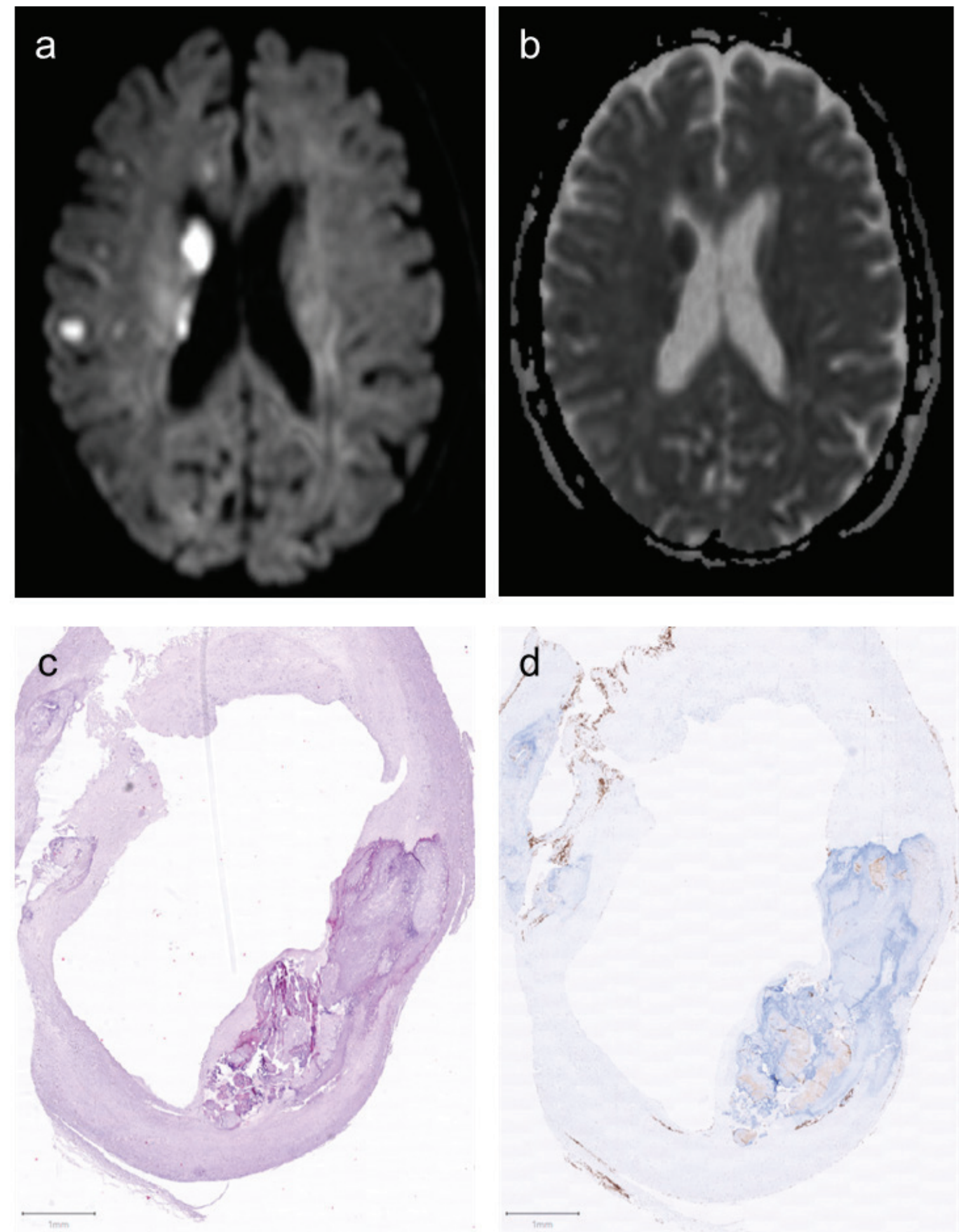

Figure 2 Typical example of patient presenting with minor stroke in right hemisphere and right-sited carotid stenosis. MRI just prior to CEA showed ipsilateral DWI lesions (a) appearing hypo-intense on ADC (b). Upon histological examination the culprit lesion showed intraplaque haemorrhage quantified using Hematoxylin and eosin staining (c, bar $1 \mathrm{~mm}$.) and glycophorine staining (d, bar $1 \mathrm{~mm}$.)

\section{ARWMC score}

Median ipsilateral ARMWC sum score was 2. Patients with an ipsilateral ARWMC sum score of $\leq 2(n=30)$ were compared to those with a score $>2(n=23)$. Patients with a high ARWMC 
sum score were significantly older $(72.5 \pm 6.46)$ compared to patients with a low score $(65.3 \pm$ 8.63, $p=.002$ ). Other baseline characteristics did not differ between the two groups, although estimated-packyears was identified as a potential confounder $(p=0.057$, supplemental table SII). Univariate analysis showed fewer macrophage staining in the high-ARWMC group (OR: $0.19 ; 95 \% \mathrm{Cl}: 0.05-0.75, p=.017$ ), which was no longer significant after adjustment for confounders in multivariate logistic regression (OR: $0.23295 \% \mathrm{Cl}: 0.052-10.02 p=.053$ ), see supplemental table SIII.

\section{Postoperative course}

The median follow-up period was 3.0 years (IQR: 2.9-3.2). Of the 53 included patients two developed perioperative ( $<30$ days) stroke, one ischaemic and one haemorrhagic, both were IPH-positive. No additional major perioperative events (MI or death) occurred in both IPHpositive or IPH-negative patients. In six additional patients a major postoperative arterial disease complication (nonfatal stroke, nonfatal Ml, vascular death) occurred during followup: two developed stroke (one ischaemic and one haemorrhagic); three suffered from MI and one additional patient died of other cardiovascular origin (sudden cardiac death). All but one of these patients were IPH-positive; of the IPH-negative patients one patient had MI, no $\mathrm{IPH}$-negative patients suffered from stroke or died of arterial disease complication during follow-up period.

\section{Discussion}

This study investigated the presence of recent silent ischaemic lesions in patients with IPH compared to those without IPH. We demonstrated that carotid IPH is associated with presence of MR-DWI ischaemic brain lesions in the waiting period between symptom onset and revascularization, identifying IPH as the potential pathological substrate for such lesions. This finding is in agreement with imaging studies on carotid plaque characteristics that show that intraplaque haemorrhage is an independent risk factor for future stroke or TIA in patients with carotid artery atherosclerosis ${ }^{16,17}$. This study, relating histological data to the development of silent ischaemic lesions, provides unique insight in natural course and pathology up until revascularization. The results suggest that revascularization procedures of patients with IPH should be prioritized over patients without IPH in order to avert the risk of recurrent ischaemia. It should be noted that this also applies in case of a pre- or in hospital delay of $>14$ days after the index event. Moreover, a broad consensus on early versus expedited treatment is still lacking ${ }^{6,18-20}$. This study contributes to the ongoing discussion on this matter by stressing the importance of eliminating an unstable plaque prior to any (clinical or subclinical) recurrent event. Although IPH cannot specifically identify all patients at risk for recurrent ischaemic events (silent or symptomatic) as IPH-rate is higher than DWI-rate, it can help to prioritize those that may benefit more from a rapid revascularization procedure. A more tailored therapy for symptomatic patients based on amongst others plaque imaging may be a solution for risk reduction of individual patients. 
One other clinical study showed that patients with symptomatic carotid stenosis with IPH are more likely to develop recurrent cerebrovascular events in the waiting period until revascularization ${ }^{21}$. This study did not use histological data but MR T1-weighted 3-dimensional gradient echo sequence to demonstrate IPH. Reliable methods for preoperative assessment of IPH on imaging are essential for clinical applicability. Over the years, several studies investigating IPH-imaging on MR have been performed using various sequences and protocols ${ }^{22,23}$. More recent articles propose magnetization-prepared rapid acquisition gradientecho (MPRAGE) as one of the most promising for sequence identification of IPH, with high sensitivity, specificity and $\mathrm{K}$-values when validated with histological data ${ }^{24}$. However, reliability of MR imaging of IPH in the plaque is still limited in case of smaller haemorrhages or coexisting calcifications ${ }^{24}$.

We found that presence of a large lipid core was negatively associated with DWI lesions whereas hypercholesterolemia, statin use and LDL-levels did not differ between groups. One might expect a lipid rich necrotic core to be positively associated with plaque instability and therefore recurrent events. Nonetheless, our results are in agreement with earlier research showing that large lipid core and macrophages are not associated with any vascular event, although no sub-analyses for cerebrovascular events specifically were performed in this study ${ }^{14}$. Another study comparing symptomatic to asymptomatic arteries within patients with unilateral symptomatic carotid stenosis found that IPH, but not a large lipid core, was more often seen on the symptomatic side ${ }^{25}$. Perhaps the presence of a large lipid core shows that plaque rupture has not yet occurred and therefore at this time does not characterize an acute risk for thrombo-embolisms.

Our study used MR-DWI in combination with ADC imaging as our primary outcome parameter. Presence of DWI lesions as a surrogate marker for cerebral ischaemia may become increasingly important, especially considering the evidence that DWI lesions are associated with a higher risk for recurrent cerebrovascular events ${ }^{7}$. In light of the continuously decreasing stroke-rate under improvements in procedural timing and medical therapy, stroke-rate alone is no longer sufficient for determination of therapeutic strategies. A surrogate marker for stroke is warranted and use of DWI as surrogate marker is both biological plausible and clinical relevant ${ }^{26}$. As the rate of recurrent cerebrovascular events in the period up until revascularization is as low as $1.6 \%^{27}$, use of DWI lesions (seen in $25 \%$ of our population) provides the opportunity to detect statistically significant differences in relatively small patient cohorts.

We did not find a difference in ARWMC score between IPH positive and IPH negative patients. Although one might hypothesize that plaque instability can contribute to development of age related white matter lesions via embolic events ${ }^{21}$, convincing evidence of a causal relation between the two was not demonstrated in earlier studies either ${ }^{28,29}$. A shared aetiology for atherosclerosis and white matter lesions may be more likely ${ }^{29}$.

\section{Limitations}

Intraplaque haemorrhage was determined histologically after the plaque was excided during CEA. Considering the long time interval between symptom onset and surgery, IPH was not 
necessarily already present at the moment of the index event. Relation to signs of IPH on preoperative imaging (MRI/duplex) may have improved the clinical applicability of our data. Additionally, we did not perform analyses to differentiate older (healed) IPH from acute IPH; although identification of healed IPH on histology is technically possible as shown in studies of coronary arteries ${ }^{30}$. Furthermore, DWI cannot be used to reliably estimate the age of an ischaemic lesion. Although ADC values can help to differentiate from older lesions by excluding T2-shinethrough, exact determination of the moment of onset of these lesions is impossible ${ }^{31}$ and we cannot rule out that the detected lesions were already present at hospital presentation.

In the context of clinical relevance, the impact of DWI-lesions on future cerebrovascular events would have been of interest. However, MR-DWI performed within few days after revascularization revealed that several patients developed (new) periprocedural DWI-lesions. Hence, any correlation between presence of DWI-lesions on preoperative imaging and future events is clouded by the carotid intervention shortly after and thus no statements can be made on the clinical impact of preprocedural silent ischaemic lesions.

No information was available on the presence or lack of IPH in other sites of the arterial tree, such as the aortic arch and the contralateral carotid artery. Possibly, IPH in the carotid artery was a sign of general inflammation and although a revascularization procedure of the ipsilateral carotid artery contributes to averting the risk of ipsilateral stroke it does not necessarily reduce the risk of any stroke. Additionally, we were not able to exclude non-arterial sources of embolism and any differences between patients in quality of medical intervention prior to baseline is lacking.

We are aware of the chance of type II statistical error or overfitting of our statistical model as a result of the small patient cohort. Patients of this study were included between 2004 and 2007 when, under current standards, substantial delay between hospital presentation and intervention was common practice. We do believe that the obtained knowledge is of surplus value as prioritizing patients may be challenging when multiple patients qualify for treatment at the same time. Also, patients with a late hospital-presentation may sometimes be postponed in favour of patients with more recent events. This study emphasizes the relevance of prompt acting even after passing of 14 days since index events. This small patient cohort also limits statistical comparison of the clinical course of IPH-positive versus IPH-negative as well as DWI-positive versus DWI-negative patients. Future research should focus on validation of the found results in a large cohort using both histological data as well as MRI-plaque data to compare to development of future cerebrovascular events.

\section{Future perspectives}

Symptomatic patients with a low risk of future events based on several clinical and radiological features are currently under investigation in the ECST-2 trial randomizing between best medical therapy alone or an additional revascularization procedure ${ }^{32}$. Assessment of IPH on plaque imaging can potentially help in stratification of these low-risk patients. Identification of histological IPH as a potential source of ischaemic brain lesions also suggests that assessment of plaque characteristics may be useful in decision making on optimal treatment 
strategy in asymptomatic carotid stenosis patients. Studies on imaging of carotid plaque IPH and follow-up in asymptomatic patients are needed to confirm this.

A meta-analysis including studies that performed MR plaque imaging on both symptomatic and asymptomatic patients demonstrated that patients with IPH have an annualized cerebrovascular event rate of $18 \%$ compared to $2 \%$ in those without $\mathrm{IPH}^{16}$. On the other hand, earlier research demonstrated that IPH is a common phenomenon in both symptomatic and asymptomatic patients ${ }^{33}$ suggesting that plaque complications such as IPH often heal without giving rise to symptoms and IPH alone may not be sufficient for risk stratification. We suggest that imminent clinical decision making-algorithms should include multiple risk factors as it has already been demonstrated that such models can reliably predict major cardiovascular events in patients with carotid artery disease ${ }^{34}$. These algorithms should take into account not only IPH but also other promising diagnostic tools such as brain imaging for detection of silent infarction and embolus-detection. Prospective observational studies are running that assess amongst others baseline MRI characteristics (both plaque and brain) to develop such a clinically applicable tool for risk stratification ${ }^{35}$.

\section{Conclusion}

In this study, we demonstrated that MR-DWI identified silent brain ischaemia in symptomatic patients undergoing CEA is associated with histologically apparent carotid plaque IPH. This qualifies IPH as a potential marker for identifying patients at risk for these ischaemic brain lesions. 


\section{References}

1. Michel JB, Virmani R, Arbustini E, Pasterkamp G. Intraplaque haemorrhages as the trigger of plaque vulnerability. Eur Heart J. 2011;32(16):1977-85.

2. Takaya N, Yuan C, Chu B, Saam T, Underbill H, Cai J, et al. Association between carotid plaque characteristics and subsequent ischemic cerebrovascular events: A prospective assessment with MRI Initial results. Stroke. 2006;37(3):818-23.

3. Turc G, Oppenheim C, Naggara O, Eker OF, Calvet D, Lacour JC, et al. Relationships between recent intraplaque hemorrhage and stroke risk factors in patients with carotid stenosis: The HIRISC study. Arterioscler Thromb Vasc Biol. 2012;32(2):492-9.

4. Vrijenhoek JEP, Den Ruijter HM, De Borst GJ, De Kleijn DPV, De Vries JPPM, Bots ML, et al. Sex is associated with the presence of atherosclerotic plaque hemorrhage and modifies the relation between plaque hemorrhage and cardiovascular outcome. Stroke. 2013;44(12):3318-23.

5. Naylor AR, Ricco JB, de Borst GJ, Debus S, de Haro J, Halliday A, et al. Management of Atherosclerotic Carotid and Vertebral Artery Disease: 2017 Clinical Practice Guidelines of the European Society for Vascular Surgery (ESVS). Eur J Vasc Endovasc Surg. 2018;55(1):3-81.

6. Meershoek AJA, de Borst GJ. Timing of carotid intervention. Br J Surg. 2018;105(10):1231-3.

7. Gensicke H, van der Worp HB, Nederkoorn PJ, Macdonald S, Gaines PA, Van Der Lugt A, et al. Ischemic brain lesions after carotid artery stenting increase future cerebrovascular risk. J Am Coll Cardiol. 2015 Feb;65(6):521-9.

8. Allen LM, Hasso AN, Handwerker J, Farid H. Sequence-specific MR Imaging Findings That Are Useful in Dating Ischemic Stroke. RadioGraphics. 2012;32(5):1285-97.

9. Bonati LHLH, Jongen LM, Haller S, Flach HZ, Dobson J, Nederkoorn PJ, et al. New ischaemic brain lesions on MRI after stenting or endarterectomy for symptomatic carotid stenosis: a substudy of the International Carotid Stenting Study (ICSS). Lancet Neurol. 2010 Apr;9(4):353-62.

10. Verhoeven BAN, Velema E, Schoneveld AH, De Vries JPPM, De Bruin P, Seldenrijk CA, et al. Atheroexpress: Differential atherosclerotic plaque expression of mRNA and protein in relation to cardiovascular events and patient characteristics. Rationale and design. Eur J Epidemiol. 2004;19(12):1127-33.

11. Ederle J, Dobson J, Featherstone RL, Bonati LH, van der Worp HB, de Borst GJ, et al. Carotid artery stenting compared with endarterectomy in patients with symptomatic carotid stenosis (International Carotid Stenting Study): an interim analysis of a randomised controlled trial. Lancet. 2010;375(9719):985-97.

12. Wahlund LO, Barkhof F, Fazekas F, Bronge L, Augustin M, Sjögren M, et al. A New Rating Scale for AgeRelated White Matter Changes. Stroke. 2001;32(6):1318-22.

13. Wesseling M, van Koeverden ID, van Lammeren GW, van der Laan SW, Haitjema S, de Vries JPPM, et al. Impaired kidney function is associated with intraplaque hemorrhage in patients undergoing carotid endarterectomy. Atherosclerosis. 2017;266:128-35.

14. Hellings WE, Peeters W, Moll FL, Piers S, Van Setten J, Van Der Spek PJ, et al. Composition of carotid atherosclerotic plaque is associated with cardiovascular outcome: A prognostic study. Circulation. 2010;121(17):1941-50.

15. Singh N, Moody AR, Zhang B, Kaminski I, Kapur K, Chiu S, et al. Age-Specific Sex Differences in Magnetic Resonance Imaging-Depicted Carotid Intraplaque Hemorrhage. Stroke. 2017;48(8):2129-35.

16. Saam T, Hetterich H, Hoffmann V, Yuan C, Dichgans M, Poppert H, et al. Meta-analysis and systematic review of the predictive value of carotid plaque hemorrhage on cerebrovascular events by magnetic resonance imaging. J Am Coll Cardiol. 2013;62(12):1081-91.

17. Gupta A, Baradaran H, Schweitzer AD, Kamel H, Pandya A, Delgado D, et al. Carotid plaque MRI and stroke risk: A systematic review and meta-analysis. Stroke. 2013;44(11):3071-7.

18. Tsantilas P, Kuehnl A, König T, Breitkreuz T, Kallmayer M, Knappich C, et al. Short Time Interval Between Neurologic Event and Carotid Surgery Is Not Associated With an Increased Procedural Risk. 2016;278390.

19. Sharpe R, Sayers RD, London NJM, Bown MJ, Mccarthy MJ, Nasim A, et al. Procedural Risk Following Carotid Endarterectomy in the Hyperacute Period after Onset of Symptoms. Eur J Vasc Endovasc Surg. 2013;46(5):519-24.

20. Strömberg S, Gelin J, Österberg T, Bergström GML, Karlström L, Österberg K. Very urgent carotid endarterectomy confers increased procedural risk. Stroke. 2012;43(5):1331-5.

21. Altaf N, MacSweeney ST, Gladman J, Auer DP. Carotid intraplaque hemorrhage predicts recurrent symptoms in patients with high-grade carotid stenosis. Stroke. 2007;38(5):1633-5.

22. Kerwin WS, Miller Z, Yuan C. Imaging of the high-risk carotid plaque : magnetic resonance imaging. Semin Vasc Surg. 2017;30(1):54-61.

23. McNally JS, Kim S, Mendes J, Hadley JR, Sakata A, De Havenon AH, et al. Magnetic Resonance Imaging Detection of Intraplaque Hemorrhage. Magn Reson Insights. 2017;10:1178623X1769415. 
24. Ota H, Yarnykh VL, Ferguson MS, Underhill HR, DeMarco JK, Zhu DC, et al. Carotid Intraplaque Hemorrhage Imaging at 3.0-T MR Imaging: Comparison of the Diagnostic Performance of Three T1weighted Sequences. Radiology. 2010;254(2):551-63.

25. Saam T, Cai J, Ma L, Cai Y-Q, Ferguson MS, Polissar NL, et al. Comparison of symptomatic and asymptomatic atherosclerotic carotid plaque features with in vivo MR imaging. Radiol. 2006;240(2).

26. Traenka C, Engelter ST, Brown MM, Dobson J, Frost C, Bonati LH. Silent brain infarcts on diffusionweighted imaging after carotid revascularisation: A surrogate outcome measure for procedural stroke? A systematic review and meta-analysis. Eur Stroke J. 2019;

27. Shahidi S, Owen-Falkenberg A, Gottschalksen B, Ellemann K. Risk of early recurrent stroke in symptomatic carotid stenosis after best medical therapy and before endarterectomy. Int J Stroke. 2016;11(1):41-51.

28. Potter GM, Doubal FN, Jackson CA, Sudlow CLM, Dennis MS, Wardlaw JM. Lack of association of white matter lesions with ipsilateral carotid artery stenosis. Cerebrovasc Dis. 2012;33(4):378-84.

29. van den Bouwhuijsen QJA, Vernooij MW, Verhaaren BFJ, Vrooman HA, Niessen WJ, Krestin GP, et al. Carotid Plaque Morphology and Ischemic Vascular Brain Disease on MRI. Am J Neuroradiol. 2017;38(9):1776-82.

30. Burke AP, Kolodgie FD, Farb A, Weber DK, Malcom GT, Smialek J, et al. Healed Plaque Ruptures and Sudden Coronary Death. Circulation. 2001;103(7):934 LP-940.

31. Abbott AL, Silvestrini M, Topakian R, Golledge J, Brunser AM, de Borst GJ, et al. Optimizing the definitions of stroke, transient ischemic attack, and infarction for research and application in clinical practice. Front Neurol. 2017;8(OCT):1-14.

32. Featherstone RL, Brown MM. The second european carotid surgery trial. Endovasc today. 2012;(October):75-7.

33. Bassiouny HS, Davis H, Massawa N, Gewertz BL, Glagov S, Zarins CK. Critical carotid stenoses: Morphologic and chemical similarity between symptomatic and asymptomatic plaques. J Vasc Surg. 1989;9(2):202-12.

34. Khanna NN, Jamthikar AD, Araki T, Gupta D, Piga M, Saba L, et al. Nonlinear model for the carotid artery disease 10-year risk prediction by fusing conventional cardiovascular factors to carotid ultrasound image phenotypes: A Japanese diabetes cohort study. Echocardiography. 2019;36(2):345-61.

35. TAXINOMISIS project. 2018. p. https://taxinomisis-project.eu/. 


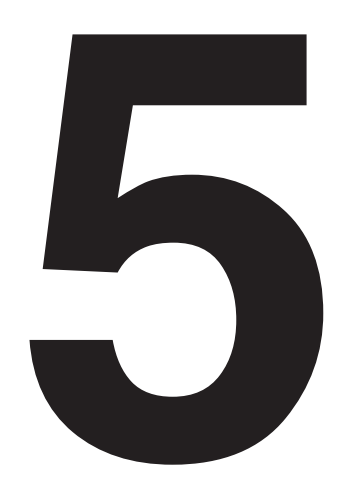

\section{Cerebral small vessel disease in standard preoperative imaging reports is independently associated with increased risk of cardiovascular death following carotid endarterectomy}

N Timmerman, ML Rots, ID van Koeverden, S Haitjema, CJHCM van Laarhoven, AM Vuurens, HM den Ruijter, G Pasterkamp, LJ Kappelle, DPV de Kleijn, GJ de Borst Eur J Vasc Endovasc Surg 2020; in press 


\section{Abstract}

Background. Cerebral white-matter lesions (WMLs) and lacunar infarcts are surrogates of cerebral small vessel disease (SVD). WML severity as determined by trained radiologists predicts postoperative stroke or death in patients undergoing carotid endarterectomy (CEA). It is unknown if routine preoperative brain imaging reports as part of standard clinical practice also predicts short- and long-term risk of stroke and death after CEA.

Methods. Consecutive patients from the Athero-Express biobank study that underwent CEA for symptomatic high degree stenosis between March 2002 and November 2014 were included. Preoperative brain imaging (CT- or MRI) reports were reviewed for reporting of SVD, defined as WMLs or any lacunar infarcts. The primary outcome was defined as any stroke or any cardiovascular death during 3-year follow-up. The secondary outcome was defined as the 30-day perioperative risk of stroke or cardiovascular death.

Results. 1038 patients were included (34\% women), of whom $659(63.5 \%)$ had CT images and $379(36.5 \%)$ had MRI available. Of all patients, 697 (67\%) patients had SVD reported by radiologists. Patients with SVD had a higher 3-year risk of cardiovascular death compared to those without (6.5\% vs. $2.1 \%$, adjusted HR 2.52 (95\% Cl: $1.12-5.67) ; p=.026)$ but no association was observed for the 3 -year risk of stroke $(9.0 \%$ vs. $6.7 \%$, for patients with SVD versus those without, adjusted HR 1.24 (95\% Cl: 0.76-2.02); $p=.395)$. No differences in 30-day perioperative risk were observed for stroke $(4.4 \%$ vs. $2.9 \%$, for patients with versus those without SVD; adjusted HR 1.49 (95\% $\mathrm{Cl}: 0.73-3.05) ; p=.277)$, and for the combined stroke/cardiovascular death risk (4.4\% vs. 3.5\%, adjusted HR 1.20 (95\%Cl: 0.61-2.35); $p=.592)$.

Conclusions. Presence of SVD in preoperative brain imaging reports can serve as a predictor for the 3-year risk of cardiovascular death in symptomatic patients undergoing CEA, but does not predict perioperative or long-term risk of stroke. 


\section{Introduction}

Carotid endarterectomy (CEA) is the preferred treatment for high degree carotid stenosis in symptomatic patients although procedural stroke may limit the benefit of the intervention. ${ }^{1}$ Patient-specific predictors of high procedural- or long-term stroke risk can be used to optimize patient selection for revascularization and individualized medical treatment. ${ }^{2}$ The presence of cerebral white-matter lesions (WMLs) on preoperative computed tomography (CT) or magnetic resonance imaging (MRI) has been previously suggested as a predictor for perioperative adverse events following CEA. ${ }^{3}$

WMLs are a common finding on baseline CT- and MRI brain scans. These are closely associated with age and risk factors for atherosclerosis and have been independently associated with increased risk for dementia, first-ever stroke, recurrent stroke, poor functional stroke outcome, and cardiovascular mortality. ${ }^{4,5}$ The pathogenesis of WMLs is multifactorial and suggested to be caused by small artery atherosclerosis leading to chronic cerebral hypoperfusion and white matter ischemia. ${ }^{6,7}$ Previous studies in symptomatic CEA patients suggested an increased peri- and postoperative stroke- and death risk for patients with more extensive WMLs. ${ }^{3,8,9}$ The association between WMLs and an increased risk for stroke may be related to impaired cerebral haemodynamics due to a reduced vessel density, impaired vessel autoregulation or a reduced baseline cerebral blood flow. ${ }^{6,10}$ Therefore, it can be hypothesized that a brain with more than age-appropriate WMLs may be more vulnerable to develop subsequent ischemic infarct when challenged by acute thromboembolic or haemodynamic events.

While previous studies investigated the predictive value of specific (semi-)quantitative WML severity-scores for adverse events in patients undergoing $\mathrm{CEA}^{3,8,9}$, thus far no studies have evaluated the clinical relevance of cerebral small vessel disease (SVD) as reported in daily practice. The current study aimed to assess the clinical relevance of baseline SVD in standard preoperative brain imaging reports in relation to future adverse cardiovascular events following CEA. We hypothesized that the presence of SVD is associated with increased stroke or cardiovascular death risk in symptomatic patients undergoing CEA.

\section{Methods}

\section{Athero-Express}

All patients were recruited from the Athero-Express biobank study, of which the design has been published elsewhere. ${ }^{11,12}$ This ongoing prospective cohort study includes consecutive patients with carotid artery stenosis undergoing CEA in two large tertiary referral hospitals in the Netherlands, the University Medical Centre in Utrecht and the St. Antonius Hospital in Nieuwegein. Patient characteristics, including cardiovascular risk factors, history of cardiovascular disease, history of peripheral artery disease and medication use were obtained from standardized questionnaires and patient records at time of inclusion. Patients included in the study were symptomatic (stroke, hemispheric transient ischemic attacks (TIA) 
or monocular blindness $<6$ months prior to hospital presentation) with a high-grade carotid artery stenosis of $50-99 \%$. The indication for CEA was reviewed by a multidisciplinary vascular team and was based on local and international guidelines. ${ }^{13-17}$ Follow-up data were obtained for secondary cardiovascular and cerebrovascular events during the 3-year postoperative period. The study protocol was approved by the medical ethics committees of both hospitals and patients provided written informed consent before participation in the Athero-Express study. All consecutive patients included in the Athero-Express and undergoing CEA for symptomatic carotid artery stenosis between January 2002 and November 2014 were included in the present analysis. Patients underwent routine preoperative brain CT- or MRI and/or CT- or MR-angiography, in concordance with international guidelines. ${ }^{13-17}$ Patients were excluded for analysis when baseline brain imaging reports were missing or incomplete (i.e. when only a concise imaging report was available from a referring center) or in case of lost to follow-up.

\section{Definition small vessel disease}

The routine preoperative brain $\mathrm{CT}$ and/or MRI reports were retrospectively analysed for the presence or absence of SVD. SVD was defined as any reported WML (or a synonym, see supplemental table S1) or any old lacunar infarct. This definition was chosen due to the fact that both modalities have the same aetiology and both have been proven to predict future stroke in the general population. ${ }^{4,7}$ To assess the accuracy of reporting SVD in standard clinical imaging reports, a subset of 30 scans were randomly selected from the total cohort for validation. Radiology images were meticulously evaluated for WML and lacunar infarct by a dedicated observer (MR) who was blinded for the standard clinical reports. Reliability (kappa-values) and proportion of agreement were calculated between reported WML and lacunar infarct in standard clinical imaging reports and those identified by re-evaluation of the radiology images of the blinded observer (MR).

\section{Follow-up and outcome}

The primary outcome was defined as any stroke or any cardiovascular death during 3-year follow-up. Any stroke includes nonfatal ischemic stroke, nonfatal haemorrhagic stroke, fatal ischemic stroke and fatal haemorrhagic stroke. Any cardiovascular death includes death of presumed vascular origin such as fatal stroke, fatal myocardial infarction and sudden death, fatal cardiac failure, fatal aneurysm rupture. Secondary outcomes were all-cause death during 3-year follow-up and the composite endpoint of any stroke or any cardiovascular death during the 30-day perioperative period. The follow-up protocol was identical to the one previously described. ${ }^{11,12}$ Perioperative assessment was structurally done by a neurologist and/or vascular surgeon. Patient medical records were checked for perioperative adverse events. Patients were contacted annually for three years after CEA through standardized questionnaires asking whether they had experienced an adverse cardiovascular event or if they had been hospitalized. Questionnaires were verified with medical records; when a questionnaire is answered positively, additional data (medical records, electrocardiograms, laboratory and imaging studies) are retrieved and assessed for the occurrence of an outcome event. In case of non-response, the general practitioner was consulted. All available data 
to establish an outcome event were reviewed by two independent experts. In case of a disagreement, a third expert was consulted and outcome was discussed until agreement was reached.

\section{Statistical analysis}

Baseline characteristics and survival-free and stroke-free rates were compared between patients with reported SVD and patients without reported SVD. For continuous baseline variables, the independent samples t-test was used for normally distributed variables and Mann Whitney $U$ test for non-normally distributed variables. Categorical baseline variables were tested by the chi-squared test for equal distribution among both groups. The cumulative incidences of the outcome measures were estimated using a Kaplan-Meier analysis. To investigate the association between reported SVD and outcomes, Cox proportional-hazards regression modelling was used for the univariate and multivariable analysis. For baseline characteristics with an association of $\mathrm{p}<.10$ with reported SVD, an association with outcome measures was also checked using Cox proportional-hazards regression modelling. Whenever statistical significance of $p<.10$ with the outcome of interest was also found, the baseline variable was added as a covariate in the multivariable models (supplemental table S2). Since contralateral carotid stenosis is considered as a strong predictor for future stroke, we included this as covariate in the multivariable analysis (full models are displayed in supplemental table S2). Missing data of covariates were imputed with multiple imputation to enable multivariable analysis (supplemental table S3). To quantify follow-up completeness in this study, the followup index (FUI) was calculated as described previously ${ }^{18}$ and compared between both study groups. All statistical analyses were performed with IBM SPSS statistics version 25.0 (SPSS Inc, Chicago, IL). A p-value $<.05$ was considered as statistically significant.

\section{Results}

Patient selection for the current study is displayed in figure 1. Of the 1544 patients who met inclusion criteria, 1038 patients had available preoperative brain imaging reports and were included for analysis. Of those, 659 (63.5\%) had a CT-scan and 379 (36.5\%) had an MRI-scan preoperatively.

\section{Imaging characteristics}

Various CT- and MRI-scanners and scanning protocols were used. Slice thickness of CT scanning varied from 3 to $6 \mathrm{~mm}$. MRI T2- fluid-attenuated inversion-recovery (FLAIR) sequences were most often used, predominantly with field strengths of 1.5 Tesla (and few of 3.0 Tesla), and varying slice thickness from 4 to $6 \mathrm{~mm}$. Reasons for missing brain imaging (reports) included that only CT- or MRI angiography was performed (specifically in patients presenting with ocular symptoms) or referral from other hospitals from which imaging reports were not included in the referral data. Differences in baseline characteristics between included patients and patients with missing brain imaging or reports are displayed in supplemental table S4. Reliability of SVD identification from imaging reports compared with the SVD scoring of the radiology images by blinded observer was good (к $0.74 \pm 0.14$, see supplemental table S5). 


\section{Presence of SVD}

In the total cohort of 1038 patients $34 \%$ were women, $18 \%$ had presented with transient or permanent retinal ischemia, 35\% with stroke and 43\% with TIA (table 1). SVD was reported in 697 patients (67\%). Of patients with SVD, 243 (35\%) were female versus 108 (32\%) without SVD ( $p=.307)$. SVD was reported in $51 \%$ of patients $<60$ years, $61 \%$ of patients between $60-70$ years, $72 \%$ of patients between $70-80$ years and $82 \%$ of patients $>80$ years old $(p<.001)$. Detailed characteristics of SVD lesions are displayed in figure 1. Of all patients with reported SVD, 147/697 (21\%) had WMLs and lacunar infarct, 431/697 (62\%) had only WML and 119/691 (17\%) had only lacunar infarct. SVD was located ipsilateral to the operated artery in 111/697 patients (16\%), in 56/697 (8\%) contralateral and bilateral in 235/697 patients (34\%), whereas in 295/697 patients (42\%) the location was not specified in the imaging report. Baseline characteristics of patients associated with SVD are shown in table 1. Patients with SVD were older, more frequently hypertensive and diabetic, had decreased renal function and more frequently had a history of peripheral artery disease.

\section{Association between SVD and stroke or death}

The FUI for patients with SVD and those without were equal (mean FUI $0.95 \pm 0.15$ versus $0.97 \pm 0.11$ respectively, $p=.976$ ) indicating complete follow-up information for both study groups. The 3-year risk of the composite endpoint for stroke and cardiovascular death was similar for patients with and patients without SVD (12.6\% vs $8.2 \%$ respectively, adjusted hazard ratio (HR) 1.36 (95\% confidence interval (Cl): $0.88-2.10) ; p=.172$; table 2 , figure $2 \mathrm{~A}$ ) as was the risk of any stroke $(9.0 \%$ vs. $6.7 \%$, adjusted HR 1.24 (95\% Cl: 0.76-2.02); $p=.395$; table 2, figure 2B). The 3-year risk of cardiovascular death was significantly higher in patients with reported SVD compared to patients without $(6.5 \%$ vs. $2.1 \%$, adjusted HR 2.52 (95\% Cl: 1.12-5.67); $p=.026$; table 2, figure $2 \mathrm{C}$ ). The 3-year risk of all-cause death did not significantly differ between patients with reported SVD compared to those without (13.9\% vs. $8.2 \%$, adjusted HR 1.26 (95\%Cl: 0.82-1.94); $p=.298$; supplemental table S6). For the 30-day perioperative period, the combined stroke or cardiovascular death risks were similar for patients with and patients without SVD (4.4\% vs. 3.5\% respectively, with adjusted HR $1.2095 \% \mathrm{Cl}$ : $0.61-2.35) ; p=.592$; table 3, figure 3A). Also, no difference in 30-day perioperative stroke risk was observed (4.4\% vs. $2.9 \%$; adjusted HR 1.49 (95\% Cl: 0.73-3.05); $p=.277$; table 3, figure 3B).

\section{Sub-analyses}

Separate analyses for the CT- and MRI-cohort were performed and added to the supplemental materials (supplemental tables S7a-c). For those that had CT, 381 out of $659(57.8 \%)$ had SVD and for those that had MRI 316 out of 379 patients (83.3\%) had SVD. Similar trends in differences in baseline characteristics were observed for CT and MRI- subgroups. The independent association between SVD and 3-year risk of cardiovascular death was confirmed in the CT-subgroup analysis (absolute incidences of $7.9 \%$ versus $2.5 \%$, respectively with adjusted $\mathrm{HR} 2.56$ (95\% Cl:1.09-6.00); $p=0.031$ ) but not in the MRI subgroup (absolute incidences of $4.7 \%$ versus $0.0 \%$ in patients with SVD versus those without). However, univariate analysis for MRI was inaccurate (unrealistic HR and wide $\mathrm{Cl}$ ) and multivariable analyses could not be performed, both because of limited power. Because the location of SVD was not specified in the reports in a large proportion of the patients $(42 \%)$, sub-analysis based on different location (ipsilateral versus contralateral versus bilateral) could not be performed. 
Table 1 Baseline characteristics of symptomatic CEA patients compared between those with reported cerebral small vessel disease and those without.

\begin{tabular}{|c|c|c|c|}
\hline Baseline characteristics & $\begin{array}{l}\text { No small vessel } \\
\text { disease } \\
(n=341)\end{array}$ & $\begin{array}{l}\text { Small vessel } \\
\text { disease } \\
(n=697)\end{array}$ & $p$ value \\
\hline Age, years (mean [SD]) & $67.2[9.3]$ & $71.7[9.3]$ & $<.001^{b}$ \\
\hline Women & $108(31.7)$ & $243(34.9)$ & .307 \\
\hline Hypertension & $231(70.2)$ & $530(79.1)$ & $.002^{b}$ \\
\hline Diabetes & $65(19.1)$ & $189(27.1)$ & $.005^{\mathrm{b}}$ \\
\hline BMI, kg/m² (mean [SD]) & $26.5[4.1]$ & $26.2[4.0]$ & .382 \\
\hline Hypercholesterolemia & $205(66.3)$ & $420(67.1)$ & .819 \\
\hline Current smoker & $125(37.8)$ & $213(31.2)$ & $.039^{b}$ \\
\hline Packyears of smoking & & & .611 \\
\hline$\Phi 0$ & $50(16.9)$ & $116(19.9)$ & \\
\hline Ф 1-10 & $186(62.8)$ & $355(60.8)$ & \\
\hline Ф 10-20 & $40(13.5)$ & $84(14.4)$ & \\
\hline Ф 20-30 & $16(5.4)$ & $21(3.6)$ & \\
\hline Ф $30-40$ & $4(1.4)$ & $8(1.4)$ & \\
\hline History of CAD & $90(26.4)$ & $213(30.6)$ & .157 \\
\hline History of PAD & $40(11.8)$ & $136(19.6)$ & $.002^{b}$ \\
\hline eGFR, ml/min/1/1.73m² (mean, [SD]) & $76.2[19.7]$ & $69.9[20.9]$ & $<.001^{b}$ \\
\hline Ipsilateral carotid stenosis & & & .241 \\
\hline Ф $50-70 \%$ & $43(12.6)$ & $71(10.2)$ & \\
\hline Ф $70-99 \%$ & $298(87.4)$ & $626(89.8)$ & \\
\hline Contralateral carotid stenosis $(50-100 \%)$ & $127(42.5)$ & $248(39.2)$ & .347 \\
\hline Presenting cerebrovascular event & & & $.079^{b}$ \\
\hline$\Phi$ stroke & $115(33.7)$ & $249(35.7)$ & \\
\hline Ф TIA & $141(41.3)$ & $308(44.2)$ & \\
\hline Ф ocular & $76(22.3)$ & $112(16.1)$ & \\
\hline$\Phi$ unclear & $9(2.6)$ & $28(4.0)$ & \\
\hline History of any stroke & $133(39.0)$ & $308(44.2)$ & .112 \\
\hline Statin use & $263(77.4)$ & $525(75.5)$ & .520 \\
\hline Antiplatelet drug use & $294(86.7)$ & $611(88.0)$ & .547 \\
\hline Oral anticoagulant use & $39(11.5)$ & $74(10.6)$ & .690 \\
\hline
\end{tabular}

Data are displayed as $\mathrm{n}(\%$ of total) unless otherwise indicated. Numbers in bold indicate $\mathrm{p}<.05$.

${ }^{b}$ Baseline characteristic showing $\mathrm{p}<.10$ association with reported cerebral small vessel disease $\mathrm{BMI}=$ Body mass index; $\mathrm{CAD}=$ coronary artery disease; $\mathrm{PAD}=$ peripheral artery disease; eGFR = estimated glomerular filtration rate; TIA = transient ischaemic attack; $S D=$ standard deviation.

When multiple cerebrovascular symptoms occurred in the six months prior to the operation, the most serious symptom counts in the following order: stroke $>$ TIA $>$ ocular. Ocular symptoms include transient or permanent retinal ischaemia.

History of any stroke includes ipsilateral or contralateral stroke.

Antiplatelet drug use includes the use of aspirin, dipyridamole or any ADP-inhibitor. 


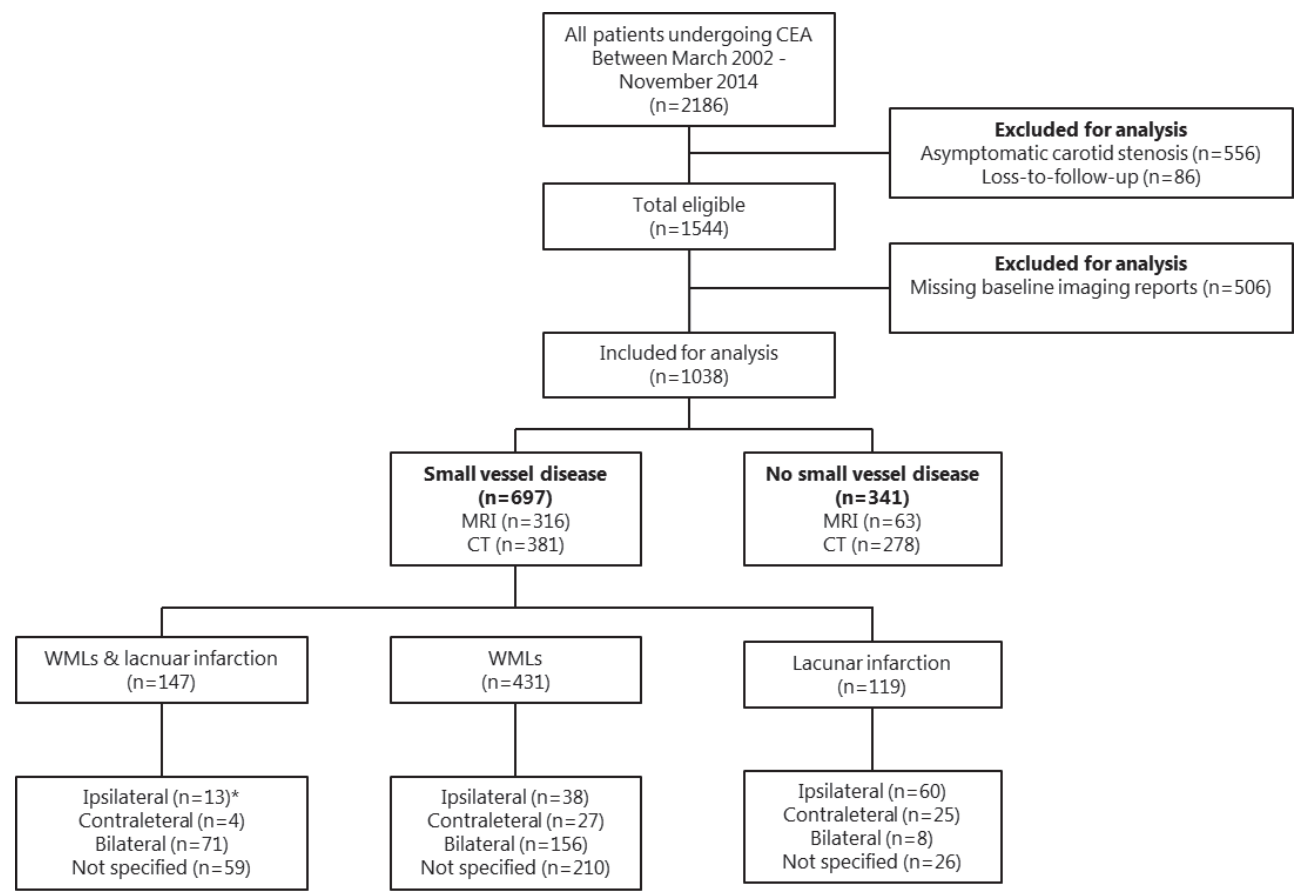

Figure 1 Flowchart of included patients from the Athero-Express Biobank and type of SVD lesions.

Numbers in brackets indicate numbers of patients. Every patient can only belong in one category (numbers are mutually exclusive). ${ }^{*}$ The location relates to any lesion including WML or lacunar infarct or both.

Table 2. Cox-proportional hazards analysis of 3-year risks for secondary adverse events after CEA.

\begin{tabular}{|c|c|c|c|c|c|c|}
\hline \multirow[b]{2}{*}{$\begin{array}{l}\text { 3-year follow-up } \\
\text { outcome measure }\end{array}$} & \multirow[b]{2}{*}{$\begin{array}{l}\text { No small } \\
\text { vessel disease }\end{array}$} & \multirow[b]{2}{*}{$\begin{array}{l}\text { Small vessel } \\
\text { disease }\end{array}$} & \multicolumn{2}{|c|}{ Univariate analysis } & \multicolumn{2}{|c|}{ Multivariable analysis } \\
\hline & & & $\mathrm{HR}[95 \% \mathrm{Cl}]$ & p-value & $\mathrm{HR}[95 \% \mathrm{Cl}]$ & $p$ value \\
\hline $\begin{array}{l}\text { Any stroke or } \\
\text { cardiovascular } \\
\text { death }^{\text {a }}\end{array}$ & 28/341 (8.2\%) & $88 / 697$ (12.6\%) & $1.59[1.04-2.43]$ & .033 & $1.36[0.88-2.10]^{a}$ & .172 \\
\hline Any stroke ${ }^{b}$ & 23/341 (6.7\%) & $63 / 697$ (9.0\%) & $1.38[0.85-2.22]$ & .190 & $1.24[0.76-2.02]^{\mathrm{b}}$ & .395 \\
\hline $\begin{array}{l}\text { Cardiovascular } \\
\text { death }{ }^{c}\end{array}$ & $7 / 341$ (2.1\%) & $45 / 697(6.5 \%)$ & 3.29 [1.48-7.30] & .003 & $2.52[1.12-5.67]^{c}$ & .026 \\
\hline
\end{tabular}

$H R$, hazard ratio. $H R>1.0$ indicates an increased risk for patients with cerebral small vessel disease compared to patients without. Numbers in bold indicate $p<.05$.

${ }^{a}$ Covariates included in multivariable model of the composite endpoint any stroke or cardiovascular death were age, cerebrovascular symptoms, diabetes, history of PAD, eGFR and contralateral stenosis (50-100\%).

${ }^{\mathrm{b}}$ Covariates included in multivariable model of any stroke were age, diabetes and contralateral stenosis (50-100\%).

${ }^{c}$ Covariates included in multivariable model of cardiovascular death were age, history of PAD, eGFR and contralateral stenosis (50-100\%). 


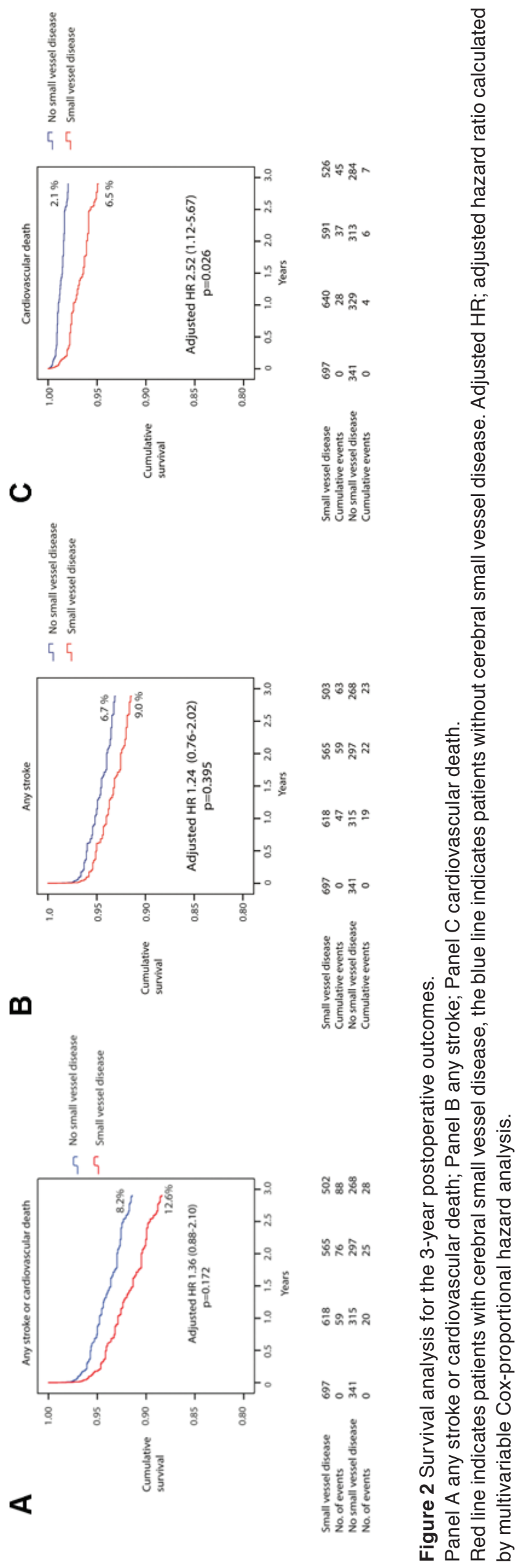


Table 3 Cox-proportional hazards analysis of 30-day perioperative risks for secondary adverse events after CEA.

\begin{tabular}{lllllll}
\hline & & \multicolumn{3}{c}{ Univariate analysis } & \multicolumn{2}{c}{ Multivariable analysis } \\
$\begin{array}{l}\text { Perioperative } \\
\text { outcome } \\
\text { measure }\end{array}$ & $\begin{array}{l}\text { No small } \\
\text { vessel } \\
\text { disease }\end{array}$ & $\begin{array}{l}\text { Small vessel } \\
\text { disease }\end{array}$ & HR [95\% Cl] & p-value & HR [95\%Cl] & p-value \\
\hline $\begin{array}{l}\text { Any stroke or } \\
\text { cardiovascular } \\
\text { death }^{a}\end{array}$ & $12 / 341(3.5 \%)$ & $31 / 697(4.4 \%)$ & $1.27[0.65-2.47]$ & .484 & $1.20[0.61-2.35]$ & .592 \\
Any stroke $^{\text {b }}$ & $10 / 341(2.9 \%)$ & $31 / 697(4.4 \%)$ & $1.52[0.75-3.11]$ & .248 & $1.49[0.73-3.05]$ & .277 \\
\hline
\end{tabular}

$H R$, hazard ratio. $\mathrm{HR}>1.0$ indicates an increased risk for patients with cerebral small vessel disease compared to patients without. Numbers in bold indicate $p<.05$.

a Covariates included in multivariable model of any stroke or cardiovascular death were cerebrovascular symptoms, diabetes and contralateral stenosis (50-100\%).

${ }^{b}$ Covariates included in multivariable model of any stroke were diabetes and contralateral stenosis (50-100\%)

A

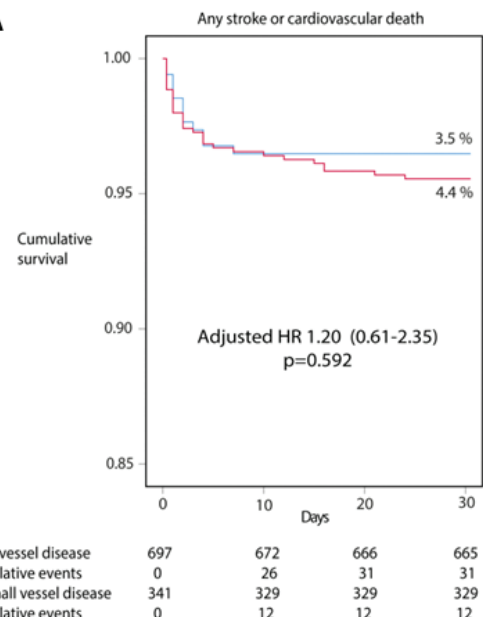

B

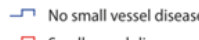
$\neg$ No small vessel disease

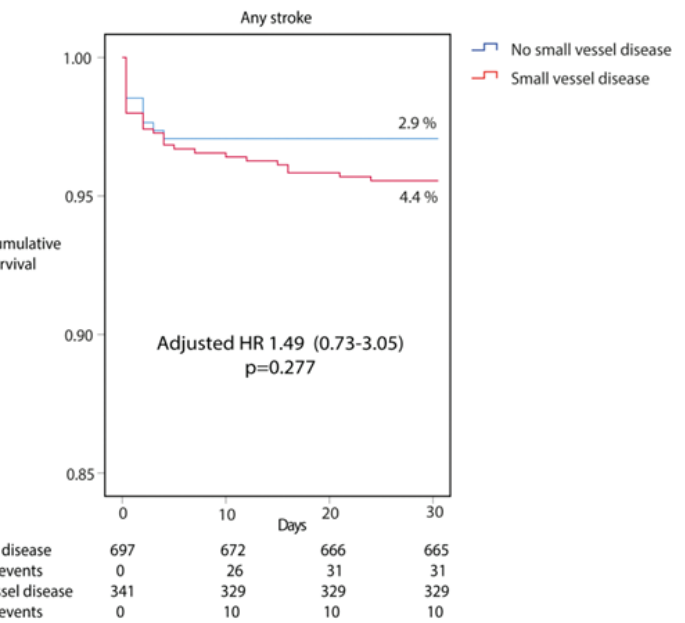

Figure 3 Survival analysis for the 30-day perioperative outcomes Panel A any stroke or cardiovascular death; Panel B any stroke. Red line indicates patients with cerebral small vessel disease, the blue line indicates patients without cerebral small vessel disease. Adjusted HR; adjusted hazard ratio calculated by multivariable Cox-proportional hazard analysis.

\section{Discussion}

This study examined the clinical relevance of reported SVD in preoperative routine clinical brain imaging reports for perioperative and long-term adverse cardiovascular events in symptomatic patients undergoing CEA. Preoperative SVD was reported in two thirds of patients undergoing CEA and was associated with a 2.5-fold increased 3-year risk of postoperative cardiovascular death, independent of known cardiovascular risk factors. Our results indicate that reported SVD can identify patients with poor survival and accordingly a potentially lower long-term benefit of CEA. Yet, as reported SVD was not associated with procedural or postoperative 
stroke-risk, SVD should not be used to weigh up the indication for carotid intervention in symptomatic patients.

In line with our results, the International Carotid Stenting Study (ICSS) revealed no association between higher scores of age-related white matter changes (ARWMC) and 30-day perioperative stroke risk in CEA patients. ${ }^{9}$ On the contrary, the North American Symptomatic Endarterectomy Trial (NASCET) observed increased 30-day combined stroke or death risks in patients with widespread WMLs compared to those without (13.9\% versus $5.3 \%) .{ }^{3}$ It is worth noting that the overall 30-day perioperative stroke or death rate in the NASCET $(6.7 \%)$ was higher than the CEA cohort of the ICSS (3.4\%) and our cohort (4.1\%). These differences could be attributed to the improved surgical techniques and novel insights in anaesthesiologic management. ${ }^{19}$ Moreover, it has been shown that concomitantly with improved cardiovascular prevention therapy, atherosclerotic plaques have stabilized over time. ${ }^{20}$ It could be hypothesized that due to plaque stabilization the occurrence of procedural embolisms has been reduced, resulting in lower stroke rates. Additionally, perioperative stroke is multifactorial, varying from thrombosis or thrombotic occlusion of the operated carotid artery, hyperperfusion syndrome, carotid-embolism or cardio-embolism and intraoperative hypoperfusion. ${ }^{21}$ Therefore SVD may not be one of the main predictors for these heterogeneous types of stroke with various pathophysiological mechanisms.

Regarding the long-term postoperative risk of stroke, the only available evidence results from the aforementioned NASCET that observed an increased 3-year postoperative stroke risk in patients with more extensive WMLs. ${ }^{3}$ Of note, increased long-term stroke risk was also observed in the medically treated group of this study. As discussed above, the NASCET cohort does not reflect the current stroke risk in patients undergoing CEA. Although WMLs predict recurrent stroke in patients with ischemic stroke in general ${ }^{5}$, these results cannot be directly extrapolated to patients with carotid stenosis because the risks of future cardiovascular events and survival varies between different aetiologies of ischemic stroke. ${ }^{22,23}$ Furthermore, prevalence of WMLs in our study was higher than previously described ${ }^{3}$, likely as a result of more sensitive scanning techniques. Earlier studies may have selected patients with more severe WML, resulting in more pronounced differences between groups with, as a consequence, a stronger association between WML and short- and long-term outcome events. We cannot exclude that preoperative reported SVD may have a small predictive value for stroke, but larger cohort studies, including assessment of WML load and localization, would be needed to confirm this.

Our finding that increased postoperative long-term risk of cardiovascular death in symptomatic patients undergoing CEA is associated with reported SVD is in line with a previous small study ${ }^{8}$, which showed that CEA patients (predominantly symptomatic) with any quantified WML on preoperative brain CT had an increased 5-year risk of mortality (predominantly of cardiovascular origin). We now show that, also based on standard brain imaging reports, SVD can be used as predictor for cardiovascular death. Preoperative SVD was associated with all-cause death in univariate analysis, yet this effect was diminished in multivariable analysis implying that SVD is not independently associated with all-cause mortality. This discrepancy with the previous study ${ }^{8}$ could be 
attributed to the shorter follow-up period or could be explained by the fact that the previous study did not fully adjust for confounders due to small sample size. The overall increased risk of cardiovascular death can be explained by the concept of systemic SVD due to generalized atherosclerotic disease, resulting in end organ manifestations such as kidney failure or cardiomyopathy. ${ }^{24,25}$ Furthermore, it is suggested that endothelial dysfunction in both cerebral arteries and systemic arteries is increased in patients with more severe WMLs. ${ }^{26}$ A shared aetiology between SVD and cardiovascular death specifically is supported by the fact that no differences in all-cause death were found between patients with and those without SVD. Reliable estimates for long-term survival rates in patients qualifying for CEA are important to determine the absolute net-benefit of the procedure. As long-term recurrent stroke risk has decreased for symptomatic patients with carotid artery disease because of improved preventive medical strategies, the number-neededto-treat of CEA may be higher than previously determined. When patients have a shorter life-expectancy due to severe systemic atherosclerotic disease, the duration of benefit of a revascularization procedure will be shorter. When assessment of SVD becomes part of standard clinical workup this can be used in future risk stratification algorithms that use multiple demographic, biological and imaging characteristics for decision making on optimal treatment strategy. ${ }^{27}$

\section{Strengths and limitations}

This study has several limitations. First, both CT and MRI brain scan reports were used, while MRI is more sensitive in detection of small WMLs. ${ }^{28}$ This could explain the higher prevalence of SVD found in the MRI subgroup (83.3\%) compared with the CT subgroup (57.8\%). Subgroup analyses confirmed the association of SVD with cardiovascular death in the CT-subcohort, but in the MRI-subcohort this could not be determined because of the limited sample size and limited number of events. However, both CT and MRI are interchangeably used in routine clinical practice and this study addresses its clinical relevance. Furthermore, differences in event-rates between patients with and without SVD are similar in CT and MRI subgroups and thus type of imaging modality does not seem to have large impact on the results. Yet, MRI may have led to the detection of minor lesions that may have been missed with CT. Patients with more severe lesions may have a higher risk of generalized atherosclerotic disease and are therefore at higher risk of $\mathrm{CV}$ death. Detection of all (minor and major) lesions in MRI-patients may have diluted the effect on outcome in these patients. As MRI is increasingly being used in clinical setting for diagnosis of stroke, future studies of larger sample size, also focusing on the difference between minor and major lesions, are needed to elaborate the association of preoperative SVD based on MRI and cardiovascular death. Second, CT and MRI imaging was performed with different scanners and different protocols due to the broad study time. This study therefore reflects routine clinical care. Third, in our analysis no distinction was made between ipsi- and contralateral or between mild and severe SVD. One can imagine that ipsilateral SVD is more likely to be attributable to thromboembolic events due to ipsilateral carotid stenosis, whereas bilateral WML may be an expression of generalized atherosclerotic disease. Semi-quantitative methods for assessment of severity of WML are widely available 
and generally well-known, but are not consistently used in standard imaging reports. By pooling all patients with any reported WML we might have underestimated the correlation between SVD and outcome. Yet, previous studies that did investigate the correlation between (semi-) quantitative WML measures and outcome in patients undergoing CEA used trained radiologists for assessment of these study parameter which has the disadvantage of not being part of standard clinical practice. ${ }^{3,8,9}$ Fourth, we only included symptomatic patients whereas assessment of perioperative risk in asymptomatic patients may be even more relevant in the ongoing discussion on whether or not to perform revascularization procedures in these patients. Finally, stroke was less frequently observed for excluded patients compared to included patients (see supplemental table S4), mainly because imaging reports were not attached in the referral from other hospitals. Excluded patients from the present study could be considered as lower risk patients, which would have rather led to an overestimation of the association between SVD and stroke and would not have changed our conclusion.

Despite limitations, the present study includes a large CEA cohort reflecting current incidences of perioperative and postoperative adverse events. Additionally, the study design mimics daily practice in which clinicians evaluate the presence of SVD based on brain imaging reports constructed by radiologists through standardized protocols and not using non-standard quantification techniques. Future research should focus on how preoperative baseline imaging can be used for determining optimal treatment strategy. ${ }^{29}$ Such studies should also focus on the relation between WML and other promising imaging markers such as (silent) ischemic brain lesions, which have been associated with poor outcome and WMLs. ${ }^{30,31}$ Studies should report predictive value of both ipsi- and contralateral cerebral lesions, should investigate methods for easy identification and quantification of lesions, and should stratify symptomatic from asymptomatic patients in their analysis.

\section{Conclusion}

In clinical practice, SVD in standard preoperative brain imaging reports is independently associated with general increased risk of long-term cardiovascular death for symptomatic patients undergoing CEA. Yet, presence of preoperative SVD does not seem valuable to predict the 30-day perioperative stroke nor long-term stroke-risk in these patients.

\section{Acknowledgements}

We thank Sara van Laar, Evelyn Velema and Joelle van Bennekom for their technical support in the Athero-Express Biobank.

\section{Financial support}

Funding was obtained from the EU 755320 Taxinomisis grant to GP, DdK and GJdB. There are no competing interests declared. 


\section{References}

1 International Carotid Stenting Study investigators, Ederle J, Dobson J, Featherstone RL, Bonati LH, van der Worp HB, et al. Carotid artery stenting compared with endarterectomy in patients with symptomatic carotid stenosis (International Carotid Stenting Study): an interim analysis of a randomised controlled trial. Lancet 2010;375(9719):985-97.

2 Writing Group, Naylor AR, Ricco J-B, de Borst GJ, Debus S, de Haro J, et al. Editor's Choice - Management of atherosclerotic carotid and vertebral artery disease: 2017 clinical practice guidelines of the European Society for Vascular Surgery (ESVS). Eur J Vasc Endovasc Surg 2018;55(1):3-81.

3 Streifler JY, Eliasziw M, Benavente OR, Alamowitch S, Fox AJ, Hachinski VC, et al. Prognostic importance of leukoaraiosis in patients with symptomatic internal carotid artery stenosis. Stroke 2002;33(6):1651-5.

4 Debette S, Beiser A, Decarli C, Au R, Himali JJ, Kelly-Hayes M, et al. Association of MRI markers of vascular brain injury with incident stroke, mild cognitive impairment, dementia, and mortality: The framingham offspring study. Stroke 2010;41(4):600-6.

5 Chutinet A, Rost NS. White matter disease as a biomarker for long-term cerebrovascular disease and dementia. Curr Treat Options Cardiovasc Med 2014;16(3):292.

6 Black S, Gao F, Bilbao J. Understanding white matter disease: Imaging-pathological correlations in vascular cognitive impairment. Stroke 2009; 40(3 Suppl): S48-52.

7 Topakian R, Barrick T, Howe F, Markus H. Blood-brain barrier permeability is increased in normal appearing white matter in patients with lacunar stroke and leukoaraiosis. J Neurol Neurosurg Psychiatry 2010;81(2):126.

8 Oksala N, Jaroma M, Pienimaki J-P, Kuorilehto T, Vanttinen T, Lehtomaki A, et al. Preoperative white matter lesions are independent predictors of long-term survival after internal carotid endarterectomy. Cerebrovasc Dis Extra 2014;4(2):122-31.

9 Ederle J, Davagnanam I, van der Worp HB, Venables GS, Lyrer PA, Featherstone RL, et al. Effect of whitematter lesions on the risk of periprocedural stroke after carotid artery stenting versus endarterectomy in the International Carotid Stenting Study (ICSS): A prespecified analysis of data from a randomised trial. Lancet Neurol 2013;12(9):866-72.

10 O'Sullivan M, Lythgoe DJ, Pereira AC, Summers PE, Jarosz JM, Williams SCR, et al. Patterns of cerebral blood flow reduction in patients with ischemic leukoaraiosis. Neurology 2002;59(3):321-6.

11 Verhoeven BAN, Velema E, Schoneveld AH, de Vries JPPM, de Bruin P, Seldenrijk CA, et al. AtheroExpress: differential atherosclerotic plaque expression of mRNA and protein in relation to cardiovascular events and patient characteristics. Rationale and design. Eur J Epidemiol 2004;19(12):1127-33.

12 Hellings WE, Peeters W, Moll FL, Piers SRD, van Setten J, Van der Spek PJ, et al. Composition of carotid atherosclerotic plaque is associated with cardiovascular outcome: a prognostic study. Circulation 2010;121(17):1941-50.

13 Barnett HJM, Taylor DW, Haynes RB, Sackett DL, Peerless SJ, et al. Beneficial effect of carotid endarterectomy in symptomatic patients with high-grade carotid stenosis. North American Symptomatic Carotid Endarterectomy Trial Collaborators. N Engl J Med 1991;325(7):445-53.

14 Barnett HJ, Taylor DW, Eliasziw M, Fox AJ, Ferguson GG, Haynes RB, et al. Benefit of carotid endarterectomy in patients with symptomatic moderate or severe stenosis. North American Symptomatic Carotid Endarterectomy Trial Collaborators. N Engl J Med 1998;339:1415-1425.

15 Halliday A, Mansfield A, Marro J, Peto C, Peto R, Potter J, et al. Prevention of disabling and fatal strokes by successful carotid endarterectomy in patients without recent neurological symptoms: randomised controlled trial. MRC asymptomatic carotid surgery trial (ACST) collaborative group. Lancet 2004;363(9420):1491502.

16 Warlow C, Farrell B, Fraser A, Sandercock P, Slattery J. Randomised trial of endarterectomy for recently symptomatic carotid stenosis: final results of the MRC European Carotid Surgery Trial (ECST). Lancet 1998;351(9113):1379-87.

17 Rothwell P, Eliasziw M, Gutnikov S, Fox A, Taylor D, Mayberg M, et al. Analysis of pooled data from the randomised controlled trials of endarterectomy for symptomatic carotid stenosis. Lancet 2003;361(9352):107-16.

18 von Allmen RS, Weiss S, Tevaearai HT, Kuemmerli C, Tinner C, Carrel TP, et al. Completeness of followup determines validity of study findings: results of a prospective repeated measures cohort study. PLoS One 2015;10(10):e0140817.

19 Kragsterman B, Pärsson H, Lindbäck J, Bergqvist D, Björck M. Outcomes of carotid endarterectomy for asymptomatic stenosis in Sweden are improving: results from a population-based registry. J Vasc Surg 2006;44(1):79-85. 
van Lammeren GW, den Ruijter HM, Vrijenhoek JEP, van der Laan SW, Velema E, de Vries J-PPM, et al. Time-dependent changes in atherosclerotic plaque composition in patients undergoing carotid surgery. Circulation 2014;129(22):2269-76.

21 Huibers A, Calvet D, Kennedy F, Czuriga-Kovács KR, Featherstone RL, Moll FL, et al. Mechanism of procedural stroke following carotid endarterectomy or carotid artery stenting within the International Carotid Stenting Study (ICSS) Randomised Trial. Eur J Vasc Endovasc Surg 2015;50(3):281-8.

22 Melkas S, Sibolt G, Oksala NKJ, Putaala J, Pohjasvaara T, Kaste M, et al. Extensive white matter changes predict stroke recurrence up to 5 years after a first-ever ischemic stroke. Cerebrovasc Dis 2012;34(3):1918.

23 Ois A, Cuadrado-Godia E, Rodriguez-Campello A, Giralt-Steinhauer E, Jimenez-Conde J, Lopez-Cuina M, et al. Relevance of stroke subtype in vascular risk prediction. Neurology 2013;81(6):575-80.

24 Oksala NKJ, Salonen T, Strandberg T, Oksala A, Pohjasvaara T, Kaste M, et al. Cerebral small vessel disease and kidney function predict long-term survival in patients with acute stroke. Stroke 2010;41(9):191420.

25 Melkas S, Putaala J, Oksala NKJ, Pohjasvaara T, Oksala A, Kaste M, et al. Small-vessel disease relates to poor poststroke survival in a 12-year follow-up. Neurology 2011;76(8):734-9.

26 Zupan M, Šabović M, Zaletel M, Popovič KŠ, Žvan B. The presence of cerebral and/or systemic endothelial dysfunction in patients with leukoaraiosis - a case control pilot study. BMC Neurol 2015;15(1):158.

27 TAXINOMISIS Project Clinical Trials.gov (2018). Stratification of patient with carotid disease (TAXINOMISIS). [online] Available at: https://clinicaltrials.gov/ct2/show/NCT03495830 [Accessed 16 Aug. 2019]

28 Wahlund LO, Barkhof F, Fazekas F, Bronge L, Augustin M, Sjögren M, et al. A new rating scale for agerelated white matter changes applicable to MRI and CT. Stroke 2001;32(6):1318-22.

29 Rots ML, van der Lugt A, de Borst GJ. Surrogate markers and reporting standards for outcome after carotid intervention. Eur J Vasc Endovasc Surg 2019;[In press]

30 Streifler JY, den Hartog AG, Pan S, Pan H, Bulbulia R, Thomas DJ, et al. Ten-year risk of stroke in patients with previous cerebral infarction and the impact of carotid surgery in the Asymptomatic Carotid Surgery Trial. Int J Stroke 2016;11(9):1020-7.

31 Pini R, Faggioli G, Longhi M, Vacirca A, Gallitto E, Freyrie A, et al. The detrimental impact of silent cerebral infarcts on asymptomatic carotid endarterectomy outcome. J Vasc Surg 2016;64(1):15-24. 


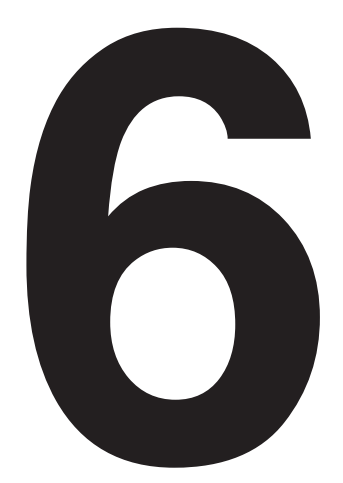

\section{Gadolium-enhancement of the aneurysm wall in extracranial carotid artery aneurysms}

CJHCM van Laarhoven*, ML. Rots*, VEC Pourier, NKN Jorritsma, T Leiner, J Hendrikse, MDI Vergouwen, GJ de Borst Am J Neuroradiol; in press 


\section{Abstract}

PURPOSE The natural history and optimal treatment of extracranial carotid artery aneurysms (ECAAs) are unknown. Gadolinium-enhancement of the aneurysm wall may reflect aneurysm wall inflammation and instability. In this study we investigated the feasibility of ECAA wall imaging and explored a potential relationship of aneurysm wall-enhancement with aneurysm growth and presence of (silent) brain infarcts and WMLs.

METHODS Fourteen conservatively treated patients with fifteen asymptomatic ECAAs underwent gadolinium-enhanced 3T-MRI at two time-points with a 12-month interval. Primary outcome was growth of the aneurysm sac $(\geq 2.0 \mathrm{~mm})$, secondary outcomes were presence of (silent) brain infarcts and white matter lesions (WMLs) at baseline and follow-up. MR-images were reviewed by two independent observers and inter- and intraobserver reproducibility was assessed.

RESULTS Seven (50\%) patients were male; median age was 54 years (range 40-66). Eleven ECAAs $(73 \%)$ were saccular (median size $11 \mathrm{~mm}$, range $5.0-38.5$ ) and four were fusiform (median size $21.5 \mathrm{~mm}$, range 10.0-40.0). Eleven out of fifteen aneurysms (73\%) exhibited gadolinium-enhancement $(\mathrm{Gd}+)$ at baseline. Four aneurysms $(27 \%)$ showed growth at follow-up imaging, two $\mathrm{Gd}+$ and two $\mathrm{Gd}$ - $(p=.245)$. Three patients $(21 \%)$ had ipsilateral brain infarcts at baseline, one of them showed new silent brain infarct at follow-up imaging (Gd+). Nine patients $(64 \%)$ showed bilateral WMLs at baseline. In three patients increased WML severity was observed at follow-up (two $\mathrm{Gd}+$ ). All observations showed excellent inter- and intraobserver reproducibility.

CONCLUSION In this explorative study we demonstrated that ECAA wall-imaging was feasible. Future well-powered studies are needed to investigate if ECAA gadoliniumenhancement predicts aneurysm growth and thrombo-embolic complications. 


\section{Introduction}

The optimal treatment and prognosis of extracranial carotid artery aneurysms (ECAAs) are still unclear. Most ECAAs are coincidental findings and a conservative approach is presently considered justified in patients with asymptomatic ECAA ${ }^{1}$. Several surgical and endovascular techniques have been developed for ECAA exclusion, but these interventions are mostly reserved for patients with neurological symptoms or with proven growth ${ }^{2,3}$.

Histopathological research revealed dissection and degeneration as the two main causes of ECAA, with the presence of inflammatory cells in the degenerative vessel wall ${ }^{4}$. MRI and histopathological studies of atherosclerotic carotid arteries have suggested that gadolinium $(\mathrm{Gd})$-enhancement reflects the density of vasa vasorum and inflammation in the arterial wall ${ }^{5,6}$. Also, MRI has been proposed as a non-invasive tool for early detection of arterial wall changes and may be useful for routine monitoring and evaluation of disease activity ${ }^{6}$. However, ECAA growth pattern and long-term risk of thrombo-embolization and subsequent stroke has yet to be elucidated.

Previous studies have shown that patients with carotid stenosis have (subclinical) microvascular cerebral damage due to micro-embolic events ${ }^{7}$. Silent brain infarction and white matter lesions (WMLs) have both been correlated with carotid plaque vulnerability and thus may be a reflection of micro-embolic events from a proximal source ${ }^{8,9}$. Silent brain infarcts and WML presence has been described to correlate with increased future risk of ischemic stroke and cognitive decline over time ${ }^{10-12}$. Assessment of both silent and symptomatic brain infarcts and WMLs may therefore be helpful surrogate markers for cerebral outcome in ECAA patients. New imaging techniques are required to assess ECAA instability in order to predict growth and cerebrovascular outcome. In this study of patients with conservatively treated asymptomatic ECAA, we aimed to investigate the feasibility of ECAA wall imaging and explored a potential relationship of aneurysm wall-enhancement with aneurysm growth and presence of (silent) brain infarcts and WMLs. 


\section{Methods}

\section{Participants}

Ethical approval for this study was provided by the Medical Research Ethics Committee of University Medical Center Utrecht, Utrecht, The Netherlands on 13 September 2016 (16-341), and all research was conducted according to the principles of the Declaration of Helsinki (59th amendment, Seoul 2008) and in accordance with the Dutch Medical Research Involving Human Subjects Act (WMO). Included patients with an ECAA were selected from the Carotid Aneurysm Registry (CAR, www.carotidaneurysmregistry.com). The registry protocol has been published elsewhere ${ }^{13}$. In short, any patient aged 18-years or older with an ECAA is included in this ongoing registry, independent of aetiology or treatment strategy. Baseline characteristics and follow-up data are collected in a prospective manner. For the present study, we selected asymptomatic patients (no symptoms for at least 1 year prior to the start of the study) from the CAR with a conservatively treated ECAA. Exclusion criteria were impaired renal function (glomerular filtration rate $<30 \mathrm{~mL} / \mathrm{min} / 1.73 \mathrm{~m}$ ), Gd contrast allergy, or pregnancy. All selected patients provided informed consent and were recruited between February and September 2017.

\section{MR imaging}

Included patients were asked to undergo MRI at moment of inclusion and one year after. Two observers (C.L., M.R.) blinded to patient characteristics and previous imaging, independently scored each of the MR scans according to a predefined protocol and case report form. Prior to scoring, both observers were trained with two test scans to familiarize with the scoring protocol. Test results were compared to overcome interpretation differences. Aneurysm location, shape and size, vessel wall-enhancement and cerebral complications were reported for two series of MR images (baseline and follow-up), with a minimum of two weeks between the measurements. For a subset of MR images, a third series was reevaluated after two weeks to determine intraobserver variability. For the final data analysis, disagreements were discussed until consensus was reached.

A 3.0 Tesla MR scanner (Ingenia, Software release 5.3, Philips Healthcare, Eindhoven, the Netherlands) was used. Images were acquired with an 18-element neurovascular coil as receiver coil. The MRI protocol consisted of a 3-D time-of-flight sequence, a contrastenhanced T1 3-D sequence (spatial resolution $0.5 \times 0.5 \times 0.5 \mathrm{~mm}$ ), and for imaging of the vessel wall a transverse T1 3D multishot spin-echo sequence of the ipsi- and contralateral extracranial carotid artery with the following parameters: field of view of 200x166x45 (by default but coverage could be adjusted in case of aneurysms $>45 \mathrm{~mm}$ ), acquired resolution $0.5 \times 0.5 \times 1.0 \mathrm{~mm}$, reconstructed resolution $0.5 \times 0.5 \times 0.5 \mathrm{~mm}, \mathrm{TR} / \mathrm{TE} 1500 / 38$. An anti-DRIVen Equilibrium technique and a minimum flip angle of $25^{\circ}$ in the variable flip angle refocusing pulse train were used for increased vessel wall contrast, fat suppression and flow suppression of blood $^{14,15}$. This sequence was done before and after contrast administration with a duration of 8:03 minutes per sequence. FLAIR and T2 brain imaging series were performed for detection of infarcts and WMLs. Gadobutrol was used as contrast agent (Gadovist, Bayer Healthcare, 
Berlin, Germany, $0.1 \mathrm{~mL} / \mathrm{kg}$ (1mmol) per kilogram body weight), flow rate $1.0 \mathrm{~mL} / \mathrm{sec}$, time between contrast administration and vessel wall imaging was 11 minutes.

\section{Aneurysm size assessment}

Aneurysm size was determined on the contrast enhanced T1-weighted images using double oblique multiplanar reconstructions, in which maximum diameters were measured. Diameters were rounded to $0.5 \mathrm{~mm}$. Fusiform or spindle-shaped ECAA was defined as $\geq 150 \%$ dilation of the arterial diameter, compared to the non-affected contralateral carotid artery diameter. In case of bilateral dilatation, the diameter of the non-affected part of the ipsilateral carotid artery was used as comparison. For saccular shaped ECAA all sizes were accepted ${ }^{1}$. In case of saccular type, length, width, and neck of the aneurysm was reported, in case of fusiform type length and width were reported (Figure 1). It was decided to define growth as a minimum difference of at least $2.0 \mathrm{~mm}$ measured in at least one of the measured dimensions (length, width, and neck), to ensure reliable assessment of growth at the acquired resolution $(0.5 \times 0.5 \times 0.5 \mathrm{~mm})$. We considered differences of $<1.0 \mathrm{~mm}$ in ECAA size clinically irrelevant.
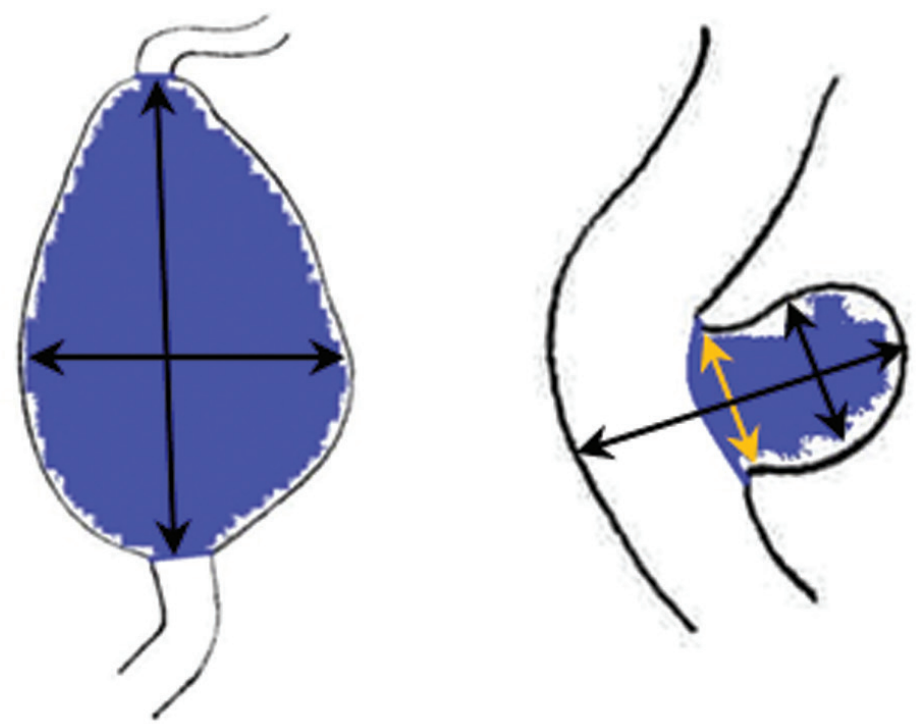

Figure 1. Schematic examples of diameter measurements of ECAA, perpendicular to the non-affected vessel. Left: fusiform ECAA length $\mathrm{x}$ width, right: saccular ECAA length $\mathrm{x}$ width $\mathrm{x}$ neck (orange).

\section{Assessment of Gd ECAA wall-enhancement}

The appearance of the aneurysms was compared before and after administration of Gd to determine the presence of wall-enhancement. Aneurysm wall-enhancement was considered present if there was a hyperintensity of the vascular wall on the MRI after Gd administration, that was not present on the MRI before Gd administration. Ipsilateral aneurysm wallenhancement was reported as well as contralateral enhancement of the internal carotid artery at the level of the aneurysm as a reference. 


\section{Assessment of brain infarcts and WML}

Presence of cortical, subcortical and lacunar infarcts on ipsi- or contralateral side was reported $^{16}$, and expressed as symptomatic or silent brain infarction. WMLs were semi quantitatively assessed at baseline and follow-up using the Fazekas scale ${ }^{17,18}$. In short, WMLs were separated according to whether hyperintense lesions were contiguous with the lateral ventricular border (periventricular lesion) or distinct and subcortical (including those in the deep white matter). Subcortical extensions from cortical and lacunar infarcts were excluded.

\section{Statistical analysis}

All observations were assessed in terms of reliability and agreement. Inter- and intraobserver reliability of each diameter was calculated by the intraclass correlation coefficient (ICC; model: two-way mixed, type: absolute agreement) with 95\% confidence interval (Cl). Bland-Altman analysis was used to assess agreement for each diameter. For nominal and binary variables (aneurysm location, shape, Gd-enhancement, and infarcts) Kappa-values were calculated, and for ordinal variables (WML) weighted Kappa was calculated ${ }^{19} .95 \% \mathrm{Cl}$ for Kappa were obtained by use of package 'psy' and 'boot' (http://cran.r-project.org) in Rstudiov3.4.1 (RStudio Team (2016). RStudio: Integrated Development for R. RStudio, Inc., Boston, MA, www.rstudio.com). Proportion of agreement was calculated for both nominal and ordinal variables ${ }^{20}$. We studied the proportion of patients with aneurysm wall-enhancement. Then, we investigated whether aneurysm wall-enhancement was associated with signs of instability during follow-up. The primary outcome was the prevalence of aneurysm wall-enhancement. Secondary outcomes were growth of the aneurysm sac during follow-up, and the presence of WMLs and (silent) brain infarcts at baseline and follow-up. Patients were compared based on Gd-enhancement by use of Fisher's Exact test and Mann Whitney $U$ test and $p<.05$ was considered statistically significant. These statistical analyses were conducted using SPSS v25.0 (IBM Corp. Released 2017. IBM SPSS Statistics for Windows, Version 25.0. Armonk, NY: IBM Corp.).

\section{Results}

Fifteen patients with 16 ECAAs were included and underwent baseline MRI. Fourteen patients (with fifteen aneurysms) underwent follow-up MRI after one year. Individual patient characteristics are listed in Table 1. The patient that was lost to follow-up was unable to participate in study activities due to clinical deterioration as a result of prior cerebral infarction. Eight out of fifteen patients (53\%) were male; median age was 54 years (range 40-66). Detailed medical history is summarized in Suppl. Table 1.

All MR observations showed excellent inter- and intraobserver reproducibility, with both ICCs and weighted Kappa's $>0.9$, and acceptable differences for agreement (Figure 2, Suppl. Table 2, Suppl. Figure 1-8). In total, 666 observations per observer were made of which $15 \%$ disagreements were discussed. 


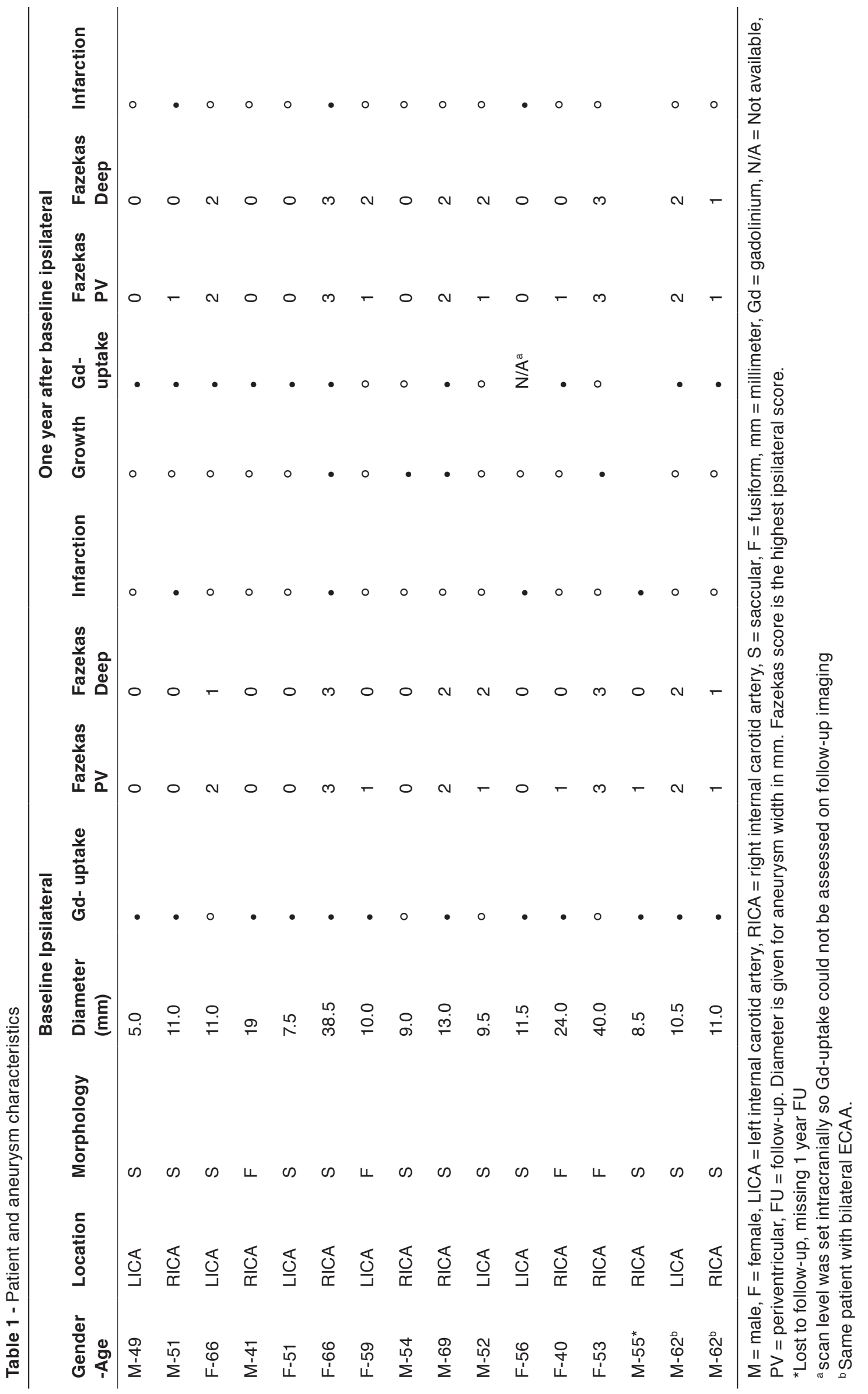


Table 2 - Aneurysm size for aneurysms with and without gadolinium enhancement.

\begin{tabular}{|c|c|c|c|}
\hline & $\begin{array}{l}\text { No gadolinium enhancement } \\
(n=4)\end{array}$ & $\begin{array}{l}\text { Gadolinium enhancement } \\
(n=12)\end{array}$ & $p$ value \\
\hline $\begin{array}{l}\text { Length baseline } \\
\text { Median (range) }\end{array}$ & $10.75(6.5-46.0)$ & $14.5(7.00-66.5)$ & .316 \\
\hline $\begin{array}{l}\text { Width baseline } \\
\text { Median (range) }\end{array}$ & $10.5(9.0-41.0)$ & $11.5(5.5-38.5)$ & .953 \\
\hline $\begin{array}{l}\text { Neck baseline a } \\
\text { Median (range) }\end{array}$ & $9.5(8.0-11.0)$ & $12.0(3.5-22.0)$ & .482 \\
\hline $\begin{array}{l}\text { Growth (length) } \\
\text { Median (range) }\end{array}$ & $1.0(-1.0-4.0)$ & $0.5(-0.5-4.5)$ & $>.999$ \\
\hline $\begin{array}{l}\text { Growth (width) } \\
\text { Median (range) }\end{array}$ & $1.0(-1.0-4.0)$ & $0.5(-0.5-4.5)$ & $>.999$ \\
\hline $\begin{array}{l}\text { Growth (neck) a } \\
\text { Median (range) }\end{array}$ & $0.0(-1.0-0.5)$ & $2.0(0.5-3.0)$ & .012 \\
\hline $\begin{array}{l}\text { Growth (any direction } \\
\geq 2 \mathrm{~mm} \text { ) } \\
N(\%)\end{array}$ & $2(50)$ & $2(16.7)$ & .245 \\
\hline
\end{tabular}

${ }^{a}$ Only saccular aneurysm patients. Bold values indicate $p<.05$.

\section{Interobserver Width}

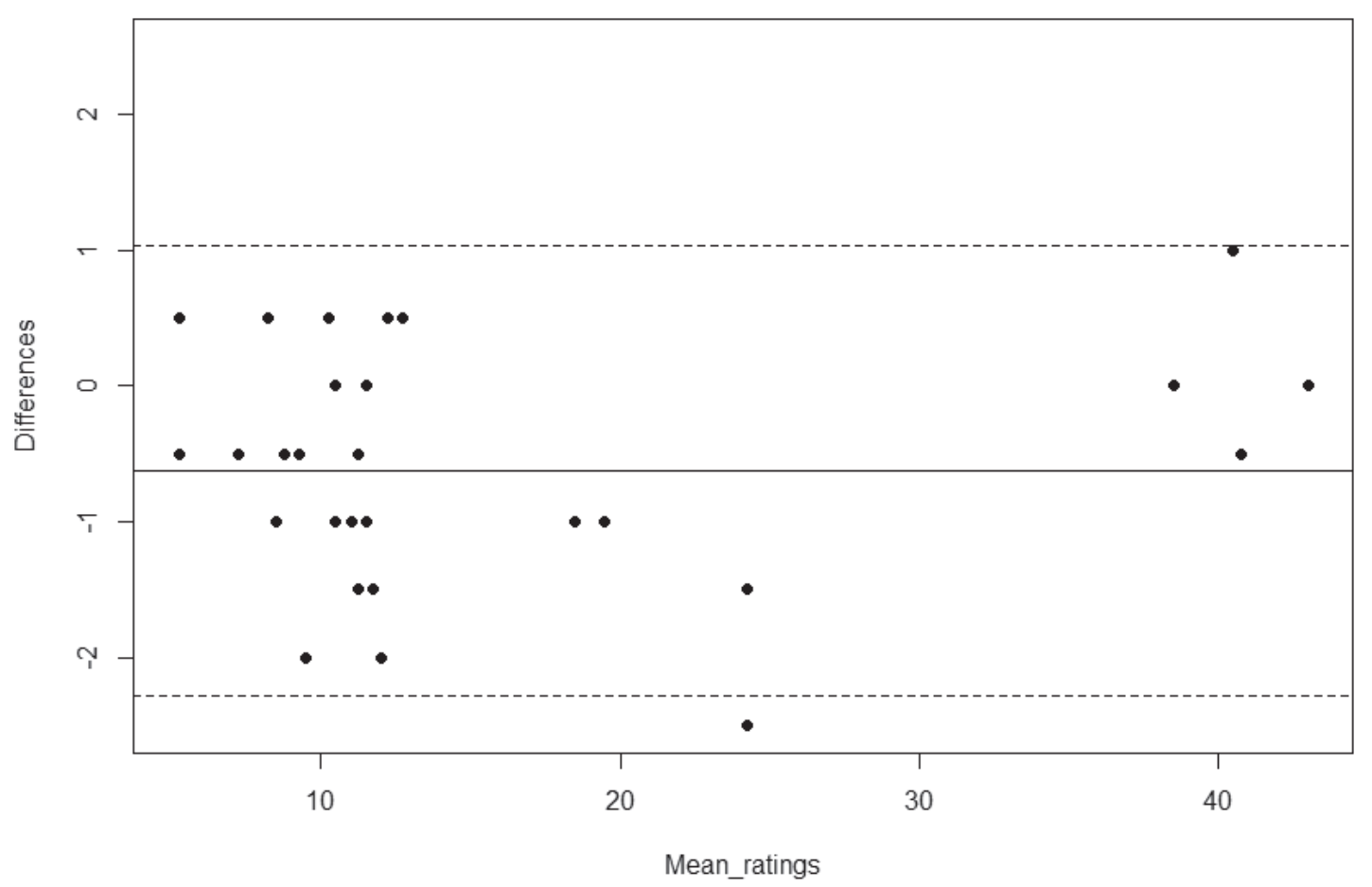

Figure 2. Bland-Altman plot of interobserver agreement. The solid line in the middle represents the mean difference of the diameter measurement between the two observers, the dashed line represents the upper and lower limits of agreement (mean difference $\pm 1.96 \times$ standard deviation). 


\section{Aneurysm analysis}

All sixteen ECAAs were located in the extracranial internal carotid artery (eight on the right, six on the left and one patient with bilateral ECAA). Twelve aneurysms were saccular $(75 \%)$ and four were fusiform. Median length was $13.5 \mathrm{~mm}$ (range 6.5-66.0mm), and median width was $11.5 \mathrm{~mm}$ (range $5.5-41.0 \mathrm{~mm})$. The median neck width was $11 \mathrm{~mm}(3.5-22.0 \mathrm{~mm})$ in saccular ECAAs. Twelve out of sixteen (75\%) aneurysms had Gd wall-enhancement at baseline. Of the fifteen aneurysms with follow-up imaging available, four (27\%) increased in size $\geq 2.0 \mathrm{~mm}$ in at least one direction at the follow-up MRI. Of these four aneurysms, one showed growth in length, width and neck, one showed only increased width, and two showed only increase in length in comparison to baseline. For further analysis, only patients with available follow-up MRA are included.

\section{Aneurysm wall Gd-enhancement}

All 28 aneurysm wall scans showed good image quality and no artifacts interfered with our assessment of wall enhancement. Out of the fifteen aneurysms with follow-up imaging, eleven exhibited ECAA wall-enhancement at baseline. Length, width and neck diameter of aneurysms with wall-enhancement compared to those without was similar (see Table 2). Of the four aneurysms that increased in size at follow-up, two (50\%) showed aneurysm wall Gd-enhancement on baseline MRI (see Table 2). When absolute differences between baseline and follow-up MRI were compared, the median increase in neck size (only saccular aneurysms) was larger in the eight $\mathrm{Gd}+$ (median 2.0 mm; range 0.5-3.0) compared to the three Gd- ECAAs (median 0.0mm; range -1.0-0.5, $p=.012$ ). Other differences over time (length and width) were comparable in patients with and without aneurysm wall-enhancement (Table 2). A total of three patients showed wall-enhancement of the contralateral carotid artery at the level of the aneurysm, all of whom also had ipsilateral enhancement of the ECAA wall. Upon follow-up, 9/15 aneurysms showed steady ipsilateral wall-enhancement and 3/15 aneurysms showed consistently no enhancement. One Gd+ aneurysm at baseline did no longer show enhancement at follow-up and one Gd- patient at baseline showed novel enhancement at follow-up whereas this was not present as baseline. For the remaining patient follow-up enhancement could not be evaluated, as the scan level was set intracranially.

Assessment of brain infarcts and WML

At baseline, two patients showed lacunar (silent) brain infarcts, one ipsilateral and one patient with multiple bilateral lacunar brain infarcts. Two other patients had cortical infarcts, one of them ipsilateral to the aneurysm and another patient with a cortical infarct contralateral to the aneurysm. In both patients cortical infarcts were clinically symptomatic, though $>1$ year prior to start of the study. No subcortical infarcts were observed and none of the infarcts were located in the area of the posterior circulation. Follow-up MRI revealed that the patient who showed multiple bilateral lacunar infarcts at baseline had a bilateral increase of the number of lacunar silent brain infarcts on follow-up imaging. Another patient had one new contralateral lacunar silent brain infarct. All patients with infarcts at baseline or follow-up showed Gdenhancement at baseline. 
The median Fazekas score at baseline was 1 (range 0-3) for ipsilateral periventricular WML and 1 for contralateral periventricular WML (range 0-3, $p=.705$ ). Median Fazekas score for deep WML was 1 (range $0-3$ ) at the site ipsilateral to the aneurysm and 0 (range $0-3$ ) on the contralateral site $(p=.816)$. Three out of fourteen patients with follow-up imaging showed an increase of Fazekas score after one year, of whom two on the ipsilateral site (one patient with increase of deep WML and one patient with increase of periventricular WML) and one patient with a bilateral increase of deep WMLs. Two out of three patients who showed an increase of WML had Gd-enhancement at baseline imaging.

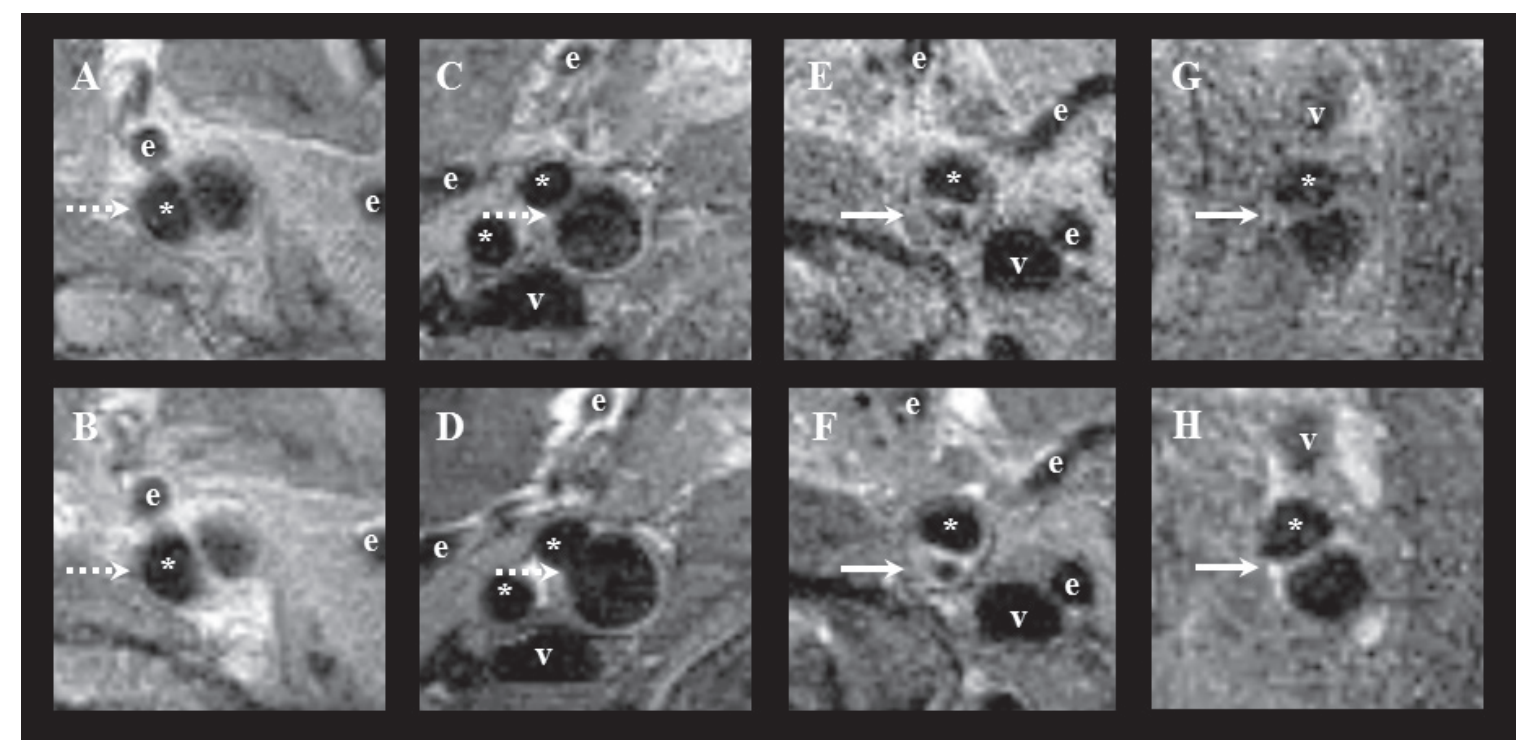

Figure 3. Examples of aneurysm wall enhancement (white arrows) on $3 T$ MRI with aneurysm wall imaging. B,D,F, and $\mathbf{H}$ show the sequence after administration of gadolinium. $\mathbf{A}$ and $\mathbf{B}$ show a 66 -yr-old woman with a $11.0 \mathrm{~mm}$ LICA aneurysm without enhancement. $\mathbf{C}$ and $\mathbf{D}$ show a 54 -yr-old man with a $9.0 \mathrm{~mm}$ RICA aneurysm without enhancement. $\mathbf{E}$ and $\mathbf{F}$ show a 51 -yr-old woman with a $7.5 \mathrm{~mm}$ LICA aneurysm with aneurysm enhancement. $\mathbf{G}$ and $\mathbf{H}$ show a 59 -yr-old woman with a $10.0 \mathrm{~mm}$ LICA aneurysm with enhancement. Abbreviations: $\mathrm{LICA}=$ left internal carotid artery, RICA = right internal carotid artery. 


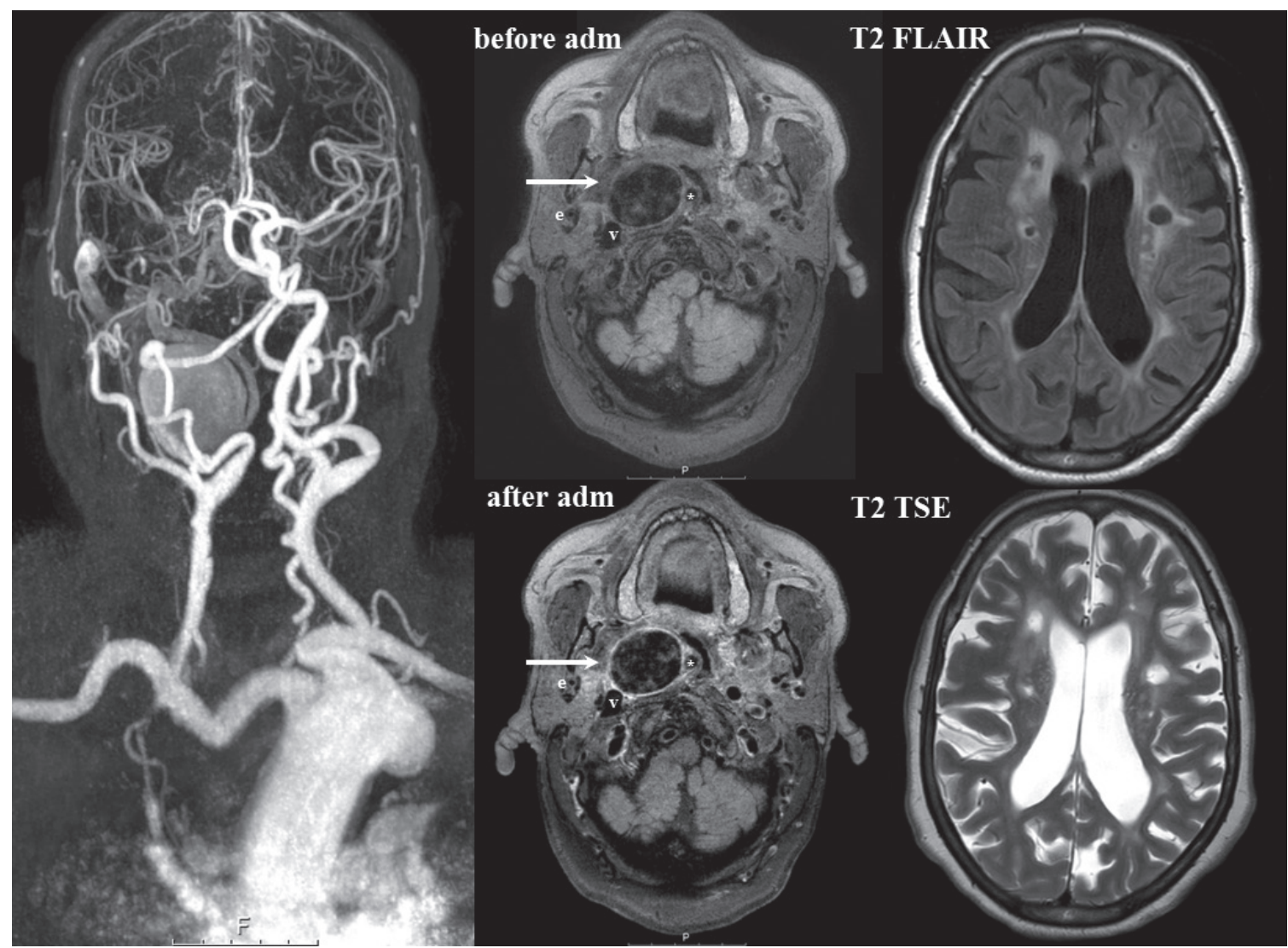

Figure 4. Overview baseline 3T Gd-enhanced MRI of 66-yr-old female with a saccular ECAA of the RICA. At baseline enhancement of the aneurysm wall was observed after contrast administration, indicated by the white arrows. T2 FLAIR \& T2 TSE showed both periventricular and deep white matter lesions Fazekas 3 and multiple bilateral lacunar infarctions. $\mathrm{Gd}$ = gadolinium; adm = administration; ECAA = extracranial carotid artery aneurysm, $\mathrm{RICA}=$ right internal carotid artery.

\section{Discussion}

In this explorative imaging study we demonstrated that ECAA wall imaging was feasible with good inter- and intraobserver reliability and agreement. The majority of aneurysms had Gd-enhancement of the ECAA wall at baseline. Aneurysm growth occurred in $27 \%$ of all aneurysms.

No earlier studies investigated wall-enhancement of extracranial carotid aneurysms. Only patients with $\mathrm{Gd}$-enhancement of atherosclerotic plaques in the carotid arteries are described ${ }^{21-23}$. It is believed that Gd-enhancement reflects both density of the vasa vasorum and endothelial permeability, and can indicate local inflammation of the vessel wall ${ }^{21-23}$. These previously performed studies used $1.5 \mathrm{~T}^{21,23}$ or $3.0 \mathrm{~T}^{22} \mathrm{MR}$ scanners, with double molality concentration of $\mathrm{Gd}$ and flow rate. The current scanning protocol was based on earlier studies investigating intracranial vessel wall imaging and reporting good image quality and little artifacts $^{14}$. Fine-tuning of the optimal imaging protocol for the carotid wall specifically, including the used sequences to suppress artefacts due to blood flow, contrast agent concentration, flow rate and time between administration and vessel wall imaging, is warranted so that uniform reporting standards can be established and study results can be compared. 
A follow-up study that included 65 intracranial aneurysms with a median follow-up of 27 months (range 20-31) showed that 4 out 19 aneurysms with wall-enhancement at baseline had instability during follow-up, compared to 0 out of 46 aneurysms without wall-enhancement ${ }^{24}$. Although our study in patients with ECAAs was not powered to detect differences in aneurysm growth between patients with and without aneurysm wall-enhancement, our follow-up results are of interest since we also found aneurysm growth in 2 out of 4 patients without wall-enhancement. Since healthy intracranial arteries lack adventitial vasa vasorum ${ }^{25,26}$, Gd-enhancement of intracranial arteries may indicate stronger local pathological neovascularization and thus inflammation than in extracranial carotid arteries ${ }^{26}$. Moreover, the discrimination between moderate or major enhancement in extracranial arteries, possibly indicating pathological neovascularization of the aneurysm wall instead of normal physiology of the vasa vasorum, is quite challenging and subject to the observer's interpretation. Although acceptable reproducibility of Gd+ was observed in the present study, a Gd+ quantitative measure would accurately increase Gd-grading. Density and thus enhancement of the vaso vasorum in normal extracranial arteries is poorly described in current literature, and between-patient and within-patient variability and its influence on wall enhancement is at this point unknown. In contrast to other vascular wall enhancement studies, the present study partly anticipated on this by also examining the contralateral artery as a reference. Of notice, only a minority (3/11) of patients with ipsilateral enhancement also had contralateral enhancement (one of whom had bilateral ECAA) so although not predictive of aneurysm growth, Gd-enhancement does seem to reflect disease activity of the vessel wall.

All three patients with ipsilateral brain infarcts showed ECAA wall-enhancement, but the majority of patients without infarcts also had aneurysm wall-enhancement. Brain infarcts and especially (small) cortical infarcts are often caused by thrombi-emboli from a proximal source such as the carotid arteries ${ }^{12}$. Silent brain infarcts have been associated with a threefold increase in risk for stroke ${ }^{12}$. In asymptomatic patients with atherosclerotic stenosis of the carotid artery those with silent brain infarcts benefit more from revascularization procedures in terms of averting stroke risk compared to those without ${ }^{11}$. ECAA patients at risk for thromboembolic complications may also be identified by assessment of ipsilateral (silent) brain infarcts, in addition to assessment of ECAA wall Gd-enhancement or aneurysm growth. Structural imaging of the brain may contribute to clinical decision making on whether or not to treat (e.g. conservatively or surgically) these patients.

We found that two-third of patients had bilateral WMLs. As there was no clear asymmetry and presence of WML did not seem restricted to patients with ECAA wall-enhancement, based on our preliminary results WMLs do not seem directly related to Gd-enhancement of the aneurysm wall. Previous studies have shown that the cause of WML is multifactorial and thrombo-embolism from a proximal source is only one of the potential causes for these radiographic findings ${ }^{8,27}$. It is striking however that these relative young patients included in our study (median age 54) show considerably often a high (bilateral) WML score compared to literature investigating WMLs in an otherwise healthy population ${ }^{28}$. WMLs have been used widely as a surrogate marker for small vessel disease and have been closely associated with risk factors for atherosclerotic disease such as hypertension ${ }^{29}$. These findings suggest that 
not so much a causal relationship exists between the presence of ECAA and WMLs, but a shared aetiology for presence of both disease expressions. WMLs have been associated with an increased risk of future stroke ${ }^{30}$ and cognitive decline ${ }^{29}$. Perhaps medical therapy in ECAA patients should be optimized to treat not only possible consequences of thrombo-embolisms, but also to treat risk factors underlying generalized vascular disease.

\section{Strengths \& Limitations}

The primary aim of our study was feasibility. Because of the small sample size, no conclusions can be drawn regarding the predictive value of wall-enhancement. Although good inter- and intra-observer reliabilities were found in our study, assessing presence of WMLs by means of the Fazekas scale can be highly dependent on expertise and experience. Also, the Fazekas scale is insensitive for subtle lesions and subtle changes over time. Other (semi-) quantitative measures, such as the rating scale for age-related white matter changes or computerized assessment of lesion volume, may be more reliable and sensitive to small changes over time. Future studies should include quantitative methods for determining white matter lesion volume so that subtle changes can be detected in an objective manner. Nevertheless, our method is easily translated to clinical practice and can straightforwardly be applied on frequently used MR sequences (FLAIR and T2). Little is known yet regarding the reliability of using MR for ECAA imaging compared to CT. Although high correlations between the two were found in studies on other type of aneurysms ${ }^{31}$, comparison of these two imaging techniques should be a topic for future research. The study sample is a reflection of clinical practice in ECAA disease, as a consequence various shapes and sizes of aneurysms with different blood flow characteristics were included. Slow blood flow within the aneurysm sac could cause 'pseudoenhancement' which potentially affected the validity and clinical applicability of our results. Future computational haemodynamic and histological studies of aneurysm wall tissue should elucidate the relation of Gd-enhancement and aneurysm wall instability further. Lastly, one might argue that conventional diameter measurements fail to indicate ECAA growth. Since appropriate imaging tools to measure geometrical differences are currently lacking, we might have missed cases that expanded in other directions than obliquely.

\section{Conclusions}

In this small explorative study, we demonstrated that extracranial carotid artery aneurysm wall imaging was feasible and Gd wall-enhancement was identified in the majority of aneurysms. Aneurysm growth was relatively common in a follow-up period of one year. Larger prospective studies are warranted to investigate the relation between ECAA wall-enhancement and aneurysm growth, (silent) brain infarcts and white matter lesions. 


\section{References}

1. Pourier VEC, Welleweerd JC, Kappelle LJ, et al. Experience of a single center in the conservative approach of 20 consecutive cases of asymptomatic extracranial carotid artery aneurysms. Eur J Neurol. 2018;25(10):1285-1289.

2. Welleweerd JC, Moll FL, De Borst GJ. Technical options for the treatment of extracranial carotid aneurysms. Expert Rev Cardiovasc Ther. 2012;10(7):925-931.

3. Welleweerd JC, Den Ruijter HM, Nelissen BGL, et al. Management of Extracranial Carotid Artery Aneurysm. Eur J Vasc Endovasc Surg. 2015;50(2):141-147.

4. Welleweerd JC, Nelissen BGL, Koole D, et al. Histological analysis of extracranial carotid artery aneurysms. PLoS One. 2015;10(1):1-9.

5. Backes D, Hendrikse J, van der Schaaf I, et al. Determinants of Gadolinium-Enhancement of the Aneurysm Wall in Unruptured Intracranial Aneurysms. Neurosurgery. 2017;83(4):719-725.

6. Catherine Oppenheim, Naggara O, Touzé E, et al. High-Resolution MR Imaging of the Cer- vical Arterial Wall: What the Radiologist Needs to Know. RadioGraphics. 2009;29:1413-1431.

7. Jayasooriya G, Thapar A, Shalhoub J, et al. Silent cerebral events in asymptomatic carotid stenosis. J Vasc Surg. 2011;54(1):227-236.

8. Bouwhuijsen QJA Van Den, Vernooij MW, Verhaaren BFJ, et al. Carotid plaque morphology and ischemic vascular brain disease on MRI. Am J Neuroradiol. 2017;38(9):1776-1782.

9. Altaf N, Ed M, Morgan PS, et al. Hyperintensities Are Associated with Carotid Intraplaque Hemorrhage. Radiology. 2008;248(1).

10. Gensicke H, Van Der Worp HB, Nederkoorn PJ, et al. Ischemic brain lesions after carotid artery stenting increase future cerebrovascular risk. J Am Coll Cardiol. 2015;65(6):521-529.

11. Streifler JY, den Hartog AG, Pan S, et al. Ten-year risk of stroke in patients with previous cerebral infarction and the impact of carotid surgery in the Asymptomatic Carotid Surgery Trial. Int J Stroke. 2016;11(9):10201027.

12. Vermeer SE, Prins ND, den Heijer T, et al. Silent Brain Infarcts and the Risk of Dementia and Cognitive Decline. N Engl J Med. 2003;348(13):1215-1222.

13. Welleweerd JC, Bots ML, Kappelle LJ, et al. Rationale and design of the extracranial Carotid artery Aneurysm Registry (CAR). J Cardiovasc Surg (Torino). 2018;59(5):692-698.

14. Dieleman N, Yang W, Van Der Kolk AG, et al. Qualitative evaluation of a high-resolution 3D multi-sequence intracranial vessel wall protocol at 3 tesla MRI. PLoS One. 2016;11(8):1-11.

15. Yang $\mathrm{H}$, Zhang X, Qin Q, et al. Improved cerebrospinal fluid suppression for intracranial vessel wall MRI. J Magn Reson Imaging. 2016;44(3):665-672.

16. de Leeuw F, de Groot J, Bots M, et al. Carotid atherosclerosis and cerebral white matter lesions in a population based magnetic resonance imaging study. J Neurol. 2000;247(4):291-296.

17. Fazekas F, Chawluk JB, Alavi A, et al. Mr Signal Abnormalities At 1.5-T in Alzheimer Dementia and Normal Aging. Am J Roentgenol. 1987;149(2):351-356.

18. Kim KW, MacFall JR, Payne ME. Classification of white matter lesions on magnetic resonance imaging in the elderly. Biol Psychiatry. 2008;64(4):273-280.

19. Kottner J, Audige L, Brorson S, et al. Guidelines for Reporting Reliability and Agreement Studies (GRRAS) were proposed. Int J Nurs Stud. 2011;48(6):661-671.

20. de Vet HCW, Terwee CB, Knol DL, et al. When to use agreement versus reliability measures. $J$ Clin Epidemiol. 2006;59(10):1033-1039.

21. Aoki S, Aoki K, Ohsawa S, et al. Dynamic MR imaging of the carotid wall. J Magn Reson Imaging. 1999;9(3):420-427.

22. Qiao Y, Etesami M, Astor BC, et al. Carotid plaque neovascularization and hemorrhage detected by MR imaging are associated with recent cerebrovascular ischemic events. Am J Neuroradiol. 2012;33(4):755760.

23. Kerwin WS, Brien KDO, Ferguson MS, et al. Inflammation in Carotid Atherosclerotic Plaque: A Dynamic Contrast-enhanced MR Imaging Study. Radiology. 2006;241(2):459-468.

24. Vergouwen MDI, Backes D, van der Schaaf IC, et al. Gadolinium Enhancement of the Aneurysm Wall in Unruptured Intracranial Aneurysms Is Associated with an Increased Risk of Aneurysm Instability: A Follow-Up Study. Am J Neuroradiol. 2019:1-5.

25. Krings T, Mandell DM, Kiehl TR, et al. Intracranial aneurysms: From vessel wall pathology to therapeutic approach. Nat Rev Neurol. 2011;7(10):547-559.

26. Portanova A, Hakakian N, Mikulis DJ, et al. Vasa vasorum 2013 insights and implications for imaging. Radiology. 2013;267(3):667-679.

27. Potter GM, Doubal FN, Jackson CA, et al. Lack of association of white matter lesions with ipsilateral carotid artery stenosis. Cerebrovasc Dis. 2012;33(4):378-384. 
28. Leeuw F-E De, Groot JC De, Achten E, et al. Prevalence of cerebral white matter lesions in elderly people: A population based magnetic resonance imaging study. The Rotterdam Scan Study. J Neurol Neurosurg Psychiatry. 2001;70(1):9-14.

29. Van Dijk EJ, Prins ND, Vrooman HA, et al. Progression of cerebral small vessel disease in relation to risk factors and cognitive consequences: Rotterdam scan study. Stroke. 2008;39(10):2712-2719.

30. Streifler JY, Eliasziw M, Benavente OR, et al. Prognostic importance of leukoaraiosis in patients with symptomatic internal carotid artery stenosis. Stroke. 2002;33(6):1651-1655.

31. Acar T, Karakas AB, Ozer MA, et al. Building Three-Dimensional Intracranial Aneurysm Models from 3D-TOF MRA: a Validation Study. J Digit Imaging. 2019:963-970. 


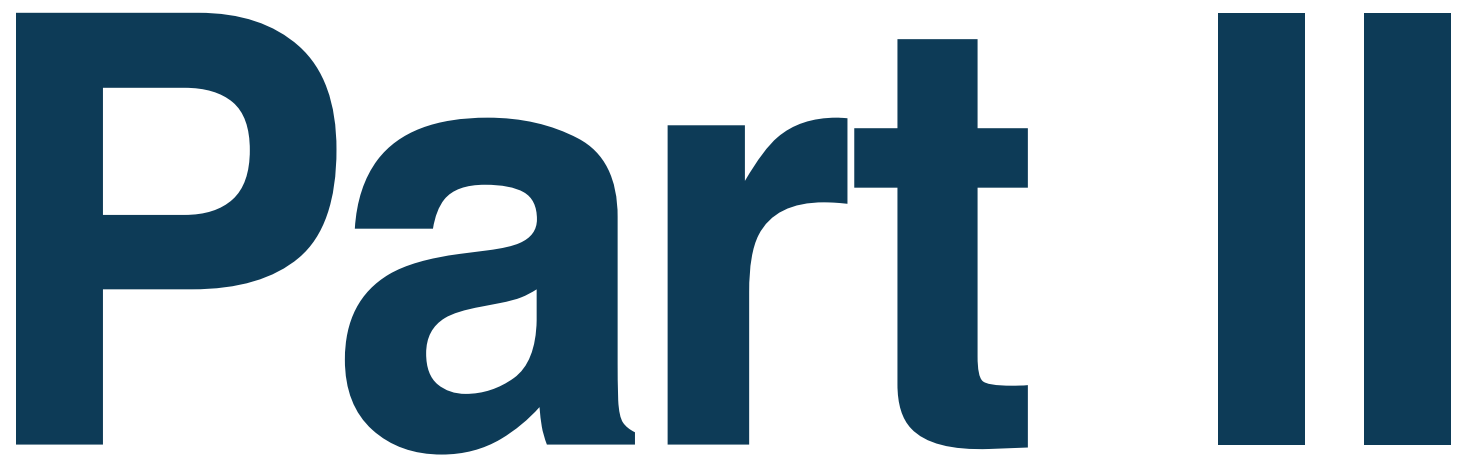

Changes in cerebral haemodynamics in patients undergoing carotid intervention 


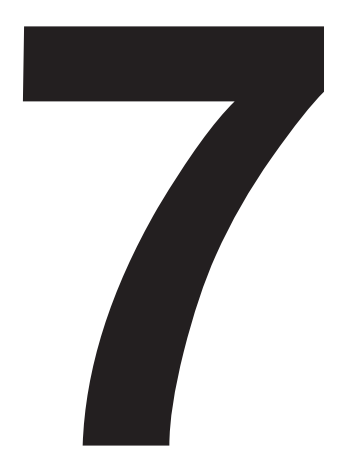

\section{Intraoperative hypotension is a risk factor for postoperative silent brain ischaemia in patients with preoperative hypertension undergoing carotid endarterectomy}

ML Rots*, LMM Fassaert*, LJ Kappelle, MCH de Groot, S Haitjema, LH Bonati, WA van Klei, GJ de Borst Eur J Vasc Endovasc Surg 2019; in press 


\section{Abstract}

OBJECTIVE Intraoperative haemodynamic instability during carotid endarterectomy (CEA) has been associated with an increased risk of procedural stroke. Diffusion-weighted imaging (DWI) lesions have been proposed as a surrogate marker for perioperative silent cerebral ischaemia. This study aimed to investigate the relation between perioperative blood pressure (BP) and presence of postoperative DWI-lesions in patients undergoing CEA.

METHODS A retrospective analysis was performed based on symptomatic CEA-patients included in the MRI-substudy of the International Carotid Stenting Study. Relative intraoperative hypotension was defined as a decrease of intraoperative systolic BP $\geq 20 \%$ compared to preoperative ('baseline') BP, absolute hypotension was defined as a drop in systolic BP $<80 \mathrm{mmHg}$. Primary endpoint was presence of any new DWI lesions on postoperative MRI (DWI-positive). Occurrence and duration of intraoperative hypotension was compared between DWI-positive and DWI-negative patients as was the magnitude of the difference between pre- and intraoperative BP.

RESULTS 55 symptomatic CEA-patients were included of whom eight were DWI-positive. DWI-positive patients had a significantly higher baseline systolic $(186 \pm 31$ vs $158 \pm 27 \mathrm{mmHg}$, $p=.011)$ and diastolic BP $(95 \pm 15$ vs $84 \pm 13 \mathrm{mmHg}, p=.046)$ as compared to DWI-negative patients. Other preoperative characteristics did not differ. Relative intraoperative hypotension compared with baseline occurred in 53/55 patients (median duration 34 minutes; range 0 - 174). Duration of hypotension did not significantly differ between the groups $(p=.088)$. Mean systolic intraoperative BP as compared to baseline, revealed a larger drop of BP $(-37 \pm 29 \mathrm{mmHg})$ in DWI-positive compared to DWI-negative patients $(-14 \pm 26 \mathrm{mmHg}, p=.024)$. Absolute intraoperative systolic BP values did not differ between the groups.

CONCLUSION In this exploratory study, high preoperative BP and a larger drop of intraoperative BP were associated with periprocedural cerebral ischaemia as documented with DWI. These results call for confirmation in an adequately sized prospective study, as they suggest important consequences for perioperative haemodynamic management in carotid revascularization. 


\section{Introduction}

Haemodynamic disturbances such as bradycardia and extreme hypotension are an important pathophysiological mechanism of stroke after carotid endarterectomy (CEA) and carotid artery stenting (CAS) ${ }^{1,2}$. However, little is known about the risks of more subtle variations in perioperative blood pressure (BP) on postoperative stroke. In addition, in patients undergoing CEA, cerebral autoregulation and collateral circulation may be compromised, which may lead to cerebral hypoperfusion even in the absence of systemic haemodynamic disturbances ${ }^{3-6}$. Cerebral hypoperfusion may lead directly to hypoxia and subsequently ischaemia in areas of the brain or may interact with embolic mechanisms by inhibition of the washout of artery-to-artery emboli during $\mathrm{CEA}^{7,8}$. Micro-emboli during the dissection procedure of the carotid artery are correlated with postoperative development of new ischaemic lesions on diffusion-weighted imaging (DWI) after CEA, which may be aggravated by hypoperfusion ${ }^{9}$.

The occurrence of silent periprocedural ischaemic brain lesions on MR-DWI after revascularization of the carotid artery, either with CAS or CEA, has been a topic of increasing interest ${ }^{10,11}$. A considerably high incidence of new postprocedural DWI lesions is reported after both CAS (50\%) and CEA (17\%) $)^{11-14}$. Ischaemic areas can be identified on DWI within minutes of a hypoxic episode and thus DWI lesions are a useful surrogate marker for early cerebral complications. The presence of new DWI lesions after CEA and CAS is associated with a higher risk for periprocedural stroke $\mathrm{e}^{15}$ and with a higher risk for future stroke or TIA after $\mathrm{CAS}^{10}$ or non-cardiac surgery in general ${ }^{16}$. Additionally, postoperative brain lesions have been associated with cognitive decline and dementia ${ }^{16-18}$. A small number of studies investigating the relation between intraoperative BP and its effect on the development of new DWI lesions have been performed in CEA patients ${ }^{9,19,20}$. Most of these studies report merely on severe intraoperative haemodynamic depression (e.g. severe hypotension, bradycardia, asystole) instead of investigating more subtle variations in BP. We hypothesized that patients with intraoperative hypotension have an increased risk for the development of new silent ischaemic lesions. Therefore, we aimed to explore the association between intraoperative BP-measurements and the occurrence of new silent ischaemic lesions on MR-DWI characteristics in patients undergoing CEA.

\section{Methods}

A retrospective analysis was performed based on prospectively collected data of patients included simultaneously in the MRI-substudy of the International carotid stenting study (ICSS $^{14}$ and the Utrecht Patient Oriented Database (UPOD) ${ }^{11}$. The structure and content of UPOD have been described in more detail previously ${ }^{21}$. Patients randomized in the ICSS for CEA in the University Medical Center (UMC) Utrecht were included. Patients included in the ICSS (between October 2003 and October 2008) had a symptomatic carotid stenosis $>50 \%$ deemed to require treatment. Exclusion criteria were: previous revascularization in 
the randomized artery, contraindications for either treatment and planned major surgery ${ }^{13}$. UPOD is a data registry curated by medical experts comprising data on patient characteristics, hospital discharge diagnoses, medical procedures, medication orders and laboratory tests for all patients treated in the UMC Utrecht. UPOD data acquisition and management is in accordance with current regulations concerning privacy and ethics. BP-measurements were related to MR-DWI outcome parameters (primary outcome measure) as well as postoperative adverse events (stroke, myocardial infarction, cardiovascular death - secondary outcome measures).

\section{Blood pressure measurement}

Intraoperative blood pressure $\left(\mathrm{BP}_{\text {intraop }}\right)$ registration, both invasive radial artery BPmeasurements as well as oscillometric non-invasive BP-measurements (NIBP) of brachial artery, were compared to NIBP measured prior to surgery ('baseline'). $\mathrm{BP}_{\text {intraop }}$ was measured in the operating room (OR), non-invasively as well as invasively (112Hz, 60s-samples). Measurements were obtained by Datex-Ohmeda S/5 anaesthesia monitor (GE, Healthcare, Waukesha, WI) and Arbocath 20G (Hospira, Lake Forest, IL, USA), respectively and stored in the Anaesthesia Information Management System (Anstat, Carepoint Nederland B.V.,Ede, The Netherlands).

Two types of baseline $\mathrm{BP}$-measurements were used: preoperative- $\mathrm{BP}\left(\mathrm{BP}_{\text {preop }}\right)$ and preinduction- $\mathrm{BP}\left(\mathrm{BP}_{\text {preind }}\right)$. $\mathrm{BP}_{\text {preop }}$ was defined as $\mathrm{BP}$ measured on the outpatient clinic or ward no longer than one month before surgery, using a mean of all available $\mathrm{BP}_{\text {preop }}$ measurements. $\mathrm{BP}_{\text {preind }}$ was defined as an awake oscillometric non-invasive BP-measurement in the OR before administration of any medication used during induction of anaesthesia. Intraoperative hypotension was defined both as a decrease of $\geq 20 \%$ below baseline (relative hypotension) or an absolute drop in systolic BP below $80 \mathrm{mmHg}$ (absolute hypotension) ${ }^{22}$. Intraoperative hypertension was defined as an increase of $\geq 20 \%$ of systolic BP compared to baseline values ${ }^{23} . \mathrm{BP}_{\text {intraop }}$ was analysed from the time of anaesthesia induction until emergence. For further data analysis, results of $\mathrm{BP}_{\text {intraop }}$ will be compared to $\mathrm{BP}_{\text {preop }}$ and $\mathrm{BP}_{\text {preind }}$ in a separate fashion. The occurrence of intraoperative hypotension, intraoperative hypertension and the duration of these changes in BP were associated with the presence of DWI lesions as was the magnitude of the differences in $\mathrm{BP}$. Variation in $\mathrm{BP}_{\text {intrap }}$ was described by calculation of the standard deviation (SD) of all (invasive radial artery) systolic and diastolic $\mathrm{BP}_{\text {intraop }}$ measurements within a patient.

\section{CEA protocol}

All patients underwent CEA under general anaesthesia and, in $53 \%$ of patients, an additional cervical plexus block. CEA was performed by an experienced vascular surgeon or a vascular trainee under the supervision of a vascular surgeon. All patients were neurologically monitored during surgery by electroencephalogram (EEG) and transcranial Doppler (TCD). An intraluminal shunt was placed selectively in case of EEG asymmetry or a decrease of $>70 \%$ of mean flow velocity in the middle cerebral artery (MCA) measured by TCD. Intraoperative target BP was between 100 and $120 \%$ of $\mathrm{BP}_{\text {preind }}$ during clamping. Postoperatively, all patients 
were admitted to the recovery unit for haemodynamic and neurological monitoring for at least 6 hours.

\section{MRI}

MRI was performed on day 1-7 prior to surgery (preoperative MRI) and day 1-3 after surgery (postoperative MRI). DWI sequences were used at each scan to detect acute ischaemic brain lesions. MR field strength was 1.5 or 3 Tesla. A neurologist and a neuroradiologist, both masked to intraoperative features analysed all scans as previously described ${ }^{14}$. The number and volume of hyperintense lesions on DWI were measured. Volumes of separate lesions were calculated by measuring lesion diameters in three axes, converted to $\mathrm{ml}$. Lesions were considered separate if there was no continuity on the same slice as well as on adjacent slices. Primary outcome parameter was presence of any new postoperative (ipsi- or contralateral) ischaemic lesion on MR-DWI, secondary outcome parameter was DWI lesion volume and count.

\section{TCD}

Intraoperative routine TCD monitoring was performed for cerebrovascular monitoring whenever acoustic temporal bone window was adequate (preoperatively assessed). A pulsed Doppler transducer was placed over the temporal bone to insonate the main stem of the ipsilateral MCA, with the TCD transducer being fixed with a head frame and flow velocity measured continuously. The increase or decrease of flow velocity after cross-clamping of the carotid artery was calculated as a percentage compared to values prior to cross-clamping. Microemboli during the procedure were scored and registered as present or absent by an experienced clinical neurophysiologist.

\section{Statistics}

Data were inspected for patterns of missing values. The proportion of randomly missing values for baseline characteristics was less than $4 \%$. Differences in binary characteristics were analysed with Pearson's Chi-square between DWI-positive and DWI-negative patients. Differences in continuous parameters were calculated with a student's t-test or a MannWhitney $\mathrm{U}$ test, as appropriate. Correlations between continuous parameters and continuous outcome (DWI lesion volume and count) were calculated using the Pearson correlation. Continuous data are provided as mean ( \pm standard deviation) or median (range), as appropriate and categorical variables as $n$ (percentage). A $p$-value of <.05 was considered statistically significant. Differences between $\mathrm{BP}_{\text {preop }}$ and $\mathrm{BP}_{\text {intraop }}$ (non-invasive and invasive, systolic/ diastolic and mean arterial pressure (MAP)) were evaluated. SPSS 25.0 (SPSS Inc, Chicago, Illinois, USA) was used for all statistical analysis. 


\section{Results}

Fifty-seven patients met the inclusion criteria, of which 55 had intraoperative BP-measurements available. Eight patients had DWI lesions on the postoperative MR-DWI that were not present on preoperative MR imaging (15\%, DWI-positive group) in comparison to 47 patients without any new DWI lesions (85\%, DWI-negative group). Five out of eight only had new ipsilateral lesions, three had both ipsi- and contralateral lesions (for example, see Figure 1). Mean lesion volume was $3.9 \mathrm{~mL}$ (median 0.37 , range from 0.05 to 19.3). The median number of new lesions in DWI-positive patients was 3.5 (range 1-14). DWI-positive patients had a significantly higher $\mathrm{BP}_{\text {preop }}$ systolic (186 \pm 31 vs $\left.158 \pm 27 \mathrm{mmHg}, p=.011\right)$ and diastolic BP $(95 \pm 15$ vs $84 \pm 13 \mathrm{mmHg}$, $p=.046$ ) when compared to DWI-negative patients. Other baseline characteristics did not show statistically significant differences between the groups (Table 1). All but one DWI-positive patients had a systolic $\mathrm{BP}_{\text {preop }}$ of $\geq 170 \mathrm{mmHg}$. Preoperative imaging showed DWI-lesions in 24 out of 55 patients (43.6\%), no differences in preoperative systolic or diastolic BP was observed between patients with and without preoperative lesions and preoperative DWI-lesion count did not correlate with post-operative DWI lesion count and volume (see supplemental data).
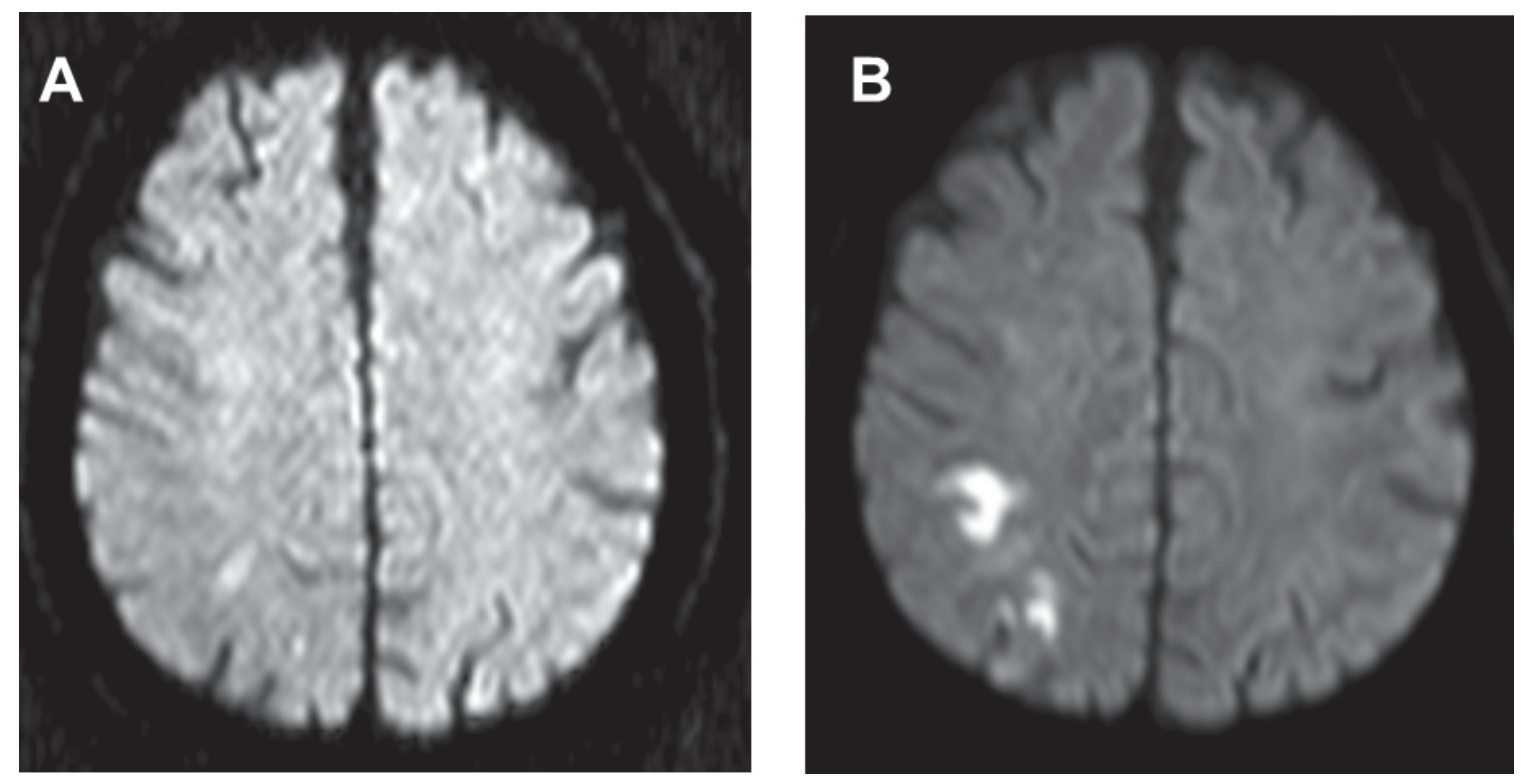

Figure 1. Preoperative DWI scan (A) compared to postoperative DWI scan (B) of patient undergoing carotid endarterectomy (CEA): In this patient, a total of 14 (13 ipsilateral and 1 contralateral) lesions were seen on MR-DWI that were not present on the preoperative scan with a total volume of $19.2 \mathrm{~mL}$.

\section{Intraoperative BP-measurements}

A mean number of 138 minutes of intraoperative invasive radial artery BP-measurements were available per surgical procedure (median 137, range 78-213). Absolute values of $\mathrm{BP}_{\text {intraop }}$ and relative values related to $\mathrm{BP}_{\text {preind }}$ and $\mathrm{BP}_{\text {preop }}$ were compared between DWI-negative and DWI-positive patients (Tables 2 and 3). An example of perioperative BP of a DWI-negative and DWI-positive patient is presented in Figure 2. 
Table 1 Baseline characteristics of DWI-negative compared to DWI-positive patients.

\begin{tabular}{|c|c|c|c|}
\hline Characteristic & $\begin{array}{l}\text { DWI-negative } \\
\mathrm{N}=47\end{array}$ & $\begin{array}{l}\text { DWI-positive } \\
\mathrm{N}=8\end{array}$ & p-value \\
\hline Age & $68.7 \pm 1.4$ & $67.24 \pm 2.5$ & .674 \\
\hline Male gender & $33(70.2)$ & $6(75)$ & .783 \\
\hline Hypertension & $34(72.3)$ & $5(62.5)$ & .571 \\
\hline Systolic blood pressure & $158 \pm 28$ & $186 \pm 31$ & .010 \\
\hline Diastolic blood pressure & $84 \pm 13$ & $95 \pm 15)$ & .045 \\
\hline Anti-hypertensives use & $35(74.5)$ & $6(75)$ & .975 \\
\hline Diabetes Mellitus & $11(23.4)$ & $1(12.5)$ & .490 \\
\hline Hypercholesterolemia & $29(61.7)$ & $6(75.0)$ & .470 \\
\hline Statin/other lipid lowering drugs used & $41(87.2)$ & 7 (87.5) & .983 \\
\hline Antiplatelet use & $43(91.5)$ & 7 (87.5) & .717 \\
\hline Oral anticoagulants & $5(10.6)$ & $1(12.5)$ & .876 \\
\hline Currently smoking & $17(36.2)$ & $2(25.0)$ & .539 \\
\hline BMI & $25.0(23.9-27.1)$ & $26.6(23.3-28.2)$ & .495 \\
\hline History of CAD & $10(21.3)$ & $4(50.0)$ & .085 \\
\hline History of PAOD & $8(17.0)$ & $1(12.5)$ & .749 \\
\hline Qualifying symptom $=$ hemispheric stroke & $15(31.9)$ & $2(25.0)$ & .696 \\
\hline Major stroke & $9(17.0)$ & $0(0)$ & .313 \\
\hline Minor stroke & $7(14.9)$ & $2(25)$ & \\
\hline Cerebral TIA & $25(53.2)$ & $6(75)$ & \\
\hline Amaurosis fugax & $7(14.9)$ & $0(0)$ & \\
\hline Stenosis grade $\geq 70 \%$ & $43(91.5)$ & $8(100)$ & .734 \\
\hline Artery operated on (right side) & $29(61.7)$ & $6(75.0)$ & .522 \\
\hline Contralateral stenosis $\geq 50 \%$ & $15(31.9)$ & $0(0)$ & .061 \\
\hline Contralateral occlusion & $3(6.4)$ & $0(0)$ & .462 \\
\hline Additional cervical plexus block & $26(55)$ & $3(38)$ & .351 \\
\hline Use of intraoperative shunting & $3(6.4)$ & $2(25.0)$ & .090 \\
\hline Carotid clamping time in minutes & $37(13-104)$ & $41(21-51)$ & .782 \\
\hline
\end{tabular}

Data are given $\mathrm{n}(\%)$, as mean \pm standard deviation (SD) in case of normally distributed data, or as median (range) in case of not normally distributed data. Hypertension was defined as previously diagnosed by an MD or use of antihypertensive drugs. Systolic and diastolic blood pressure were measured at intake/hospital admission, in case not available the first available pre-operative blood pressure was used. Diabetes mellitus was defined as previously diagnosed by an MD or use of antidiabetic medication. LDL; mmol/L measured within 1 month prior to surgery. Antiplatelet use; dipyridamole, acetylsalicylic, carbasalate calcium or clopidogrel, anti-coagulation; coumarone or direct acting oral anticoagulant. History of CAD was defined as a composite of angina pectoris, myocardial infarction, percutaneous coronary interventions or coronary bypass surgery. PAOD; peripheral artery disease was defined as a history of peripheral interventions or intermittent claudication or ankle-brachial index<0.7. Use of intraoperative shunting was based on neuromonitoring. Carotid clamping times were compared for a selection of patients in whom no shunting was performed. 
A

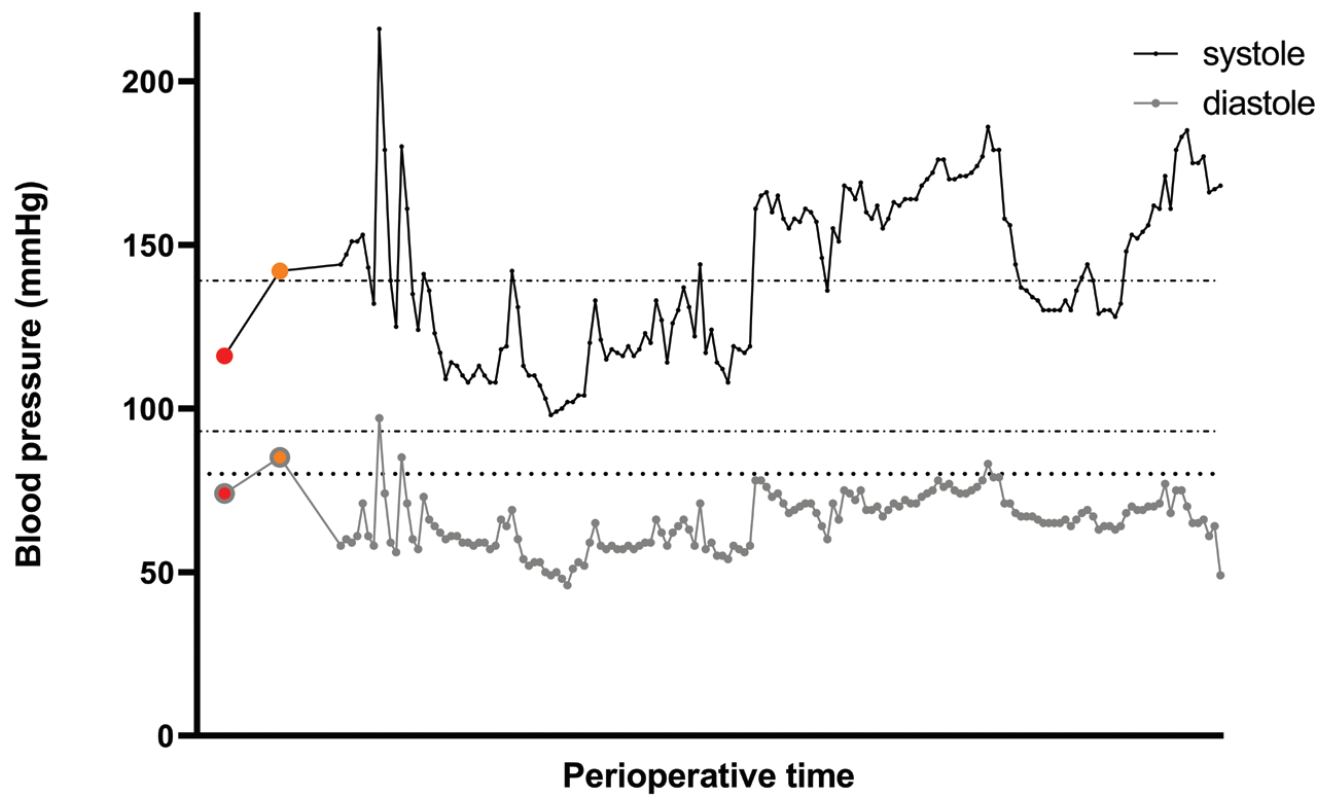

B

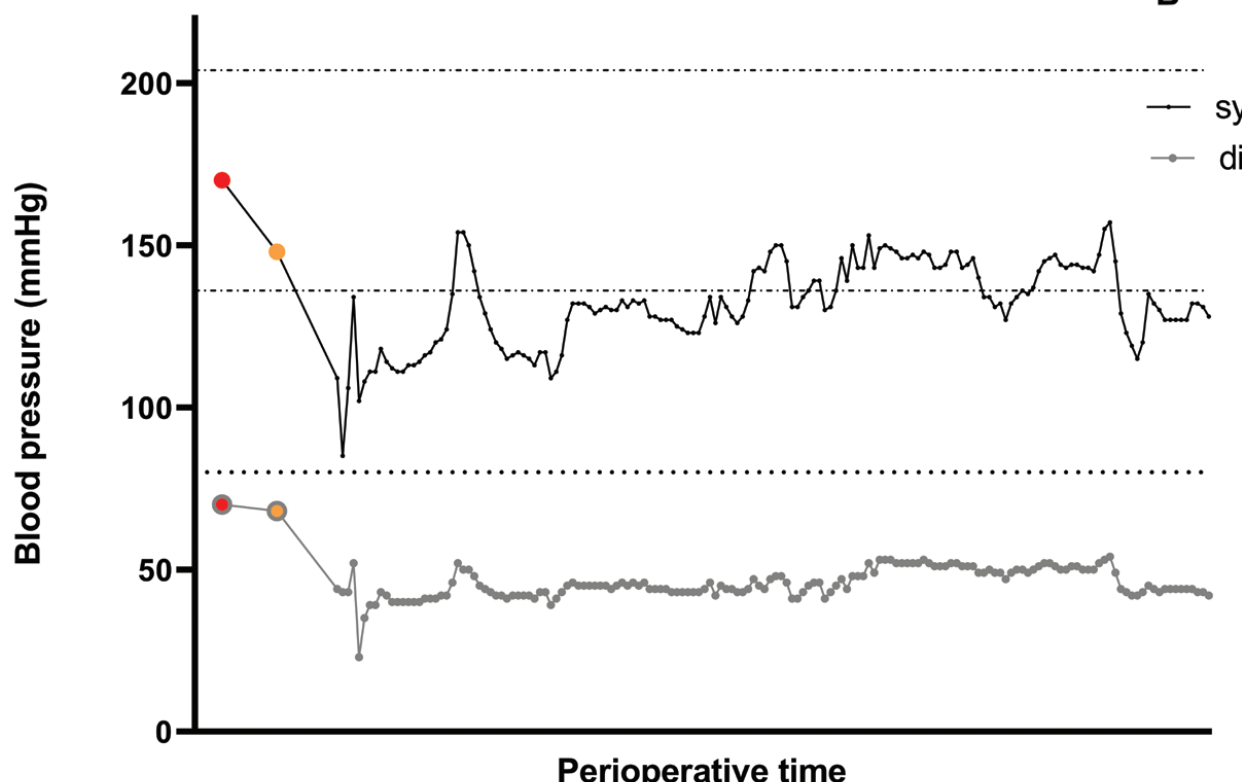

Perioperative time

Figure 2. Typical example of blood pressure course in perioperative period in two patients undergoing carotid endarterectomy (CEA), with panel A representing a DWI-negative patient and panel $\mathrm{P}$ a DWI-positive patient: In each of the panels, the red dot represents $\mathrm{BP}_{\text {preop }}$, the orange dot represents $\mathrm{BP}_{\text {preind }}$ and subsequent measurements reflect $\mathrm{BP}_{\text {intraop }}$. The two (upper) dashed horizontal lines represent the relative hypo- $(\geq 20 \%$ decrease) and hypertension ( $\geq 20 \%$ increase) limits compared to $\mathrm{BP}_{\text {preop }}$. The lower dotted line represents the cut-off for absolute hypotension $(80 \mathrm{mmHg})$. The graph in panel B illustrates how the preinduction blood pressure, which is considerably decreased compared to the earlier preoperative blood pressure, determines intraoperative target BP and very often exceeds the limit of relative hypotension whereas both patients never cross the limit of absolute hypotension. The two example patients were randomly selected from each group. 


\section{Absolute $\mathrm{BP}_{\text {intraop }}$ values}

There was no difference in systolic or diastolic mean absolute $\mathrm{BP}_{\text {intraop }}$ between DWI-negative and DWI-positive patients (Table 2). Absolute hypotension occurred in 13 patients, all of whom were DWI-negative and lasted between 1 and 7 minutes. No significant differences were found when duration of absolute hypotension was compared between DWI-negative and DWI-positive patients. Intraoperative variability of systolic and diastolic BP did not differ between the groups either.

Table 2 Results of absolute intraoperative blood pressure measurements of DWI-negative compared to DWI-positive patients

\begin{tabular}{llll}
\hline BP parameter & $\begin{array}{l}\text { DWI-negative } \\
(\mathbf{n}=\mathbf{4 7})\end{array}$ & $\begin{array}{l}\text { DWI-positive } \\
(\mathbf{n}=\mathbf{8})\end{array}$ & $\boldsymbol{p}$-value \\
\hline Absolute $\mathrm{BP}$ & & & .399 \\
Mean systolic $\mathrm{BP}_{\text {intraop }}, \mathrm{mmHg}$ & $145 \pm 15$ & $149 \pm 20$ & .655 \\
Mean diastolic $\mathrm{BP}_{\text {intraop }}, \mathrm{mmHg}$ & $62 \pm 8$ & $66 \pm 10$ & .224 \\
$\begin{array}{l}\text { Duration of absolute hypotension } \\
\text { Systolic } \mathrm{BP}_{\text {intraop }}<80 \mathrm{mmHg} \text { in minutes }\end{array}$ & $0(0-7)$ & $0(0-0)$ & .459 \\
Variability & & & .788 \\
Variability of systolic $\mathrm{BP}_{\text {intraop }}, \mathrm{SD}$ & $24.5(16.2-35.2)$ & $25.7(13.6-36.3)$ & $11.3(4.4-15.5)$ \\
Variability of diastolic $\mathrm{BP}_{\text {intraop}}, \mathrm{SD}$ & $10.3(5.4-31.1)$ & & \\
\hline
\end{tabular}

Data are given as mean \pm standard deviation (SD) in case of normally distributed data or as median (range) in case of not normally distributed data. Differences in continuous parameters were calculated with a student's $\mathrm{t}$-test when data were normally distributed and otherwise using a Mann-Whitney U test. BP = blood pressure; $\mathrm{BP}_{\text {intraop }}$ was analysed from time of anaesthesia induction until emergence.

\section{$\mathrm{BP}_{\text {intraop }}$ compared to $\mathrm{BP}_{\text {preind }}$}

Mean differences between $\mathrm{BP}_{\text {intraop }}$ and $\mathrm{BP}_{\text {preind }}$ were similar between DWI-positive and DWInegative patients for both systolic and diastolic BP (Table 3). In all patients relative hypotension occurred (median duration of 46 minutes; range 7 -133) with a comparable duration between the groups ( $p=.935)$. Intraoperative hypertension rarely occurred in both groups (Table 3 ).

\section{$\mathrm{BP}_{\text {intraop }}$ compared to $\mathrm{BP}_{\text {preop }}$}

When mean systolic $\mathrm{BP}_{\text {intraop }}$ was compared to systolic $\mathrm{BP}_{\text {preop }}$, DWI-positive patients had a larger decrease of $\mathrm{BP}_{\text {intraop }}(37 \pm 29 \mathrm{mmHg})$ compared to DWI-negative patients $(14 \pm 26 \mathrm{mmHg}$, $p=.024$ ). The difference in diastolic BP was not significantly different (Table 3). All eight DWIpositive patients had a lower mean systolic and/or diastolic $\mathrm{BP}_{\text {intraop }}$ compared to $\mathrm{BP}_{\text {preop }}$. Systolic relative hypotension, as compared to $\mathrm{BP}_{\text {preop}}$, endured a median of 32 (range 0-174) minutes in DWI-negative patients compared to 117 (range 29-169) minutes in DWI-positive patients $(p=.088)$. Differences between the groups were similar for diastolic $\operatorname{BP}(p=.071)$. All DWI-positive patients had an intraoperative period of relative hypotension of at least 30 minutes. Systolic hypertension, as compared to $\mathrm{BP}_{\text {intraop }}$, occurred with a median of 5 (range 0 -173) minutes in DWI-negative patients compared to 1 (range 0-15) in DWI-positive patients $(p=.048)$. 
Table 3 Results of intraoperative blood pressure measurements in relation to preoperative or preinduction blood pressure values of DWI-negative compared to DWI-positive patients

\begin{tabular}{|c|c|c|c|}
\hline A. $B P_{\text {intraop }}$ compared to $B P_{\text {preind }}$ & $\begin{array}{l}\text { DWI-negative } \\
(\mathrm{n}=47)\end{array}$ & $\begin{array}{l}\text { DWI -positive } \\
(n=8)\end{array}$ & $p$-value \\
\hline \multicolumn{4}{|l|}{ Mean differences } \\
\hline Mean systolic $\mathrm{BP}_{\text {intraop }}-$ systolic $\mathrm{BP}_{\text {preind }}$ in $\mathrm{mmHg}$ & $1 \pm 39$ & $-8 \pm 23$ & .558 \\
\hline Mean diastolic $\mathrm{BP}_{\text {intraop }}$ - diastolic $\mathrm{BP}_{\text {preind }}$ in $\mathrm{mmHg}$ & $-13 \pm 19$ & $-19 \pm 11$ & .370 \\
\hline \multicolumn{4}{|l|}{ Duration of relative hypotension } \\
\hline Systolic $\mathrm{BP}_{\text {intraop }}$ decrease $\geq 20 \%$ of $\mathrm{BP}_{\text {preind }}$ in minutes & $43(7-140)$ & $47(30-134)$ & .935 \\
\hline Diastolic $\mathrm{BP}_{\text {intraop }}$ decrease $\geq 20 \%$ of $\mathrm{BP}_{\text {preind }}$ in minutes & $111(36-209)$ & $139(91-169)$ & .173 \\
\hline \multicolumn{4}{|l|}{ Duration of relative hypertension } \\
\hline $\begin{array}{l}\text { Systolic } \mathrm{BP}_{\text {intraop }} \text { increase } \geq 20 \% \text { of } \mathrm{BP}_{\text {preind }} \\
\text { in minutes }\end{array}$ & $1(0-67)$ & $0(0-5)$ & .417 \\
\hline \multicolumn{4}{|l|}{ B. $B P_{\text {intraop }}$ compared to $B P_{\text {preop }}$} \\
\hline \multicolumn{4}{|l|}{ Mean differences } \\
\hline Mean systolic $\mathrm{BP}_{\text {intraop }}$ - systolic $\mathrm{BP}_{\text {preop }}$ in $\mathrm{mmHg}$ & $-14(26)$ & $-37(29)$ & .024 \\
\hline Mean diastolic $\mathrm{BP}_{\text {intraop }}$ - diastolic $\mathrm{BP}_{\text {preop }}$ in $\mathrm{mmHg}$ & $-19(14)$ & $-28(14)$ & .113 \\
\hline \multicolumn{4}{|l|}{ Duration of relative hypotension } \\
\hline Systolic $\mathrm{BP}_{\text {intraop }}$ decrease $\geq 20 \%$ of $\mathrm{BP}_{\text {preop }}$ in minutes & $32(0-174)$ & $69(15-140)$ & .088 \\
\hline Diastolic $\mathrm{BP}_{\text {intraop }}$ decrease $\geq 20 \%$ of $\mathrm{BP}_{\text {preop }}$ in minutes & $67(0-168)$ & $117(29-169)$ & .071 \\
\hline \multicolumn{4}{|l|}{ Duration of relative hypertension } \\
\hline $\begin{array}{l}\text { Systolic } \mathrm{BP}_{\text {intraop }} \text { increase } \geq 20 \% \text { of } \mathrm{BP}_{\text {preop }} \\
\text { in minutes }\end{array}$ & $5(0-173)$ & $1(0-15)$ & .048 \\
\hline
\end{tabular}

Data are given as mean \pm standard deviation (SD) in case of normally distributed data or as median (range) in case of not normally distributed data. Differences in continuous parameters were calculated with a student's $t$-test when data were normally distributed and otherwise using a Mann-Whitney $U$ test. BP = blood pressure; $\mathrm{BP}_{\text {intraop }}$ was analysed from time of anaesthesia induction until emergence, $\mathrm{BP}_{\text {preind }}$ was defined as an awake oscillometric non-invasive BP-measurement in the OR before administration of any medication used during induction of anaesthesia, $\mathrm{BP}_{\text {preop }}$ was defined as $\mathrm{BP}$ measured on the outpatient clinic or ward maximum 1 month before surgery. $\mathrm{BP}_{\text {intraop }}$ is expressed in relation to $\mathrm{BP}_{\text {preind }}(\mathrm{A})$ and in relation to $\mathrm{BP}_{\text {preop }}(\mathrm{B})$.

\section{$\mathrm{BP}_{\text {preind }}$ compared to $\mathrm{BP}_{\text {preop }}$}

Comparison of $\mathrm{BP}_{\text {preind }}$ with $\mathrm{BP}_{\text {preop }}$ did not results in any significant differences between DWInegative and DWI-positive patients $(6 \pm 18 \mathrm{mmHg}$ vs $2 \pm 7 \mathrm{mmHgs}$ respectively, $p=.535)$

\section{Secondary outcome measures}

DWI lesion volume and count were both significantly correlated to systolic $\mathrm{BP}_{\text {preop }}\left(\mathrm{r}^{2}=.336\right.$, $p=.012$ and $r^{2}=.371, p=0.005$ respectively) and mean differences between $\mathrm{BP}_{\text {intraop }}$ and $\mathrm{BP}_{\text {preop }}$ $\left(r^{2}=-.296, p=.028\right.$ and $r^{2}=-.305, p=.025$ respectively). Other perioperative blood pressure values or duration of hypotension was not significantly correlated to DWI lesion volume and count (see supplemental data). Intraoperative use of vasoactive medication varied between the patients but all but one patient received phenylephrine during surgery. Maximum dosage of phenylephrine was higher in DWI-positive compared to DWI-negative patients $(25.1 \pm 8.06 \mathrm{vs}$ $17.0 \pm 7.07 \cdot 10^{2} 100 \mu \mathrm{g} / \mathrm{ml} p=0.005$ ). Ephedrine and atropine were used in 21 and 11 patients 
respectively, dosages did not differ between DWI-positive and DWI negative patients. Selective shunting based on EEG changes was indicated and used in five patients; two of them were DWI-positive. Sensitivity analysis excluding shunted patients did not affect the abovementioned results (data not shown). 42/55 patients (76\%) had intraoperative TCD data available (see supplemental data). Mean flow velocity of the ipsilateral MCA during cross-clamping had changed with a median of $-21 \%$ (range $-100 \%-59 \%$ ) in DWI-negative patients versus $-30 \%$ $(-100 \%-38 \%)$ in DWI-positive compared to velocity prior to cross-clamping ( $p=.426)$. Change of mean flow velocity on the contralateral side was $15 \%$ (range $-10 \%-94 \%$ ) in DWI-negative patients versus $27 \%(7 \%-80 \%)$ in DWI-positive patients ( $p=.086)$. Micro emboli were detected in 16/37 (43\%) DWI-negative versus 4/5 (80\%) DWI-positive patients ( $p=.122)$. Arteriotomy was closed by venous patch in 44 patients $(80 \%)$, Dacron patch in 4 patients $(7.3 \%)$, bovine patch in 1 patient (1.8\%) and primary closed in 6 patients (10.9\%). Six patients had a suspicion of cerebral hyperperfusion based on increased MCA flow velocity or increase in BP (all DWInegative patients). Four of these patients developed cerebral hyperperfusion syndrome during hospital admission, of which one was complicated by intracerebral haemorrhage. The median follow-up period was three years. Of 47 DWI-negative patients, four developed a stroke of which one ischaemic after 1.5 year and three haemorrhagic (two in perioperative period and one six months after CEA). One additional patient died of cardiovascular origin one year after CEA (sudden cardiac death). Of patients with new postoperative DWI lesions, one suffered from ischaemic stroke in the perioperative period.

\section{Discussion}

In this study, we explored the association between intraoperative BP characteristics and new silent brain ischaemia as documented with DWI. Our preliminary results suggest that DWIpositive patients have a higher systolic and diastolic $\mathrm{BP}_{\text {preop }}$ and that drop of systolic $\mathrm{BP}_{\text {intraop }}$ is larger than in DWI-negative patients. Without exception, all DWI-positive patients had a lower $\mathrm{BP}_{\text {intraop }}$ compared to $\mathrm{BP}_{\text {preop }}$ and all DWI-positive patients experienced at least 30 minutes of intraoperative relative hypotension. We also found that these differences were only visible when intraoperative measurements were compared to preoperative baseline values and not when compared to BP just prior to induction. Absolute values of intraoperative BP did not differ between the groups.

These results may suggest that intraoperative relative hypotension contributes to the development of silent brain ischaemia. This is in accordance with earlier literature suggesting that haemodynamic events after CEA increase the risk of ischaemic lesions ${ }^{19}$. Nevertheless, this previous study investigated major haemodynamic events (bradycardia $<40 \mathrm{bpm}$, asystole or hypotension requiring treatment), and it was unclear how often hypotension occurred in these patients and how severe these hypotensive episodes were. It is not surprising that such extreme haemodynamic disturbances result in brain ischaemia as cerebral perfusion pressure (CPP) may fall outside the range of autoregulatory mechanisms. The thirteen patients included in our study that showed a period of absolute hypotension $(<80 \mathrm{mmHg})$ only had this for a very 
short time (maximum of 7 minutes). This may be the reason that this hypotensive episode did not result in any brain ischaemia in these patients. Our study focused mainly on more subtle variations in intraoperative BP since little is known on which intraoperative target BP to strive for and how rigorous this should be maintained. One other study that randomized patients between normotension and hypertension ( $>110 \%$ of baseline BP) during CEA found that the hypertension group had a decreased chance of new ischaemic lesions ${ }^{9}$ which is supported by our results showing that DWI-negative patients had a systolic BP $>120 \%$ of baseline for a longer time compared to DWI-positive patients.

The difference between mean $\mathrm{BP}_{\text {intraop }}$ and baseline was only present when compared to $\mathrm{BP}_{\text {preop }}$ (and not to BP just prior to induction). All but one DWI-positive patient had a $\mathrm{BP}_{\text {preop }}$ of at least $170 \mathrm{mmHg}$. The difference between $\mathrm{BP}_{\text {preop }}$ and $\mathrm{BP}_{\text {preind }}$ may be explained by the fact that a subgroup of patients was not adequately treated for hypertension or was not compliant to their prescribed medication when first admitted to the hospital. This is consistent with the fact that although a large difference in baseline BP is present between the groups, reported hypertension or use of anti-hypertensive medication did not differ, although reported use of medication may be higher than actual use due to compliance issues. Blood pressure lowering therapy initiated or restarted upon hospital admission may have decreased the BP in this subgroup to a value lower than the patient was accustomed to. $\mathrm{BP}_{\text {preind }}$ may therefore not be the most ideal BP to use as a baseline to set intraoperative target values ${ }^{24}$, and although $\mathrm{BP}_{\text {preop }}$ measurements were not standardized in our study, they may represent a better reflection of a patient's true BP. These results also contribute to the discussion of whether or not to perform aggressive lowering of BP in the period before revascularization ${ }^{25}$. Although systolic $\mathrm{BP}>180 \mathrm{mmHg}$ has been independently associated with an increased risk of stroke after $\mathrm{CEA}^{26}$, is it unclear whether lowering this $\mathrm{BP}$ in the preoperative period reduces the risk of events.

Possibly, patients with high baseline BP are at risk of brain ischaemia because of failure of autoregulatory mechanisms. Under physiological conditions, cerebral blood flow (CBF) is generally unaffected by changes in cerebral perfusion pressure (CPP), which is the difference between the MAP and intracranial pressure. If CPP is outside its normal range, reflex vasodilation occurs to maintain CBF due to cerebral autoregulation ${ }^{27}$. Studies have previously shown that CAD patients may have impaired cerebral autoregulation ${ }^{5}$ and additionally have attenuated baroreflex sensitivity as a result of reduced distensibility of the carotid bulbs infiltrated by atherosclerosis ${ }^{6}$. During cross-clamping of the carotid artery, ipsilateral CPP may be reduced and baroreceptor sensitivity may be further compromised by surgical trauma to sensory nerves within the arterial wall ${ }^{3}$. We hypothesize that ischaemic brain lesions may develop in patients that have a diminished baroreceptor sensitivity for (relatively) low arterial pressure, perhaps in combination with an impaired cerebral autoregulation. Patients with pre-existing untreated or inadequately treated hypertension may be particularly susceptible to perioperative haemodynamic complications as their baroreceptors may be even more affected due to continuous exposure to high $\mathrm{BP}^{28}$. This theory is supported by the observation that a higher maximum dose of vasoactive medication was used in the DWI-positive group, suggesting that in these patients maintaining adequate blood pressure is more challenging. 
All patients had cerebral monitoring by EEG and TCD and selective shunting was used. There were no differences between the groups in monitoring results and shunt use. This suggests that possibly differences in CBF resulting in DWI lesions may not be prominent enough to be detected by cerebral monitoring techniques.

\section{Future perspectives}

Our preliminary results suggest that suboptimal perioperative haemodynamics may contribute to the development of cerebral complications after CEA. However, no strong conclusions can be drawn on what the true cause of the increased risk of DWI lesions is; either high preoperative BP or relatively low intraoperative BP. We do believe that perioperative blood pressure management should focus on maintaining adequate CPP during crossclamping despite complicating factors such as impaired collateral circulation and decreased baroreceptor sensibility. Aggressive reduction of BP during the time interval between hospital presentation and surgery may unjustly lead to a too low target BP during surgery in patients with already impaired autoregulatory mechanisms. The optimal strategy for preoperative normalization of hypertensive BP should be a topic for further research. This should also include the consideration whether or not to omit anti-hypertensive medication on the morning of surgery. Additionally, a trial randomizing between high versus low intraoperative target BP like previously performed in patients undergoing cardiac surgery ${ }^{29}$, can be helpful for determining optimal intraoperative BP management in CEA patients.

\section{Limitations}

We are aware of the chance of type II statistical error or overfitting of our statistical model as a result of the small patient cohort. We were only able to include a limited number of patients in this study due to its retrospective design. Although there are some differences detected in $\mathrm{BP}_{\text {intraop }}$ between the two groups, many of the investigated parameters approached but did not reach statistical significance. Correlation between systolic $\mathrm{BP}_{\text {preop }}$ and postoperative DWI lesion volume and count was significant but weak. Nonetheless, the investigated features all show the same direction of effect between DWI-negative and DWI-positive patients with a difference between the groups that seems clinically relevant. Furthermore, the detailed information on pre- and intraoperative BP that was available in this exploratory study provides unique inside in perioperative haemodynamics and its relation to brain ischaemia on MR-DWI.

Because of the limited number of events, we deliberately chose not to correct for potential confounders. Yet, baseline variables (except for BP at hospital intake) were very similar between the groups. We cannot distinguish whether relatively low $\mathrm{BP}_{\text {intraop }}$ alone or other mechanisms resulting from a high baseline BP contribute to the risk of DWI lesions. Correcting for baseline BP in our analysis would eliminate the effect of $\mathrm{BP}_{\text {intraop }}$ on the outcome because of their interaction.

Earlier literature already described high $\mathrm{BP}_{\text {preop }}$, both systolic and diastolic, to be an independent predictor for periprocedural events ${ }^{30,31}$. These studies also suggest that this correlation may be a result of intraoperative impairment of CBF due to failed autoregulatory mechanisms. However, more research in larger patient cohorts, stratifying for differences in pre- and intraoperative characteristics, 
is warranted to identify factors that increase the risk of brain ischaemia. TCD data was only available in a limited number of patients. As such no conclusions can be drawn on presence or absence of any association between TCD measurements and intraoperative blood pressure or DWI lesions. $\mathrm{BP}_{\text {preop }}$ was adopted from the patient's medical file and measurements used for further analysis were not standardized in time of measurement. $\mathrm{BP}_{\text {preop }}$ was measured using NIBP, while invasive radial artery $\mathrm{BP}$-measurements were used for $\mathrm{BP}_{\text {intraop }}$. Additionally, high BP's measured at intake or hospital admission may sometimes be due to white coat hypertension ${ }^{32}$. Last, in this study only relative intraoperative hypertension (defined as $>20 \%$ above baseline) was scored. No absolute threshold for intraoperative hypertension was defined, as threshold described in literature are ambiguous and vary from $140 \mathrm{mmHg}$ to $>180 \mathrm{mmHg}$. Furthermore, relative intraoperative hypertension is more patient specific $^{33}$. Although these are limitations of our study design, our results can be easily translated to clinical practice.

\section{Conclusion}

In this exploratory study high preoperative BP and a larger drop of intraoperative BP are associated with the occurrence of periprocedural cerebral ischaemia. Patients with untreated or inadequately treated hypertension might be more prone to suffer from cerebral haemodynamic disturbances due to a relatively low intraoperative BP and may therefore be at risk for development of ischaemic lesions. We are in need of prospective studies that investigate pre- and intraoperative BP and its relation to ischaemic brain lesions in larger cohorts. 


\section{References}

1. Huibers A, Calvet D, Kennedy F, Czuriga-Kovács KR, Featherstone RL, Moll FL, et al. Mechanism of Procedural Stroke Following Carotid Endarterectomy or Carotid Artery Stenting Within the International Carotid Stenting Study (ICSS) Randomised Trial. Eur J Vasc Endovasc Surg. 2015;50(3):281-8.

2. Huibers A, De Borst GJ, Thomas DJ, Moll FL, Bulbulia R, Halliday A. The mechanism of procedural stroke following carotid endarterectomy within the asymptomatic carotid surgery trial 1. Cerebrovasc Dis. 2016;42(3-4):178-85.

3. Landesberg G, Adam D, Berlatzky Y, Akselrod S. Step baroreflex response in awake patients undergoing carotid surgery: time- and frequency-domain analysis. Am J Physiol Circ Physiol. 2017;274(5):H1590-7.

4. Romero JR, Pikula A, Nguyen TN, Nien YL, Norbash A, Babikian VL. Cerebral Collateral Circulation in Carotid Artery Disease. Curr Cardiol Rev. 2010;5(4):279-88.

5. Powers WJ. Cerebral hemodynamics in ischemic cerebrovascular disease. Ann Neurol. 1991;29(3):231-40.

6. Nasr N, Czosnyka M, Pavy-Le Traon A, Custaud M-A, Liu X, Varsos G V, et al. Baroreflex and cerebral autoregulation are inversely correlated. Circ J. 2014;78(10):2460-7.

7. Caplan LR, Hennerici M. Impaired clearance of emboli (washout) is an important link between hypoperfusion, embolism, and ischemic stroke. Arch Neurol. 1998;55(11):1475-82.

8. Fujimoto K, Matsumoto Y, Oikawa K, Nomura JI, Shimada Y, Fujiwara S, et al. Cerebral hyperperfusion after revascularization inhibits development of cerebral ischemic lesions due to artery-to-artery emboli during carotid exposure in endarterectomy for patients with preoperative cerebral hemodynamic insufficiency: revisiting the "impaired clearance of emboli" concept. Int J Mol Sci. 2016;17(8).

9. Kobayashi M, Ogasawara K, Yoshida K, Sasaki M, Kuroda H, Suzuki T, et al. Intentional hypertension during dissection of carotid arteries in endarterectomy prevents postoperative development of new cerebral ischemic lesions caused by intraoperative microemboli. Neurosurgery. 2011 Aug;69(2):301-7.

10. Gensicke H, Van Der Worp HB, Nederkoorn PJ, Macdonald S, Gaines PA, Van Der Lugt A, et al. Ischemic brain lesions after carotid artery stenting increase future cerebrovascular risk. J Am Coll Cardiol. 2015;65(6):521-9.

11. Schnaudigel S, Gröschel K, Pilgram SM, Kastrup A. New brain lesions after carotid stenting versus carotid endarterectomy: a systematic review of the literature. Stroke. 2008;39(6):1911-9.

12. Gensicke H, Zumbrunn T, Jongen LM, Nederkoorn PJ, MacDonald S, Gaines PA, et al. Characteristics of ischemic brain lesions after stenting or endarterectomy for symptomatic carotid artery stenosis: Results from the international carotid stenting study-magnetic resonance imaging substudy. Stroke. 2013;44(1):80-6.

13. Ederle J, Dobson J, Featherstone RL, Bonati LH, van der Worp HB, de Borst GJ, et al. Carotid artery stenting compared with endarterectomy in patients with symptomatic carotid stenosis (International Carotid Stenting Study): an interim analysis of a randomised controlled trial. Lancet. 2010;375(9719):985-97.

14. Bonati LH, Jongen LM, Haller S, Flach HZ, Dobson J, Nederkoorn PJ, et al. New ischaemic brain lesions on MRI after stenting or endarterectomy for symptomatic carotid stenosis: a substudy of the International Carotid Stenting Study (ICSS). Lancet Neurol. 2010;9(4):353-62.

15. Traenka C, Engelter ST, Brown MM, Dobson J, Frost C, Bonati LH. Silent brain infarcts on diffusionweighted imaging after carotid revascularisation: A surrogate outcome measure for procedural stroke? A systematic review and meta-analysis. Eur Stroke J. 2019;4(2):127-43.

16. Mrkobrada M, Chan MTV, Cowan D, Campbell D, Wang CY, Torres D, et al. Perioperative covert stroke in patients undergoing non-cardiac surgery (NeuroVISION): a prospective cohort study. Lancet. 2019;394:1022-9.

17. Naylor AR, Spence JD. Adding Insight to Injury. Eur J Vasc Endovasc Surg. 2019; In press.

18. Lei C, Deng Q, Li H, Zhong L. Association Between Silent Brain Infarcts and Cognitive Function: A Systematic Review and Meta-Analysis. J Stroke Cerebrovasc Dis. 2019;28(9):2376-87.

19. Altinbas A, Algra A, Bonati LH, Brown MM, Kappelle LJ, De Borst GJ, et al. Periprocedural hemodynamic depression is associated with a higher number of new ischemic brain lesions after stenting in the international carotid stenting study-MRI substudy. Stroke. 2014;45(1):146-51.

20. Ito $\mathrm{Y}$, Kato N, Matsumura A, Sonobe M. Hemodynamic instability increases new ischemic brain lesions on diffusion-weighted imaging after carotid artery stenting. Neurol Med Chir (Tokyo). 2013;53(6):375-80.

21. Ten Berg MJ, Huisman A, Van Den Bemt PMLA, Schobben AFAM, Egberts ACG, Van Solinge WW. Linking laboratory and medication data: New opportunities for pharmacoepidemiological research. Clin Chem Lab Med. 2007;45(1):13-9.

22. Bijker B, Klei van W, Kappen T, Wolfswinkel van L, Moons K, Kalkman C. Incidence of Intraoperative Hypotension as a Function of the Chosen Definition. Surv Anesthesiol. 2008;52(5):237-8.

23. Sabljak VD, Zivaljevic VR, Milicic BR, Paunovic IR, Toskovic AR, Stevanovic KS, et al. Risk Factors for Intraoperative Hypertension during Surgery for Primary Hyperparathyroidism. Med Princ Pract. 2017;26(4):381-6. 
24. van Klei WA, van Waes JAR, Pasma W, Kappen TH, van Wolfswinkel L, Peelen LM, et al. Relationship Between Preoperative Evaluation Blood Pressure and Preinduction Blood Pressure. Anesth Analg. 2017;124(2):431-7.

25. Naylor AR, Ricco JB, de Borst GJ, Debus S, de Haro J, Halliday A, et al. Management of Atherosclerotic Carotid and Vertebral Artery Disease: 2017 Clinical Practice Guidelines of the European Society for Vascular Surgery (ESVS). Eur J Vasc Endovasc Surg. 2018;55(1):3-81.

26. Bond R, Narayan SK, Rothwell PM, Warlow CP. Clinical and radiographic risk factors for operative stroke and death in the European Carotid Surgery Trial. Eur J Vasc Endovasc Surg. 2002;23(2):108-16.

27. Sigaudo-Roussel D, Evans DH, Naylor AR, Panerai RB, London NL, Bell P, et al. Deterioration in carotid baroreflex during carotid endarterectomy. J Vasc Surg. 2002;36(4):793-8.

28. Piccirillo G, Di Giuseppe V, Nocco M, Lionetti M, MoisÃ" A, Naso C, et al. Influence of Aging and Other Cardiovascular Risk Factors on Baroreflex Sensitivity. J Am Geriatr Soc. 2002;49(8):1059-65.

29. Vedel AG, Holmgaard F, Rasmussen LS, Langkilde A, Paulson OB, Lange T, et al. High-target versus lowtarget blood pressure management during cardiopulmonary bypass to prevent cerebral injury in cardiac surgery patients: A randomized controlled trial. Circulation. 2018;137(17):1770-80.

30. Doig D, Turner EL, Dobson J, Featherstone RL, De Borst GJ, Stansby G, et al. Risk Factors for Stroke, Myocardial Infarction, or Death Following Carotid Endarterectomy: Results from the International Carotid Stenting Study. Eur J Vasc Endovasc Surg. 2015;50(6):688-94.

31. de Waard DD, de Borst GJ, Bulbulia R, Huibers A, Halliday A. Diastolic Blood Pressure is a Risk Factor for Peri-procedural Stroke Following Carotid Endarterectomy in Asymptomatic Patients. Eur J Vasc Endovasc Surg. 2017;53(5):626-31.

32. Verberk WJ, Kroon AA, Thien T, Lenders JWM, Van Montfrans GA, Smit AJ, et al. Prevalence of the white-coat effect at multiple visits before and during treatment. J Hypertens. 2006;24(12):2357-63

33. Williams B, Mancia G, Spiering W, Rosei EA, Azizi M, Burnier M, et al. 2018 ESC/ESHGuidelines for themanagement of arterial hypertension. Eur Heart J. 2018;39:3021-104. 
Intraoperative hypotension during CEA 


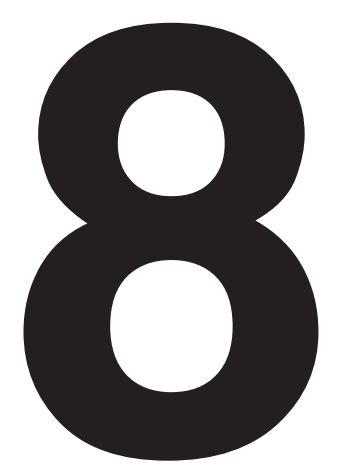

\section{Effect of bilateral carotid occlusion on cerebral haemodynamics and perivascular innervation: An experimental rat model}

ML Rots, GJ de Borst, A van der Toorn, FL Moll, CWA Pennekamp, RM Dijkhuizen,

RLAW Bleys

J Comp Neurol 2019; 527 (14): 2263-2272 


\section{Abstract}

We aimed to investigate the effect of chronic cerebral hypoperfusion on cerebral haemodynamics and perivascular nerve density in a rat model. Bilateral common carotid artery $(C C A)$ ligation $(n=24)$ or sham-operation $(n=24)$ was performed with a one-week interval. A subgroup (ligated $n=6$; sham-operated $n=3$ ) underwent magnetic resonance imaging (MRI) before the procedures and two and four weeks after the second procedure. After termination, carotids were harvested for assessment of complete ligation and nerve density in cerebral arteries that were stained for the general neural marker PGP 9.5 and sympathetic marker TH by computerized image analysis. Five rats were excluded because of incomplete ligation. MRI-based tortuosity of the posterior communicating artery (Pcom), first part of the posterior cerebral artery (P1) and basilar artery was observed in the ligated group, as well as an increased volume $(p=0.05)$ and relative signal intensity in the basilar artery $(p=0.04)$ (shamgroup unchanged). Immunohistochemical analysis revealed that compared to sham-operated rats, ligated rats had increased diameters of all intracircular segments and the extracircular part of the internal carotid artery $(p<0.05)$. Ligated rats showed a higher general nerve density compared to controls in $\mathrm{P} 1$ (10\%, IQR:8.7-10.5 versus 6.6\%, IQR:5.5-7.4, $\mathrm{p}=0.003$ ) and Pcom segments (6.4\%, IQR:5.8-6.5 versus $3.2 \%$, IQR:2.4-4.3, $\mathrm{p}=0.003)$ and higher sympathetic nerve density in Pcom segments (3.7\%, IQR:2.8-4.8 versus 1.7\%, IQR:1.3-2.2, $\mathrm{p}=0.02$ ). Bilateral CCA occlusion resulted in redistribution of blood flow to posteriorly located cerebral arteries with remarkable changes in morphology and perivascular nerve density, suggesting a functional role for perivascular nerves in cerebral autoregulation. 


\section{Introduction}

Cerebral hypoperfusion due to significant steno-occlusive disease has been shown to play an important role in the occurrence of cerebral ischaemia'. The haemodynamic effect of a stenosis in the common carotid artery (CCA) on perfusion of the brain tissue depends mainly on the adequacy of collateral circulatory pathways ${ }^{2,3}$. The most important collaterals are formed by the circle of Willis, which connects the internal carotid arteries and the vertebrobasilar system. The functional role of the circle of Willis in serving as a collateral to sustain adequate cerebral blood flow (CBF) during and after carotid surgery however is still unpredictable. The CBF is dependent on the cerebral perfusion pressure, the diameter of cerebral blood vessels, the viscosity of the blood, as well as factors affecting these parameters ${ }^{4}$. As a result of cerebral autoregulation, CBF is generally unaffected by changes in cerebral perfusion pressure (CPP; difference between the mean arterial pressure and intracranial pressure) provided that CPP is within normal levels $(\sim 60$ to $160 \mathrm{mmHg})$. When CPP is seriously affected and falls outside this range, $\mathrm{CBF}$ is directly dependent on $\mathrm{CPP}^{5,6}$. The exact physiology of these autoregulatory mechanisms has not yet been clarified, but the following mechanisms are thought to interact and contribute to the process of cerebral autoregulation: 1) metabolic or chemical mechanisms: vessel diameter and vascular resistance are affected by the concentration of vasoactive substances involved in tissue metabolism (e.g. $\mathrm{O}_{2}, \mathrm{CO}_{2}, \mathrm{H}^{+}$), 2) myogenic mechanism: the capacity of vascular smooth muscle to constrict in response to a transmural pressure increase, and 3) neurogenic mechanism: cerebrovascular innervation may be involved in controlling cerebral haemodynamics ${ }^{5}$. The dense nerve supply to the major cerebral arteries consists of sympathetic, parasympathetic, and sensory fibers ${ }^{7}$. The regulatory role of sympathetic (vasoconstriction) and parasympathetic (vasodilation) nerve fibers manifests itself by a topographical heterogeneity of nerve densities, corresponding to the potential fluctuations in flow to the concerning brain segment. Perivascular sympathetic innervation has also been demonstrated to induce hypoperfusion after global cerebral ischaemia, whereas parasympathetic innervation may play a role in cerebral protection under ischaemic circumstances ${ }^{7,8}$. Importantly, the nerve density has been shown to be dynamic with the capacity to adapt to altering functional demands ${ }^{9}$. For example, a decreased metabolic activity induced by anosmia resulted in a decreased overall nerve density in the anterior cerebral artery, the main arterial supplier of the rhinencephalon ${ }^{10}$. Furthermore, a reduction in cerebral blood flow occurs in both rats and humans due to aging and Alzheimer's disease respectively and have been correlated to a decline in overall and sympathetic nerve density in the anterior cerebral artery ${ }^{11,12}$.

Based on the observation of adapting periarterial nerve density, we hypothesized that permanent bilateral CCA occlusion in rats, will result in local haemodynamic changes and subsequently induce alterations in cerebral artery nerve density. Therefore, the purpose of this study was to evaluate the effects of a staged bilateral CCA occlusion, using an established animal model to study the effect of chronic cerebral hypoperfusion-related changes, on cerebral haemodynamics and nerve density of the basal cerebral arteries in rats ${ }^{13}$. 


\section{Materials and Methods}

\section{Rat ligation model}

All animal procedures were carried out under protocols approved by the Animal Experiments Committee of the University Medical Center Utrecht and Utrecht University and in accordance with the guidelines of the European Communities Council Directive.

Forty-eight Crl:CD (Sprague Dawley) rats weighing 300 to 350 gram (Charles River Laboratories, Wilmington, MA, RRID:RGD_734476) were included in the study. Rats were randomly assigned to either bilateral CCA ligation procedure with a one-week interval between the first and the second CCA occlusion ${ }^{14}$ or sham operation (Figure 1).

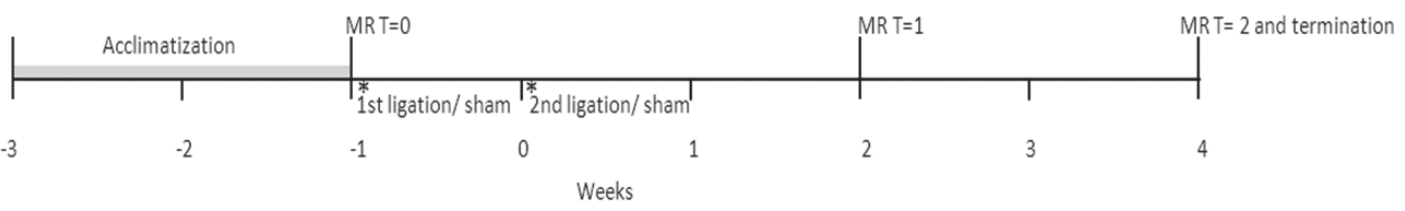

Figure 1 Timeline. $t=0$ : preoperative, $t=1$ : two weeks after bilateral ligation and $t=2$ : four weeks after bilateral ligation.

Both the sham-operated and ligated animals were sacrificed and processed for immunohistochemical studies at 28 days after the second surgical procedure ${ }^{10}$. All procedures were performed between October 2012 and February 2013 in the morning in the operating rooms of the Central Laboratory Animal Research Facility Utrecht. For ligation or shamoperation animals were pre-medicated with buprenorphine $(0.05 \mathrm{mg} / \mathrm{kg}$ i.m.), anaesthetized by mechanical ventilation with $4 \%$ isoflurane in air (induction) and $2 \%$ isoflurane (maintenance). Blood oxygen saturation was continuously monitored during surgical procedures. After shaving and disinfection of the ventral surface of the neck with iodine rats were placed on a heating pad. Rats were randomly assigned to first left-sided or first right-sided operation. Under an operation microscope a midline ventral incision of $6 \mathrm{~cm}$ was made with blade no. 15 and the unilateral carotid artery was bluntly separated from the cervical sympathetic trunk and the vagus nerve. The CCA was subsequently ligated with a 6-0 silk suture. In the 24 sham-operated rats the CCA was only exposed, but not ligated.. After haemostasis intracutaneous wound closure with Vicryl 4-0 was performed. One week after one-sided operation, the contralateral CCA was operated using the primary incision and access route, resulting in 24 rats with bilateral ligation and 24 rats with a bilateral sham procedure.

All procedures were performed after an acclimatization period of two weeks. All animals were group-housed under standard, controlled conditions (food and water provided ad libitum, room temperature 22-24 C, $12 \mathrm{~h}$ light/12 h dark cycle). An entry control with weighing and controls was performed by an animal keeper. Postoperatively welfare and weight were assessed daily during the first two days and weekly during the remaining study period. 


\section{Magnetic Resonance Imaging (MRI)}

Magnetic Resonance (MR) imaging of the brain in a 4.7 T animal MR system (Agilent, Palo Alto, CA, USA) in the first consecutive nine rats (MR-group: ligated $n=6$ and sham-operated $\mathrm{n}=3$ ) was performed to evaluate the effect of bilateral ligation on cerebral haemodynamics at three timepoints: $t=0$ (before ligation), $t=1$ (two weeks after bilateral ligation) and $t=2$ (four weeks after bilateral ligation, just before sacrifice). See Figure 1. Anaesthesia was induced with $4 \%$ isoflurane in air followed by administration of $5 \mathrm{ml} \mathrm{NaCl} 0.9 \%$ subcutaneously. During MR scanning the rats were mechanically ventilated with $2 \%$ isoflurane in air. The animals were restrained in the scanner with earplugs and a tooth-holder. Body temperature was measured with a rectal probe and was kept at $37^{\circ} \mathrm{C}$ with a heating pad during the imaging procedure. A home-built Helmholtz volume coil (i.d. $90 \mathrm{~mm}$ ) was used for signal excitation and a $35 \mathrm{~mm}$ inductively coupled surface coil was used for signal reception.

To evaluate whether either changes in volume or relative signal intensity of the basilar artery occurred, two flow-compensated 3D time-of-flight MR angiograms (MRA) of the basal cerebral arteries were acquired (repetition time (TR)/echo time (TE) $20 / 2 \mathrm{~ms}$, flip angle $40^{\circ}$, field-ofview (FOV) $20 \times 25.2 \times 15.2 \mathrm{~mm}^{3}$, data matrix size $100 \times 126 \times 76$ points, 4 averages). The second angiogram was acquired $7.5 \mathrm{~mm}$ posterior to the first one. Additionally, to determine whether the bilateral occlusion caused a reduced perfusion of the brain, MRI with arterial spin labeling (ASL) was performed using Flow-Sensitive Alternating Inversion Recovery (FAIR) $)^{15,16}$ combined with a 2-shot gradient-echo EPI acquisition for image read-out (TR/TE 10000/4.8 $\mathrm{ms}$, delay between images in the inversion curve $150 \mathrm{~ms}$, flip angle $10^{\circ}$, FOV $32 \times 32 \mathrm{~mm}^{2}$, data matrix $64 \times 64$, slice thickness $2 \mathrm{~mm}$, selective inversion slab: $10 \mathrm{~mm}, 16$ averages). Blood oxygenation level-dependent (BOLD)-MRI with carbogen $\left(5 \% \mathrm{CO}_{2}, 95 \% \mathrm{O}_{2}\right)$ inhalation as a hypercapnic stimulus was acquired to assess cerebrovascular reactivity. The BOLD acquisition protocol consisted of a 2D single shot spin-echo EPI acquisition (TR/TE 2000/27 $\mathrm{ms}$, FOV $32 \times 32 \mathrm{~mm}^{2}$, data matrix size $64 \times 64$ points, 24 coronal slices of $0.5 \mathrm{~mm}$ thickness) repeated 600 times. During the first 150 acquisitions the rat was ventilated with an air: $\mathrm{O}_{2}(2: 1)$ mixture, followed by 150 acquisitions with $100 \% \mathrm{O}_{2}$. The third set of 150 acquisitions was performed during ventilation with carbogen, and this was followed by 150 acquisitions during ventilation with the air: $\mathrm{O}_{2}$ mixture again.

\section{Tissue preparation}

Four weeks after the second operation the rats were terminated by perfusion fixation with a Watson Marlow SCI 323 peristaltic pump (Watson-Marlow Pumps Group, Falmouth, Cornwall, UK). Under anesthesia (sodium pentobarbital $0.1 \mathrm{ml} / 100$ gram body weight i.p.) the ascending aorta was cannulated and the rats were perfused with $300 \mathrm{ml} 0.9 \% \mathrm{NaCl}$ containing 5000 IE heparin, $500 \mathrm{ml} 4 \%$ paraformaldehyde in $0.1 \mathrm{M}$ phosphate buffer $\left(\mathrm{pH} 7.4\right.$, at $\left.4^{\circ} \mathrm{C}\right)$ and $500 \mathrm{ml}$ $15 \%$ sucrose in $0.1 \mathrm{M}$ phosphate buffer $\left(\mathrm{pH} 7.4 \text {, at } 4^{\circ} \mathrm{C}\right)^{10}$. Subsequently, the brains including basal cerebral arteries were removed and stored in $15 \%$ sucrose in $0.1 \mathrm{M}$ phosphate buffer $\left(\mathrm{pH} 7.4\right.$, at $\left.4^{\circ} \mathrm{C}\right)$. 


\section{Immunohistochemistry}

After termination all subjects were macroscopically evaluated for successful carotid ligation. If the ligation was questionable based on both in vivo MR angiograms and post mortem macroscopical evaluation (MR-group) or on post mortem macroscopical evaluation only (nonMR group) these rats were excluded from further analysis. The basal cerebral arteries were dissected of the brain, divided in segments (Figure 2) and mounted on Sylgard (Dow Corning, Midland, MI USA) with entomology needles. Subsequently, the ligated group $(n=24)$ and shamoperated group ( $n=24)$ were divided into three subgroups (each $n=8$ ), which underwent various immunohistochemical procedures to localize the general neural marker protein gene product 9.5 (PGP 9.5; ligated $n=8$ and sham-operated $n=8$ ), the parasympathetic nerves containing vesicular acetylcholine transporter (VAChT; ligated $n=8$ and sham-operated $n=8$ ), or the sympathetic nerves containing tyrosine hydroxylase $(\mathrm{TH}$; ligated $\mathrm{n}=8$ and sham-operated $n=8)$.

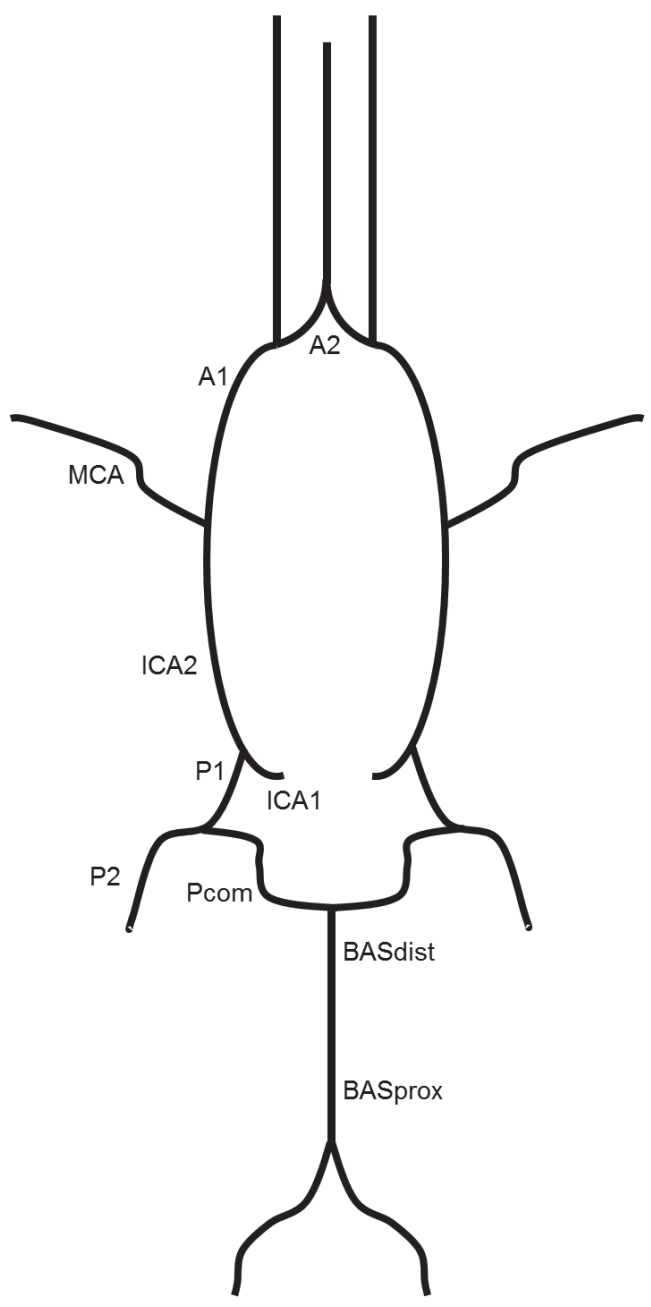

Figure 2 Schematic overview of the segments of the circle of Willis. A1: first part of the anterior cerebral artery, A2: second part of the anterior cerebral artery, ICA1: first part of the internal carotid artery, ICA2: second part of the internal carotid artery, MCA: middle cerebral artery, P1: first part of the posterior cerebral artery, P2: second part of the posterior cerebral artery, Pcom: posterior communicating artery, BASprox: proximal part of the basilar artery and BASdist: distal part of the basilar artery. 
The segments that underwent PGP 9.5 staining were washed three times in Hepes buffer containing $0.1 \%$ Triton $X-100$ for 10 minutes followed by incubation of $5 \%$ normal swine serum (Jackson immunoresearch, West Grove, PA, USA) in Hepes buffer containing 0.1\% Triton X-100 for 90 minutes. Subsequently, they were incubated in Rabbit anti-PGP 9.5 (Millipore Cat\# AB5925, RRID:AB-92138)) diluted 1:400 in Hepes buffer containing 0.1\% Triton X-100, 0.1\% DL lysine and $1 \%$ normal swine serum for 48 hours at $4^{\circ} \mathrm{C}$. After two days the segments were washed three times in PBS for 10 minutes and the segments were incubated in fluorescein isothiocyanate (FITC)- conjugated swine anti-Rabbit serum (Dako, Denmark) diluted in 1:40 in PBS containing 1\% normal swine serum, 0.1\% Triton X-100 and 0.1\% DL-lysine for 90 minutes. Thereafter, the segments were washed three times in PBS for 10 minutes and stained for 10 minutes in $0.05 \%$ pontamine sky blue (Gurr, Poole, UK) to reduce background autofluorescence and washed in PBS three times for five minutes. ${ }^{17}$ Lastly, the segments were stretched on glass slides and mounted with Vectashield (Vector Laboratories, Inc., Burlingame, CA) mounting medium. Segments that underwent VAChT staining were washed three times in Hepes buffer containing $0.1 \%$ Triton $X-100$ for 10 minutes followed by incubation of $5 \%$ normal goat serum (Jackson immunoresearch, West Grove, PA, USA) in Hepes buffer containing $0.1 \%$ Trion $\mathrm{X}-100$ for 90 minutes. The segments were subsequently incubated overnight at room temperature in Rabbit anti-VAChT (Abcam CAT\# ab68984, RRID:AB-1143825) diluted 1:1500 in Hepes buffer containing 0.1\% Triton X-100, 0.1\% DL lysine, 1\% normal goat serum and 5\% Bovine Serum Albumin (Across Organics, Fair Lawn, New Jersey, USA) After washing three times in PBS for 10 minutes the segments were incubated in Streptavidin FITC (Dako, Denmark) diluted in 1:1000 in PBS containing 0.1\% Triton X-100 and 0.1\% DL-lysine for 90 minutes. Thereafter, the segments were washed three times in PBS for 10 minutes and stained for 10 minutes in $0.05 \%$ pontamine sky blue and washed in PBS three times for five minutes. Lastly, the segments were stretched on glass slides and mounted with Vectashield mounting medium. Segments that underwent TH staining were washed three times in Hepes buffer containing $0.1 \%$ Triton $\mathrm{X}-100$ for 10 minutes followed by incubation of $5 \%$ normal swine serum (Jackson immunoresearch, West Grove, PA, USA) in Hepes buffer containing $0.1 \%$ Trion $\mathrm{X}-100$ for 90 minutes and subsequently incubated overnight at room temperature in Rabbit anti-TH (Pel-Freez biologicals Cat\# P40101, RRID:AB-2313713) diluted 1:400 in Hepes buffer containing $0.1 \%$ Triton X-100, $0.1 \%$ DL lysine and $1 \%$ normal swine serum. The next day the segments were washed three times in PBS for 10 minutes and incubated in FITC-conjugated swine anti-Rabbit serum diluted in 1:40 in PBS containing 1\% normal swine serum, $0.1 \%$ Triton $\mathrm{X}-100$ and $0,1 \%$ DL-lysine for 90 minutes. Next, the segments were washed three times in PBS for 10 minutes and stained for 10 minutes in $0.05 \%$ pontamine sky blue and washed in PBS three times for five minutes. Lastly, the segments were stretched on glass slides and mounted with Vectashield mounting medium.

\section{Image processing and analysis}

For quantification of PGP 9.5, VAChT and TH immunoreactivity at the adventitial-medial border in the segments of the basal cerebral arteries (Figure 2) microscope slides were reviewed using an Axiophot fluorescence microscope (Carl Zeiss, Oberkochen, Germany) 
and images were obtained at 20X using a Leica digital camera (DFC 420C, Leica Camera, AG, Germany) and Leica application Suite acquisition software. Images were imported in the public domain software for image analysis, ImageJ, and subjected to an established method of image analysis ${ }^{18}$ While the observer was blinded to ligation status, the diameters of the basal cerebral arteries were measured and in each image, the area percentage (percentage of specific fluorescence in the measuring frame) was scored in three randomly chosen fields, which were subsequently averaged and used for further analysis.

The two consecutively made MRA images were combined into one image for visual inspection (observer blinded to ligation status) where the anterior part of the posterior image was removed in favour of the anterior image. The images were also normalized to compensate for the signal decay as a result of the use of a surface coil. In each individual MRA image the area containing the basilar artery was manually selected. The number of voxels with a signal intensity above a threshold of $20 \%$ of the maximum signal intensity in the selected area was counted as belonging to the basilar artery. Multiplication with the voxel dimensions thus provides apparent artery volumes. Apparent CBF values were obtained by manually selecting the brain in the last images of the non-slice-selective inversion series. The signal intensity of this region of interest (ROI) was summed for each image in each inversion series. The signal difference between the signal intensity of the non-slice-selective inversion series was subtracted from the slice-selective image series. Fitting of the signal difference curve to a modified equation has been previously described by Gunther et al. (2001) and Kober et al. (2008). Time-dependent signal intensities as a result of the BOLD effect during a carbogen challenge were obtained by manually outlining the rat's brain in the images and averaging the signal intensity of all voxels in the brain. This signal was normalized by dividing it with the mean signal intensity obtained during the air/ $/ \mathrm{O}_{2}$ ventilation period at the start. Changes in signal intensity were observed relative to this signal intensity.

\section{Sample size calculation and statistical analysis}

This study had a statistical power of $90 \%$ to identify a difference of 3.0 area-percent nerve density, given a two-sided a value of 0.05 and a standard deviation of $1.7^{10}$. Sample size calculation for detection of the difference between ligated and sham-operated rats, resulted in the need of eight rats in each of the six subgroups (PGP 9.5, VAChT and TH staining in ligated as well as in control rats); a total of 48 rats were required. Continuous variables are presented as median (IQR: interquartile range). Due to the limited sample size, non- parametric tests were used. Nerve densities between groups were compared using the Mann-Whitney $U$ test, whereas the Friedman test was used for comparison of multiple groups with repeated measures. All analyses were performed using commercially available statistical software (SPSS 20.0, SPSS Inc, Chicago, II). A two-sides p-value $<0.05$ was considered significant. 


\section{Results}

\section{Ligation procedure and macroscopical analysis}

All rats survived the whole study period. Two rats developed a subcutaneous hematoma following surgery, which was relieved during the second procedure. In six rats unilateral ptosis was observed postoperatively, which persisted till termination. Based on in vivo MR angiograms uncertainty on the achieved occlusion existed in two rats (MR-group). Post mortem macroscopical evaluation revealed unsuccessful ligation in these and in three other (non-MR group) rats. Subsequently, these five rats were excluded for further analysis, according the protocol. In the sham-operated rats, the CCAs were patent at $\mathrm{t}=0, \mathrm{t}=1$ and $\mathrm{t}=2$.

\section{MRI}

In all ligated rats an increase in tortuosity of the posterior communicating artery (Pcom segment) and first part of the posterior cerebral artery (P1 segment) and the basilar artery was observed at $\mathrm{t}=1$ and $\mathrm{t}=2$, compared to $\mathrm{t}=0$. (see Figure 3 ). In the ligated rats a significantly increased median volume of the basilar artery $(p=0.05)$ and relative signal intensity in the basilar artery $(p=0.04)$ were measured (Table 1a). In the sham operated rats, no tortuosity of the basilar artery was observed at $t=0, t=1$ and $t=2$. Median volumes and relative median signal intensity of the basilar artery remained unchanged (both $p=0.72$ ).

\section{Carbogen challenge}

In all rats an increase in signal intensity was observed at $\mathrm{t}=0, \mathrm{t}=1$ and $\mathrm{t}=2$ for both the switch from air to oxygen and for the switch from oxygen to carbogen, without significant difference between sham-operated and ligated rats.

\section{Perfusion measurements}

No significant differences in apparent CBF between the ligated and sham-operated rats were found (Table 1b). The observed differences fell within the natural range of deviations.

\section{Immunohistochemistry analysis}

As shown in Table 2 the diameters of the first part of the anterior cerebral artery (A1; median $1.00 \mathrm{~mm}$, IQR: $0.88-1.08$, versus median $0.82 \mathrm{~mm}$, IQR: $0.77-0,93, p=0.03$ ), first part of the carotid artery (ICA1; median $1.21 \mathrm{~mm}$, IQR: $1.09-1.27$ versus median $1.06 \mathrm{~mm}$, IQR: 1.00-1.17, $\mathrm{p}=0.03$ ), second part of the carotid artery (ICA2; median $1.15 \mathrm{~mm}$, IQR: $1.02-1.24$ versus median $0.98 \mathrm{~mm}$, IQR: 0.88- 1.12, $\mathrm{p}=0.02$ ), P1 segment (median $0.99 \mathrm{~mm}$, IQR: 0.82- 1.14 versus median $0.83 \mathrm{~mm}, \mathrm{IQR}$ : 0.78-0.89, $\mathrm{p}=0.05$ ) and Pcom segment (median $0.99 \mathrm{~mm}$, IQR: 0.82- 1.14 versus median $0.65 \mathrm{~mm}, \mathrm{IQR}: 0.48-0.69, \mathrm{p}<0.001$ ) in the ligated rats were significantly larger compared to diameters in the sham-operated rats. 
Part II Chapter 8
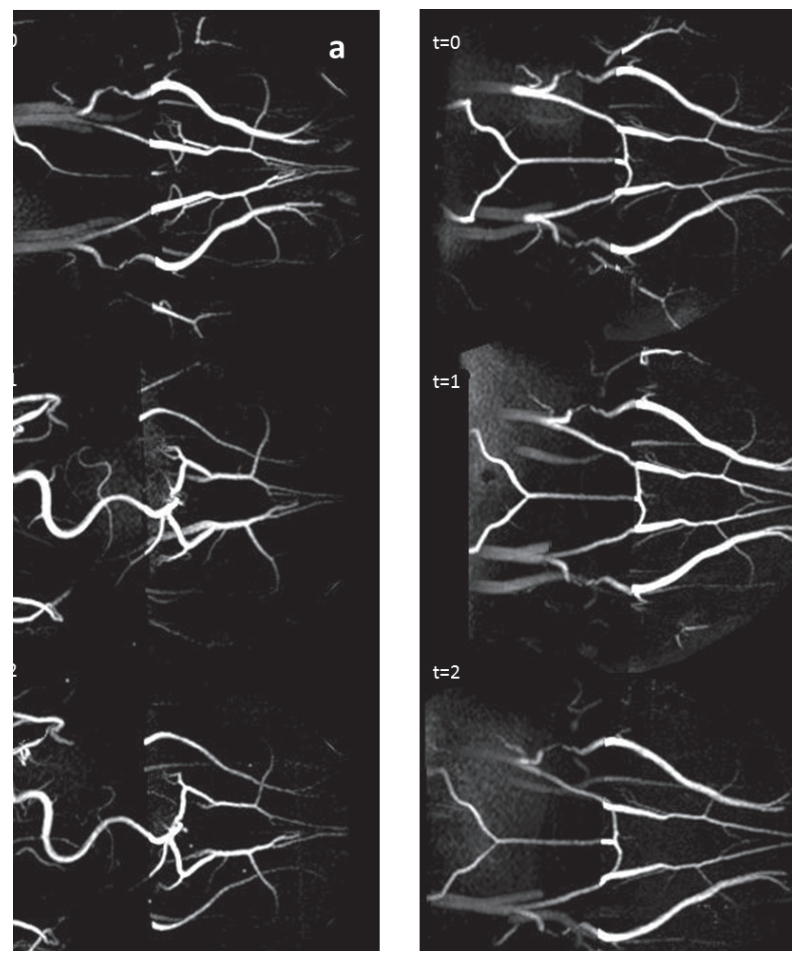

Figure $3 \mathrm{MR}$ images of the three animals of the ligated (a) and sham-operated (b) group at $\mathrm{t}=0$ (preoperative), $\mathrm{t}=1$ (two weeks after bilateral ligation) and $\mathrm{t}=2$ (four weeks after bilateral ligation).

Table 1 Results of MRI volume and perfusion measurements of ligated and sham-operated rats

\begin{tabular}{|c|c|c|c|c|c|}
\hline \multicolumn{2}{|c|}{ Basilar artery volumes $(\mu \mathrm{l})$} & \multirow{2}{*}{$\begin{array}{l}\mathbf{t}=\mathbf{0} \\
16 \\
(14-18)\end{array}$} & \multirow{2}{*}{$\begin{array}{l}\mathbf{t}=1 \\
29 \\
(26-47)\end{array}$} & \multirow{2}{*}{$\begin{array}{l}\mathbf{t}=\mathbf{2} \\
34 \\
(25-42)\end{array}$} & \multirow{2}{*}{$\begin{array}{l}p \text { value } \\
0.05^{\star}\end{array}$} \\
\hline Ligated & $\begin{array}{l}\text { Median } \\
(\mathrm{IQR})\end{array}$ & & & & \\
\hline Sham & $\begin{array}{l}\text { Median } \\
\text { (IQR) }\end{array}$ & $\begin{array}{l}20 \\
(19-21)\end{array}$ & $\begin{array}{l}22 \\
(18-29)\end{array}$ & $\begin{array}{l}21 \\
(18-28)\end{array}$ & 0.72 \\
\hline \multicolumn{6}{|c|}{ Relative signal intensity (\%) } \\
\hline Ligated & $\begin{array}{l}\text { Median } \\
\text { (IQR) }\end{array}$ & $\begin{array}{l}0.0031 \\
(0.0018-0.0045)\end{array}$ & $\begin{array}{l}0.011 \\
(0.0097-0.0122)\end{array}$ & $\begin{array}{l}0.0095 \\
(0.0074-0.0107)\end{array}$ & $0.04^{*}$ \\
\hline Sham & $\begin{array}{l}\text { Median } \\
\text { (IQR) }\end{array}$ & $\begin{array}{l}0.0040 \\
(0.0036-0.0068)\end{array}$ & $\begin{array}{l}0.0048 \\
(0.0016-0.0051)\end{array}$ & $\begin{array}{l}0.0072 \\
(0.0043-0.0079)\end{array}$ & 0.72 \\
\hline \multicolumn{6}{|l|}{ b. } \\
\hline $\begin{array}{l}\text { CBF } \\
(\mathrm{ml} / 100 \mathrm{~g})\end{array}$ & & $t=0$ & $t=1$ & $t=2$ & $p$ value \\
\hline Ligated & $\begin{array}{l}\text { Median\% } \\
\text { (IQR) }\end{array}$ & $\begin{array}{l}303 \\
(283-334)\end{array}$ & $\begin{array}{l}278 \\
(211-287)\end{array}$ & $\begin{array}{l}268 \\
(218-313)\end{array}$ & 0.26 \\
\hline Sham & $\begin{array}{l}\text { Median\% } \\
\text { (IQR) }\end{array}$ & $\begin{array}{l}299 \\
(232-580)\end{array}$ & $\begin{array}{l}312 \\
(236-393)\end{array}$ & $\begin{array}{l}269 \\
(224-317)\end{array}$ & 0.37 \\
\hline
\end{tabular}

Median values and interquartile range (IQR) volumes $(\mu \mathrm{l})$ and relative signal intensity (\%) of the basilar artery (A) and apparent cerebral blood flow values (CBF, B: $\mathrm{ml} / 100 \mathrm{~g} / \mathrm{min}$ ) in the ligated and sham-operated rats at $\mathrm{t}=0$ (preoperative), $\mathrm{t}=1$ (two weeks after bilateral ligation) and $\mathrm{t}=2$ (four weeks after bilateral ligation). Statistically significant differences are indicated by an asterisk $\left(^{*}\right)$. 
Table 2 Diameters of investigated artery segments of ligated and sham-operated rats

\begin{tabular}{|c|c|c|c|c|c|c|c|c|c|}
\hline $\begin{array}{l}\text { Diameter } \\
(\mathrm{mm})\end{array}$ & ICA1 & ICA2 & MCA & A1 & A2 & P1 & P2 & Pcom & Bas \\
\hline \multicolumn{10}{|l|}{ Ligated } \\
\hline $\begin{array}{l}\text { Median } \\
\text { (IQR) }\end{array}$ & $\begin{array}{l}1.21 \\
(1.09- \\
1.27)\end{array}$ & $\begin{array}{l}1.15 \\
(1.02- \\
1.24)\end{array}$ & $\begin{array}{l}0.94 \\
(0.68- \\
1.11)\end{array}$ & $\begin{array}{l}1.00 \\
(0.87- \\
1.08)\end{array}$ & $\begin{array}{l}0.99 \\
(0.73- \\
1.07)\end{array}$ & $\begin{array}{l}0.99 \\
(0.82- \\
1.14)\end{array}$ & $\begin{array}{l}0.74 \\
(0.58- \\
0.89)\end{array}$ & $\begin{array}{l}0.99 \\
(0.82- \\
1.14)\end{array}$ & $\begin{array}{l}0.97 \\
(0.84- \\
1.30)\end{array}$ \\
\hline$N$ & 12 & 13 & 12 & 14 & 13 & 13 & 15 & 12 & 4 \\
\hline \multicolumn{10}{|l|}{ Sham } \\
\hline $\begin{array}{l}\text { Median } \\
(\mathrm{IQR})\end{array}$ & $\begin{array}{l}1.06 \\
(1.00- \\
1.17)\end{array}$ & $\begin{array}{l}0.98 \\
(0.88- \\
1.12)\end{array}$ & $\begin{array}{l}0.75 \\
(0.68- \\
0.82)\end{array}$ & $\begin{array}{l}0.82 \\
(0.77- \\
0.93)\end{array}$ & $\begin{array}{l}0.90 \\
(0.75- \\
1.03)\end{array}$ & $\begin{array}{l}0.83 \\
(0.78 \\
0.89)\end{array}$ & $\begin{array}{l}0.69 \\
(0.60- \\
0.79)\end{array}$ & $\begin{array}{l}0.65 \\
(0.48- \\
0.69)\end{array}$ & $\begin{array}{l}0.95 \\
(0.81- \\
1.04)\end{array}$ \\
\hline $\mathrm{N}$ & 19 & 20 & 21 & 21 & 17 & 18 & 20 & 16 & 4 \\
\hline$p$ value & $0.03^{*}$ & $0.02^{*}$ & 0.08 & $0.03^{\star}$ & 0.49 & $0.05^{\star}$ & 0.40 & $<0.001^{*}$ & 0.56 \\
\hline
\end{tabular}

Median diameters $(\mathrm{mm})$ and interquartile range (IQR) in various segments of the basal cerebral arteries from ligated and sham-operated animals. ICA1: first part of the internal carotid artery, ICA2: second part of the internal carotid artery, MCA: middle cerebral artery, A1: first part of the anterior cerebral artery, A2: second part of the anterior cerebral artery, P1: first part of the posterior cerebral artery, P2: second part of the posterior cerebral artery, Pcom: posterior communicating artery, BAS: basilar artery. Statistically significant values are indicated by an asterisk $\left(^{*}\right)$.

\section{PGP 9.5 immunoreactivity}

In all studied segments PGP 9.5 immunoreactivity was seen in the deep nerve plexus at the adventitial medial border. The PGP 9.5 nerve density was greatest in the anterior segments of the basal cerebral arteries (Table 3a, Figure 4). The ligated animals compared to the shamoperated animals had a significantly higher nerve density, in both the P1 segment, median area 10.1\% (IQR:8.7- 10.5) versus 6.6\% (IQR: 5.5- 7.4), $p=0.003$ and the Pcom segment, median area $6.4 \%$ (IQR: $5.8-6.5$ ) versus $3.2 \%$ (IQR: $2.4-4.3$ ), $p=0.003$ (Figure 5). When rats with ptosis were excluded, the nerve density in the P1 segment and Pcom segment were still significantly different between the ligated and sham-operated rats (both $p=0.006$, data not shown).

\section{VAChT immunoreactivity}

VAChT staining generally resulted in unsatisfactory colour contrast, making it impossible to perform computerized image analysis of VAChT reactivity (Figure 4). Visual impression of the presence of VAChT containing nerves revealed that VAChT was present in the circular oriented terminal plexus at the adventitial medial border in all investigated segments.

\section{TH immunoreactivity}

In all studied segments TH immunoreactivity was observed in the deep nerve plexus at the adventitial medial border (Table 3b, Figure 4). The topographical distribution did not differ between the ligated and sham-operated group. The highest sympathetic nerve density was found in the anterior segment of the circle of Willis. Nerve density in the Pcom segment was significantly higher in the ligated group than the sham-operated group, median $3.7 \%$ (IQR: 2.8-4.8) versus $1.7 \%$ (IQR: 1.3- 2.2, $p=0.02$ ) (Figure 5). When rats with ptosis were excluded, the difference remained significant $(p=0.02$, data not shown). 

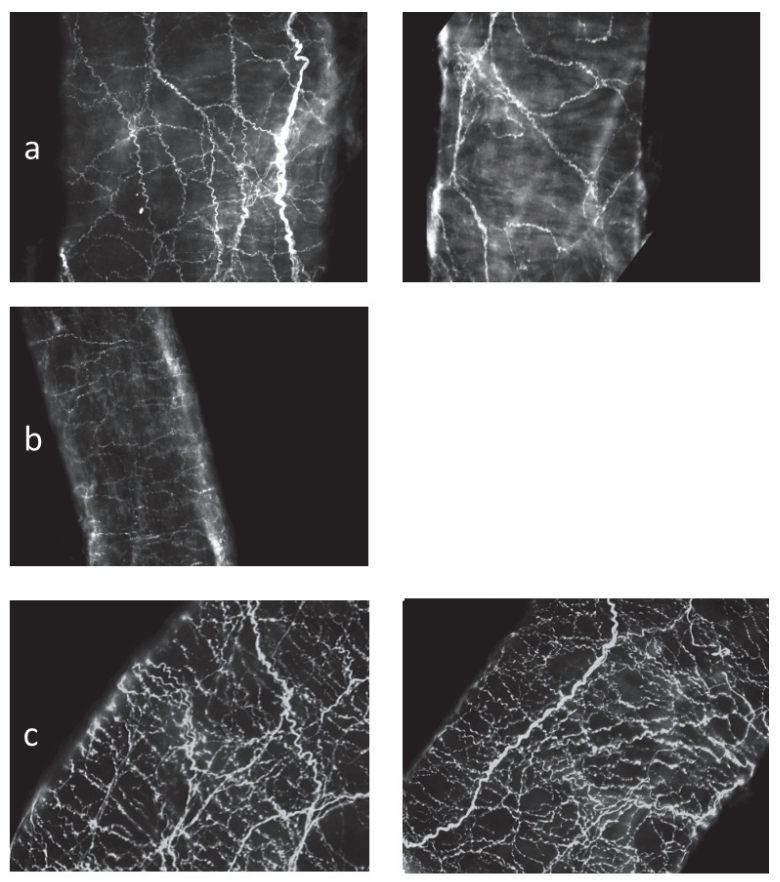

Figure 4 a. Whole mount preparations of perivascular nerves stained for PGP 9.5, Pcom segment of a ligated (left) and a sham-operated rat (right). b. Whole mount preparations of perivascular nerves stained for vesicular acetylcholine transporter, P1 segment of a ligated rat. c. Whole mount preparations of perivascular nerves stained for $\mathrm{TH}, \mathrm{A} 1$ segment of a ligated (left) and sham-operated (right) rat (not significant). For abbreviations see Table 2 legend.
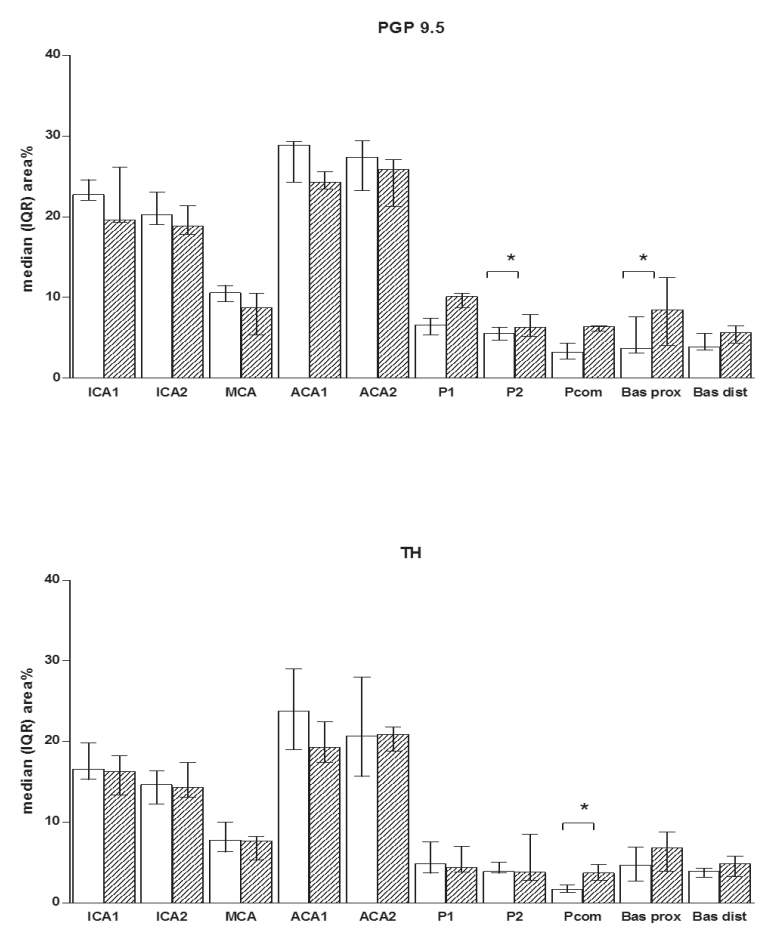

Figure 5 Median values and interquartile range (IQR) area percentage of PGP 9.5-immunoreactive nerve fibers (a) and TH-immunoreactive nerve fibers (b) in various segments of the basal cerebral arteries from shamoperated (white) and ligated animals (striped). For abbreviations see Figure 2 legend. Statistically significant values are indicated by an asterisk (*) 


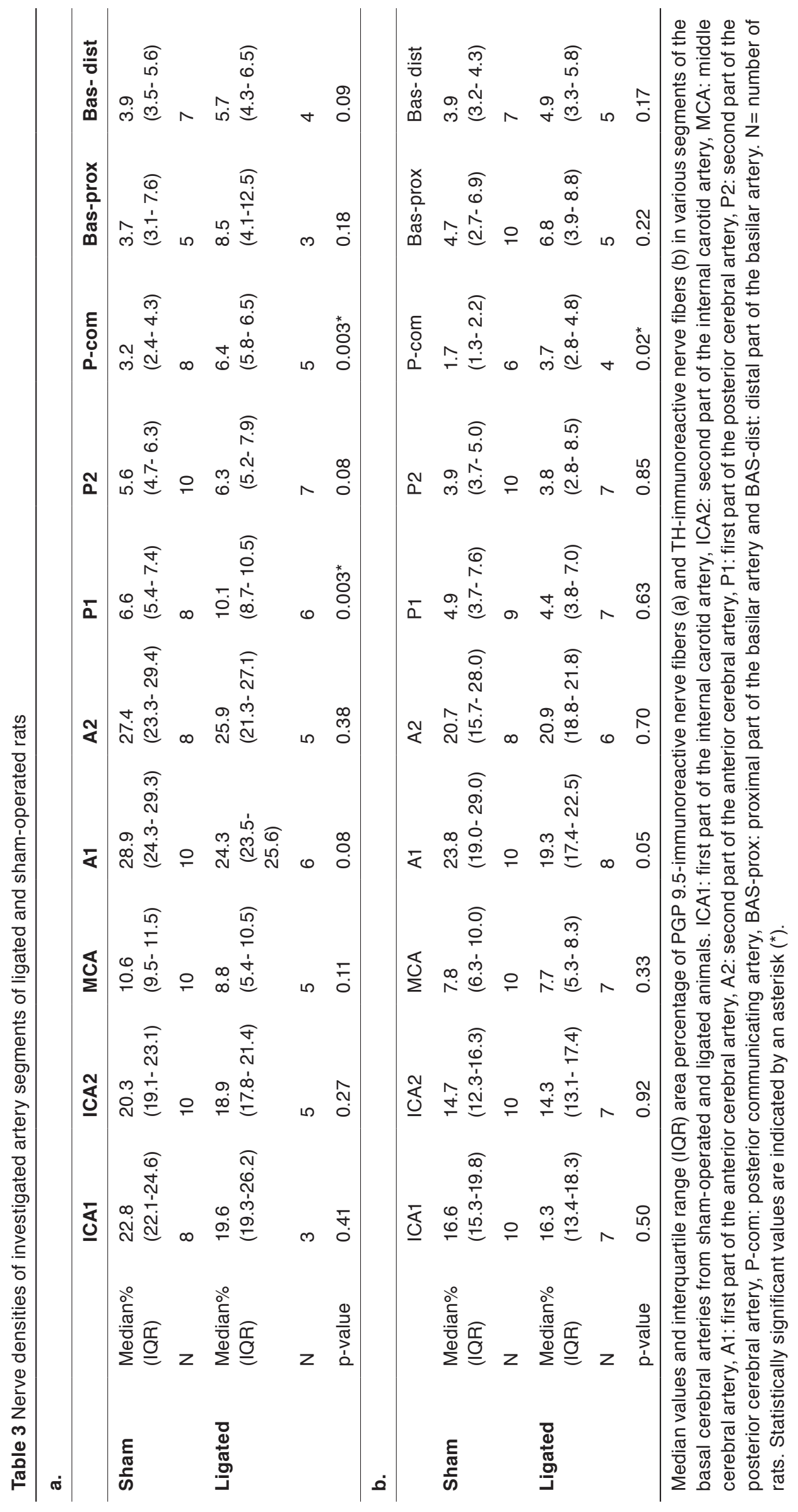




\section{Discussion}

The major findings of this study are the remarkable changes in morphology and nerve density of the posteriorly located basal cerebral arteries that developed after bilateral CCA ligation in rats. The procedure induced a major redistribution of blood supply to the head accompanied by increased flow fluctuations through the vertebral and basilar arteries, resulting in increased basilar artery and Pcom tortuosity, enlargement of several segments and concurrent increase in the nerve supply of the Pcom and P1 segments. However, in the applied animal model no significant changes in cerebral perfusion or cerebrovascular reactivity were measured during MRI follow-up. These findings suggest that the brain's undiseased vascular system has a large adaptation range to compensate the bilateral CCA ligation.

\section{Changes in cerebral arterial morphology and haemodynamics}

Previously, bilateral CCA occlusion in rodents has been performed to study the effects of chronic hypoperfusion on cognitive dysfunction, neurogenerative processes, ischaemic white matter injury and ischaemic eye disease ${ }^{13}$. The fact that bilateral CCA occlusion did not result in significant chronic cerebral hypoperfusion in the current study may be explained by the surgical procedure. In our experience (unpublished data), confirmed by others ${ }^{14}$, contemporaneously bilateral CCA ligation in rats was followed by progressive neurologic deterioration in the first 48 hours postoperatively with a survival rate of $<10-40 \%$. Therefore we used a modified surgical procedure with a one-week interval between the ipsilateral and contralateral ligation to avoid a too abrupt reduction of CBF, resulting in a $100 \%$ survival rate $^{19}$. It has been shown previously that gradual occlusion of a vessel results in a less deleterious effect on the tissue elements of the distal territory in comparison to an abrupt occlusion ${ }^{20}$, while changes in cognitive impairment are comparable to the conventional model. Possibly, CBF was reduced immediately after ligation of the second CCA, but normalized when measured by MRI two weeks after surgery which is in agreement with a similar rabbit model suggesting adaption to normal perfusion within 5 days ${ }^{21}$. Chronic CBF recovery after bilateral CCA ligation in rats has been observed previously, however CBF remained slightly but significantly decreased up to at least four weeks after bilateral CCA ligation as compared to controls ${ }^{22}$. An increase of vascular diameter was observed in all intracircular segments of the basal cerebral arteries and in ICA1. This was expected for the vessels that connect the basilar and the internal carotid arteries, Pcom and P1, of which the PCom, connecting the posterior and anterior parts of the circle, showed the highest increase (52\%). Apparently, flow redistribution following the bilateral carotid ligation all anastomosing segments of the circle of Willis have to adapt to ensure sufficient cerebral perfusion. Dilatation of the afferent ICA1 (distal to the ligation), secondary to retrograde supply to external carotid branches, may reflect its inability to release its contents. Interestingly, the basilar artery did not show an increase in diameter during microscopical analysis but showed elongation and tortuosity during in vivo MR only. The diameters of all efferent arteries, A2, MCA and P2, did not change in agreement with a normal cerebral perfusion. 
As reported in a previous study, we observed that the cerebrovascular reactivity was not affected two weeks following bilateral CCA ligation, This suggests that vasodilatation is not the primary mechanism for CBF maintenance or recovery ${ }^{23}$.

\section{Changes in perivascular nerve density}

The current observation that nerve density in the posteriorly located basal cerebral arteries increases with a rise in blood flow, is in line with previous findings that the regional nerve density correlates with local blood flow ${ }^{23}$ and has the capacity to adapt to altering functional demands ${ }^{9}$. Moreover, perivascular sympathetic nerves are capable of effectuating alterations in cerebral perfusion ${ }^{24,25}$ and elimination of sympathetic innervation has been shown to protect from cerebral hypoperfusion under ischaemic conditions ${ }^{8}$. It seems that changes in flow and pressure trigger the cerebrovascular innervation to adapt until a new optimal innervation pattern is reached. We hypothesize that neurotrophic factors are released in response to local changes in flow and pressure, resulting in an increase in sympathetic nerve density in order to keep CBF within normal range. Our findings support the hypothesis that the sympathetic nerves play a functional role in regulation of $\mathrm{CBF}$ and are therefore part of the autoregulation mechanism. Considering the observed patterns of altered diameters and nerve densities we suggest that the haemodynamic changes which occurred had to reach a threshold in order to trigger these morphological changes.

\section{Limitations}

Because of restrictions in the number of subgroups and number of animals to be used in this study, we chose to terminate all rats after four weeks, which was considered to be the most likely period to detect permanent changes based on a prior study ${ }^{10}$. It is unclear whether the changes in nerve density as observed in the current study are temporary or permanent. It may be interesting to study whether these changes are reversible and disappear after restoration of blood flow in the carotid artery. From the clinical point of view, it would be of importance to understand autoregulatory mechanisms after operating the contralateral carotid artery in case of ipsilateral carotid occlusion for the need for intraoperative shunting, as well as the risk for postoperative hyperperfusion. Nonetheless, we are presently unaware on the clinical significance of the found differences in nerve densities. We ligated the CCA and not solely the ICA. Therefore the entire cranial circulation was affected instead of only the intracranial part. The reason for this approach was the close topographical relationship of the ICA and the superior cervical ganglion and as a consequence a potential disruption of the sympathetic innervation of the cerebral arteries when performing a ligation of the ICA.

The used MR methods can be discussed. The TOF method is dependent on the arterial flow and flow velocity. The initial flow through the rat's basilar artery may have been too low for robust visualization using MRA with the chosen settings. After bilateral CCA occlusion the flow presumably increases, which improves the reliability of detection of the basilar artery. Consequently, the measurements of the volume of the vessel in MRA should be interpreted with caution and data were used to further support our findings of increased diameters in the 
ligated group. As these changes did not occur in the sham-operated group we believe that these changes are most likely due to the ligation procedure.

In six rats ptosis was observed, however as no significant difference between rats with and without ptosis was found, we do not believe the ptosis was a symptom of a complete interruption of the sympathetic nervous system and we did not exclude these rats from our analysis. VAChT staining resulted in poor color contrast, making it impossible to perform computerized image analysis. This may be due to the localization of the antigen. VAChT is involved in transporting acetylcholine into synaptic vesicles and therefore it is mainly present in neuron cell bodies, where it is synthesized, and in nerve terminals. In the autonomic nervous system transmitters are released from varicosities which are distributed over the terminal nerve plexuses. As a result, the staining shows many small spots which do not always allow for computerized image analysis.

\section{Translation to humans}

Animal studies are required to gain more insight on the influence of redistribution of blood flow on cerebrovascular innervation. Moreover, in experimental animal studies influence of several other factors such as degenerative processes, ageing, silent cerebral embolism, and diabetes mellitus can be excluded ${ }^{25}$. A different topographical distribution of the nerve density in the vascular system between humans and rats does exist. In humans the posterior segment of the cerebral circulation has been described as most densely innervated, whereas in rats the highest nerve density was found in the anterior cerebral artery ${ }^{9,26}$. As this contrast might be due to a high metabolic need of the target vascular territory, (i.e. the visual cortex in humans and rhinencephalic structures in rats), we do not believe this difference influenced our results. We acknowledge that in humans, as opposed to healthy rats, carotid artery disease is an indicator of generalized atherosclerosis and that intracranial carotid artery disease often coexists ${ }^{27}$. In addition, progressive generalized atherosclerosis may limit compensatory mechanisms in man, ultimately resulting in watershed infarction.

\section{Conclusion}

Our findings in the rat model provide support for the hypothesis that bilateral CCA occlusion results in alterations in cerebral haemodynamics and nerve density and underline close morphological and functional relationships of working mechanisms in the basal cerebral arteries.

\section{Acknowledgements}

We thank mr. J.W.de Groot for assistance with immunohistochemistry and dr. T.A.P. Roeling for technical help with image analysis. 


\section{References}

1. Klijn CJM, Kappelle LJ, Tulleken CAF, van Gijn JM. Symptomatic Carotid Artery Occlusion: A Reappraisal of Hemodynamic Factors. Stroke. 1997;28(10):2084-2093.

2. Powers WJ. Cerebral hemodynamics in ischemic cerebrovascular disease. Ann Neurol. 1991;29(3):231-40.

3. Silesen H, Schroeder T. Haemodynamic evaluation of carotid artery disease. Clin Phys Physiol Meas. 1989;10 Suppl A:15-22.

4. Ursino M. Regulation of the circulation of the brain. In: Bevan RD, Bevan JA The human brain circulation: functional changes in disease Totowa, New Jersey: Humana Press. 1994. p. 291-318.

5. Cipolla MJ. Control of cerebral blood flow. In: The cerebral circulation. San Rafael: Morgan \& Claypool Life Sciences; 2009.

6. Silva D, Belli A. Critical care management of neurosurgical patients. In: Ellebogen RG, Sekhar LN, Kitchen N, editors. Principles of Neurological Surgery. 4th ed. philadelphia: Elsevier; 2018. p. 392.

7. Roloff E v. L, Tomiak-Baquero AM, Kasparov S, Paton JFR. Parasympathetic innervation of vertebrobasilar arteries: is this a potential clinical target? J Physiol. 2016;594(22):6463-85.

8. Lee RH, Couto e Silva A, Lerner FM, Wilkins CS, Valido SE, Klein DD, et al. Interruption of perivascular sympathetic nerves of cerebral arteries offers neuroprotection against ischemia. Am J Physiol - Hear Circ Physiol. 2017;312(1):H182-8.

9. Bleys RLAW, Cowen T. Innervation of Cerebral Blood Vessels : Morphology, Plasticity, Age-Related, and Alzheimer 's Disease-Related Neurodegeneration. Microsc Res Tech. 2001;53:106-18.

10. Van Denderen JCM, Van Wieringen GW, Hillen B, Bleys RLAW. Zinc sulphate-induced anosmia decreases the nerve fibre density in the anterior cerebral artery of the rat. Auton Neurosci Basic Clin. 2001;94(1-2):102-8.

11. Bleys RL, Cowen T, Groen GJ, Hillen B. Perivascular Nerves of the Human Basal Cerebral Arteries II . Changes in Aging and Alzheimer â€ ${ }^{\mathrm{TM}}$ s Disease. J CerebBlood Flow Metab. 1996;16(5):1048-57.

12. Martin AJ, Friston KJ, Colebatch JG, Frackowiak RSJ. Decreases in Regional Cerebral Blood Flow with Normal Aging. J Cereb Blood Flow Metab. 1991;11(4):684-9.

13. Farkas E, Luiten PGM, Bari F. Permanent, bilateral common carotid artery occlusion in the rat: A model for chronic cerebral hypoperfusion-related neurodegenerative diseases. Brain Res Rev. 2007;54(1):162-80.

14. Cechetti F, Worm P V., Pereira LO, Siqueira IR, Netto CA. The modified 2VO ischemia protocol causes cognitive impairment similar to that induced by the standard method, but with a better survival rate. Brazilian J Med Biol Res. 2010;43(12):1178-83.

15. Gunther M, Bock M, Schad LR. Arterial spin labeling in combination with a look-locker sampling strategy: Inflow turbo-sampling EPI-FAIR (ITS-FAIR). Magn Reson Med. 2001;46(5):974-84.

16. Kober F, Duhamel G, Cozzone PJ. Experimental comparison of four FAIR arterial spin labeling techniques for quantification of mouse cerebral blood flow at 4.7 T. NMR Biomed. 2008;21:781-92.

17. Cowen T, Haven a J, Burnstock G. Pontamine sky blue: a counterstain for background autofluorescence in fluorescence and immunofluorescence histochemistry. Histochemistry. 1985;82(3):205-8.

18. Cowen T, Thrasivoulou C. Cerebrovascular nerves in old rats show reduced accumulation of 5-hydroxytryptamine and loss of nerve fibres. Brain Res. 1990;513(2):237-43.

19. Tsuchiya M, Sako K, Yura S, Yonemasu Y. Cerebral blood flow and histopathological changes following permanent bilateral carotid artery ligation in Wistar rats. Exp Brain Res. 1992;89(1):87-92.

20. Kaliszewski C, Fernandez LA, Wicke JD. Differences in mortality rate between abrupt and progressive carotid ligation in the gerbil: Role of endogenous angiotensin II. J Cereb Blood Flow Metab. 1988;8(2):149-54.

21. Tutino VM, Mandelbaum M, Choi H, Pope LC, Siddiqui A, Kolega J, et al. Aneurysmal remodeling in the circle of Willis after carotid occlusion in an experimental model. J Cereb Blood Flow Metab. 2014;34(3):415-24.

22. Otori T, Katsumata T, Muramatsu H, Kashiwagi F, Katayama Y, Terashi A. Long-term measurement of cerebral blood flow and metabolism in a rat chronic hypoperfusion model. Clin Exp Pharmacol Physiol. 2003;30(4):26672.

23. Choy M, Ganesan V, Thomas DL, Thornton JS, Proctor E, King MD, et al. The chronic vascular and haemodynamic response after permanent bilateral common carotid occlusion in newborn and adult rats. $J$ Cereb Blood Flow Metab. 2006;26(8):1066-75.

24. Edvinsson L, Hamel E. Perivascular Nerves in Brain Vessels. In: Edvinsson L, Krause DN, editors Cerebral Blood Flow and Metabolism Lippincott Williams \& Wilkins. 2002.

25. Sercombe R, Aubineau P, Edvinsson L, Mamo H, Pinard E, Seylaz J. Neurogenic influence on local cerebral blood flow. Neurology. 1975;25(October):954-63.

26. Bleys RL, Cowen T, Groen GJ, Hillen B. Perivascular Nerves of the Human Basal Cerebral Arteries: I. Topographical Distribution. J CerebBlood Flow Metab. 1996;16(0271-678X (Print)):1048-57.

27. Kappelle LJ, Eliasziw M, Fox AJ, Sharpe BL, Barnett HJ. Importance of intracranial atherosclerotic disease in patients with symptomatic stenosis of the internal carotid artery. The North American Symptomatic Carotid Endarterectomy Trail. Stroke. 1999;30(2):282-6. 


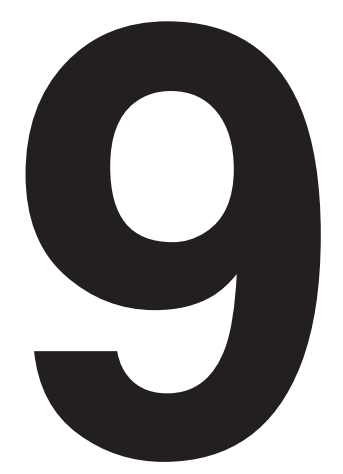

The effect of carotid revascularisation on cerebral arterial function as measured by 7-Tesla MRI

ML Rots, T Arts, J Hendrikse, GJ de Borst, J.J.M. Zwanenburg In preparation 


\section{Abstract}

OBJECTIVE Revascularisation of the carotid artery affects blood flow through the ipsilateral carotid artery. Yet, not much is known about how this influences the function of perforating cerebral arteries. Advances in ultrahigh-field MRI have led to new methods to measure the structure and function of cerebral small vessels (perforators). In this feasibility study we explore the effect of a carotid endarterectomy (CEA) on cerebral arterial function as measured by 7 Tesla MRI.

METHODS Symptomatic carotid artery disease patients underwent 2D Qflow acquisitions aimed at the M1 segment of the middle cerebral artery (MCA) and small perforators in the centrum semi-ovale (CSO) and the basal ganglia (BG) on 7 Tesla MRI. Scans were performed one day prior to and one day after CEA. Cerebral small perforators were detected in two masks; in the CSO and BG. Mean velocity $\left(\mathrm{V}_{\text {mean }}\right)$, pulsatility index $(\mathrm{PI})$ and number of perforators were determined for each of the scans and compared between ipsi- and contralateral as well as between pre- and post-operative scan.

RESULTS Six patients were able to complete pre- and post-operative MRI (four men; mean age $72.5( \pm 5.7))$. CSO, BG and M1 scans were successful in all patients. $V_{\text {mean }}$ of the ipsilateral M1 increased in 5/6 patient (unchanged in 1/6). Ipsilateral M1 PI decreased in 1/6 and increased in 5/6 patients. Ipsilateral $\mathrm{V}_{\text {mean }}$ of CSO perforators increased in $1 / 6$ patients and was unchanged in the remaining five patients while number of detected perforators $/ \mathrm{cm}^{2}$ increased in 4/6 and decreased in 2/6 patients. PI of CSO perforators increased in 2/6 and decreased in $4 / 6$ patients. Ipsilateral $V_{\text {mean }}$ of $B G$ perforators increased in $2 / 6$, decreased in $2 / 6$ and was unchanged 2/6 patients, numbers of detected perforators increased in 3/6 and decreased in $3 / 6$ patients. PI of BG perforators increased in $3 / 6$, decreased in $2 / 6$ and was unchanged in $1 / 6$ patients.

DISCUSSION The relation between large and perforating artery velocity and pulsatility is complex and ambiguous. CEA generally resulted in ipsilateral increase in $\mathrm{V}_{\text {mean }}$ of $\mathrm{M} 1$, without a directly related increase in $\mathrm{V}_{\text {mean }}$ of $\mathrm{CSO}$ and $\mathrm{BG}$ perforators. Future studies should confirm whether these novel imaging techniques can be further deployed for patient selection. 


\section{Introduction}

$10-15 \%$ of all strokes are caused by atherosclerotic stenosis of the internal carotid artery (ICA). Carotid endarterectomy (CEA) and carotid artery stenting (CAS) have been proven to reduce the long-term risk of stroke in patients with symptomatic carotid artery stenosis ${ }^{1}$ with CEA being the first choice of treatment with lower risk of peri-operative cerebrovascular events and silent brain ischemia ${ }^{2,3}$ Revascularization procedures may reduce stroke risk by removal of the atheromatous plaque as a resource of thrombo-embolisms. Nevertheless, optimal treatment strategy, either CEA, CAS or best medical treatment (BMT), in asymptomatic or low-risk symptomatic patients is still under debate ${ }^{4,5}$. Notably, carotid disease patients are also at risk for compromised cerebral haemodynamics, possibly resulting in cognitive decline and cerebral ischemia due to chronic cerebral hypoperfusion ${ }^{6,7}$. Moreover, carotid artery stiffness might result in high pulsatility of small perforating cerebral arteries ${ }^{8}$, which is associated with cerebral small vessel disease ${ }^{9,10}$. In addition to lowering the risk of thrombo-embolic events, carotid revascularization may improve cerebral blood flow and as such haemodynamic aspects may contribute to the discussion on optimal treatment strategy in these patients.

Additionally, cerebral hyperperfusion syndrome (CHS) is a severe complication that develops after carotid revascularization, caused by the sudden upsurge of blood flow through the ipsilateral carotid artery. Screening for patients at risk for $\mathrm{CHS}$ is usually done by measuring increase of flow-velocity in the middle cerebral artery (MCA) with transcranial Doppler (TCD), which has been proven to be a good method for identification of those not at risk for $\mathrm{CHS}^{11}$. However, only a small portion of patients with $\geq 100 \%$ increase in flow velocity after intervention will develop CHS and thus overtreatment (continuous invasive BP monitoring on high care units), prolonged hospitalisation and the associated costs are common practice ${ }^{12}$. Better understanding and prediction of the haemodynamic changes that take place as a result of a carotid revascularization could help in more accurate identification of patients that will develop CHS.

Advances in ultrahigh-field MRI have led to new methods to measure the structure and function of cerebral perforators. Earlier literature has demonstrated the ability to detect small perforating arteries in de the centre semi-ovale (CSO) and basal ganglia (BG) by using a 2D phase contrast angiography with 7 Tesla (7T) MRI and measure blood flow velocity and pulsatility ${ }^{13,14}$. Assessment of these parameters prior to and after a revascularization procedure can demonstrate the direct effect of such a procedure on the perfusion of these perforators. In this feasibility study we aimed to explore changes in the perfusion of the perforating arteries of the brain. 


\section{Methods}

\section{Participants}

Ethical approval for this study was provided by the Medical Research Ethics Committee of University Medical Center Utrecht, Utrecht, the Netherlands on June 12, 2018 (18-315), and all research was conducted according to the principles of the Declaration of Helsinki (59 ${ }^{\text {th }}$ amendment, Seoul 2008) and in accordance with the Dutch Medical Research Involving Human Subjects Act (WMO). Patients included in the study were symptomatic (stroke, hemispheric transient ischaemic attacks (TIA) or monocular blindness $<6$ months prior to hospital presentation) with a high-grade carotid artery stenosis ( $>50 \%$ according to NASCET ${ }^{15}$ ). Indication for CEA was determined by an expert multidisciplinary panel. Exclusion criteria were contraindications for MRI (e.g. metal implants, claustrophobia).

\section{MR image acquisition}

The study protocol consisted of performing a 3 Tesla and a 7 Tesla MRI scan on the day before and the day after surgery in each patient. For the present study only 7T MRI data are analysed and from this point on only the 7T scan protocol and results will be discussed. All 7T acquisitions were performed on a Philips Achieva MRI system (Philips Healthcare, Best, the Netherlands) with a 32-channel receive coil with volume transmit/receive coil for transmission (Nova Medical, Wilmington, MA). The MRI protocol consisted of a $T_{1}$-weighted 3D turbo spin-echo acquisition, a 3D phase contrast angiography (PCA) sequence of the CoW, a 2D phase contrast quantitative flow (2D Qflow) sequence of the white matter in the CSO, the BG and M1 segment of middle cerebral artery (MCA) on the ipsi- and contralateral side. The full protocol for 2D Qflow image acquisition has been published previously ${ }^{13,14}$. In short, the CSO and $B G$ acquisition was retrospectively gated with a $0.3 \mathrm{~mm}$ in-plane resolution and a temporal resolution of $114 \mathrm{~ms}$. $V_{\text {enc }}$ for CSO and BG was 4 and $20 \mathrm{~cm} / \mathrm{s}$ respectively, with a 3:30 min:s acquisition at $80 \mathrm{bpm}$ heartrate. 2D Qflow measurements of the M1 segments were acquired in a similar manner but with $0.5 \mathrm{~mm}$ in-plane resolution and $\mathrm{V}_{\mathrm{enc}}=120 \mathrm{~cm} / \mathrm{s}$.

\section{Image processing}

MR system software (Philips Healthcare, Best, the Netherlands) was used to analyse MCA (M1 segment) velocity, flux and pulsatility. Single-click automatic segmentation was performed for both ipsi- and contralateral M1. When the M1 showed a phase wrap-around artefact due to large velocity-induced phase shift, this was corrected with in-house developed software using the GoldsteinUnwrap2D function (MATLAB R2019a, The MathWorks, Inc) based on the Goldstein method ${ }^{16}$.

The full protocol for detection of small perforators has been published elsewhere ${ }^{13,14}$. In short, cerebral small perforators were detected in two masks; in the CSO and BG. For CSO WM segmentation a 3D $\mathrm{T}_{1}$-weighted MR image was used (SPM12, Wellcome Trust Centre for Neuroimaging). CSO white matter within 80 pixels from the brain surface was excluded to prevent partial volume effects from sulci touching the image plane. The BG were delineated manually based on the $\mathrm{T}_{1}$ image. For the $\mathrm{CSO}$, perforators located in ghosting artefact regions 
were automatically excluded ${ }^{17}$, for the BG perforators non-perpendicular to the PCA imaging plane were excluded. Finally, for the CSO and BG, perforators located within six voxels (1 voxel equals $0.3 \mathrm{~mm}$ ) from each other were excluded since they are more likely to be located on non-perpendicular perforators. Mean flow velocity over the cardiac cycle $\left(\mathrm{V}_{\text {mean }}\right)$ and pulsatility index $(\mathrm{PI})$ in each of the measured perforators (M1, CSO/BG perforators) were compared between the ipsi- and contralateral side and between prior to and after CEA. The PI was calculated from the average time curve as $\left(\mathrm{V}_{\max }-\mathrm{V}_{\text {min }}\right) / \mathrm{V}_{\text {mean }}$. For perforators the included number per $\mathrm{cm}^{2} \mathrm{CSO}$ or BG mask were calculated, for the M1 the mean lumen-area was determined. The differences were explored and described per patient; no comparative statistics were performed due to the limited number of included patients. Visual inspection of the $3 \mathrm{D}$ imaging of the anterior part of the CoW was performed to assess the completeness and flow direction within the CoW before and after CEA.

\section{Secondary outcome measures.}

Clinical events (periprocedural stroke, cerebral hyperperfusion or $\mathrm{CHS}$ ) were listed. Blood pressure values at moment of pre- and postoperative MRI were registered. Flow velocity and PI of the MCA as determined by Transcranial Doppler (TCD) was registered whenever acoustic temporal bone window was adequate.

\section{Results}

From December 2018 until august 2019 seven patients were included of whom six were able to complete both pre- and post-operative scan. Of those six patients four were men (67\%); mean age was $72.5( \pm 5.7)$ year. Table 1 shows the individual patient characteristics. None of the patients had a contralateral occlusion, three out of six patients had a contralateral stenosis of $<50 \%$. CSO, BG and M1 scans were successful in all patients. Figure 1 demonstrates an example 2D Qflow scan of the CSO and BG with the used masks and identified perforators.

\section{M1}

Table 2 shows the results of the ipsi- and contralateral M1 segment of the post-operative compared to pre-operative scan. Median ipsilateral pre-operative $V_{\text {mean }}$ was $32 \mathrm{~cm} / \mathrm{s}$ (range $23-56 \mathrm{~cm} / \mathrm{s}$ ) versus $31 \mathrm{~cm} / \mathrm{s}$ (range $23-54 \mathrm{~cm} / \mathrm{s}$ ) on the contralateral side. $V_{\text {mean }}$ of the ipsilateral $M 1$ increased in the majority of patients with the percentage of increase varying between $-1.4 \%$ and $+165 \%$ (figure 2, panel $\mathrm{A}$ ). Contralateral $\mathrm{V}_{\text {mean }}$ changed between $-2 \%$ and $+63 \%$. Median pre-operative ipsilateral PI was 0.66 (range $0.51-2.42$ ) versus 0.96 on the contralateral side (range $0.85-1.07$ ). Ipsilateral change in PI of the M1 varied between $-57 \%$ and $+101 \%$ (figure 2 , panel B) and contralateral PI change varied between $-15 \%$ and $+33 \%$ when post-operative scan was compared to pre-operative scan. Median baseline ipsilateral M1 lumen-area was $0.86 \mathrm{~cm}^{2}$ (range $0.47-1.04 \mathrm{~cm}^{2}$ ) and $0.84 \mathrm{~cm}^{2}$ (range $0.46-1.1 \mathrm{~cm}^{2}$ ) on the contralateral side. The change in mean M1 lumen-area varied between $-65 \%$ and $+11 \%$ (figure 2, panel C) on the ipsilateral side and $-1-\%$ and $+28 \%$ on the contralateral side. Median ipsilateral flux on 
the pre-operative scan was $1.68 \mathrm{ml} / \mathrm{s}$ (range $1.26-1.94 \mathrm{ml} / \mathrm{s}$ ) versus $1.58 \mathrm{ml} / \mathrm{s}$ (range 1.07 2.05 ) on the contralateral side. The change of flux on the ipsilateral side varied between $-8 \%$ and $+51 \%$ and between $-4 \%$ and $+115 \%$ on the contralateral side.
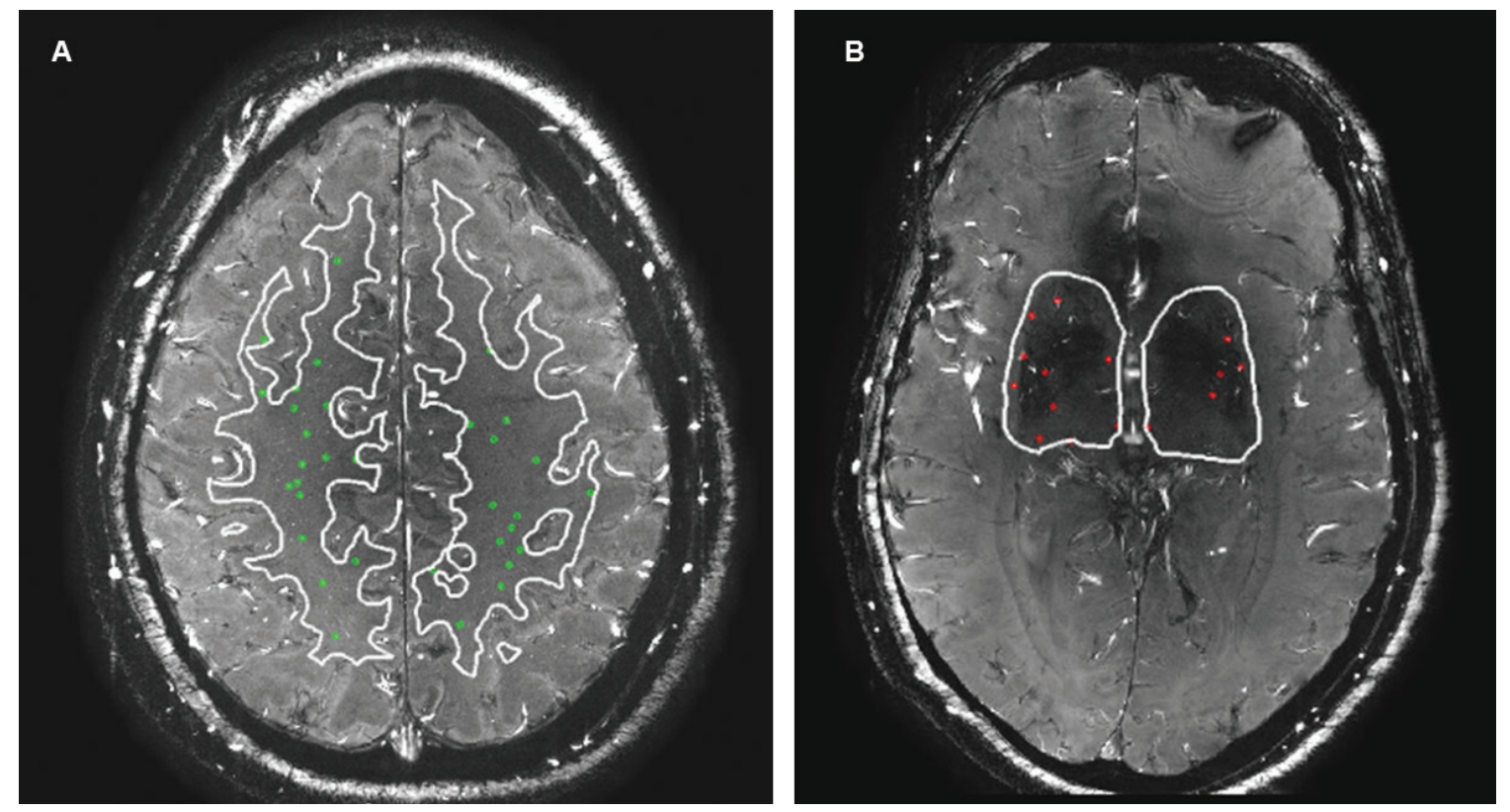

Figure 1 Example 2D QFlow scan of the CSO (panel A) and BG (panel B).

The white lines represent the used mask and green and red and green spheres indicate respectively the detected $\mathrm{CSO}$ and BG perforators within the mask.

Table 1 Baseline characteristics of included patients

\begin{tabular}{llllll}
\hline ID & Age & Gender & Hypertension & Diabetes & Smoking history \\
\hline 1 & 73 & Male & Yes & Yes & No \\
2 & 71 & Male & No & Yes & No \\
3 & 83 & Female & Yes & No & No \\
4 & 67 & Male & Yes & No & Yes \\
5 & 73 & Male & Yes & Yes & No \\
6 & 68 & Female & Yes & No & Yes \\
\hline ID & Hypercholesterolemia & Baseline BP & Stenosis grade & Contralateral & Stenosis \\
& & & & stenosis & Side \\
\hline 1 & No & $125 / 55$ & $70 \%$ & No & Right \\
2 & No & $185 / 90$ & $70 \%$ & $<50 \%$ & Right \\
3 & Yes & $185 / 100$ & $70 \%$ & $<50 \%$ & Left \\
4 & Yes & $165 / 100$ & $70 \%$ & $<50 \%$ & Left \\
5 & Yes & $160 / 85$ & $90 \%$ & No & Right \\
6 & Yes & $135 / 80$ & $90 \%$ & No & Right \\
\hline
\end{tabular}

$\mathrm{V}_{\text {MEAN }}=$ Mean velocity; $\mathrm{PI}=$ Pulsatility Index 


\section{CSO}

A median of 27 (range 8-85) CSO perforators were identified in each of the 12 scans with a median of 14 ipsilateral and 13 contralateral perforators. Table 3 shows the tested parameters of pre-operative scan compared to post-operative scan for both ipsi- and contralateral perforators. Median baseline pre-operative $V_{\text {mean }}$ was $0.75 \mathrm{~cm} / \mathrm{s}$ (range $0.66-1.02 \mathrm{~cm} / \mathrm{s}$ ) on the ipsilateral side versus $0.73 \mathrm{~cm} / \mathrm{s}$ (range $0.60-0.99 \mathrm{~cm} / \mathrm{s}$ ) on the contralateral side. A notable increase in $\mathrm{V}_{\text {mean }}$ in the ipsilateral CSO perforators was demonstrated in one of the six patients (patient 3 , increase of $153 \%$ ), while change of $V_{\text {mean }}$ in other patients varied between $-15 \%$ and $+2 \%$ (figure 3, panel A). Contralateral change of $\mathrm{V}_{\text {mean }}$ in the CSO varied between $-14 \%$ and $+34 \%$. Median baseline ipsilateral PI was 0.64 (range $0.42-0.82$ ) with a contralateral baseline $\mathrm{PI}$ of 0.57 (range $0.45-0.67$ ). Change in $\mathrm{PI}$ in the CSO varied between $-55 \%$ and $+109 \%$ on the ipsilateral side (figure 3, panel B) and $-42 \%$ and $+125 \%$ on the contralateral side. The median number of perforators included per $\mathrm{cm}^{2} \mathrm{CSO}$-mask on pre-operative scan was 0.47 (range $0.10-0.49$ ) and 0.33 (range $0.29-0.76$ ) on the contralateral side. The change in detected perforators varied widely between patients on the ipsilateral side (between $-40 \%$ and $+216 \%$, figure 3 panel C) as well as the contralateral side (between $-55 \%$ and $+487 \%$ ). 

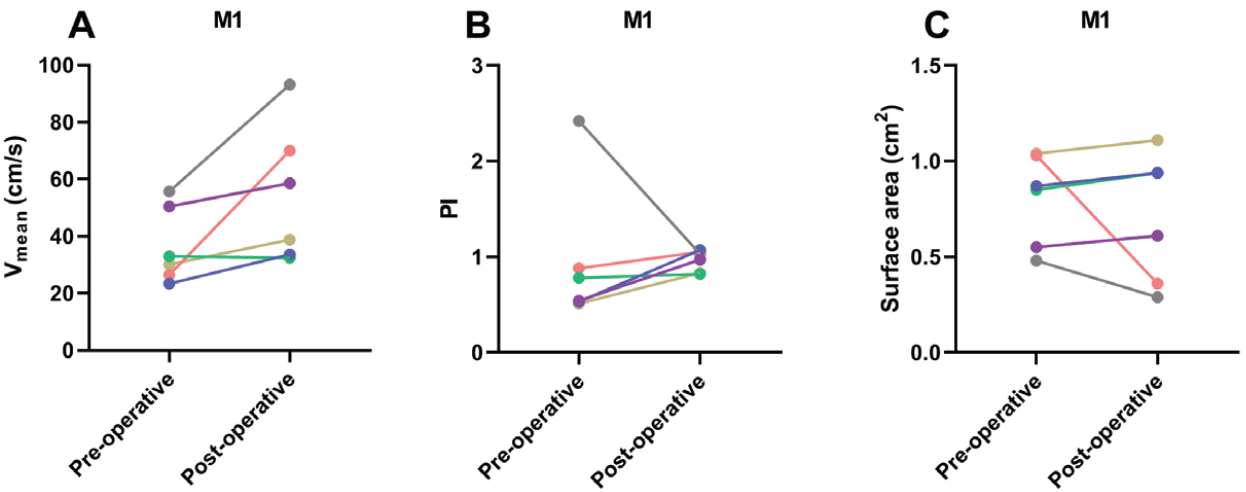

Figure 2 Results of the ipsilateral M1 For each of the patients the post-operative values are plotted against pre-operative values. Panels A, B and C represent respectively ipsilateral Vmean pulsatility index (PI) and the mean lumen area (cm2) for the ipsilateral M1. Vmean increases in the majority of patients with the percentage of increase varying between $-1.4 \%$ and +165 . Change in $\mathrm{PI}$ varied between $-58 \%$ and $+17 \%$. The lumen area notably decreased in one patients (orange line $=$ patient 3 , decrease of $-65 \%$ ), change in lumen area in other patients varied between $-39 \%$ and $+11 \%$.
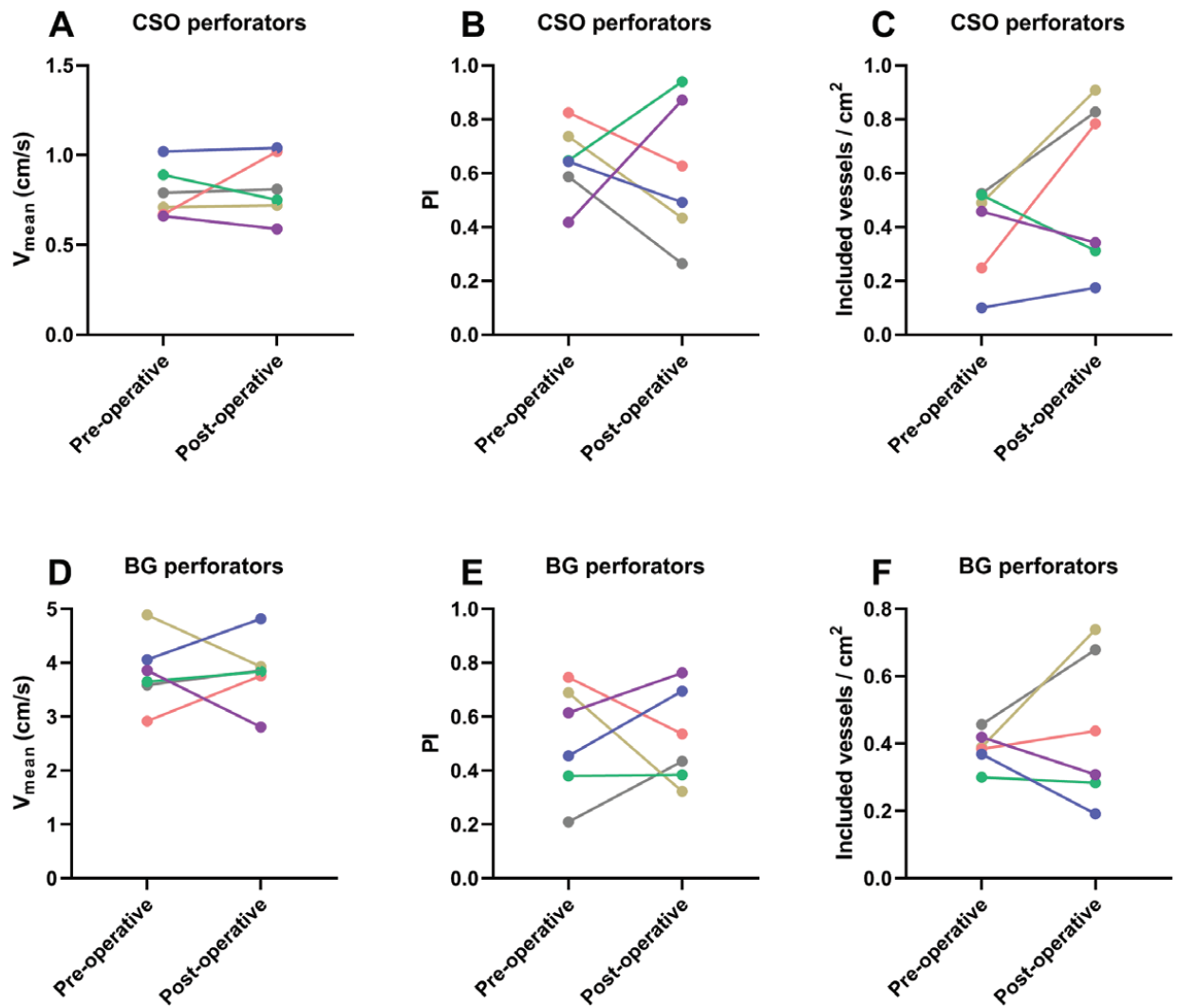

Figure 3 Results of the ipsilateral CSO and BG perforators. For each of the patients the post-operative values are plotted against pre-operative values. Panels $A, B$ and $C$ represent respectively ipsilateral Vmean, pulsatility index (PI) and number of included perforators/cm2 for the ipsilateral CSO and panels D, E and $\mathrm{F}$ for the BG perforators. A considerable large increase in the Vmean of the CSO perforators is visible in one of the patients (orange line $=$ patient 3 ; increase of $153 \%$ ) while increase of Vmean in other patients varied between $-15 \%$ and $+2 \%$. Change in Vmean of the BG perforators varied between $-27 \%$ and $+29 \%$. Change in $\mathrm{PI}$ of the CSO perforators between $-55 \%$ and $+109 \%$ and in the BG perforators between $-26 \%$ and $+113 \%$. Change of CSO perforators $/ \mathrm{cm} 2$ varied between $-40 \%$ and $+216 \%$ and between $-48 \%$ and $+90 \%$ in BG perforators. 


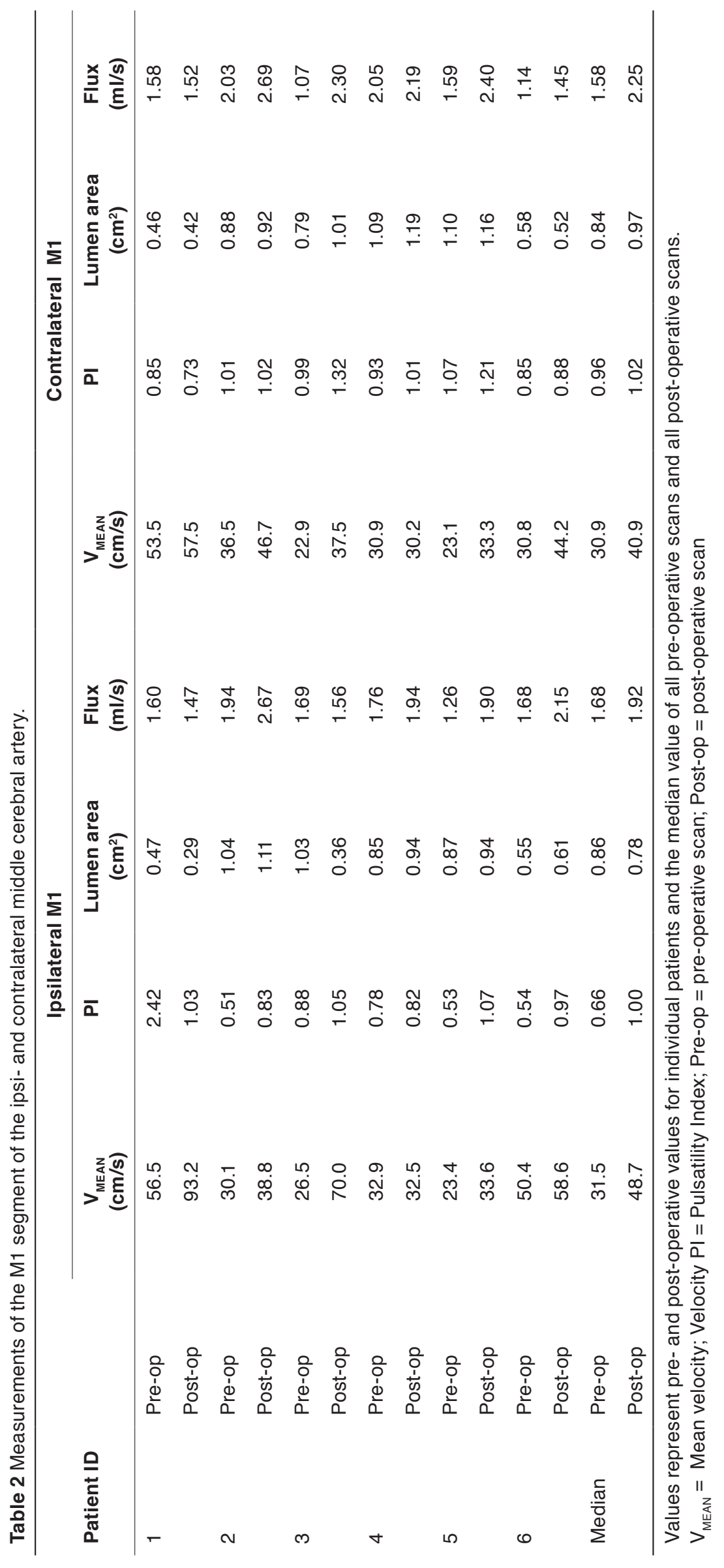




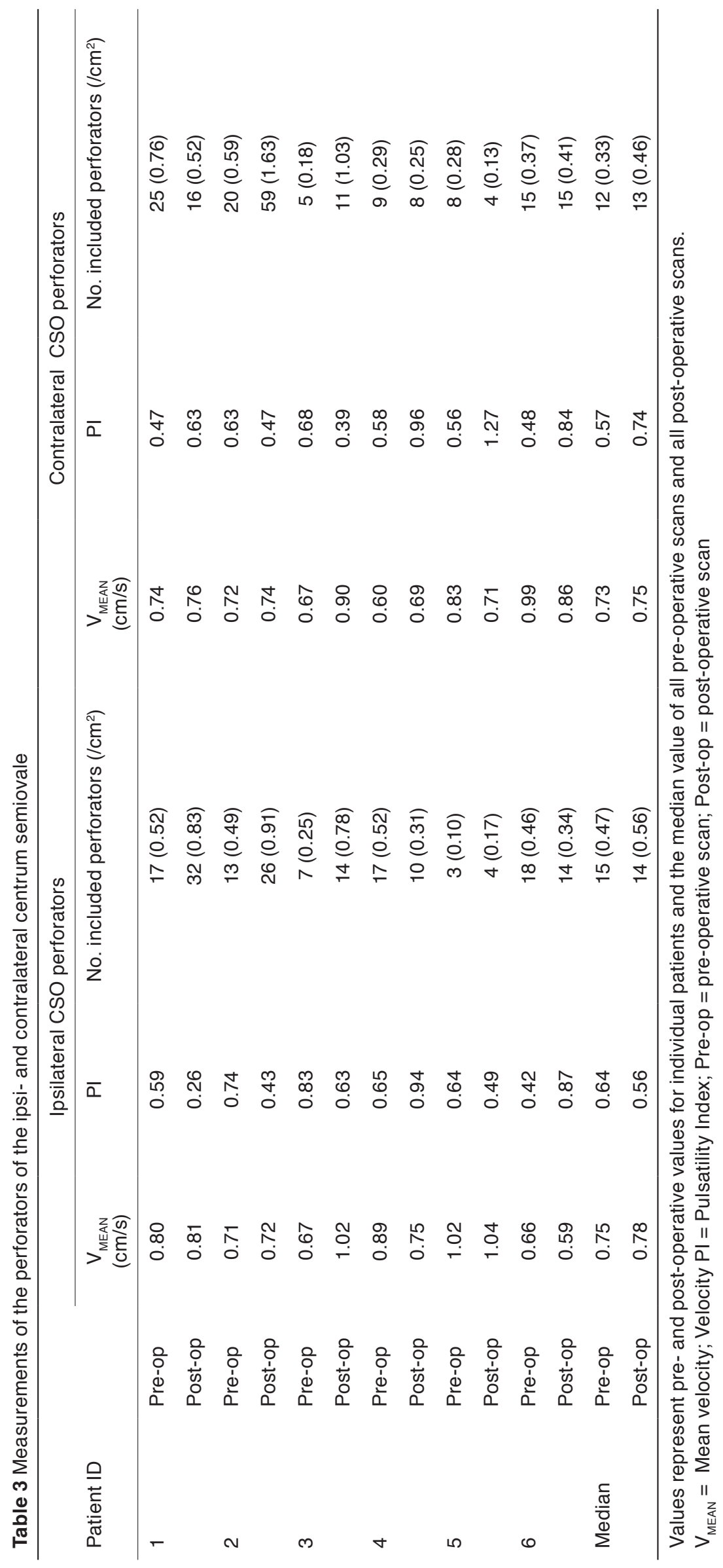




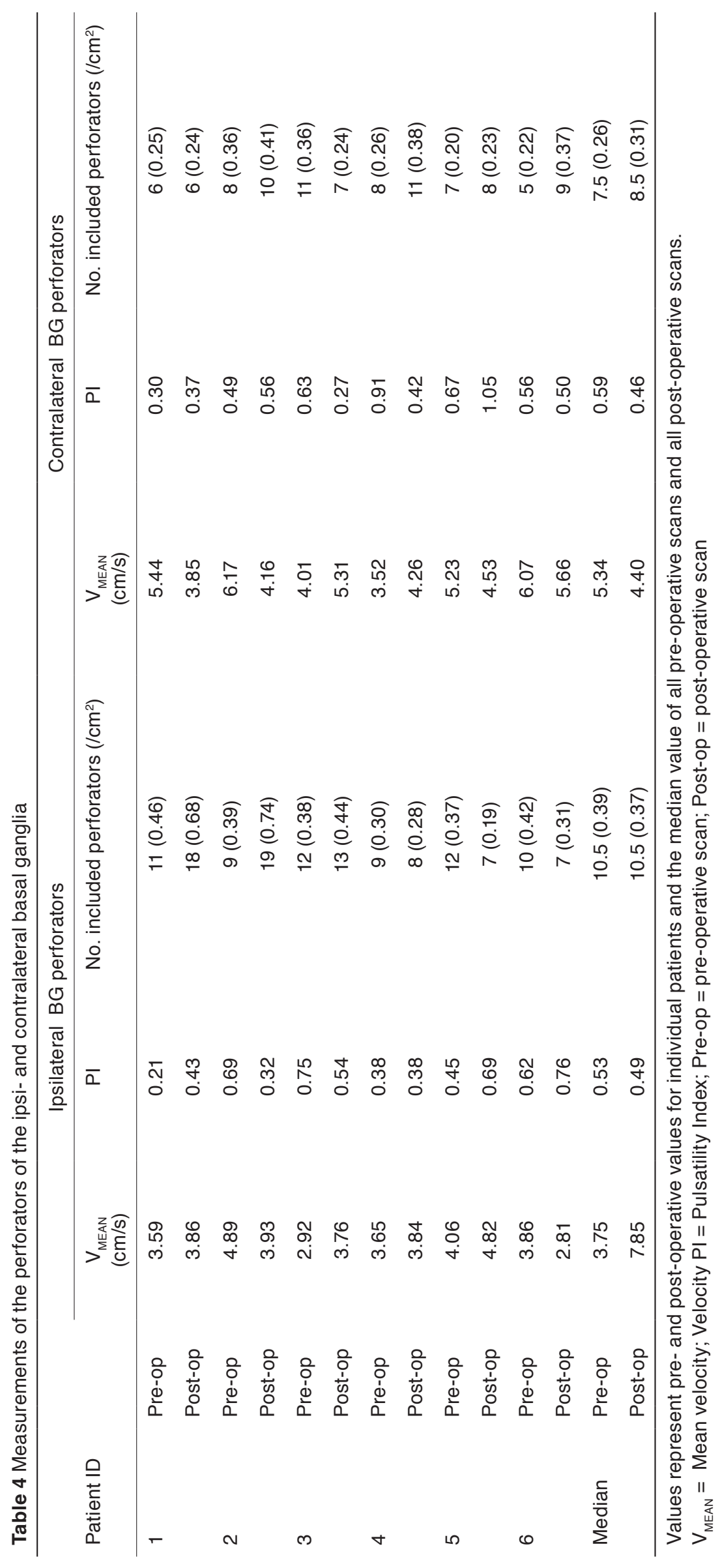




\section{BG}

A median of 18 (range 15-29) BG perforators were identified in each of the 12 scans with a median of 10 ipsilateral and 8 contralateral perforators. In table 4 the tested parameters of the post-operative as compared to pre-operative ipsi- and contralateral BG perforators are depicted. Median baseline $\mathrm{V}_{\text {mean }}$ was 3.75 (range $2.92-4.89$ ) on the ipsilateral side and 5.34 (range $3.51-6.17$ ) on the contralateral side. Change in ipsilateral $\mathrm{V}_{\text {mean }}$ of the $B G$ perforators was moderate and varied between $-27 \%$ and $+29 \%$ (figure 3 , panel D) which was comparable to the contralateral side (between $-33 \%$ and $+32 \%$ ). On pre-operative scan median PI of the ipsilateral BG perforators was 0.53 (range $0.2-0.75$ ), median $\mathrm{PI}$ of the contralateral BG perforators was 0.59 (range $0.30-0.91$ ). Post-operative ipsilateral change of $\mathrm{PI}$ varied between $-33 \%$ and $+112 \%$ (figure 3 , panel $\mathrm{E}$ ) as compared to $-57 \%$ and $+58 \%$ on the contralateral side. Median number of perforators included per $\mathrm{cm}^{2} \mathrm{BG}$-mask at baseline was $0.39(0.30-0.56)$ on the ipsilsateral side and $0.26(0.20-0.36)$ on the contralateral side. Change of post-operative scan compared to pre-operative scan varied between $-48 \%$ and $+89 \%$ on the ipsilateral sided (figure 3 panel $\mathrm{F}$ ) and $-33 \%$ and $+68 \%$ on the contralateral side.

\section{CoW}

All patients showed completeness of the anterior portion of the CoW, with one patient (patient

3) having reversed flow in the A1 segment of the ipsilateral anterior cerebral artery on the pre-operative scan. CoW flow was normalized on the post-operative scan in this patient.

\section{Secondary outcome measures}

No relevant changes in BP were present at the moment of post-operative MRI compared to pre-operative MRI (supplemental table S1). In five out of six patients TCD monitoring was possible, in one patient (patient 3 ) temporal bone window was inadequate (supplemental table S1). On TCD none of the five patients had an increase of MCA velocity of $\geq 100 \%$ and none of the patients experienced CHS. No periprocedural stroke occurred.

\section{Discussion}

In this explorative study we investigated the effect of a revascularisation procedure of the internal carotid artery on large and small cerebral arterial function as measured by 7T MRI. We found that imaging of the large cerebral arteries as well as the perforators is feasible in patients prior to and after CEA. As a result of the procedure, we demonstrated that changes in velocity and pulsatility of the M1 did not necessarily result in a similar change in flux (perfusion), nor in a similar response in CSO and BP perforators and that both the direction as well as the magnitude of these changes varied widely between patients.

In carotid artery disease, cerebral blood flow may be compromised when autoregulatory mechanisms are exhausted and/or collateral circulation is incompetent and unable to compensate the reduction in cerebral perfusion pressure ${ }^{18-21}$. Under these conditions, patients may benefit from a carotid revascularisation in terms of haemodynamic improvement although the effect of such a procedure on perforator arterial function still needs to be established. 
It is well known that changes in blood flow velocity in the MCA occur as a result of a revascularisation procedure as this is registered by TCD in daily clinical practice ${ }^{22,23}$. We demonstrated that the relation between MCA velocity and MCA flux as well as perforator function is complex and the effect of carotid intervention on cerebral arterial function is ambiguous.

The lack of effect on CSO or BG perforator velocity in some of the patients may be explained by the considerably unaffected contralateral carotid arteries of the included patients with none of them having a contralateral high-grade stenosis or occlusion. This is in agreement with our experience among patients with unilateral carotid artery occlusion that do not have a significantly different cerebral arterial perforator function compared the contralateral hemisphere or to healthy controls (unpublished data). Furthermore, in this study no incompleteness of the anterior part of the CoW was observed and thus important collateral pathways were adequate in these patients. One patient did show reversed flow in the $\mathrm{A} 1$ segment of the ipsilateral anterior cerebral artery on preoperative imaging, indicating incompetent compensatory mechanisms, which appeared to be normalised after CEA. This was the only patient that showed a great increase in $\mathrm{V}_{\text {mean }}$ of the perforators and additionally showed an increase in the number of detected perforators. This may imply that patients with exhausted or incompetent cerebral autoregulation and/or collateral circulation may benefit from revascularisation for improvement of cerebral perforator arterial function. This is in agreement with studies showing similar results in patients with exhausted cerebrovascular reserve (CVR) ${ }^{24}$. However, CVR merely reflects the ability of the cerebrovascular system to respond to a vasodilatory challenge while for the first time we have demonstrated the effect of a carotid revascularisation on the perforating arterial function, including pulsatility data. Carotid artery disease may influence PI of the cerebral arteries by several mechanisms. First of all, calcified lesions in proximal arteries such as the carotid artery or aortic arch, may facilitate transmission of pulsatile energy in the cerebral microcirculation due to impaired cushioning function ${ }^{9,25}$. Excessive power in the cerebral perforators as a result of increased pulsatility may damage vessel walls and lead to remodelling and tearing of endothelial and smooth muscle cells ${ }^{9}$. This theory is supported by literature showing that local carotid stiffness is associated with white matter lesions (WML), lacunar infarction and cerebral microbleeds, all radiographic expressions of cerebral small vessel disease ${ }^{9,10}$. On the other hand, when blood flow through the carotid artery is restricted due to a high-grade stenosis, transmission of energy may be hampered due to the drop of pressure gradient ${ }^{26}$. In addition, in patients with severe ICA stenosis, cerebrovascular resistance may be decreased through autoregulatory mechanisms in order to maintain adequate cerebral blood flow (CBF), in its turn decreasing cerebral artery pulsatility ${ }^{27}$. Decreased pulsatility as a result of carotid ligation in an animal model resulted in decreased exchange between the cerebrospinal fluid and interstitial fluid ${ }^{28}$ which may contribute to neurodegenerative processes as a results of hampering of clearance of metabolic waste products and solutes ${ }^{29}$. Our results showed a clear decrease of MCA pulsatility in one patient with high pre-operative PI, which may be explained by removal of the stiff atherosclerotic plaque. MCA PI in other patients increased, probably due to improved flow in the operated artery. Post-operative MCA PI values were all in the same range so 
endarterectomy appears to result in a normalisation of pulsatility in the MCA. Yet, these changes in MCA PI were not reflected by equivalent shifts in pulsatility of the perforating arteries in CSO and BG. Interaction of cerebrovascular autoregulatory mechanisms may play an important role and as such prediction of the effect of a revascularisation procedure on the arterial pulsatility in the cerebral microcirculation is challenging. Moreover, the relation between perforating artery pulsatility and the effect of a carotid artery stenting should be a topic for future research as an endovascular stent may increase carotid stiffness and thereby affect perforating artery pulsatility. This topic is especially relevant since novel stenting techniques such as TCAR (transcarotid artery revascularisation) are being used progressively ${ }^{30}$.

Another possible applicability of this imaging technique and the findings presented is to identify those at risk for CHS. Patients are at risk for $\mathrm{CHS}$ when regional hyperaemia after plaque removal overwhelms autoregulatory mechanisms ${ }^{31}$. Inadequacy of the cerebrovascular system to correct for these changes may be reflected by changes in $\mathrm{V}_{\text {mean }}$ of the perforating arteries rather than the $\mathrm{V}_{\text {mean }}$ of the MCA. One of the patients included in our study (patient 3) showed a large increase in $V_{\text {mean }}$ and number of detected CSO and BG perforators while MCA lumen-area had decreased remarkably, suggesting insufficient ability within the autoregulatory mechanisms to compensate for the sudden change in ipsilateral carotid artery blood flow. Notably, patients with exhausted autoregulatory mechanisms may benefit most from a revascularisation but may also be the ones most at risk for $\mathrm{CHS}$ since prolonged exposure to ischaemic conditions damages vascular smooth muscle cells making them unable to dilate or constrict in response to stimuli ${ }^{32}$. Increased velocities in the perforating arteries may be an indicator that can help identifying patients at risk for CHS. TCD has proven to be a reliable technique for identifying those not at risk for $\mathrm{CHS}^{23}$ but overtreatment with the accompanied high hospital coasts as a result of continuous BP registration and prolonged hospital admittance is common ${ }^{12}$. Moreover, $5-37 \%$ of patients have no adequate acoustic temporal bone-window ${ }^{33}$, as was the case in one of our patients, therefore lacking appropriate monitoring. Possibly further risk assessment using non-invasive MRI technologies may offer a solution to this problem by successive screening of patients with increased TCD velocities or those with inadequate temporal bone window.

\section{Limitations}

This study has several limitations. Firstly, post-operative MRI was made one day after CEA and we have no information on the long-term effect on cerebral perforator arterial function. In the vast majority of patients cerebral hyperperfusion occurs in the first 7 days after the procedure $^{34}$ so it is likely that post-procedural changes observed in our patients negated over the following days. Follow-up studies need to assess the long-term effects on cerebral arterial function, although for identification of patients at risk for $\mathrm{CHS}$ the periprocedural period is most relevant. Secondly, larger studies are required to be able to make any statistical comparison between pre-operative and post-operative data. Third, some of the observed differences may be a reflection of measurements errors, although earlier studies using this method showed

good reproducibility ${ }^{13}$. Clear difference in $\mathrm{V}_{\text {mean }}$ of the CSO was observed in one patient (figure 3 panel A) and this difference $(0.3553 \mathrm{~cm} / \mathrm{s})$ was much larger than the mean absolute 
difference seen in $\mathrm{V}_{\text {mean }}$ in repeated scans in the same patients in this reproducibility study $\left(0.14 \mathrm{~cm} / \mathrm{s}^{13}\right)$. For the $V_{\text {mean }}$ of the $B G$ a clear trend can be seen in four out of six patients (figure 3, panel D), two of them larger than the mean absolute difference seen in $\mathrm{V}_{\text {mean }}$ in repeated scans in the same patients $\left(0.66 \mathrm{~cm} / \mathrm{s}^{13}\right)$. Changes in $\mathrm{PI}$ exceeded measurement errors as previously reported in all patients for CSO perforators and all but one patient for BG perforators. At last, a threshold $\mathrm{V}_{\text {mean }}$ value was used for selecting the perforators included for further analysis. This might have blurred any effect on the $V_{\text {mean }}$ values and these should therefore be interpreted in combination with the numbers of detected values.

\section{Conclusion}

In this exploratory study we investigated the feasibility of ultra-high field MR imaging to evaluate the effect of carotid revascularisation procedure on cerebral arterial function. We demonstrated that the relation between large and perforating artery velocity, flow and pulsatility is complex and ambiguous. Future studies should confirm whether these novel imaging techniques can be deployed for identification of patients that will benefit from carotid revascularisation and those at risk for cerebral hyperperfusion syndrome.

\section{Acknowledgements}

We thank J.J. Sloots and K.M. van Hespen for their contributions in MRI data acquisition and analysis. 


\section{References}

1. Group ECSTC. Randomised trial of endarterectomy for recently symptomatic carotid stenosis: final results of the MRC European Carotid Surgery Trial (ECST). 1998;351(9113):1379-87.

2. Ederle J, Davagnanam I, van der Worp HB, Venables GS, Lyrer PA, Featherstone RL, et al. Effect of whitematter lesions on the risk of periprocedural stroke after carotid artery stenting versus endarterectomy in the International Carotid Stenting Study (ICSS): A prespecified analysis of data from a randomised trial. Lancet Neurol. 2013;12(9):866-72.

3. Bonati LH, Jongen LM, Haller S, Flach HZ, Dobson J, Nederkoorn PJ, et al. New ischaemic brain lesions on MRI after stenting or endarterectomy for symptomatic carotid stenosis: a substudy of the International Carotid Stenting Study (ICSS). Lancet Neurol. 2010 Apr;9(4):353-62.

4. Gaba K, Ringleb PA, Halliday A. Asymptomatic Carotid Stenosis: Intervention or Best Medical Therapy? Curr Neurol Neurosci Rep. 2018;18(11):1-9.

5. Mortimer R, Machiappan S, Howlett DC. Carotid artery stenosis screening: Where are we now? Br J Radiol. 2018;91(1090).

6. Duncombe J, Kitamura A, Hase Y, Ihara M, Kalaria R, Horsburgh K. Chronic cerebral hypoperfusion: a key mechanism leading to vascular cognitive impairment and dementia (VCID). Closing the translational gap between rodent models and human VCID. Clin Sci. 2017;131(19):2451-68.

7. Klijn CJM, Kappelle LJ, Tulleken CAF, van Gijn JM. Symptomatic Carotid Artery Occlusion: A Reappraisal of Hemodynamic Factors. Stroke. 1997;28(10):2084-2093.

8. Mitchell GF. Aortic stiffness, pressure and flow pulsatility, and target organ damage. J Appl Physiol. 2018;125(6):1871-80.

9. Ding J, Mitchell GF, Bots ML, Sigurdsson S, Harris TB, Garcia M, et al. Carotid Arterial Stiffness and Risk of Incident Cerebral Microbleeds in Older People: the AGES-Reykjavik Study. Arter Thromb Vascu Biol. 2015;35(8):1889-95.

10. Brisset M, Boutouyrie P, Pico F, Zhu Y, Zureik M, Schilling S, et al. Large-vessel correlates of cerebral small-vessel disease. Neurology. 2013;80(7):662-9.

11. Pennekamp CWA, Tromp SC, Ackerstaff RGA, Bots ML, Immink R V., Spiering W, et al. Prediction of cerebral hyperperfusion after carotid endarterectomy with transcranial Doppler. Eur J Vasc Endovasc Surg. 2012;43(4):371-6.

12. Dalman JE, Beenakkers ICM, Moll FL, Leusink JA, R.G.A. Ackerstaff. Transcranial Doppler monitoring during carotid endarterectomy helps to identify patients at risk of postoperative hyperperfusion. Eur J Vasc Endovasc Surg. 1999;18(3):222-7.

13. Bouvy WH, Geurts LJ, Kuijf HJ, Luijten PR, Kappelle LJ, Biessels GJ, et al. Assessment of blood flow velocity and pulsatility in cerebral perforating arteries with 7-T quantitative flow MRI. NMR Biomed. 2016;29(9):1295-304.

14. Geurts L, Biessels GJ, Luijten P, Zwanenburg J. Better and faster velocity pulsatility assessment in cerebral white matter perforating arteries with 7T quantitative flow MRI through improved slice profile, acquisition scheme, and postprocessing. Magn Reson Med. 2018;79(3):1473-82.

15. Ferguson GG, Eliasziw M, Barr HWK, Clagett GP, Barnes RW, Wallace MC, et al. The North American Symptomatic Carotid Endarterectomy Trial Surgical Surgical Results in 1415 Patients. Stroke. 2015;30:1751-8.

16. Goldstein RM, Zebker HA, Werner CL. Satellite radar interferometry: Two-dimensional phase unwrapping. Radio Sci. 1988;23(4):713-20.

17. Arts T, Siero J, Biessels G, Zwanenburg J. Method for vessel selection effects the outcome and reproducibility of velocity and pulsatility measures in cerebral penetrating arteries. Annu Meet Int Soc Magn Reson Montr. 2019;\#3264.

18. Landesberg G, Adam D, Berlatzky Y, Akselrod S. Step baroreflex response in awake patients undergoing carotid surgery: time- and frequency-domain analysis. Am J Physiol Circ Physiol. 2017;274(5):H1590-7.

19. Romero JR, Pikula A, Nguyen TN, Nien YL, Norbash A, Babikian VL. Cerebral Collateral Circulation in Carotid Artery Disease. Curr Cardiol Rev. 2010;5(4):279-88.

20. Powers WJ. Cerebral hemodynamics in ischemic cerebrovascular disease. Ann Neurol. 1991;29(3):231-40.

21. Nasr N, Czosnyka M, Pavy-Le Traon A, Custaud M-A, Liu X, Varsos G V, et al. Baroreflex and cerebral autoregulation are inversely correlated. Circ J. 2014;78(10):2460-7.

22. Pennekamp CWA, van Laar PJ, Hendrikse J, den Ruijter HM, Bots ML, van der Worp HB, et al. Incompleteness of the circle of Willis is related to EEG-based shunting during carotid endarterectomy. Eur J Vasc Endovasc Surg. 2013 Dec;46(6):631-7.

23. Fassaert LMM, Immink R V., van Vriesland DJ, de Vries J-PPM, Toorop RJ, Kappelle LJ, et al. Transcranial Doppler 24 Hours after Carotid Endarterectomy Accurately Identifies Patients Not at Risk of Cerebral Hyperperfusion Syndrome. Eur J Vasc Endovasc Surg. 2019;58(3):320-7. 
24. Chang TY, Liu HL, Lee TH, Kuan WC, Chang $\mathrm{CH}$, Wu HC, et al. Change in cerebral perfusion after carotid angioplasty with stenting is related to cerebral vasoreactivity: A study using dynamic susceptibilityweighted contrast-enhanced MR imaging and functional MR imaging with a breath-holding paradigm. Am J Neuroradiol. 2009;30(7):1330-6.

25. Mitchell GF, Van Buchem MA, Sigurdsson S, Gotal JD, Jonsdottir MK, Kjartansson Ó, et al. Arterial stiffness, pressure and flow pulsatility and brain structure and function: The Age, Gene/Environment Susceptibility-Reykjavik Study. Brain. 2011;134(11):3398-407.

26. Liu X, Zhang H, Ren L, Xiong H, Gao Z, Xu P, et al. Functional assessment of the stenotic carotid artery by cfd-based pressure gradient evaluation. Am J Physiol - Hear Circ Physiol. 2016;311(3):H645-53.

27. Duffin J, Sobczyk O, McKetton L, Crawley A, Poublanc J, Venkatraghavan L, et al. Cerebrovascular resistance: The basis of cerebrovascular reactivity. Front Neurosci. 2018;12(JUN):1-14.

28. Iliff JJ, Wang M, Zeppenfeld DM, Venkataraman A, Plog BA, Liao Y, et al. Cerebral arterial pulsation drives paravascular CSF-Interstitial fluid exchange in the murine brain. J Neurosci. 2013;33(46):18190-9.

29. Bacyinski A, Xu M, Wang W, Hu J. The paravascular pathway for brain waste clearance: Current understanding, significance and controversy. Front Neuroanat. 2017;11(November):1-8.

30. LIANG P, WU WW, SCHERMERHORN ML. Recent advances in the treatment of carotid artery disease. J Cardiovasc Surg (Torino). 2019;60(3):345-53.

31. Kirchoff-Torres KF, Bakradze E. Cerebral Hyperperfusion Syndrome After Carotid Revascularization and Acute Ischemic Stroke. Curr Pain Headache Rep. 2018;22(4).

32. Lieb M, Shah U, Hines GL. Cerebral hyperperfusion syndrome after carotid intervention: A review. Cardiol Rev. 2012;20(2):84-9.

33. Wijnhoud AD, Franckena M, van der Lugt A, Koudstaal PJ, Dippel en DWJ. Inadequate Acoustical Temporal Bone Window in Patients with a Transient Ischemic Attack or Minor Stroke: Role of Skull Thickness and Bone Density. Ultrasound Med Biol. 2008;34(6):923-9.

34. Bouri S, Thapar A, Shalhoub J, Jayasooriya G, Fernando A, Franklin IJ, et al. Hypertension and the postcarotid endarterectomy cerebral hyperperfusion syndrome. Eur J Vasc Endovasc Surg. 2011;41(2):229-37. 


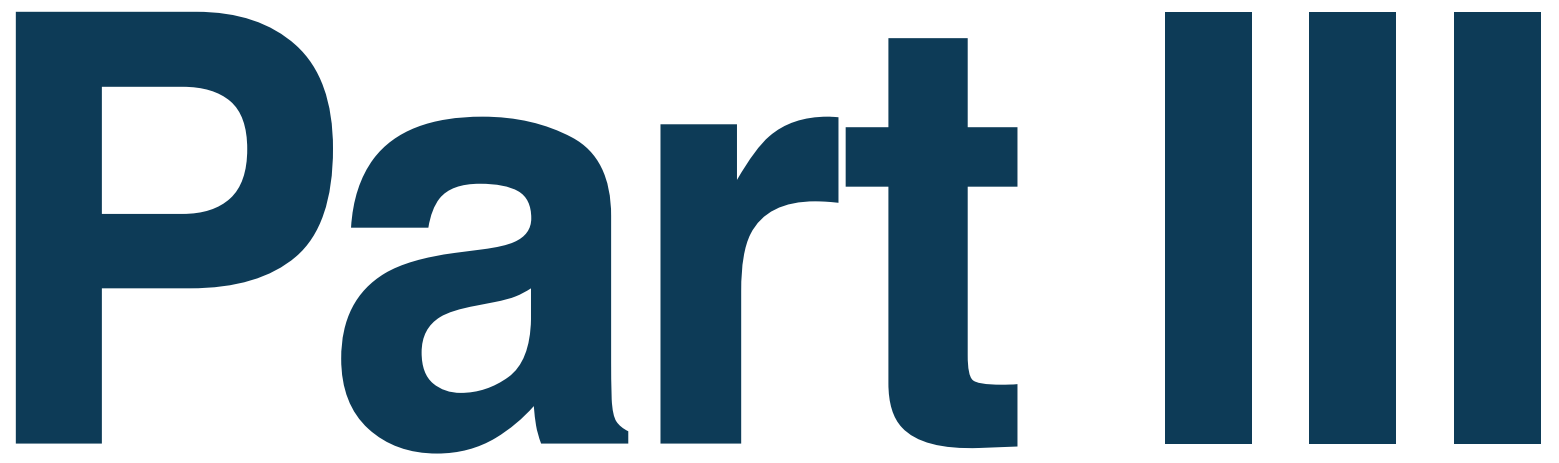

Summarizing discussion, future perspectives and conclusion 

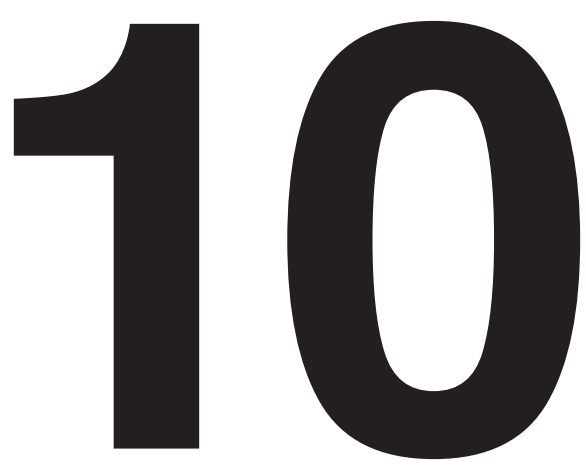

Summarizing discussion, future perspectives and conclusion 


\section{Summarizing discussion and future perspectives}

This thesis describes various aspects of assessing periprocedural outcome in patients treated for carotid artery disease; it discusses imaging markers that can be used for determining outcome as a surrogate measure for stroke as well as the effect of revascularisation procedures on cerebral haemodynamics. In this thesis we aimed to explore novel methods that may eventually lead to improved patient- and treatment selection, with the ultimate goal to progress individualized choice of treatment strategy for carotid artery disease. This chapter provides a general discussion and summary of all presented studies including the therefrom following future perspectives and challenges.

\section{A shifting paradigm; from classical endpoints to surrogate outcome markers}

Over the years, optimization of medical therapy, lifestyle improvements and advances in surgical and endovascular techniques have improved the periprocedural and long term risk rates of patients with carotid artery disease ${ }^{1}$. Alongside these positive developments, it has become increasingly challenging to detect positive (or negative) effects of novel diagnostic or therapeutic algorithms on classical endpoints such as "procedural stroke and death" and "recurrent stroke". Large numbers of patients are required to find a significant effect of intervention on these clinical endpoints. In chapter 2 , we describe the most wellknown surrogate markers for outcome and we argue that these surrogate markers should be implemented in daily clinical practice. Diffusion weighted imaging (DWI) lesions, silent brain infarcts (SBIs) and white matter lesions (WMLs) are promising and complementary surrogate markers ${ }^{2}$. Standardization of imaging protocols and reporting of outcome is highly desired. In our view, pre- and post-procedural brain MRI has to be implemented in standard care to encourage and facilitate employment of these surrogate markers in research and clinical practice when treating patients with carotid artery disease. Future studies should focus on long-term outcome of patients with manifestation of these surrogate outcome markers to further explore their clinical relevance. DWI lesions are considered to be reliable surrogate markers as they have a biological plausible relationship with stroke ${ }^{3}$, they are associated with increased risk of postprocedural stroke/TIA ${ }^{4}$ and the treatment effects on DWI-lesions and stroke are correlated ${ }^{5}$. However, although DWI lesions have been associated with increased risk of future stroke/TIA after carotid artery stenting (CAS) ${ }^{4}$ and non-cardiac surgery in general ${ }^{6}$, such a correlation has not yet been demonstrated for patients after carotid endarterectomy. The lack thereof may be explained by the fact that the incidence of new lesions is much lower after $\mathrm{CEA}^{7}$ and follow-up studies were insufficiently powered to find a significant association. Furthermore, the follow-up period may have been insufficient (median of 4.1 years) to detect ample events to find any correlation ${ }^{4}$. In our opinion, implementation of standard imaging in clinical care and registration of long-term outcome as part of clinical registries can help to answer these questions. Irrefutable evidence affirming the clinical relevance of DWI lesions after a carotid revascularization procedure is of major importance in the process towards using these imaging surrogate markers as a primary endpoint in research and clinical practice. 


\section{Individualizing optimal treatment strategy}

Surrogate outcome markers can help to identify patients at risk for periprocedural complications. Assessment of patient specific features can be useful in selecting the best treatment modality, either invasive or conservative, based on individualized risk scores. Chapter 3 systematically reviewed various patient characteristics that contribute to the periprocedural risk of new DWI lesions after either CEA or CAS. These risk factors appeared to be heterogeneous and quite dissimilar for both treatment options, which may well be explained by different mechanisms of cerebral ischaemia during both procedures. DWI lesions may develop as a result of either haemodynamic, thrombo-embolic or a combination of both mechanisms (impaired clearance of emboli) ${ }^{8}$. Plaque instability or presence of proximal lesions (in the common carotid artery or aortic arch) may be a problem in stenting rather than during CEA, whereas insufficient cerebrovascular reserve or poor haemodynamic conditions may be more problematic during cross-clamping while performing CEA. For example; a relatively young patient with poor cerebrovascular reserve may benefit from CAS rather than CEA while an octogenarian with several proximal lesions in the aortic arch and signs of instability of the culprit lesion will probably benefit most from a surgical procedure. This theory is supported by the dissimilarity in risk factors for both procedures as found in this chapter ${ }^{9}$. However, up to now no comparative studies have been performed that prove that one specific procedure or treatment algorithm may be more beneficial in a patient with a certain 'risk profile'. Future research should focus on developing clinical decision making-algorithms that include multiple patient related risk factors ${ }^{10}$. A national or international carotid disease data registry, in which imaging, laboratory and demographic features as well as the chosen treatment regime are accurately listed, may be valuable for this purpose. Prospective observational studies are needed to assess postprocedural DWI lesions, while such decision-making algorithms are deployed for choosing the optimal treatment based on patient-related features. As mentioned earlier, DWI lesions are reliable surrogate markers and they occur more frequently than classical endpoints. Another advantage of DWI lesions is that they may appear within several hours after a thrombo-embolic event, making this imaging modality sensitive to recent changes. In combination with apparent diffusion coefficient (ADC)-maps, which may depict darkening within minutes of stroke onset and distinguishes stroke from "T2 shine through", lesions can be considered to be recent (about 7-10 days ${ }^{11}$ ) and can be differentiated from older infarcts. DWI lesions can therefore also be used to assess recurrent events in previously symptomatic patients (up until revascularisation) rather than only as a surrogate marker for postprocedural outcome. This is demonstrated in chapter 4. We investigated carotid intraplaque haemorrhage (IPH) as a potential source of recurrent brain ischaemia in the waiting time between index event (or hospital presentation) and CEA. We found that previously symptomatic patients in which preoperative MR-DWI showed signs of recurrent cerebral ischaemia, more often had histologically apparent carotid plaque IPH compared to patients without signs of recurrent cerebral ischaemia. This stresses the importance of eliminating an unstable plaque prior to any (clinical or subclinical) recurrent event. This is relevant especially since a broad consensus on early versus expedited treatment is still lacking $^{12-15}$. Assessment of IPH can help to prioritize those that may benefit more from a 
rapid revascularisation procedure. Our study showed that this also applies in case of a preor in-hospital delay of $>14$ days after the index event. Delay of more than 14 days should not withhold treating physicians to offer a revascularisation procedure, especially when the plaque shows signs of instability such as IPH. Reliable and easily accessible methods for preoperative establishment of IPH on imaging is essential for clinical use. So far, radiographic studies showed good agreement with histological data ${ }^{16}$ but further development of imaging techniques for IPH detection is warranted in case of smaller haemorrhage or coexisting calcifications ${ }^{16}$.

\section{Baseline brain and carotid artery imaging for prediction of outcome}

In carotid artery disease patients, baseline brain imaging is often performed to assess the presence and extensiveness of any ischaemic lesions as part of standard clinical workup. These baseline brain images also comprise other valuable information such as presence and severity of small vessel disease. Small vessel disease, as measured by white matter lesions on $\mathrm{CT}$ or $\mathrm{MRI}$, has previously been suggested as a potential predictor for perioperative adverse events following CEA ${ }^{17}$. In chapter $\mathbf{5}$, we demonstrated that mentioning of SVD in standard brain imaging also seems to be associated with a generally increased risk of long-term cardiovascular death for symptomatic patients undergoing CEA. Yet, presence of preoperative SVD was not associated with increased risk of stroke (either 30-day or long-term risk). This finding is especially relevant since improved preventive medical strategies have decreased recurrent stroke-risk and consequently the number-needed-to-treat may have increased over the years. As such, the benefit of a revascularisation procedure may be relatively smaller in patients with a poorer long-term prognosis based on extensive preoperative SVD, which may alter the balance towards choosing a conservative treatment strategy. A randomized trial currently running (the European Carotid Surgery Trial 2, ECST-2) investigates conservative versus invasive therapy in 'low-risk' asymptomatic or symptomatic patients. The ECST-2 uses a carotid stenosis risk prediction too ${ }^{18}$ for assessment of risk at 5 -year ipsilateral stroke and intends to validate this score based on their study results ${ }^{19}$. Study results discussed in chapter 5 of this thesis suggest that risk models intended for choosing between conservative and invasive therapy may benefit from including baseline MR imaging. Future risk stratification algorithms should include multiple demographic, biological and imaging characteristics for decision making on optimal treatment strategy. Large datasets are required to design and subsequently validate such models, and we argue that assessment of SVD should become part of standard clinical workup to facilitate this.

In chapter $\mathbf{6}$ we used MR imaging of the carotid wall in patients with an extracranial carotid artery aneurysm (ECAA) to see whether baseline imaging correlates with aneurysm growth or cerebral complications. In this explorative study we investigated whether aneurysm vessel wall imaging was feasible and whether contrast enhancement with Gadolium (Gd) was related to outcome. So far, very little is known regarding the best treatment strategy for extracranial aneurysms in asymptomatic patients. We found that the majority of patients showed aneurysm wall-enhancement at baseline, while aneurysm growth occurred in about a quarter of all patients (both with and without wall-enhancement). Thus, although aneurysm wall imaging 
was feasible with good inter- and intra-observer reliability and agreement, the current study design did not result in evincive evidence that Gd-enhancement is related to ECAA instability. The study described in this thesis was based on protocols used for intracranial aneurysms, in which wall enhancement appeared to be a good predictor for growth ${ }^{20}$. However, vessel wall anatomy may differ between arteries, such as the presence of an adventitial vasovasorum in extracranial arteries as opposed to healthy intracranial arteries ${ }^{21,22}$. This may complicate the ability to differentiate physiological from pathological vessel wall enhancement in extracranial arteries. Furthermore, 'slow flow' in these aneurysms may be incorrectly qualified as vessel wall enhancement and improvement in scanning sequences for ECAA wall imaging should be strived for. The next step for research on this topic may be to initiate a study in both healthy volunteers and ECAA patients in which optimal dosage and flow rate of the contrast agent for differentiation between healthy and aneurysmatic carotid wall is being determined. Development of (semi-)automatic quantitative methods for determining the distribution and extensiveness of vessel wall enhancement might help in reliably assessing the level of enhancement. One could hypothesize that slight enhancement of the carotid wall is physiological while severe enhancement is pathological and associated with aneurysm instability ${ }^{20}$. Making a quantitative discrimination may help to further crystallize which exact features should be investigated in relation to cerebrovascular complications of ECAAs. Subsequent well-powered studies are needed to further investigate whether Gd-enhancement can identify patients at risk for aneurysm growth and/or thrombo-embolic complications, and ideally whether screening of these features can help in deciding between conservative or invasive treatment strategies. The currently running carotid aneurysm registry $(C A R)^{23}$ provides a large database of patients with this considerably rare disease and can be a source for patient recruiting for such a study. Ideally, such studies yield radiographical risk markers that can assist in determining treatment approaches so patients at risk can undergo surgery prior to any thrombo-embolic complications. Also, the intensity of follow-up imaging can be scaled back in patients that benefit most from a conservative approach.

\section{Cerebral haemodynamics during carotid endarterectomy}

During carotid endarterectomy, cerebral haemodynamics should be optimized in order to maintain sufficient cerebral perfusion. Especially in de cross-clamping phase of the procedure, when cerebral perfusion on the operated side is dependent on contralateral blood flow, sustaining adequate systemic blood pressure (BP) by appropriate use of vasoactive agents is essential. In chapter $\mathbf{7}$ we investigated whether intraoperative drops in BP compared to baseline BP is related to an increased risk for periprocedural DWI lesions. We found that BP drops were larger in DWI-positive patients compared to DWI-negative patients. These results suggest that suboptimal perioperative haemodynamics may contribute to the development of cerebral complications after CEA. One of the challenges in this topic is to determine the most representative baseline BP that can be used as a basis to set a peri-operative target BP. Preinduction $\mathrm{BP}$ has appeared to be non- representative for a patient's true $\mathrm{BP}^{24}$, but $\mathrm{BP}$ values measured at intake or hospital admission may be high due to white coat hypertension as well ${ }^{25}$. Future studies should structurally record and report both preoperative and pre-induction BP as 
a baseline value. A trial randomizing between hypertension and normotension can be helpful for determining optimal treatment strategy during carotid procedures. Intraoperative hyperand normotension should be defined as a percentage compared to baseline values, using a mean of structurally measured preoperative values. Again, reporting of all preoperative and pre-induction BP values in such a trial is essential. Another challenge regarding this topic is the optimal strategy for preoperative normalization of hypertensive BP. Although systolic $\mathrm{BP}>180 \mathrm{mmHg}$ has been independently associated with an increased risk of stroke after $\mathrm{CEA}^{26}$, it is unclear whether lowering this $\mathrm{BP}$ in the preoperative period reduces the risk of events. Finally, although haemodynamics have been an important topic for CEA procedures, this is not so much the case for CAS, in which haemodynamic and neuromonitoring are still not routinely used. This is remarkable as both DWI-lesions and periprocedural hypotension are more common during CAS than $\mathrm{CEA}^{7,27}$. The mechanical effects of balloon angioplasty and stenting at the level of the carotid bulb are known to induce haemodynamic depression as a result of local baroreceptor stimulation and a protective effect of hypertension during CAS has been demonstrated ${ }^{28}$. More focus on haemodynamic monitoring during stenting is warranted, especially now that new type of procedures such as the TCAR are in increasingly being used. This should be considered for development of both research and clinical protocols in the near future.

\section{The effect of carotid artery disease and its treatment on cerebral perfusion}

Indication for a revascularisation procedure is currently primarily based on reduction of stroke risk by removal of the atheromatous plaque as a resource of thrombo-embolisms. However, carotid disease patients may also be at risk for cognitive decline and cerebral ischaemia due to chronic cerebral hypoperfusion ${ }^{29-31}$. As such, in addition to lowering the risk of thrombo-embolic events, optimization of cerebral haemodynamics may also be a factor to take into consideration in the process of selecting the best treatment strategy. In chapter 8 we demonstrated in a rat-model that chronic cerebral hypoperfusion, as a result of bilateral common carotid artery occlusion, leads to remarkable changes in arterial morphology as well as perivascular nerve density. Yet, these changes did not result in any significant changes in cerebral perfusion or cerebral reactivity during MRI follow-up. This suggests that, in this model, the cerebrovascular system has a large rate of adaption to compensate for such a deficiency in blood supply. From a clinical point of view this implies that severe carotid artery disease does not necessarily lead to cerebral hypoperfusion. Yet, in humans, as opposed to healthy rats, carotid artery disease is an indicator of generalized atherosclerotic disease and intracranial tandem lesions often coexists ${ }^{32}$. In addition, progressive generalized atherosclerosis may limit cerebral compensatory mechanisms ${ }^{33}$. Clinical studies are required to gain more insight on to what extend carotid artery disease and carotid revascularization establish a redistribution of blood flow. This is why we performed an ultra-high field MRI study of patients undergoing CEA in chapter 9. We aimed to investigate the effect of such a procedure on the function of the small cerebral vessels. We saw that, as a result of carotid revascularisation, increase of velocity almost always occurred in the large arteries (middle cerebral artery; MCA), while this was not necessarily accompanied with increased flow in the MCA or increased velocities in 
the perforating arteries. Vasoconstriction as a result of autoregulatory mechanisms plays an important part in this matter and the effect of revascularisation on cerebral perfusion is hard to predict. This also stresses the limitation of transcranial Doppler for assessment of changes in postprocedural cerebral perfusion, since potential alterations in vessel diameter cannot be established. We additionally demonstrated that pulsatility of the MCA decreased in the majority of patients, probably as a result of removing the atherosclerotic plaque and thus improving carotid compliance. The pulsatility of the small perforating arteries changed considerably in both directions after CEA which probably reflects the change in cerebrovascular resistance as a results of autoregulatory mechanisms ${ }^{34}$. While for most patients a revascularisation procedure may not improve cerebral perfusion, a subset of patients with insufficient collateral circulation or otherwise failing compensatory mechanisms might benefit from intervention. Future studies should continue research on this topic, specifically investigating which imaging characteristics can be preoperatively assessed to predict whether revascularisation will improve cerebral haemodynamics. When included in standard clinical workup, these features can be taken into account in decision making in asymptomatic or low-risk carotid artery disease patients. Imaging studies on cerebral small vessel function may also help to gain insight in cerebral hyperperfusion syndrome, which is nowadays still very difficult to predict with overtreatment and high hospital costs as a consequence ${ }^{35,36}$. Possibly, those that benefit most from a revascularisation procedure for improvement of cerebral perfusion are also the ones at risk for cerebral hyperperfusion syndrome. Ultra-high field MRI offers the opportunity to take a close look at the effect of proximal artery interventions on the target vessels of the brain $^{37}$. Scanning sequences and protocols alternative to the ones used in our study, such as arterial spin labelling perfusion measurements and cerebrovascular reactivity measurements may be of additional interest. These novel imaging techniques have the prospect of providing insight on cerebral haemodynamics and should be employed further in research on carotid artery disease. 


\section{Conclusion}

- In carotid artery disease, there is a strong need for a redefinition of study endpoints and a warrant for surrogate markers for ischaemic stroke, since classical outcome measures now occur at low rates.

- Patient related imaging features can be deployed to identify those at increased risk for recurrent or periprocedural brain ischaemia. Risk stratification models using these features can assist in determining optimal individualised therapeutic strategy.

- Further development of brain and carotid wall imaging protocols and subsequent implementation in standard care is essential for organising tailored therapy for patients with carotid artery disease.

- Optimisation of perioperative haemodynamic regulation in patients undergoing carotid revascularisation is critical to avert risk of cerebral complications.

- The cerebrovascular system often is capable of compensating for impeded carotid blood flow through collateral circulation and autoregulatory mechanisms. However, those in whom these mechanisms are insufficient or exhausted may have an additional benefit of carotid revascularisation for improvement of cerebral perfusion. 


\section{References}

1. Eckstein $\mathrm{H}-\mathrm{H}$, Tsantilas $\mathrm{P}$, Kühnl A, Haller B, Breitkreuz T, Zimmermann A, et al. Surgical and Endovascular Treatment of Extracranial Carotid Stenosis A Secondary Analysis of Statutory Quality Assurance Data. 2009;

2. Rots ML, van der Lugt A, de Borst GJ. Surrogate Markers and Reporting Standards for Outcome After Carotid Intervention. Eur J Vasc Endovasc Surg. 2019;7-8.

3. Lansberg MG, Norbash AM, Marks MP, Tong DC, Moseley ME, Albers GW. Advantages of adding diffusionweighted magnetic resonance imaging to conventional magnetic resonance imaging for evaluating acute stroke. Arch Neurol. 2000;57:1311-6.

4. Gensicke H, Van Der Worp HB, Nederkoorn PJ, Macdonald S, Gaines PA, Van Der Lugt A, et al. Ischemic brain lesions after carotid artery stenting increase future cerebrovascular risk. J Am Coll Cardiol. 2015;65:521-9.

5. Traenka C, Engelter ST, Brown MM, Dobson J, Frost C, Bonati LH. Silent brain infarcts on diffusionweighted imaging after carotid revascularisation: A surrogate outcome measure for procedural stroke? A systematic review and meta-analysis. Eur Stroke J. 2019;4:127-43.

6. Mrkobrada M, Chan MTV, Cowan D, Campbell D, Wang CY, Torres D, et al. Perioperative covert stroke in patients undergoing non-cardiac surgery (NeuroVISION): a prospective cohort study. Lancet. 2019;394.

7. Bonati LH, Jongen LM, Haller S, Flach HZ, Dobson J, Nederkoorn PJ, et al. New ischaemic brain lesions on MRI after stenting or endarterectomy for symptomatic carotid stenosis: a substudy of the ICSS. Lancet Neurol. 2010;9:353-62.

8. Caplan LR, Hennerici M. Impaired clearance of emboli (washout) is an important link between hypoperfusion, embolism, and ischemic stroke. Arch Neurol. 1998;55:1475-82.

9. Rots ML, Meershoek AJA, Bonati LH, den Ruijter HM, de Borst GJ. Predictors of New Ischaemic Brain Lesions on Diffusion Weighted Imaging After Carotid Stenting and Endarterectomy: A Systematic Review. Eur J Vasc Endovasc Surg. 2019;58:163-74.

10. Khanna NN, Jamthikar AD, Araki T, Gupta D, Piga M, Saba L, et al. Nonlinear model for the carotid artery disease 10-year risk prediction by fusing conventional cardiovascular factors to carotid ultrasound image phenotypes: A Japanese diabetes cohort study. Echocardiography. 2019;36:345-61.

11. Allen LM, Hasso AN, Handwerker J, Farid H. Sequence-specific MR Imaging Findings That Are Useful in Dating Ischemic Stroke. RadioGraphics. 2012;32:1285-97.

12. Meershoek AJA, de Borst GJ. Timing of carotid intervention. Br J Surg. 2018;105:1231-3.

13. Tsantilas P, Kuehnl A, König T, Breitkreuz T, Kallmayer M, Knappich C, et al. Short Time Interval Between Neurologic Event and Carotid Surgery Is Not Associated With an Increased Procedural Risk. 2016;278390.

14. Sharpe R, Sayers RD, London NJM, Bown MJ, Mccarthy MJ, Nasim A, et al. Procedural Risk Following Carotid Endarterectomy in the Hyperacute Period after Onset of Symptoms. Eur J Vasc Endovasc Surg. 2013;46:519-24.

15. Strömberg S, Gelin J, Österberg T, Bergström GML, Karlström L, Österberg K. Very urgent carotid endarterectomy confers increased procedural risk. Stroke. 2012;43:1331-5.

16. Ota H, Yarnykh VL, Ferguson MS, Underhill HR, DeMarco JK, Zhu DC, et al. Carotid Intraplaque Hemorrhage Imaging at 3.0-T MR Imaging: Comparison of the Diagnostic Performance of Three T1weighted Sequences. Radiology. 2010;254:551-63.

17. Streifler JY, Eliasziw M, Benavente OR, Alamowitch S, Fox AJ, Hachinski VC, et al. Prognostic importance of leukoaraiosis in patients with symptomatic internal carotid artery stenosis. Stroke. 2002;33:1651-5.

18. Rothwell PM, Mehta Z, Howard SC, Gutnikov SA, Warlow CP. Treating Individuals 3: From subgroups to individuals: General principles and the example of carotid endarterectomy. Lancet. 2005;365:256-65.

19. Featherstone RL, Brown MM. The second european carotid surgery trial. Endovasc today. 2012;75-7.

20. Vergouwen MDI, Backes D, van der Schaaf IC, Hendrikse J, Kleinloog R, Algra A, et al. Gadolinium Enhancement of the Aneurysm Wall in Unruptured Intracranial Aneurysms Is Associated with an Increased Risk of Aneurysm Instability: A Follow-Up Study. Am J Neuroradiol. 2019;1-5.

21. Krings T, Mandell DM, Kiehl TR, Geibprasert S, Tymianski M, Alvarez H, et al. Intracranial aneurysms: From vessel wall pathology to therapeutic approach. Nat Rev Neurol. 2011;7:547-59.

22. Portanova A, Hakakian N, Mikulis DJ, Virmani R, Abdalla WM a, Wasserman B a. Vasa vasorum 2013 insights and implications for imaging. Radiology. 2013;267:667-79.

23. Welleweerd JC, Bots ML, Kappelle LJ, Rinkel GJ, Ruigrok YM, Baas AF, et al. Rationale and design of the extracranial Carotid artery Aneurysm Registry (CAR). J Cardiovasc Surg (Torino). 2018;59:692-8.

24. van Klei WA, van Waes JAR, Pasma W, Kappen TH, van Wolfswinkel L, Peelen LM, et al. Relationship Between Preoperative Evaluation Blood Pressure and Preinduction Blood Pressure. Anesth Analg. 2017;124:431-7. 
25. Verberk WJ, Kroon AA, Thien T, Lenders JWM, Van Montfrans GA, Smit AJ, et al. Prevalence of the white-coat effect at multiple visits before and during treatment. J Hypertens. 2006;24:2357-63.

26. Bond R, Narayan SK, Rothwell PM, Warlow CP. Clinical and radiographic risk factors for operative stroke and death in the European Carotid Surgery Trial. Eur J Vasc Endovasc Surg. 2002;23:108-16.

27. Lavoie P, Rutledge J, Dawoud MA, Mazumdar M, Riina H, Gobin YP. Predictors and timing of hypotension and bradycardia after carotid artery stenting. Am J Neuroradiol. 2008;29:1942-7.

28. Nanto M, Goto Y, Yamamoto H, Tanigawa S, Takeuchi H, Nakahara Y, et al. Complications and predictors of hypotension requiring vasopressor after carotid artery stenting. Neurol Med Chir (Tokyo). 2017;57:115-21.

29. Duncombe J, Kitamura A, Hase Y, Ihara M, Kalaria R, Horsburgh K. Chronic cerebral hypoperfusion: a key mechanism leading to vascular cognitive impairment and dementia (VCID). Closing the translational gap between rodent models and human VCID. Clin Sci. 2017;131:2451-68.

30. Göttler J, Kaczmarz S, Nuttall R, Griese V, Zimmer C, Sorg C, et al. One-sided hypoperfusion is associated with contralateral attention deficits in asymptomatic high-grade carotidstenosis patients. J Cereb Blood Flow Metab. 2017;37:499-500.

31. Klijn CJM, Kappelle LJ, Tulleken CAF, van Gijn JM. Symptomatic Carotid Artery Occlusion: A Reappraisal of Hemodynamic Factors. Stroke. 1997;28:2084-2093.

32. Kappelle LJ, Eliasziw M, Fox AJ, Sharpe BL, Barnett HJ. Importance of intracranial atherosclerotic disease in patients with symptomatic stenosis of the internal carotid artery. The North American Symptomatic Carotid Endarterectomy Trail. Stroke. 1999;30:282-6.

33. Doux JD, Yun AJ. The link between carotid artery disease and ischemic stroke may be partially attributable to autonomic dysfunction and failure of cerebrovascular autoregulation triggered by Darwinian maladaptation of the carotid baroreceptors and chemoreceptors. Med Hypotheses. 2006;66:176-81.

34. Duffin J, Sobczyk O, McKetton L, Crawley A, Poublanc J, Venkatraghavan L, et al. Cerebrovascular resistance: The basis of cerebrovascular reactivity. Front Neurosci. 2018;12:1-14.

35. Dalman JE, Beenakkers ICM, Moll FL, Leusink JA, R.G.A. Ackerstaff. Transcranial Doppler monitoring during carotid endarterectomy helps to identify patients at risk of postoperative hyperperfusion. Eur J Vasc Endovasc Surg. 1999;18:222-7.

36. Fassaert LMM, Immink R V., van Vriesland DJ, de Vries J-PPM, Toorop RJ, Kappelle LJ, et al. Transcranial Doppler 24 Hours after Carotid Endarterectomy Accurately Identifies Patients Not at Risk of Cerebral Hyperperfusion Syndrome. Eur J Vasc Endovasc Surg. 2019;58:320-7.

37. Geurts LJ, Zwanenburg JJM, Klijn CJM, Luijten PR, Biessels GJ. Higher Pulsatility in Cerebral Perforating Arteries in Patients With Small Vessel Disease Related Stroke, a 7T MRI Study. Stroke. 2019;50:62-8. 
Discussion, future perspectives and conclusion 


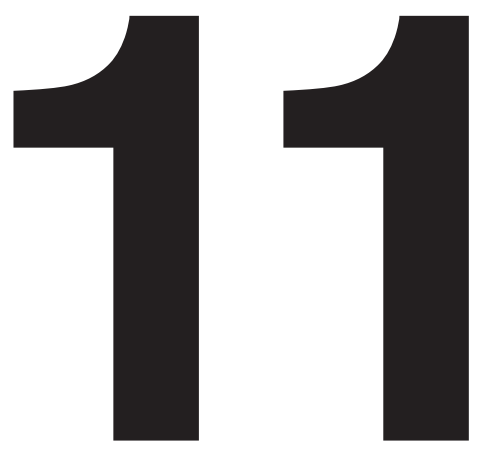

Nederlandse samenvatting

(summary in Dutch) 


\section{Nederlandse samenvatting}

In dit proefschrift worden verscheidene aspecten beschreven voor het optimaliseren van zorg voor mensen met een vernauwing van de halsslagader (stenose van de arteria carotis). We richten ons hierbij met name op de vraag hoe een betere patiëntselectie kan plaatsvinden, door op individueel niveau een inschatting te maken van zowel de potentiele voordelen van een behandeling alsook de risico's die hiermee gepaard gaan.

Een stenose van de a. carotis wordt meestal veroorzaakt door atherosclerose, een vorm van aderverkalking, waarbij een atherosclerotische plaque het lumen van het vat vernauwt en hiermee interfereert met de doorbloeding in dit vat. Patiënten met een dergelijke stenose hebben een verhoogd risico op het krijgen van een cerebrovasculair accident (CVA); in tien tot vijftien procent van alle (ischemische) CVA's kan de oorzaak hiervoor gevonden worden in de a. carotis. In het merendeel van de gevallen komt dit risico voort uit het feit dat een instabiele atherosclerotische plaque in de vaatwand scheurt (ruptureert), waarbij embolieën loskomen die een verstopping (occlusie) veroorzaken in kleinere, meer distaal gelegen intracraniële vaten. Andere, meer zeldzame, aandoeningen van de a. carotis zoals een extracranieel carotis aneurysma kunnen ook dergelijke embolieën veroorzaken volgens een zelfde mechanisme. Een ander potentieel risico dat mensen met een stenose van de a. carotis lopen is een verminderde doorbloeding van de hersenen omdat de stenose dusdanig vergevorderd is dat er nauwelijks meer bloed passeert. Onder normale omstandigheden zou het lichaam een dergelijk gebrek aan doorbloeding vanuit een van de carotiden moeten kunnen compenseren, maar dit kan tekortschieten als er sprake is van een niet-competente collaterale circulatie of aangedane vaten op een ander niveau. Chronische verminderde perfusie van het brein kan leiden tot cognitieve achteruitgang en daarnaast kan dit ook een risico geven op hersenschade door zuurstofkort (ischemie) in de kwetsbare waterscheidingsgebieden van het brein.

Het behandelen van de a. carotis door een chirurgische verwijdering van de plaque (carotid endarterectomy, CEA) of een endovasculaire stentplaatsing (carotid artery stenting, CAS) is effectief gebleken in het voorkomen van een recidief CVA in patiënten met eerdere symptomen. Echter lijken voor patiënten met een a. carotis stenose zonder eerdere symptomen ('asymptomaten') de risico's die gepaard gaan met de ingreep niet op te wegen tegen de voordelen van zo'n procedure. Door verbeteringen in medicamenteuze behandeling en levensstijl adviezen is het risico op (recidief) CVA in de onbehandelde patiënt gedaald, waar tegelijkertijd deze betere medicatie in combinatie met medisch-technische ontwikkelingen ook het peri-procedurele risico ook heeft doen dalen. De consequentie van deze positieve ontwikkeling is dat voor onderzoek naar de meerwaarde van een bepaalde interventie grote aantallen patiënten benodigd zijn om een potentieel effect te kunnen meten op klassieke uitkomstmaten zoals een CVA of cardiovasculaire dood. Om die reden is er een grote vraag naar een betrouwbare vervangende (surrogaat) uitkomst marker die ingezet kan worden om peri-procedurele en lange termijn risico's te kunnen bepalen. Daarnaast kan meer inzicht in bijkomende potentiele voordelen van een ingreep, zoals verbetering van de doorbloeding van het brein, bijdragen aan de besluitvorming bij de patiënt waar twijfel bestaat of het voordeel van een ingreep opweegt tegen de risico's hiervan. 
In hoofdstuk 2 bespreken we de best bekende surrogaatmaten voor uitkomst bij patiënten met een a. carotis stenose en bepleiten we dat deze radiologische markers geïmplementeerd zouden moeten worden in de dagelijkse klinische praktijk. Diffusion weighted imaging (DWI)-laesies zijn markers op MRI die in een zeer vroeg stadium (tijdelijk) zuurstoftekort in het brein reflecteren, zonder dat dit gepaard hoeft te gaan met symptomen. DWI-laesies worden in toenemende mate ingezet als veelbelovende surrogaat uitkomstmarker voor periprocedureel CVA. Witte stof afwijkingen en stille infarcten zijn surrogaat uitkomstmaten op MRI of CT en zijn beter geschikt voor het meten van lange termijn uitkomst na carotis interventie of conservatieve behandeling. Standaardisatie van beeldvorming protocollen en methode van rapporteren is cruciaal om meer kennis te vergaren over bruikbaarheid en betrouwbaarheid van deze markers. Deze DWI-laesies gebruiken we in de systematische review in hoofdstuk 3 om patiëntkarakteristieken te identificeren die geassocieerd zijn met een verhoogd risico op slechtere uitkomst rondom een ingreep van de carotis middels CEA of CAS. De in de literatuur onderzochte patiënt karakteristieken bleken voor beide behandelingen zeer heterogeen en onderling verschillend. We vonden dat verminderde cerebrale perfusie, verhoogde ontstekingswaarden en symptomatisch zijn een verhoogd risico gaven op nieuwe DWI-laesies na CEA terwijl een hogere leeftijd, plaque instabiliteit en complexe anatomie van het vaatstelsel een verhoogd risico gaven na CAS. Deze informatie kan helpen voor het maken van een risicoprofiel en daarmee assisteren in de keuze voor een van de twee behandelingen. In hoofdstuk 4 bespreken we of patiënt-gerelateerde factoren kunnen bijdragen aan de besluitvorming rondom de behandelstrategie als we gebruik maken van plaque eigenschappen voor de risicoprofilering. We vonden dat patiënten met tekenen van een instabiele carotis plaque een verhoogd risico hebben op recidief ischemie in de wachtperiode tussen eerste symptoom en CEA. Dit suggereert dat het vooraf identificeren van mensen met tekenen van plaque instabiliteit zou kunnen helpen in het prioriteren van zorg. Deze informatie kan bijdragen aan de discussie of behandeling vroeg ( $<14$ dagen) of versneld ( $<48$ uur) na de eerste symptomen moet plaatsvinden. Ook wanneer de wachttijd $>14$ dagen was vonden we dat een instabiele plaque een risico geeft op recidief ischemie, wat suggereert dat een snelle behandeling ook geïndiceerd is wanneer er een vertraging in ziekenhuis presentatie heeft plaatsgevonden.

In hoofdstuk 5 onderzoeken we of standaard preoperatieve beeldvorming van de hersenen middels CT of MRI kan helpen in het voorspellen van korte of lange termijn uitkomst na CEA. We vonden dat aanwezigheid van witte stof afwijkingen voorspellend is voor lange termijn cardiovasculaire dood, maar niet voor peri-procedureel of lange termijn risico op een CVA. Deze informatie kan bijdragen in de afwegingen voor het wel/niet uitvoeren van een ingreep omdat wellicht het aantal jaren profijt van een ingreep minder is bij mensen met witte stof afwijkingen. Toekomstige risicomodellen die helpen bij het kiezen tussen conservatieve of invasieve (CEA/CAS) behandeling kunnen baat hebben bij het betrekken van aanwezigheid van preoperatieve witte stof afwijkingen. In hoofdstuk 6 beschrijven we een studie waarin we hebben onderzocht of beeldvorming bij mensen met een extracranieel carotis aneurysma kan helpen in het voorspellen van risico op complicaties. Een conservatieve behandeling van zo'n aneurysma lijkt meestal gerechtvaardigd maar sommige patiënten zullen wel baat 
hebben bij een invasieve procedure ter voorkoming van cerebrale complicaties. Het vooraf identificeren van die patiënten is uitdagend, ook omdat er nog weinig bekend is over deze zeldzame aandoening. Aankleuring van de wand van het aneurysma op MRI met contrast kan mogelijk een aanwijzing zijn voor instabiliteit en daarmee groei van het aneurysma en mogelijke embolieën naar het brein. In deze studie hebben we voor het eerst aangetoond dat afbeelden van de vaatwand van een carotis aneurysma mogelijk is en dat de beoordeling hiervan met goede reproduceerbaarheid kan plaatsvinden. Toekomstig onderzoek moet verder duidelijk maken of dit type beeldvorming ingezet kan worden om mensen te identificeren die baat hebben bij een behandeling van het carotis aneurysma.

Een invasieve ingreep aan de a. carotis (CEA/CAS) is niet zonder risico's. Het is van belang dat tijdens de procedure de doorbloeding van het brein optimaal blijft, om de kans op zuurstoftekort te minimaliseren. Daarom onderzoeken we in hoofdstuk 7 in hoeverre intra-operatieve bloeddruk van invloed is op het risico op post-operatieve DWI-laesies. We vonden dat met name mensen met een preoperatief hoge bloeddruk een hoog risico hadden op een relatief lage bloeddruk tijdens de procedure, wat een verhoogd risico gaf op nieuwe DWI-laesies. Het optimaliseren van het bloeddruk beleid rondom de behandeling lijkt essentieel voor het verlagen van de kans op complicaties tijdens CEA of CAS. Mogelijk is bij deze mensen de autoregulatie dusdanig uitgeput dat het compenseren voor (tijdelijke) bloeddrukdalingen tijdens de ingreep onvoldoende mogelijk is.

In hoofdstuk $\mathbf{8}$ beschrijven we met behulp van een rat-model wat het effect is van chronisch verminderde doorbloeding van het brein op de bloedvaten die het brein van bloed voorzien. We demonstreren dat er opvallende veranderingen plaatsvinden in dit cerebrovasculaire systeem om te compenseren voor het gebrek aan bloedtoevoer vanuit de carotiden. Hiermee werd opvallend genoeg de totale doorbloeding van het brein op peil gehouden. We laten hiermee zijn dat het brein goed in staat is om door compensatoire mechanismes (autoregulatie en collaterale bloedflow) te corrigeren voor verminderde doorbloeding van de carotiden. Een eventuele ingreep aan de a. carotis hoeft dus niet noodzakelijkerwijs ook een verbetering van de doorbloedig van het brein te geven. Daarom proberen we in hoofdstuk 9 te achterhalen wat het effect is van een operatie aan de a. carotis op de doorbloeding van bloedvaten in het brein. We zien daarbij dat er een verandering plaatsvindt in de doorbloeding van de grote bloedvaten maar dat dit niet noodzakelijkerwijs gepaard gaat met een zelfde verandering in de klein bloedvaten. In overeenstemming met het vorige hoofdstuk bevestigt dit dat het vaatstelsel in het brein heel goed in staat is om te compenseren voor tekorten en veranderingen in de bloedtoevoer. In gevallen waarbij dit compensatiemechanisme niet adequaat of uitgeput is, is er een duidelijk effect te zien van een ingreep aan de a. carotis op de doorbloeding van de kleine vaatjes in het brein. De uitdaging voor de toekomst is om deze mensen op voorhand te kunnen identificeren zodat dit mee kan wegen in de besluitvorming omtrent het kiezen voor een invasieve of conservatieve behandelstrategie. 
Nederlandse samenvatting 


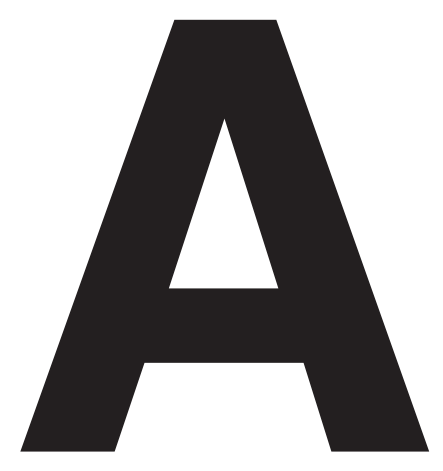

\section{Appendices}

Review committee

Authors and Affiliations

List of publications

Acknowledgements | Dankwoord

About the author 
Appendices 


\section{Review committee}

Prof. Dr. R.M. Dijkhuizen

Prof. Dr. L.J. Kappelle

Prof. Dr. G. Pasterkamp

Prof. Dr. A. van der Lugt

Prof. Dr. L.H. Bonati 


\section{Authors and Affiliation}

Tine Arts, MSc.

Department of Radiology

University Medical Center Utrecht

Utrecht, the Netherlands

Leo. H. Bonati, MD, PhD

Department of Neurology and Stroke Center

University Hospital Basel

Basel, Switzerland

Gert J. de Borst, MD, PhD

Department of Vascular Surgery

University Medical Center Utrecht

Utrecht, the Netherlands

Martin M. Brown. MD, PhD

Department of Neurology and Neurosurgery

University College London Hospitals

London, United Kingdom

Rick M. Dijkhuizen, MD, PhD

Center for Image Sciences

University Medical Center Utrecht

Utrecht, the Netherlands

Leonie M. Fassaert, MD, PhD

Department of Vascular Surgery

University Medical Center Utrecht

Utrecht, the Netherlands

Mark C.H. de Groot, PhD

Departments of Clinical Chemistry and Haematology

University Medical Center Utrecht

Utrecht, the Netherlands
Saskia Haitjema, MD, PhD

Departments of Clinical Chemistry and

Haematology

University Medical Center Utrecht

Utrecht, the Netherlands

Jeroen Hendrikse, MD, PhD

Department of Radiology

University Medical Center Utrecht

Utrecht, the Netherlands

Nikita K.N. Jorritsma, BSc.

Department of Vascular Surgery

University Medical Center Utrecht

Utrecht, the Netherlands

L. Jaap Kappelle, MD, PhD

Department of Neurology and Neurosurgery

University Medical Center Utrecht

Utrecht, the Netherlands

Wilton. A. van Klei, MD, PhD

Department of Anaesthesiology

University Medical Center Utrecht

Utrecht, the Netherlands

Dominique P.V. de Kleijn, PhD

Department of Vascular Surgery

University Medical Center Utrecht

Utrecht, the Netherlands

Ian D. van Koeverden, MD, PhD

Department of Vascular Surgery

University Medical Center Utrecht

Utrecht, the Netherlands 
Constance J.H.C.M. van Laarhoven, MD Department of Vascular Surgery University Medical Center Utrecht Utrecht, the Netherlands

Tim Leiner, MD, PhD

Department of Radiology

University Medical Center Utrecht

Utrecht, the Netherlands

Aad van der Lugt, MD, PhD

Department of Radiology \& Nuclear Medicine

Erasmus Medical Center

Rotterdam, the Netherlands

Armelle J.A. Meershoek, MD, PhD

Department of Vascular Surgery

University Medical Center Utrecht

Utrecht, the Netherlands

Frans L. Moll, MD, PhD

Department of Vascular Surgery

University Medical Center Utrecht

Utrecht, the Netherlands

Gerard Pasterkamp, MD, PhD

Experimental Cardiology Laboratory

University Medical Center Utrecht

Utrecht, the Netherlands

Claire W.A. Pennekamp, MD, PhD

Department of Vascular Surgery

University Medical Center Utrecht

Utrecht, the Netherlands
Vanessa E.C. Pourier, MD, PhD

Department of Vascular Surgery

University Medical Center Utrecht

Utrecht, the Netherlands

Hester M. den Ruijter, PhD

Experimental Cardiology Laboratory

University Medical Center Utrecht

Utrecht, the Netherlands

Nathalie Timmerman, MD

Department of Vascular Surgery

University Medical Center Utrecht

Utrecht, the Netherlands

Annette van der Toorn, PhD

Center for Image Sciences

University Medical Center Utrecht

Utrecht, the Netherlands

Mervyn D.I. Vergouwen, MD, PhD

Department of Neurology and Neurosurgery

University Medical Center Utrecht

Utrecht, the Netherlands

Jaco J.M. Zwanenburg, PhD

Department of Radiology

University Medical Center Utrecht

Utrecht, the Netherlands 


\section{List of publications}

Timmerman N, Rots ML, van Koeverden ID, Haitjema S, van Laarhoven CJHCM, Vuurens AM, den Ruijter HM, Pasterkamp G, Kappelle LJ, de Kleijn DPV, de Borst GJ, Cerebral small vessel disease in standard preoperative imaging reports is independently associated with increased risk of cardiovascular death following carotid endarterectomy, Eur J Vasc Endovasc Surg, 2020; in press

Van Laarhoven CJHCM, Rots ML, Pourier VEC, Jorritsma NKN, Leiner T, Hendrikse $\mathrm{J}$, Vergouwen MDI, de Borst GJ, Gadolium-enhancement of the aneurysm wall in extracranial carotid artery aneurysms, Am J Neuroradiol. 2020; in press

Rots ML, Fassaert LMM, Kappelle LJ, de Groot MCH, Haitjema S, Bonati LH, van Klei WA, de Borst GJ, Intraoperative hypotension is a risk factor for postoperative silent brain ischaemia in patients with preoperative hypertension undergoing carotid endarterectomy. Eur J Vasc Endovasc Surg. 2020; in press

Rots ML, de Borst GJ. Commentary on "Overview of Primary and Secondary Analyses From 20 Randomised Controlled Trials Comparing Carotid Artery Stenting With Carotid Endarterectomy. Eur J Vasc Endovasc Surg. 2019; in press

Rots ML, van der Lugt A, de Borst GJ. Surrogate Markers and Reporting Standards for Outcome after Carotid Intervention. Eur J Vasc Endovasc Surg 2019;58(6):794-795

Rots ML, Timmerman N, de Kleijn DPV, Pasterkamp G, Brown MM, Bonati LH, de Borst GJ. Magnetic resonance imaging identified brain ischaemia in symptomatic patients undergoing carotid endarterectomy is related to histologically apparent intraplaque haemorrhage. Eur J Vasc Endovasc Surg 2019;58(6):796-804

Rots ML, Meershoek AJA, Bonati LH, den Ruijter HM, de Borst GJ. Predictors of New Ischaemic Brain Lesions on Diffusion Weighted Imaging After Carotid Stenting and Endarterectomy: A Systematic Review. Eur J Vasc Endovasc Surg. 2019;58(2):163-74.

Rots ML, de Borst GJ, van der Toorn A, Moll FL, Pennekamp CWA, Dijkhuizen RM, et al. Effect of bilateral carotid occlusion on cerebral hemodynamics and perivascular innervation: An experimental rat model. J Comp Neurol. 2019;527(14):2263-2272.

Poodt IGM, Rots ML, Vugts G, van Dalen T, Kuijer A, Vriens BEPJ, et al. The administration of adjuvant chemo(-immuno) therapy in the post ACOSOG-Z0011 era; a population based study. Eur J Surg Oncol. 2018;44(8):1151-6.

Poodt IGM, Spronk PER, Vugts G, van Dalen T, Vrancken Peeters MTFD, Rots ML, et al. Trends on axillary surgery in nondistant metastatic breast cancer patients treated between 2011 and 2015: A dutch population-based study in the ACOSOG-Z0011 and AMAROS era. Ann Surg. 2017;268(6):1084-90.

Rots ML, van Putten MJAM, Hoedemaekers CWE, Horn J. Continuous EEG Monitoring for Early Detection of Delayed Cerebral Ischemia in Subarachnoid Hemorrhage: A Pilot Study. Neurocrit Care. 2016;24(2):207-16. 
Van Der Kooi AW, Rots ML, Huiskamp G, Klijn FAM, Koek HL, Kluin J, et al. Delirium detection based on monitoring of blinks and eye movements. Am J Geriatr Psychiatry. 2014;22(12):1575-82.

Rots ML, De Vos CC, Smeets-Schouten JS, Portier R, van Putten MJAM. Suppressors of interictal discharges in idiopathic childhood occipital epilepsy of Gastaut. Epilepsy Behav. 2012;25(2):189-91. 
Appendices 


\section{Acknowledgements I Dankwoord}

Mensen die zeggen dat onderzoek doen eenzaam is hebben ongelijk; dit proefschrift was nooit tot stand gekomen zonder alle steun en aanmoediging van zo veel fantastische mensen. Of het nou ging om inhoudelijk advies, inspirerende gesprekken, het uitspreken van vertrouwen of een klein momentje van verzet, ze hebben allemaal bijgedragen aan het voltooien van mijn promotieonderzoek.

Ik wil in het bijzonder mijn (co-)promotoren bedanken. Jaco, wat was het goed om tijdens mijn promotietraject iemand te hebben die verstand had van alles waar ik weinig van begreep. Door jouw scherpe en kritische blik heb je mij, als (soms iets te) pragmatische, chirurgisch georiënteerde onderzoeker een andere invalshoek laten zien en hebben we een mooie basis gelegd voor klinisch carotis onderzoek met inzet van nieuwe imaging technieken. Jeroen, onze meetings waren sporadisch, en af en toe slechts en passant in de gang, maar daardoor zeker niet minder zinvol. Het was altijd motiverend om te merken met hoeveel positiviteit je reageerde op de lopende projecten en jouw visie te horen op de vervolgstappen van dit onderzoek. Gert Jan, jouw eindeloze hoeveelheid enthousiasme en steun was inspirerend. Ik sta nog steeds versteld van het geduld en de oprechtheid waarmee je mij en alle andere promovendi onder je hoede hebt genomen, ondanks dat we met zo velen zijn. Door ambitie, humor en zo nodig een kritische noot op meetings, congressen en in eindeloos veel mails (waar ik nooit langer dan 24u op hoefde te wachten) zorgde je ervoor dat ik altijd gemotiveerd was om door te gaan en nieuwe projecten op te pakken, met alle vrijheid om hier zelf keuzes in te maken. Ik keek zonder uitzondering met veel plezier uit naar onze cappuccino-overlegmomenten omdat ik wist dat daarna de stip op de horizon weer scherp in het vizier kwam. Veel dank voor deze mooie jaren!

Lieve (oud-)collega's, vrienden, familie, paranimfen en Zwier dank jullie wel voor alle support, inspiratie, afleiding, slechte grappen en geduld, het zou niet gelukt zijn zonder jullie en het zou bovenal lang niet zo leuk zijn geweest.

Gert Jan de Borst I Jeroen Hendrikse I Jaco Zwanenburg I Leescommissie I Co-auteurs I Vaatchirurgen UMC Utrecht I Dominique de Kleijn I Cobie van Veen I Susan Hora Siccama I Menno Vriens I Thijs van Dalen I Annelinde Veen I Tesse Leunissen I Robert de Kleijn I Armelle Meershoek I Aarent Brand I Bernard Leenstra I Joep Wijnand I Nicolaas Roozendaal I Maarten Verwer I Janine Simons I Leonie Fassaert I Constance van Laarhoven I Marieke Walma I Dirk-Jan van Beek I Bianca den Dekker I Lutske Lodewijk I De $7^{\mathrm{e}}$ | De Toren I Vaatonderzoekers UMC Utrecht I SkiCie '19 I Alle (oud-)onderzoekers, assistenten \& stafleden van het UMC Utrecht I Oud-collega's Diakonessenhuis I Vrienden \& Vriendinnen Mary \& Ino I Familie \& Schoonfamilie Zwier 
Appendices 


\section{About the author}

Marjolijn Lette Rots was born on September 17, 1988 in Naarden, the Netherlands. She is the daughter of Ino Rots and Mary Dankbaar and grew up in Hilversum. After graduating from the A. Roland Holst College in Hilversum in 2006 she moved to Enschede to study Technical Medicine. During her studies, she gained interest in surgical disciplines and critical care medicine and decided to focus on these topics during her master, in the course of which she did four clinical internships in several Dutch hospitals and one internship at the department of biomedical engineering at the University of Florida. During these internships her ambition to work with patients was triggered and she decided to study Medicine at the University of Utrecht after getting her master's degree in Technical Medicine in 2013.

Throughout her medical training, Marjolijn's interest in the surgical field was fortified. After graduating in 2016, she started to work as a surgical resident (not in training) at the Diakonessenhuis in Utrecht, where she worked for one and a half year. While working as a resident she was introduced to prof. dr. G.J. de Borst and they discussed possibilities for research on deployment of novel imaging methods in patients with carotid artery disease, in which technical innovations and clinical research could be joined. Professor G.J. de Borst, professor and J. Hendrikse and dr. J.J.M. Zwanenburg gave her the opportunity to start on this project combining vascular surgery and radiology as a $\mathrm{PhD}$-candidate at the University Medical Center in Utrecht, the results of which are presented in this thesis. In 2020 Marjolijn will start with her surgical residency (in training) at the Jeroen Bosch Ziekenhuis in Den Bosch under supervision of dr. W. Brokelman and dr. K. Bosscha. Marjolijn lives in Utrecht with Zwier Smith and enjoys sports, traveling and spending time with friends and family. 



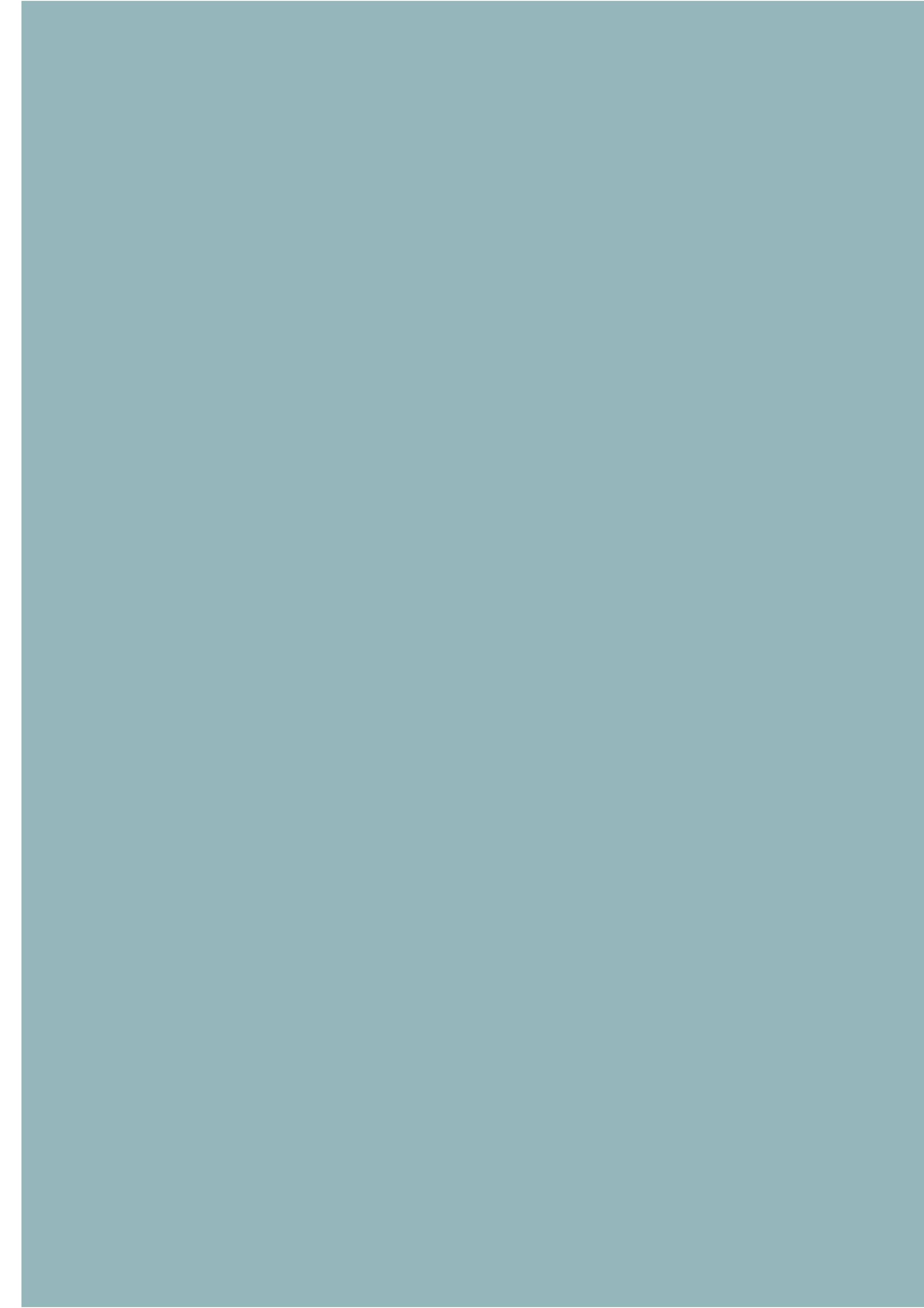


